\title{
Oral hygiene interventions for people with intellectual disabilities
} (Review)

Waldron C, Nunn J, Mac Giolla Phadraig C, Comiskey C, Guerin S, van Harten MT, Donnelly-Swift E, Clarke MJ 
TABLE OF CONTENTS

ABSTRACT

PLAIN LANGUAGE SUMMARY

SUMMARY OF FINDINGS

BACKGROUND

OBJECTIVES

METHODS

Figure 1.

Figure 2.

RESULTS

Figure 3.

DISCUSSION

AUTHORS' CONCLUSIONS

ACKNOWLEDGEMENTS

REFERENCES

CHARACTERISTICS OF STUDIES

DATA AND ANALYSES

Analysis 1.1. Comparison 1 Special manual toothbrush (TB) versus conventional manual TB (used by carers) for people with intellectual disabilities (ID), Outcome 1 Gingival inflammation short term (<6 weeks).

Analysis 1.2. Comparison 1 Special manual toothbrush (TB) versus conventional manual TB (used by carers) for people with intellectual disabilities (ID), Outcome 2 Gingival inflammation medium term (6 weeks to 12 months).

Analysis 1.3. Comparison 1 Special manual toothbrush (TB) versus conventional manual TB (used by carers) for people with intellectual disabilities (ID), Outcome 3 Plaque short term $(<6$ weeks).

Analysis 1.4. Comparison 1 Special manual toothbrush (TB) versus conventional manual TB (used by carers) for people with intellectual disabilities (ID), Outcome 4 Plaque medium term (6 weeks to 12 months).

Analysis 1.5. Comparison 1 Special manual toothbrush (TB) versus conventional manual TB (used by carers) for people with intellectual disabilities (ID), Outcome 5 Oral health assessments.

Analysis 1.6. Comparison 1 Special manual toothbrush (TB) versus conventional manual TB (used by carers) for people with intellectual disabilities (ID), Outcome 6 Behaviour, attitude and self-efficacy.

Analysis 1.7. Comparison 1 Special manual toothbrush (TB) versus conventional manual TB (used by carers) for people with intellectual disabilities (ID), Outcome 7 Degree of resistance of person with ID.

Analysis 2.1. Comparison 2 Electric toothbrush (TB) versus manual TB for people with intellectual disabilities, Outcome 1 Gingival inflammation medium term ( 6 weeks to 12 months).

Analysis 2.2. Comparison 2 Electric toothbrush (TB) versus manual TB for people with intellectual disabilities, Outcome 2 Plaque medium term (6 weeks to 12 months).

Analysis 2.3. Comparison 2 Electric toothbrush (TB) versus manual TB for people with intellectual disabilities, Outcome 3 NRS Gingival inflammation long term (> 12 months).

Analysis 2.4. Comparison 2 Electric toothbrush (TB) versus manual TB for people with intellectual disabilities, Outcome 4 NRS Plaque medium term ( 6 weeks to 12 months).

Analysis 2.5. Comparison 2 Electric toothbrush (TB) versus manual TB for people with intellectual disabilities, Outcome 5 Calculus medium term ( 6 weeks to 12 months).

Analysis 3.1. Comparison 3 Training of carers of people with intellectual disabilities versus no training of carers, Outcome 1 Gingival inflammation medium term (6 weeks to 12 months).

Analysis 3.2. Comparison 3 Training of carers of people with intellectual disabilities versus no training of carers, Outcome 2 Plaque medium term ( 6 weeks to 12 months).

Analysis 3.3. Comparison 3 Training of carers of people with intellectual disabilities versus no training of carers, Outcome 3 Non-randomised study plaque short term ( $<6$ weeks).

Analysis 3.4. Comparison 3 Training of carers of people with intellectual disabilities versus no training of carers, Outcome 4 Knowledge short term ( $<6$ weeks).

Analysis 3.5. Comparison 3 Training of carers of people with intellectual disabilities versus no training of carers, Outcome 5 Knowledge medium term (6 weeks to 12 months).

Analysis 3.6. Comparison 3 Training of carers of people with intellectual disabilities versus no training of carers, Outcome 6 Behaviour, attitude and self-efficacy medium term.

Analysis 4.1. Comparison 4 Oral health $(\mathrm{OH})$ training of people with intellectual disabilities (ID) versus no training, Outcome 1 Gingival inflammation short term ( $<6$ weeks). 
Analysis 4.2. Comparison 4 Oral health $(\mathrm{OH})$ training of people with intellectual disabilities (ID) versus no training, Outcome 2 Plaque short term (<6 weeks).

Analysis 5.1. Comparison 5 Dental recall intervals for people with intellectual disabilities, Outcome 1 Gingival pocketing long term (> 12 months).

Analysis 5.2. Comparison 5 Dental recall intervals for people with intellectual disabilities, Outcome 2 Gingival bleeding long term (> 12 months).

Analysis 5.3. Comparison 5 Dental recall intervals for people with intellectual disabilities, Outcome 3 Plaque long term (> 12 months).

Analysis 5.4. Comparison 5 Dental recall intervals for people with intellectual disabilities, Outcome 4 Calculus long term (> 12 months).

Analysis 6.1. Comparison 6 Clinical photographs as motivators for oral hygiene in people with intellectual disabilities, Outcome 1 Plaque medium term (6 weeks to 12 months).

ADDITIONAL TABLES 
[Intervention Review]

\section{Oral hygiene interventions for people with intellectual disabilities}

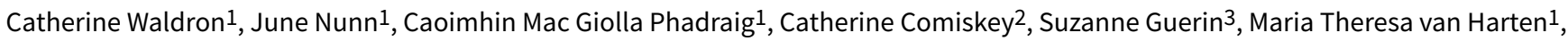
Erica Donnelly-Swift ${ }^{1}$, Mike J Clarke 4

1School of Dental Science, Trinity College Dublin, University of Dublin, Dublin, Ireland. 2School of Nursing and Midwifery, Trinity College Dublin, University of Dublin, Dublin, Ireland. ${ }^{3}$ School of Psychology, University College Dublin, Dublin, Ireland. ${ }^{4}$ Centre for Public Health, Queen's University Belfast, Belfast, UK

Contact: Catherine Waldron, School of Dental Science, Trinity College Dublin, University of Dublin, Lincoln Place, Dublin, D02 F859, Ireland.waldrocg@tcd.ie,waldrocg@tcd.ie.

Editorial group: Cochrane Oral Health Group.

Publication status and date: Edited (no change to conclusions), published in Issue 6, 2019.

Citation: Waldron C, Nunn J, Mac Giolla Phadraig C, Comiskey C, Guerin S, van Harten MT, Donnelly-Swift E, Clarke MJ. Oral hygiene interventions for people with intellectual disabilities. Cochrane Database of Systematic Reviews 2019, Issue 5. Art. No.: CD012628. DOI: 10.1002/14651858.CD012628.pub2.

Copyright @ 2019 The Cochrane Collaboration. Published by John Wiley \& Sons, Ltd.

\section{A B S T R A C T}

\section{Background}

Periodontal (gum) disease and dental caries (tooth decay) are the most common causes of tooth loss; dental plaque plays a major role in the development of these diseases. Effective oral hygiene involves removing dental plaque, for example, by regular toothbrushing. People with intellectual disabilities (ID) can have poor oral hygiene and oral health outcomes.

\section{Objectives}

To assess the effects (benefits and harms) of oral hygiene interventions, specifically the mechanical removal of plaque, for people with intellectual disabilities (ID).

\section{Search methods}

Cochrane Oral Health's Information Specialist searched the following databases to 4 February 2019: Cochrane Oral Health's Trials Register, the Cochrane Central Register of Controlled Trials (CENTRAL; Cochrane Register of Studies), MEDLINE Ovid, Embase Ovid and PsycINFO Ovid. ClinicalTrials.gov and the World Health Organization International Clinical Trials Registry Platform were searched for ongoing trials. The Embase search was restricted by date due to the Cochrane Centralised Search Project, which makes available clinical trials indexed in Embase through CENTRAL. We handsearched specialist conference abstracts from the International Association of Disability and Oral Health (2006 to 2016).

\section{Selection criteria}

We included randomised controlled trials (RCTs) and some types of non-randomised studies (NRS) (non-RCTs, controlled before-after studies, interrupted time series studies and repeated measures studies) that evaluated oral hygiene interventions targeted at people with ID or their carers, or both. We used the definition of ID in the International Statistical Classification of Diseases and Related Health Problems, 10th revision (ICD-10). We defined oral hygiene as the mechanical removal of plaque. We excluded studies that evaluated chemical removal of plaque, or mechanical and chemical removal of plaque combined.

\section{Data collection and analysis}

At least two review authors independently screened search records, identified relevant studies, extracted data, assessed risk of bias and judged the certainty of the evidence according to GRADE criteria. We contacted study authors for additional information if required. We reported RCTs and NRSs separately. 


\section{Main results}

We included 19 RCTs and 15 NRSs involving 1795 adults and children with ID and 354 carers. Interventions evaluated were: special manual toothbrushes, electric toothbrushes, oral hygiene training, scheduled dental visits plus supervised toothbrushing, discussion of clinical photographs showing plaque, varied frequency of toothbrushing, plaque-disclosing agents and individualised care plans. We categorised results as short (six weeks or less), medium (between six weeks and 12 months) and long term (more than 12 months).

Most studies were small; all were at overall high or unclear risk of bias. None of the studies reported quality of life or dental caries. We present below the evidence available from RCTs (or NRS if the comparison had no RCTs) for gingival health (inflammation and plaque) and adverse effects, as well as knowledge and behaviour outcomes for the training studies.

Very low-certainty evidence suggested a special manual toothbrush (the Superbrush) reduced gingival inflammation (GI), and possibly plaque, more than a conventional toothbrush in the medium term ( $\mathrm{Gl}$ : mean difference (MD) $-12.40,95 \% \mathrm{Cl}-24.31$ to -0.49 ; plaque: $\mathrm{MD}$ $-0.44,95 \% \mathrm{Cl}-0.93$ to $0.05 ; 1 \mathrm{RCT}, 18$ participants); brushing was carried out by the carers. In the short term, neither toothbrush showed superiority (GI: MD $-0.10,95 \% \mathrm{Cl}-0.77$ to 0.57 ; plaque: $\mathrm{MD} 0.20,95 \% \mathrm{Cl}-0.45$ to $0.85 ; 1 \mathrm{RCT}, 25$ participants; low- to very low-certainty evidence).

Moderate- and low-certainty evidence found no difference between electric and manual toothbrushes for reducing $\mathrm{Gl}$ or plaque, respectively, in the medium term ( $\mathrm{Gl}$ : $\mathrm{MD} 0.02,95 \% \mathrm{Cl}-0.06$ to 0.09 ; plaque: standardised mean difference $0.29,95 \% \mathrm{Cl}-0.07$ to $0.65 ; 2$ RCTs, 120 participants). Short-term findings were inconsistent (4 RCTs; low- to very low-certainty evidence).

Low-certainty evidence suggested training carers in oral hygiene care had no detectable effect on levels of $\mathrm{Gl}$ or plaque in the medium term (GI: $\mathrm{MD}-0.09,95 \% \mathrm{Cl}-0.63$ to 0.45 ; plaque: $\mathrm{MD}-0.07,95 \% \mathrm{Cl}-0.26$ to $0.13 ; 2 \mathrm{RCTs}, 99$ participants). Low-certainty evidence suggested oral hygiene knowledge of carers was better in the medium term after training (MD 0.69, 95\% Cl 0.31 to 1.06; 2 RCTs, 189 participants); this was not found in the short term, and results for changes in behaviour, attitude and self-efficacy were mixed.

One RCT (10 participants) found that training people with ID in oral hygiene care reduced plaque but not GI in the short term (GI: MD -0.28, $95 \% \mathrm{Cl}-0.90$ to 0.34 ; plaque: $\mathrm{MD}-0.47,95 \% \mathrm{Cl}-0.92$ to -0.02 ; very low-certainty evidence).

One RCT (304 participants) found that scheduled dental recall visits (at 1-, 3- or 6-month intervals) plus supervised daily toothbrushing were more likely than usual care to reduce GI (pocketing but not bleeding) and plaque in the long term (low-certainty evidence).

One RCT (29 participants) found that motivating people with ID about oral hygiene by discussing photographs of their teeth with plaque highlighted by a plaque-disclosing agent, did not reduce plaque in the medium term (very low-certainty evidence).

One RCT (80 participants) found daily toothbrushing by dental students was more effective for reducing plaque in people with ID than once- or twice-weekly toothbrushing in the short term (low-certainty evidence).

A benefit to gingival health was found by one NRS that evaluated toothpaste with a plaque-disclosing agent and one that evaluated individualised oral care plans (very low-certainty evidence).

Most studies did not report adverse effects; of those that did, only one study considered them as a formal outcome. Some studies reported participant difficulties using the electric or special manual toothbrushes.

\section{Authors' conclusions}

Although some oral hygiene interventions for people with ID show benefits, the clinical importance of these benefits is unclear. The evidence is mainly low or very low certainty. Moderate-certainty evidence was available for only one finding: electric and manual toothbrushes were similarly effective for reducing gingival inflammation in people with ID in the medium term. Larger, higher-quality RCTs are recommended to endorse or refute the findings of this review. In the meantime, oral hygiene care and advice should be based on professional expertise and the needs and preferences of the individual with ID and their carers.

\section{PLAIN LANGUAGE SUMMARY}

\section{Oral hygiene programmes for people with intellectual disabilities}

\section{Review question}

How effective are oral hygiene programmes for people with intellectual disabilities?

\section{Background}

The removal of dental plaque by daily toothbrushing plays a major role in preventing tooth decay and gum disease, the two main causes of tooth loss. Toothbrushing is a skill that can be difficult for people with ID; they may require help and people who care for them may need training in how to help them. 


\section{Study characteristics}

We searched for studies up to 4 February 2019. This review included 34 studies that involved 1795 people with ID and 354 carers. Nineteen studies randomly allocated participants to two or more groups (i.e. randomised controlled trials (RCTs), and 15 were non-randomised studies (NRS).

The studies assessed different ways to improve the oral hygiene of people with ID: special manual toothbrushes; electric toothbrushes; oral hygiene training for carers; oral hygiene training for people with ID; varying the scheduled intervals between dental visits and supervising toothbrushing; using discussion of clinical photographs as a motivator; varying how frequently the teeth of people with ID were brushed; using a plaque-disclosing agent and using individualised oral care plans.

The studies evaluated gingival inflammation (red and swollen gums) and plaque. Some studies evaluated carer knowledge, behaviour, attitude and self-efficacy (belief in their competence) in terms of oral hygiene, as well as the oral hygiene behaviour and skills of people with ID. Tooth decay and quality of life were not measured. We grouped the studies according to when the outcomes were measured: short term (six weeks or less), medium term (between six weeks and 12 months) and long term (more than 12 months).

\section{Key results}

A special manual toothbrush (the Superbrush), used by carers, may be better at reducing levels of gingival inflammation and possibly plaque in people with ID than an ordinary manual toothbrush in the medium term, though this was not seen in the short term.

We found no difference between electric and manual toothbrushes used by people with ID or their carers in terms of gingival inflammation or plaque in the medium term, and the short-term results were unclear.

Training carers to brush the teeth of people with ID may have improved carers' oral hygiene knowledge in the medium term.

Training people with ID to brush their own teeth may have reduced the amount of plaque on their teeth in the short term.

Regularly scheduled dental recall visits and carers supervising toothbrushing between visits may have been more likely than usual care to reduce gingival inflammation and plaque in the long term.

Discussing clinical photographs of plaque on participants' teeth shown up by a disclosing agent, to motivate them to better toothbrushing did not seem to reduce plaque.

Daily toothbrushing by a dental student may be more effective for reducing plaque levels in the short term than once or twice weekly professional toothbrushing.

Toothpaste with a plaque-disclosing agent and individualised oral care plans were each evaluated in one nonrandomised study that suggested they may be beneficial.

Only one study set out to formally measure negative side effects; however, most studies commented that there were none. Some studies found that some people had difficulties with the electric or special manual toothbrushes.

\section{Certainty of the evidence}

Although some oral hygiene interventions for people with ID show scientific evidence of benefits, what these benefits actually mean for an individual's oral hygiene or oral health is unclear. The certainty of the evidence is mainly low or very low so future research may change our findings. Moderate-certainty evidence is available for only one finding: electric and manual toothbrushes are probably similarly effective for reducing gingival inflammation in people with ID in the medium term. More and better research is needed to fully evaluate interventions that show promise for improving the oral hygiene of people with ID, and to confirm which interventions are ineffective. In the meantime, changes to current habits based on this review should be made cautiously, and decisions about oral hygiene care should be based on professional expertise and the needs and preferences of people with ID and their carers. 
SUMMARY OF FINDINGS

Summary of findings for the main comparison. Special manual toothbrush compared to conventional manual toothbrush for people with intellectual disabilities

Special manual toothbrush compared to conventional manual toothbrush for people with ID

Population: children and adults with mixed levels of ID

Setting: home, residential, day care, school, mixed or not specified

Intervention: special manual toothbrush (self, carer or carer-assisted brushing)

Comparison: conventional manual toothbrush (self, carer or carer-assisted brushing)

\begin{tabular}{|c|c|c|c|c|c|c|}
\hline \multirow[t]{2}{*}{ Outcomes } & \multicolumn{2}{|c|}{$\begin{array}{l}\text { Anticipated absolute effects }{ }^{\star}(95 \% \\
\mathrm{Cl})\end{array}$} & \multirow[t]{2}{*}{$\begin{array}{l}\text { Relative effect } \\
(95 \% \mathrm{Cl})\end{array}$} & \multirow{2}{*}{$\begin{array}{l}\text { Number of par- } \\
\text { ticipants } \\
\text { (studies) }\end{array}$} & \multirow{2}{*}{$\begin{array}{l}\text { Certainty of } \\
\text { the evidence } \\
\text { (GRADE) }\end{array}$} & \multirow[t]{2}{*}{ Comments } \\
\hline & $\begin{array}{l}\text { Risk with con- } \\
\text { ventional manu- } \\
\text { al toothbrush }\end{array}$ & $\begin{array}{l}\text { Risk with spe- } \\
\text { cial manual } \\
\text { toothbrush }\end{array}$ & & & & \\
\hline $\begin{array}{l}\text { Gingival inflammation short } \\
\text { term (<6 weeks) } \\
\text { assessed with: Saxer \& Muhle- } \\
\text { mann Bleeding Index } \\
\text { Scale: } 0-3 \text { (lower score means } \\
\text { less inflammation) } \\
\text { Follow-up: mean } 21 \text { days }\end{array}$ & $\begin{array}{l}\text { Mean gingival in- } \\
\text { flammation short } \\
\text { term was } 1.21 \text { (SD } \\
0.85 \text { ) }\end{array}$ & $\begin{array}{l}\text { MD } 0.10 \text { lower } \\
\text { ( } 0.77 \text { lower to } \\
0.57 \text { higher) }\end{array}$ & - & $\begin{array}{l}25 \\
(1 \mathrm{RCT})\end{array}$ & $\begin{array}{l}\oplus \oplus \odot \ominus \\
\text { Low }^{a}\end{array}$ & $\begin{array}{l}\text { There was no evidence of a difference } \\
\text { between the toothbrushes in the short } \\
\text { term. Two cross-over RCTs ( } 126 \text { partici- } \\
\text { pants) showed similar results. }\end{array}$ \\
\hline $\begin{array}{l}\text { Gingival inflammation } \\
\text { medium term ( } 6 \text { weeks to } 12 \\
\text { months) } \\
\text { assessed with: Gingival Bleed- } \\
\text { ing Index } \% \text { of positive sites } \\
\text { Scale: } 1-100 \text { (lower score } \\
\text { means less inflammation) } \\
\text { Follow-up: mean } 4 \text { months }\end{array}$ & $\begin{array}{l}\text { Mean gingival } \\
\text { inflammation } \\
\text { medium term } \\
\text { was } 18.3 \text { (SD 16.2) }\end{array}$ & $\begin{array}{l}\text { MD } 12.4 \text { lower } \\
\text { (24.31 lower to } \\
0.49 \text { lower) }\end{array}$ & - & $\begin{array}{l}18 \\
(1 \mathrm{RCT})\end{array}$ & $\begin{array}{l}\oplus \ominus \ominus \ominus \\
\text { Very low } b\end{array}$ & $\begin{array}{l}\text { Compared to a conventional tooth- } \\
\text { brush, a special manual toothbrush } \\
\text { (Superbrush) may have reduced gingi- } \\
\text { val inflammation medium term when } \\
\text { used by a carer for people with ID who } \\
\text { were reliant on other people to carry } \\
\text { out their oral hygiene. }\end{array}$ \\
\hline $\begin{array}{l}\text { Plaque short term } \\
\text { assessed with: Silness \& Löe } \\
\text { Plaque Index } \\
\text { Scale: } 0 \text {-3 (lower score means } \\
\text { less plaque) } \\
\text { Follow-up: mean } 21 \text { days }\end{array}$ & $\begin{array}{l}\text { Mean plaque } \\
\text { short term was } \\
1.02 \text { (SD 0.85) }\end{array}$ & $\begin{array}{l}\text { MD } 0.2 \text { higher } \\
\text { (-0.45 lower to } \\
0.85 \text { higher) }\end{array}$ & - & $\begin{array}{l}25 \\
(1 \mathrm{RCT})\end{array}$ & $\begin{array}{l}\oplus \ominus \ominus \ominus \\
\text { Very lowc }\end{array}$ & $\begin{array}{l}\text { There was no evidence of a difference } \\
\text { between the toothbrushes in the short } \\
\text { term. Three other short-term RCTs } \\
\text { (141 participants) that could not be } \\
\text { combined in meta-analysis, had incon- } \\
\text { sistent findings. }\end{array}$ \\
\hline
\end{tabular}




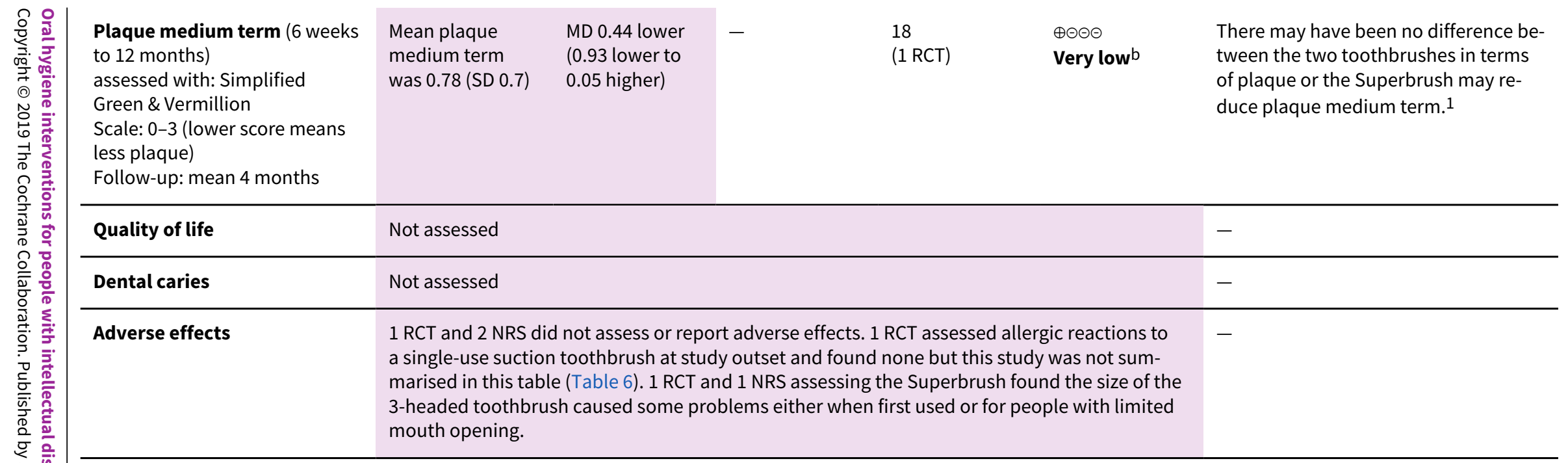

${ }^{*}$ The risk in the intervention group (and its 95\% confidence interval) is based on the assumed risk in the comparison group and the relative effect of the intervention (and its $95 \% \mathrm{Cl})$.

CI: confidence interval; ID: intellectual disability; MD: mean difference; NRS: non-randomised study; RCT: randomised controlled trial; SD: standard deviation.

\section{GRADE Working Group grades of evidence}

High certainty: we are very confident that the true effect lies close to that of the estimate of the effect.

Moderate certainty: we are moderately confident in the effect estimate: the true effect is likely to be close to the estimate of the effect, but there is a possibility that it is substantially different.

Low certainty: our confidence in the effect estimate is limited: the true effect may be substantially different from the estimate of the effect.

Very low certainty: we have very little confidence in the effect estimate: the true effect is likely to be substantially different from the estimate of effect.

aDowngraded two levels due to imprecision: wide confidence intervals and findings were based on one study with only 25 participants.

bDowngraded two levels for imprecision and one for study design: based on only one study with 18 participants, in which assessments were not calibrated and baseline scores were different, and the confidence intervals were wide.

cDowngraded three levels due to imprecision: wide confidence intervals and based on one study with only 18 participants. In addition, 3 other RCTS could not be combined in meta-analysis and had mixed findings.

1One NRS (24 participants) showed no evidence of a difference between toothbrushes for plaque in the medium term..

Summary of findings 2. Electric toothbrush compared to manual toothbrush for people with intellectual disabilities

\section{Electric toothbrush compared to manual toothbrush for people with ID}

Population: children and adults with mixed levels of ID 
Setting: home, residential, day care, school, mixed or not specified

Intervention: electric toothbrush (self, carer or carer-assisted brushing)

Comparison: manual toothbrush (self, carer or carer-assisted brushing)

\begin{tabular}{|c|c|c|c|c|c|c|}
\hline \multirow[t]{2}{*}{ Outcomes } & \multicolumn{2}{|c|}{$\begin{array}{l}\text { Anticipated absolute effects } \\
(95 \% \mathrm{Cl})\end{array}$} & \multirow[t]{2}{*}{$\begin{array}{l}\text { Relative effect } \\
(95 \% \mathrm{CI})\end{array}$} & \multirow{2}{*}{$\begin{array}{l}\text { Number of par- } \\
\text { ticipants } \\
\text { (studies) }\end{array}$} & \multirow{2}{*}{$\begin{array}{l}\text { Certainty of } \\
\text { the evidence } \\
\text { (GRADE) }\end{array}$} & \multirow[t]{2}{*}{ Comments } \\
\hline & $\begin{array}{l}\text { Risk with man- } \\
\text { ual toothbrush }\end{array}$ & $\begin{array}{l}\text { Risk with elec- } \\
\text { tric toothbrush }\end{array}$ & & & & \\
\hline $\begin{array}{l}\text { Gingival inflammation } \\
\text { short term (<6 weeks) } \\
\text { assessed with: assorted } \\
\text { gingival indices } \\
\text { Scale: } 0-4 \text { (lower score } \\
\text { means less inflammation) } \\
\text { Follow-up: } 2-4 \text { weeks }\end{array}$ & - & - & - & 252 (4 RCTs) & $\begin{array}{l}\oplus \oplus \odot \odot \\
\mathbf{L o w}^{a}\end{array}$ & $\begin{array}{l}\text { Four studies showed no significant differ- } \\
\text { ences between toothbrushes for gingival in- } \\
\text { flammation in the short term. Data from the } \\
4 \text { studies could not be combined in a meta- } \\
\text { analysis. }{ }^{1}\end{array}$ \\
\hline $\begin{array}{l}\text { Gingival inflammation } \\
\text { medium term (6 weeks to } \\
12 \text { months) } \\
\text { assessed with: assorted } \\
\text { gingival indices } \\
\text { Scale: } 0 \text {-3 (lower score } \\
\text { means less inflammation) } \\
\text { Follow-up: mean } 6 \\
\text { months }\end{array}$ & $\begin{array}{l}\text { Mean gingival } \\
\text { inflammation } \\
\text { medium term } \\
\text { ranged from } \\
1.24 \text { to } 1.9\end{array}$ & $\begin{array}{l}\text { MD } 0.02 \text { higher } \\
\text { ( } 0.06 \text { lower to } \\
0.09 \text { higher) }\end{array}$ & - & $\begin{array}{l}120 \\
(2 \mathrm{RCTs})\end{array}$ & $\begin{array}{l}\oplus \oplus \oplus \ominus \\
\text { Moderate }^{b}\end{array}$ & $\begin{array}{l}\text { There was little to no difference between } \\
\text { toothbrushes for gingival inflammation medi- } \\
\text { um term. Another RCT ( } 100 \text { participants) } \\
\text { showed findings in favour of the electric } \\
\text { toothbrush ( } 53 \% \text { versus } 31 \% \text { improvement), } \\
\text { but it used a more subjective assessment of } \\
\text { GI.2 }\end{array}$ \\
\hline $\begin{array}{l}\text { Plaque short term }(<6 \\
\text { weeks) } \\
\text { assessed with: assorted } \\
\text { plaque indices } \\
\text { Scale: } 0-5 \text { (lower score } \\
\text { means less plaque) } \\
\text { Follow-up: } 1-4 \text { weeks }\end{array}$ & - & - & - & 247 (4 RCTs) & $\begin{array}{l}\oplus \ominus \Theta \ominus \\
\text { Very low }\end{array}$ & $\begin{array}{l}\text { We were uncertain about the effect of using } \\
\text { either toothbrush on plaque short term. None } \\
\text { of the studies presented data that could be } \\
\text { included in a meta-analysis. } 2 \text { studies ( } 69 \text { par- } \\
\text { ticipants) showed some benefit in favour of } \\
\text { the electric toothbrush; } 2 \text { studies ( } 178 \text { partici- } \\
\text { pants) showed no difference between the two } \\
\text { toothbrushes. } 3\end{array}$ \\
\hline $\begin{array}{l}\text { Plaque medium term (6 } \\
\text { weeks to } 12 \text { months) } \\
\text { assessed with: assorted } \\
\text { plaque indices } \\
\text { Scale: } 0-3 \text { (lower score } \\
\text { means less plaque) }\end{array}$ & $\begin{array}{l}\text { Mean plaque } \\
\text { medium term } \\
\text { ranged from } \\
1.12 \text { to } 1.55\end{array}$ & $\begin{array}{l}\text { SMD } 0.29 \text { higher } \\
\text { ( } 0.07 \text { lower to } \\
0.65 \text { higher) }\end{array}$ & - & $\begin{array}{l}120 \\
\text { (2 RCTs) }\end{array}$ & $\begin{array}{l}\oplus \oplus \ominus \ominus \\
\text { Lowd }\end{array}$ & $\begin{array}{l}\text { There was no clear difference between the } \\
\text { toothbrushes for plaque medium term. } 1 \text { RCT } \\
\text { (100 participants) not included in the meta- } \\
\text { analysis, which used a subjective assessment } \\
\text { of oral hygiene, showed an improvement in } \\
\text { favour of the electric toothbrush. }{ }^{4}\end{array}$ \\
\hline
\end{tabular}




Quality of life Not assessed

No studies assessed quality of life changes formally, although some made comments in the results or discussion sections of the reports.

\begin{tabular}{ll}
\hline Dental caries & Not assessed \\
\hline Adverse effects & Seven studies did not assess or report adverse effects and one reported no adverse effects. \\
1 NRS reported discomfort when brushing, difficulty controlling the force and position of the \\
electric toothbrush, and the electric toothbrush being out of order; 2 RCTs and 1 NRS report- \\
ed participants being frightened when the electric toothbrush was first used. 1 RCT reported \\
participants being dismissed from the study, but no clear reason was provided.
\end{tabular}

${ }^{\star}$ The risk in the intervention group (and its $95 \%$ confidence interval) is based on the assumed risk in the comparison group and the relative effect of the intervention (and its $95 \% \mathrm{Cl})$.

CI: confidence interval; ID: intellectual disability; MD: mean difference; NRS: non-randomised study; RCT: randomised controlled trial; SD: standard deviation; SMD: standardised mean difference.

\section{GRADE Working Group grades of evidence}

High certainty: we are very confident that the true effect lies close to that of the estimate of the effect.

Moderate certainty: we are moderately confident in the effect estimate: the true effect is likely to be close to the estimate of the effect, but there is a possibility that it is substantially different.

Low certainty: our confidence in the effect estimate is limited: the true effect may be substantially different from the estimate of the effect.

Very low certainty: we have very little confidence in the effect estimate: the true effect is likely to be substantially different from the estimate of effect.

aDowngraded two levels due to study design as three studies at high risk of bias (blinding of participants and personnel was not possible in three of the studies; one study also had incomplete outcome data).

bDowngraded one level due to study design as studies at unclear risk of bias.

cDowngraded two levels for study design and one level for heterogeneity: three studies at high risk of bias (blinding of participants and personnel was not possible in three of the studies), and findings inconsistent between studies.

dDowngraded one level for study design and one level for imprecision: studies at unclear risk of bias and result imprecise.

1 One NRS (23 participants) similarly found no difference between toothbrushes for gingival inflammation in the short term.

2Four NRS (144 participants) had inconsistent findings at 5 to 9 months for gingival inflammation in the medium term; data not suitable for meta-analysis. 1 NRS (23 participants) found no effect at 16 months (MD $0.00,95 \% \mathrm{Cl}-0.15$ to 0.15 ).

3 One NRS (23 participants) found no difference between toothbrushes for plaque in the short term.

${ }^{4}$ Five NRS (173 participants) found broadly similar results to the meta-analysis, showing little or no effect on plaque in the medium term. 


\begin{tabular}{|c|c|c|c|c|c|c|}
\hline \multicolumn{7}{|c|}{ Training of carers compared to no training of carers for people with ID } \\
\hline \multicolumn{7}{|c|}{ Population: people with mixed levels of ID (age range unclear) } \\
\hline \multicolumn{7}{|l|}{ Setting: residential } \\
\hline \multicolumn{7}{|l|}{ Intervention: training of carers } \\
\hline \multicolumn{7}{|l|}{ Comparison: no training of carers } \\
\hline \multirow[t]{2}{*}{ Outcomes } & \multicolumn{2}{|c|}{$\begin{array}{l}\text { Anticipated absolute effects* } \\
(95 \% \mathrm{Cl})\end{array}$} & \multirow[t]{2}{*}{$\begin{array}{l}\text { Relative effect } \\
(95 \% \mathrm{CI})\end{array}$} & \multirow{2}{*}{$\begin{array}{l}\text { Number of par- } \\
\text { ticipants } \\
\text { (studies) }\end{array}$} & \multirow{2}{*}{$\begin{array}{l}\text { Certainty of } \\
\text { the evidence } \\
\text { (GRADE) }\end{array}$} & \multirow[t]{2}{*}{ Comments } \\
\hline & $\begin{array}{l}\text { Risk with no } \\
\text { carer training }\end{array}$ & $\begin{array}{l}\text { Risk with carer } \\
\text { training }\end{array}$ & & & & \\
\hline $\begin{array}{l}\text { Gingival inflammation short term }(< \\
6 \text { weeks) }\end{array}$ & \multicolumn{5}{|l|}{ Not assessed } & - \\
\hline $\begin{array}{l}\text { Gingival inflammation medium term } \\
\text { (6 weeks to } 12 \text { months) } \\
\text { assessed with: Modified Silness \& Löe } \\
\text { Gingival Index } \\
\text { Scale: } 0-3 \text { (lower score means less in- } \\
\text { flammation) } \\
\text { Follow-up: } 8 \text { weeks to } 9 \text { months }\end{array}$ & $\begin{array}{l}\text { Mean gingival } \\
\text { inflammation } \\
\text { medium term } \\
\text { ranged from } 1.2 \\
\text { to } 2.47\end{array}$ & $\begin{array}{l}\text { MD } 0.09 \text { lower } \\
\text { ( } 0.63 \text { lower to } \\
0.45 \text { higher) }\end{array}$ & - & $\begin{array}{l}99 \\
(2 \text { RCTs) }\end{array}$ & $\begin{array}{l}\oplus \oplus \ominus \ominus \\
\text { Lowa }^{\mathrm{a}, \mathrm{b}}\end{array}$ & $\begin{array}{l}\text { Training of carers may not have } \\
\text { made a difference to gingival in- } \\
\text { flammation. Findings for the } 2 \\
\text { studies were inconsistent. }\end{array}$ \\
\hline Plaque short term (< 6 weeks) & \multicolumn{5}{|l|}{ Not assessed } & - \\
\hline $\begin{array}{l}\text { Plaque medium term ( } 6 \text { weeks to } 12 \\
\text { months) } \\
\text { assessed with: Modified Silness \& Löe } \\
\text { Plaque Index } \\
\text { Scale: } 0-3 \text { (lower score means less } \\
\text { plaque) } \\
\text { Follow-up: } 8 \text { weeks to } 9 \text { months }\end{array}$ & $\begin{array}{l}\text { Mean plaque } \\
\text { medium term } \\
\text { ranged from } 1.4 \\
\text { to } 1.89\end{array}$ & $\begin{array}{l}\text { MD } 0.07 \text { lower } \\
\text { ( } 0.26 \text { lower to } \\
0.13 \text { higher) }\end{array}$ & - & $\begin{array}{l}99 \\
(2 \mathrm{RCTS})\end{array}$ & $\begin{array}{l}\oplus \oplus \ominus \ominus \\
\text { Lowa,c }\end{array}$ & $\begin{array}{l}\text { Training of carers may not have } \\
\text { reduced the plaque levels for } \\
\text { people with ID medium term. }{ }^{1}\end{array}$ \\
\hline $\begin{array}{l}\text { Knowledge medium term ( } 6 \text { weeks to } \\
12 \text { months) } \\
\text { assessed with: questionnaire } \\
\text { Scale } 0-10 \text { (higher score means in- } \\
\text { creased knowledge) } \\
\text { Follow-up: } 8 \text { weeks to } 11 \text { months }\end{array}$ & $\begin{array}{l}\text { Mean knowl- } \\
\text { edge medium } \\
\text { term ranged } \\
\text { from } 6.8 \text { to } 7.86\end{array}$ & $\begin{array}{l}\text { MD } 0.69 \text { higher } \\
\text { ( } 0.31 \text { higher to } \\
1.06 \text { higher) }\end{array}$ & - & $\begin{array}{l}189 \\
(2 \mathrm{RCTs})\end{array}$ & $\begin{array}{l}\oplus \oplus \ominus \ominus \\
\text { Low }^{\mathrm{a}, \mathrm{d}}\end{array}$ & $\begin{array}{l}\text { Training of carers may have re- } \\
\text { sulted in a moderate increase in } \\
\text { oral healthcare knowledge medi- } \\
\text { um term. } 1 \text { RCT ( } 24 \text { participants) } \\
\text { not included in the meta-analy- } \\
\text { sis found no clear difference be- } \\
\text { tween groups. }\end{array}$ \\
\hline
\end{tabular}




\begin{tabular}{|c|c|c|c|c|c|c|}
\hline \multicolumn{2}{|r|}{$\begin{array}{l}\text { Behaviour, attitude and self-efficacy } \\
\text { (BAS) medium term } \\
\text { (6 weeks to } 12 \text { months) assessed with: } \\
\text { questionnaire } \\
\text { Scale: } 0-14 \text { (higher score means im- } \\
\text { proved BAS) } \\
\text { Follow-up: } 8 \text { weeks to } 11 \text { months }\end{array}$} & $\begin{array}{l}\text { Mean BAS } \\
\text { ranged from } 4.7 \\
\text { to } 5.42\end{array}$ & $\begin{array}{l}\text { MD } 0.15 \text { higher } \\
\text { ( } 0.80 \text { lower to } \\
1.10 \text { higher) }\end{array}$ & $\begin{array}{l}189 \\
(2 \mathrm{RCTS})\end{array}$ & $\begin{array}{l}\oplus \ominus \ominus \ominus \\
\text { Very low } a, b, d\end{array}$ & $\begin{array}{l}\text { Training of carers may not have } \\
\text { an effect on their BAS in the } \\
\text { medium term. } \\
\text { In the short term, } 1 \text { RCT (193 par- } \\
\text { ticipants) assessed behaviour } \\
\text { and reported a strong benefit for } \\
\text { training.2 }\end{array}$ \\
\hline 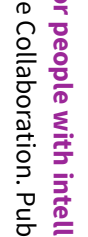 & Quality of life & \multicolumn{4}{|l|}{ Not assessed } & $\begin{array}{l}\text { No studies formally reported } \\
\text { quality of life measures, though } \\
\text { some studies commented on } \\
\text { quality of life issues in the discus- } \\
\text { sion and conclusion section of } \\
\text { the reports. }\end{array}$ \\
\hline 敢 & Dental caries & \multicolumn{4}{|l|}{ Not assessed } & - \\
\hline 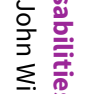 & Adverse effects & \multicolumn{4}{|c|}{$\begin{array}{l}1 \mathrm{RCT} \text { reported there were no adverse effects. The other studies did not report adverse ef- } \\
\text { fects. }\end{array}$} & - \\
\hline
\end{tabular}

${ }^{\star}$ The risk in the intervention group (and its $95 \%$ confidence interval) is based on the assumed risk in the comparison group and the relative effect of the intervention (and its $95 \% \mathrm{Cl})$.

BAS: behaviour, attitude and self-efficacy; CI: confidence interval; ID: intellectual disability; ITS: interrupted time series; MD: mean difference; NRCT: non-randomised controlled trial; NRS: non-randomised study; RCT: randomised controlled trial; SD: standard deviation.

\section{GRADE Working Group grades of evidence}

High certainty: we are very confident that the true effect lies close to that of the estimate of the effect.

Moderate certainty: we are moderately confident in the effect estimate: the true effect is likely to be close to the estimate of the effect, but there is a possibility that it is substantially different.

Low certainty: our confidence in the effect estimate is limited: the true effect may be substantially different from the estimate of the effect.

Very low certainty: we have very little confidence in the effect estimate: the true effect is likely to be substantially different from the estimate of effect.

aDowngraded one level due to study design as studies at high risk of bias (one study was a cluster RCT).

bDowngraded one level due to heterogeneity as findings were inconsistent between studies.

cDowngraded one level due to study design as index was modified to an extent that it may have impacted on the results.

dDowngraded one level due to study design as carer outcomes had high levels of attrition in both studies.

1One NRCT (34 participants) found plaque levels of people with ID were reduced after training of their carers, short term, particularly if carers were made accountable for their performance. 1 ITS (11 participants) found broadly consistent findings to the NRCT in the medium term.

2Two NRS (15 participants) assessing the behaviour of the carers and people with ID showed evidence in favour of training for all outcomes assessed. 
Summary of findings 4. Oral hygiene training compared to no oral hygiene training for people with intellectual disabilities

Oral hygiene training compared to no oral hygiene training for people with ID

Population: children and adults with mild to profound ID

Setting: residential or school

Intervention: oral hygiene training (toothbrushing routine)

Comparison: no oral hygiene training

\begin{tabular}{|c|c|c|c|c|c|c|}
\hline \multirow[t]{2}{*}{ Outcomes } & \multicolumn{2}{|c|}{$\begin{array}{l}\text { Anticipated absolute effects* }{ }^{\star}(95 \% \\
\mathrm{CI})\end{array}$} & \multirow[t]{2}{*}{$\begin{array}{l}\text { Relative effect } \\
(95 \% \mathrm{CI})\end{array}$} & \multirow{2}{*}{$\begin{array}{l}\text { Number of par- } \\
\text { ticipants } \\
\text { (studies) }\end{array}$} & \multirow{2}{*}{$\begin{array}{l}\text { Certainty of } \\
\text { the evidence } \\
\text { (GRADE) }\end{array}$} & \multirow[t]{2}{*}{ Comments } \\
\hline & $\begin{array}{l}\text { Risk with no oral } \\
\text { hygiene training }\end{array}$ & $\begin{array}{l}\text { Risk with oral } \\
\text { hygiene train- } \\
\text { ing }\end{array}$ & & & & \\
\hline $\begin{array}{l}\text { Gingival inflammation short term }(<6 \\
\text { weeks) } \\
\text { assessed with: Silness \& Löe Gingival Index } \\
\text { Scale: } 0-3 \text { (lower score means less gingival } \\
\text { inflammation) } \\
\text { Follow-up: } 6-7 \text { weeks }\end{array}$ & $\begin{array}{l}\text { The mean gingi- } \\
\text { val inflammation } \\
\text { short term was } \\
1.26 \text { (SD 0.46) }\end{array}$ & $\begin{array}{l}\text { MD } 0.28 \text { lower } \\
\text { ( } 0.90 \text { lower to } \\
0.34 \text { higher) }\end{array}$ & - & $\begin{array}{l}10 \\
(1 \mathrm{RCT})\end{array}$ & $\begin{array}{l}\oplus \odot \odot \ominus \\
\text { Very low } a\end{array}$ & $\begin{array}{l}\text { There was no evidence of } \\
\text { a difference between the } \\
\text { groups in the short term. }{ }^{1}\end{array}$ \\
\hline $\begin{array}{l}\text { Gingival inflammation medium term ( } 6 \\
\text { weeks to } 12 \text { months) }\end{array}$ & \multicolumn{5}{|l|}{ Not assessed } & - \\
\hline $\begin{array}{l}\text { Plaque short term (<6 weeks) } \\
\text { assessed with: Green \& Vermillion Plaque } \\
\text { Index } \\
\text { Scale: 0-3 (lower score means less plaque) } \\
\text { Follow-up: 6-7 weeks }\end{array}$ & $\begin{array}{l}\text { The mean plaque } \\
\text { short term was } \\
0.82(0.24)\end{array}$ & $\begin{array}{l}\text { MD } 0.47 \text { lower } \\
\text { (0.92 lower to } \\
0.02 \text { lower) }\end{array}$ & - & $\begin{array}{l}10 \\
(1 \mathrm{RCT})\end{array}$ & $\begin{array}{l}\oplus \odot \odot \ominus \\
\text { Very low } a\end{array}$ & $\begin{array}{l}\text { Training of people with } \\
\text { ID may have resulted in } \\
\text { a moderate reduction in } \\
\text { plaque short term. An RCT } \\
\text { ( } 26 \text { participants) not in- } \\
\text { cluded in the meta-analy- } \\
\text { sis had similar findings. }{ }^{1}\end{array}$ \\
\hline $\begin{array}{l}\text { Plaque medium term ( } 6 \text { weeks to } 12 \\
\text { months) }\end{array}$ & \multicolumn{5}{|l|}{ Not assessed } & - \\
\hline Knowledge & \multicolumn{5}{|l|}{ Not assessed } & - \\
\hline $\begin{array}{l}\text { Behaviour, attitude and self-efficacy } \\
\text { medium and long term ( } 6 \text { weeks to }>12 \\
\text { months) }\end{array}$ & \multicolumn{3}{|c|}{$\begin{array}{l}\text { Results were not presented in a format that could be in- } \\
\text { cluded in a meta-analysis. }\end{array}$} & 59 (4 NRS) & $\begin{array}{l}\oplus \odot \ominus \ominus \\
\text { Very low } b\end{array}$ & $\begin{array}{l}3 \text { NRS ( } 56 \text { participants) } \\
\text { reported an increase in } \\
\text { toothbrushing after train- }\end{array}$ \\
\hline
\end{tabular}




\begin{tabular}{lll}
\hline Quality of life & Not assessed & - \\
\hline Dental caries & Not assessed & - \\
\hline Adverse effects & $\begin{array}{l}\text { 2 RCTs and } 2 \text { NRS reported no adverse effects. In 1 NRS, 1 participant had nausea (considered a } \\
\text { delaying tactic by study investigators), and in another NRS, } 2 \text { participants showed sensitivity to } \\
\text { having their mouths held open. }\end{array}$ & - \\
\hline
\end{tabular}

${ }^{\star}$ The risk in the intervention group (and its $95 \%$ confidence interval) is based on the assumed risk in the comparison group and the relative effect of the intervention (and its $95 \% \mathrm{Cl}$ ).

CI: confidence interval; ID: intellectual disability; MD: mean difference; NRS: non-randomised study; RCT: randomised controlled trial; SD: standard deviation.

\section{GRADE Working Group grades of evidence}

High certainty: we are very confident that the true effect lies close to that of the estimate of the effect.

Moderate certainty: we are moderately confident in the effect estimate: the true effect is likely to be close to the estimate of the effect, but there is a possibility that it is substantially different.

Low certainty: our confidence in the effect estimate is limited: the true effect may be substantially different from the estimate of the effect.

Very low certainty: we have very little confidence in the effect estimate: the true effect is likely to be substantially different from the estimate of effect.

aDowngraded two levels for imprecision and one for study design: based on the findings of one study at unclear risk of bias with only 10 participants and wide confidence intervals. $b$ Downgraded three levels due to study design: based on NRS, with no control groups and all studies were at high risk of bias.

1Four NRS (59 participants) assessed toothbrushing behaviour following skills training of people with ID, 3 of which showed improved toothbrushing behaviour; no measures of gingival inflammation levels were recorded in these studies.

\section{Summary of findings 5. Regularly scheduled dental visits and daily supervised toothbrushing compared to usual care for people with intellectual} disabilities

\section{Scheduled dental recall intervals and supervised toothbrushing compared to usual care for people with ID}

Population: adults (mean age 30.9 years) (level of ID unclear)

Setting: day centre

Intervention: scheduled dental visits (oral hygiene instruction, with or without scale and polish), with supervised daily toothbrushing by carers ("staff provided reminders, encouragement and motivation")

Comparison: usual care ("no specific treatment") 


\begin{tabular}{|c|c|c|c|c|c|c|}
\hline \multirow[t]{2}{*}{ Outcomes } & \multicolumn{2}{|c|}{$\begin{array}{l}\text { Anticipated absolute effects* } \\
(95 \% \mathrm{Cl})\end{array}$} & \multirow[t]{2}{*}{$\begin{array}{l}\text { Relative effect } \\
(95 \% \mathrm{Cl})\end{array}$} & \multirow{2}{*}{$\begin{array}{l}\text { Number of par- } \\
\text { ticipants } \\
\text { (studies) }\end{array}$} & \multirow{2}{*}{$\begin{array}{l}\text { Certainty of } \\
\text { the evidence } \\
\text { (GRADE) }\end{array}$} & \multirow[t]{2}{*}{ Comments } \\
\hline & $\begin{array}{l}\text { Risk with usual } \\
\text { care }\end{array}$ & $\begin{array}{l}\text { Risk with 1- } \\
\text { monthly den- } \\
\text { tal recall inter- } \\
\text { vals }\end{array}$ & & & & \\
\hline $\begin{array}{l}\text { Gingival inflammation short } \\
\text { term }(<6 \text { weeks })\end{array}$ & Not assessed & & & & & - \\
\hline $\begin{array}{l}\text { Gingival pocketing long } \\
\text { term (> } 12 \text { months) } \\
\text { assessed with: WHO techni- } \\
\text { cal report } \\
\text { Scale: } 0-2 \text { (lower score } \\
\text { means less pocketing) } \\
\text { Follow-up: mean } 24 \text { months }\end{array}$ & $\begin{array}{l}\text { The mean gin- } \\
\text { gival pocketing } \\
\text { long term was } \\
0.9 \text { (SD 1.4) }\end{array}$ & $\begin{array}{l}\text { MD } 0.60 \text { lower } \\
\text { (0.97 lower to } \\
0.23 \text { lower) }\end{array}$ & - & $\begin{array}{l}304 \\
(1 \mathrm{RCT})\end{array}$ & $\begin{array}{l}\oplus \oplus \oplus \ominus \\
\text { Low }^{a}\end{array}$ & $\begin{array}{l}\text { One-monthly dental recall intervals and } \\
\text { supervised toothbrushing may have re- } \\
\text { duced gingival pocketing long term. This } \\
\text { was also found for the } 3 \text { - and } 6 \text {-month- } \\
\text { ly recall intervals. Findings were broadly } \\
\text { similar at } 12 \text { and } 18 \text { months. }\end{array}$ \\
\hline $\begin{array}{l}\text { Gingival bleeding long term } \\
\text { (> } 12 \text { months) } \\
\text { assessed with: WHO techni- } \\
\text { cal report } \\
\text { Scale: } 0-1 \text { (lower score } \\
\text { means less bleeding) } \\
\text { Follow-up: mean } 24 \text { months }\end{array}$ & $\begin{array}{l}\text { The mean gin- } \\
\text { gival bleeding } \\
\text { long term was } \\
2.4 \text { (SD 2.0) }\end{array}$ & $\begin{array}{l}\text { MD } 0.20 \text { lower } \\
\text { (0.86 lower to } \\
0.46 \text { higher) }\end{array}$ & - & $\begin{array}{l}304 \\
(1 \mathrm{RCT})\end{array}$ & $\begin{array}{l}\oplus \oplus \ominus \ominus \\
\text { Low }^{a}\end{array}$ & $\begin{array}{l}\text { One-monthly dental recall intervals } \\
\text { showed no evidence of benefit. This was } \\
\text { also found for } 3 \text { - and } 6 \text {-monthly recall in- } \\
\text { tervals. Findings were broadly similar at } \\
12 \text { and } 18 \text { months. }\end{array}$ \\
\hline $\begin{array}{l}\text { Plaque short term (<6 } \\
\text { weeks) }\end{array}$ & Not assessed & & & & & - \\
\hline $\begin{array}{l}\text { Plaque long term (> } 12 \\
\text { months) } \\
\text { assessed with: WHO Plaque } \\
\text { Index } \\
\text { Scale: } 0-3 \text { (lower score } \\
\text { means less plaque) } \\
\text { Follow-up: mean } 24 \text { months }\end{array}$ & $\begin{array}{l}\text { The mean } \\
\text { plaque long } \\
\text { term was } 2.0 \\
\text { (SD 1.9) }\end{array}$ & $\begin{array}{l}\text { MD } 0.70 \text { lower } \\
\text { (1.28 lower to } \\
0.12 \text { lower) }\end{array}$ & - & $\begin{array}{l}304 \\
(1 \mathrm{RCT})\end{array}$ & $\begin{array}{l}\oplus \oplus \ominus \ominus \\
\text { Low }^{a}\end{array}$ & $\begin{array}{l}\text { 1-monthly dental recall intervals may } \\
\text { have reduced plaque levels long term ( } 24 \\
\text { months). This was also found for } 3 \text {-month- } \\
\text { ly recall intervals, but not for } 6 \text {-monthly. } \\
\text { Findings were broadly similar at } 12 \text { and } 18 \\
\text { months. }\end{array}$ \\
\hline Quality of life & Not assessed & & & & & - \\
\hline Dental caries & Not reported & & & & & $\begin{array}{l}\text { The study assessed and reported dental } \\
\text { caries using the DMFT Index at baseline } \\
\text { but did not report assessing DMFT at any } \\
\text { other time points. }\end{array}$ \\
\hline
\end{tabular}


${ }^{*}$ The risk in the intervention group (and its $95 \%$ confidence interval) is based on the assumed risk in the comparison group and the relative effect of the intervention (and its $95 \% \mathrm{Cl}$ ).

CI: confidence interval; DMFT: Decayed, Missing or Filled Teeth; ID: intellectual disability; MD: mean difference; RCT: randomised controlled trial; SD: standard deviation; WHO: World Health Organization.

\section{GRADE Working Group grades of evidence}

High certainty: we are very confident that the true effect lies close to that of the estimate of the effect.

Moderate certainty: we are moderately confident in the effect estimate: the true effect is likely to be close to the estimate of the effect, but there is a possibility that it is substantially different.

Low certainty: our confidence in the effect estimate is limited: the true effect may be substantially different from the estimate of the effect.

Very low certainty: we have very little confidence in the effect estimate: the true effect is likely to be substantially different from the estimate of effect.

${ }^{a}$ Downgraded one level for imprecision and one for study design: based on one study at unclear risk of bias, with wide confidence intervals.

Summary of findings 6. Discussion of clinical photographs as oral hygiene motivators compared to no discussion for people with intellectual disabilities

Discussion of clinical photographs as $\mathrm{OH}$ motivators compared to no discussion of photographs for people with ID

Population: people with ID (level of ID and age unclear)

Setting: day centre

Intervention: discussion of clinical photographs as $\mathrm{OH}$ motivators and $\mathrm{OH}$ instruction at monthly intervals

Comparison: no discussion of clinical photographs, but did receive $\mathrm{OH}$ instruction at monthly intervals

\begin{tabular}{|c|c|c|c|c|c|c|}
\hline \multirow[t]{2}{*}{ Outcomes } & \multicolumn{2}{|c|}{ Anticipated absolute effects* $(95 \% \mathrm{Cl})$} & \multirow{2}{*}{$\begin{array}{l}\text { Relative effect } \\
(95 \% \mathrm{Cl})\end{array}$} & \multirow{2}{*}{$\begin{array}{l}\text { Number of par- } \\
\text { ticipants } \\
\text { (studies) }\end{array}$} & \multirow{2}{*}{$\begin{array}{l}\text { Certainty of } \\
\text { the evidence } \\
\text { (GRADE) }\end{array}$} & \multirow[t]{2}{*}{ Comments } \\
\hline & $\begin{array}{l}\text { Risk with oral } \\
\text { hygiene instruc- } \\
\text { tion (OHI) only }\end{array}$ & $\begin{array}{l}\text { Risk with discus- } \\
\text { sion of clinical } \\
\text { photographs plus } \\
\text { OHI }\end{array}$ & & & & \\
\hline $\begin{array}{l}\text { Gingival inflammation short term }(<6 \\
\text { weeks) }\end{array}$ & Not assessed & & & & & - \\
\hline $\begin{array}{l}\text { Gingival inflammation medium term ( } 6 \\
\text { weeks to } 12 \text { months) }\end{array}$ & Not assessed & & & & & - \\
\hline
\end{tabular}




\begin{tabular}{|c|c|c|c|c|c|c|c|}
\hline 㑒 & Plaque short term ( $<6$ weeks) & Not assessed & & & & & - \\
\hline 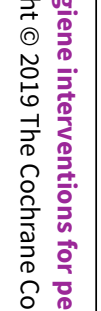 & $\begin{array}{l}\text { Plaque medium term ( } 6 \text { weeks to } 12 \text { months) } \\
\text { assessed with: WHO Plaque Index } \\
\text { Scale: } 0-3 \text { (lower score means less plaque) } \\
\text { Follow-up: mean } 6 \text { months }\end{array}$ & $\begin{array}{l}\text { Mean plaque } \\
\text { medium term } \\
\text { was } 7.4 \text { (SD 2.0) }\end{array}$ & $\begin{array}{l}\text { MD } 0.1 \text { lower } \\
\text { (0.85 lower to } 0.65 \\
\text { higher) }\end{array}$ & - & $\begin{array}{l}29 \\
(1 \mathrm{RCT})\end{array}$ & $\begin{array}{l}\oplus \ominus \ominus \ominus \\
\text { Very low } \text { low }^{a}\end{array}$ & $\begin{array}{l}\text { There was no evi- } \\
\text { dence of a differ- } \\
\text { ence in plaque lev- } \\
\text { els medium term. } \\
\text { Broadly similar find- } \\
\text { ings were observed } \\
\text { for extrinsic plaque } \\
\text { levels. }\end{array}$ \\
\hline$\sum_{\Sigma}^{\overline{0}}$ & Quality of life & Not assessed & & & & & - \\
\hline 焉 & Dental caries & Not assessed & & & & & - \\
\hline 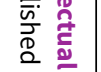 & Adverse effects & None reported & & & & & - \\
\hline
\end{tabular}

*The risk in the intervention group (and its $95 \%$ confidence interval) is based on the assumed risk in the comparison group and the relative effect of the intervention (and its $95 \% \mathrm{Cl})$.

CI: confidence interval; ID: intellectual disability; MD: mean difference; $\mathbf{O H}$ : oral hygiene; $\mathbf{R C T}$ : randomised controlled trial; SD: standard deviation.

\section{GRADE Working Group grades of evidence}

High certainty: we are very confident that the true effect lies close to that of the estimate of the effect.

Moderate certainty: we are moderately confident in the effect estimate: the true effect is likely to be close to the estimate of the effect, but there is a possibility that it is substantially different.

Low certainty: our confidence in the effect estimate is limited: the true effect may be substantially different from the estimate of the effect.

Very low certainty: we have very little confidence in the effect estimate: the true effect is likely to be substantially different from the estimate of effect.

aDowngraded two levels for imprecision and one for study design: single study at high risk of bias, with wide confidence intervals.

\section{Summary of findings 7. Daily toothbrushing compared to twice-weekly or once-weekly toothbrushing for people with intellectual disabilities}

Daily compared to twice-weekly or once-weekly toothbrushing by dental students for people with ID

Population: people aged 15 to 30 years with mixed levels of ID

Setting: residential

Intervention: daily toothbrushing by dental student

Comparison: twice-weekly or once-weekly toothbrushing by dental student 


\begin{tabular}{|c|c|c|c|c|}
\hline Outcomes & Impact & $\begin{array}{l}\text { Number of partici- } \\
\text { pants } \\
\text { (studies) }\end{array}$ & $\begin{array}{l}\text { Certainty of the evi- } \\
\text { dence } \\
\text { (GRADE) }\end{array}$ & Comment \\
\hline $\begin{array}{l}\text { Gingival inflammation short term }(<6 \\
\text { weeks) }\end{array}$ & Not assessed & - & - & - \\
\hline $\begin{array}{l}\text { Gingival inflammation medium term (6 } \\
\text { weeks to } 12 \text { months) }\end{array}$ & Not assessed & - & - & - \\
\hline $\begin{array}{l}\text { Plaque short term (< } \mathbf{6} \text { weeks) } \\
\text { assessed with: Silness \& Löe Gingival Index } \\
\text { (lower score means less plaque) } \\
\text { Follow-up: mean } 21 \text { days }\end{array}$ & $\begin{array}{l}\text { For people with ID whose teeth were } \\
\text { brushed for them by dental profession- } \\
\text { als, brushing daily most likely reduced gin- } \\
\text { gival inflammation more effectively than } \\
\text { twice-weekly and even more effectively } \\
\text { than once-weekly, in the short term. }\end{array}$ & $\begin{array}{l}80 \\
(1 \mathrm{RCT})\end{array}$ & $\begin{array}{l}\oplus \oplus \odot \ominus \\
\text { Low }^{a}\end{array}$ & $\begin{array}{l}\text { This study also com- } \\
\text { pared electric and } \\
\text { manual toothbrush- } \\
\text { es (findings reported } \\
\text { above) }\end{array}$ \\
\hline Plaque medium term ( 6 weeks to 12 months) & Not assessed & - & - & \\
\hline Quality of life & Not assessed & - & - & - \\
\hline Dental caries & Not assessed & - & - & - \\
\hline Adverse effects & None reported & - & - & - \\
\hline
\end{tabular}

\section{GRADE Working Group grades of evidence}

High certainty: we are very confident that the true effect lies close to that of the estimate of the effect.

Moderate certainty: we are moderately confident in the effect estimate: the true effect is likely to be close to the estimate of the effect, but there is a possibility that it is substantially different.

Low certainty: our confidence in the effect estimate is limited: the true effect may be substantially different from the estimate of the effect.

Very low certainty: we have very little confidence in the effect estimate: the true effect is likely to be substantially different from the estimate of effect.

${ }^{a}$ Downgraded two levels for imprecision: single small study with no standard deviation data reported.

Summary of findings 8 . Toothpaste with a plaque-disclosing agent compared to a conventional toothpaste for children with intellectual disabilities

Toothpaste with a plaque-disclosing agent compared to conventional toothpaste for children with ID

Population: children aged 7 to 13 years with Down Syndrome (mild to moderate levels of ID) 


\begin{tabular}{|c|c|c|c|c|}
\hline Outcomes & Impact & $\begin{array}{l}\text { Number of partici- } \\
\text { pants } \\
\text { (studies) }\end{array}$ & $\begin{array}{l}\text { Certainty of the evi- } \\
\text { dence } \\
\text { (GRADE) }\end{array}$ & Comment \\
\hline $\begin{array}{l}\text { Gingival inflammation short term (< } 6 \text { weeks) } \\
\text { assessed with: Ainamo \& Bay Gingival Bleeding Index } \\
\text { (lower score means less inflammation) } \\
\text { Follow-up: mean } 10 \text { days }\end{array}$ & $\begin{array}{l}\text { Using a toothpaste with a } \\
\text { plaque-disclosing agent may } \\
\text { have reduced gingival inflamma- } \\
\text { tion slightly in the short term. }\end{array}$ & $\begin{array}{l}80 \\
(1 \text { observational } \\
\text { study) }\end{array}$ & $\begin{array}{l}\oplus \ominus \ominus \ominus \\
\text { Very lowa,b }\end{array}$ & $\begin{array}{l}\text { Study was a non ran- } \\
\text { domised cross over trial; } \\
\text { only } 2 \text { of the } 4 \text { arms were } \\
\text { relevant to this review. }\end{array}$ \\
\hline $\begin{array}{l}\text { Gingival inflammation medium term ( } 6 \text { weeks to } 12 \\
\text { months) }\end{array}$ & Not assessed & - & - & - \\
\hline $\begin{array}{l}\text { Plaque short term ( }<6 \text { weeks) } \\
\text { assessed with: Simplified Green \& Vermillion Plaque } \\
\text { Index (lower score means less plaque) } \\
\text { Follow-up: mean } 10 \text { days }\end{array}$ & $\begin{array}{l}\text { Using a toothpaste with a } \\
\text { plaque-disclosing agent may } \\
\text { have reduced plaque levels in the } \\
\text { short term. }\end{array}$ & $\begin{array}{l}80 \\
\text { (1 observational } \\
\text { study) }\end{array}$ & $\begin{array}{l}\oplus \ominus \ominus \ominus \\
\text { Very lowa,b }\end{array}$ & $\begin{array}{l}\text { Study was a non ran- } \\
\text { domised cross over trial; } \\
\text { only } 2 \text { of the } 4 \text { arms were } \\
\text { relevant to this review. }\end{array}$ \\
\hline Plaque medium term ( 6 weeks to 12 months) & Not assessed & - & - & - \\
\hline Quality of life & Not assessed & - & - & - \\
\hline Dental caries & Not assessed & - & - & - \\
\hline Adverse effects & None reported & - & - & - \\
\hline
\end{tabular}

ID: intellectual disability

\section{GRADE Working Group grades of evidence}

High certainty: we are very confident that the true effect lies close to that of the estimate of the effect.

Moderate certainty: we are moderately confident in the effect estimate: the true effect is likely to be close to the estimate of the effect, but there is a possibility that it is substantially different.

Low certainty: our confidence in the effect estimate is limited: the true effect may be substantially different from the estimate of the effect.

Very low certainty: we have very little confidence in the effect estimate: the true effect is likely to be substantially different from the estimate of effect.

aDowngraded three levels due to study design: single non-randomised study at unclear risk of bias, with no standard deviation reported.

bThe results of each arm in the cross-over trial were combined. 


\begin{tabular}{|c|c|c|c|c|}
\hline \multicolumn{5}{|c|}{ Individualised care plans compared to usual care for people with ID } \\
\hline \multicolumn{5}{|c|}{$\begin{array}{l}\text { Population: adults, aged } 22 \text { to } 57 \text { years with mixed levels of ID } \\
\text { Setting: residential } \\
\text { Intervention: individualised care plans based on a multidisciplinary assessment } \\
\text { Comparison: usual care - continued regular oral hygiene care, which was supported by a carer following institution's standard guidelines }\end{array}$} \\
\hline Outcomes & Impact & $\begin{array}{l}\text { Number of partici- } \\
\text { pants } \\
\text { (studies) }\end{array}$ & $\begin{array}{l}\text { Certainty of the evi- } \\
\text { dence } \\
\text { (GRADE) }\end{array}$ & Comment \\
\hline Gingival inflammation short term ( $<6$ weeks) & Not assessed & - & - & - \\
\hline $\begin{array}{l}\text { Gingival inflammation medium term ( } 6 \\
\text { weeks to } 12 \text { months) }\end{array}$ & Not assessed & - & - & - \\
\hline Plaque short term (< 6 weeks) & Not assessed & - & - & - \\
\hline $\begin{array}{l}\text { Plaque medium term ( } 6 \text { weeks to } 12 \text { months) } \\
\text { assessed with: subjective oral hygiene rating } \\
\text { and Improvement Differential } \\
\text { Follow-up: mean } 12 \text { months }\end{array}$ & $\begin{array}{l}\text { The use of individualised oral care plans } \\
\text { for people with ID may reduce plaque lev- } \\
\text { els over } 12 \text { months. The "improvement dif- } \\
\text { ferential" from baseline for the interven- } \\
\text { tion group was } 38 \% \text { compared to } 5 \% \text { for } \\
\text { the control group. }\end{array}$ & $\begin{array}{l}79 \\
\text { (1 observational } \\
\text { study) }\end{array}$ & $\begin{array}{l}\oplus \odot \odot \odot \\
\text { Very low }\end{array}$ & NRCT \\
\hline Quality of life & Not assessed & - & - & - \\
\hline Dental caries & Not assessed & - & - & - \\
\hline Adverse effects & None reported & - & - & - \\
\hline
\end{tabular}

\section{GRADE Working Group grades of evidence}

High certainty: we are very confident that the true effect lies close to that of the estimate of the effect.

Moderate certainty: we are moderately confident in the effect estimate: the true effect is likely to be close to the estimate of the effect, but there is a possibility that it is substantially different.

Low certainty: our confidence in the effect estimate is limited: the true effect may be substantially different from the estimate of the effect.

Very low certainty: we have very little confidence in the effect estimate: the true effect is likely to be substantially different from the estimate of effect. 


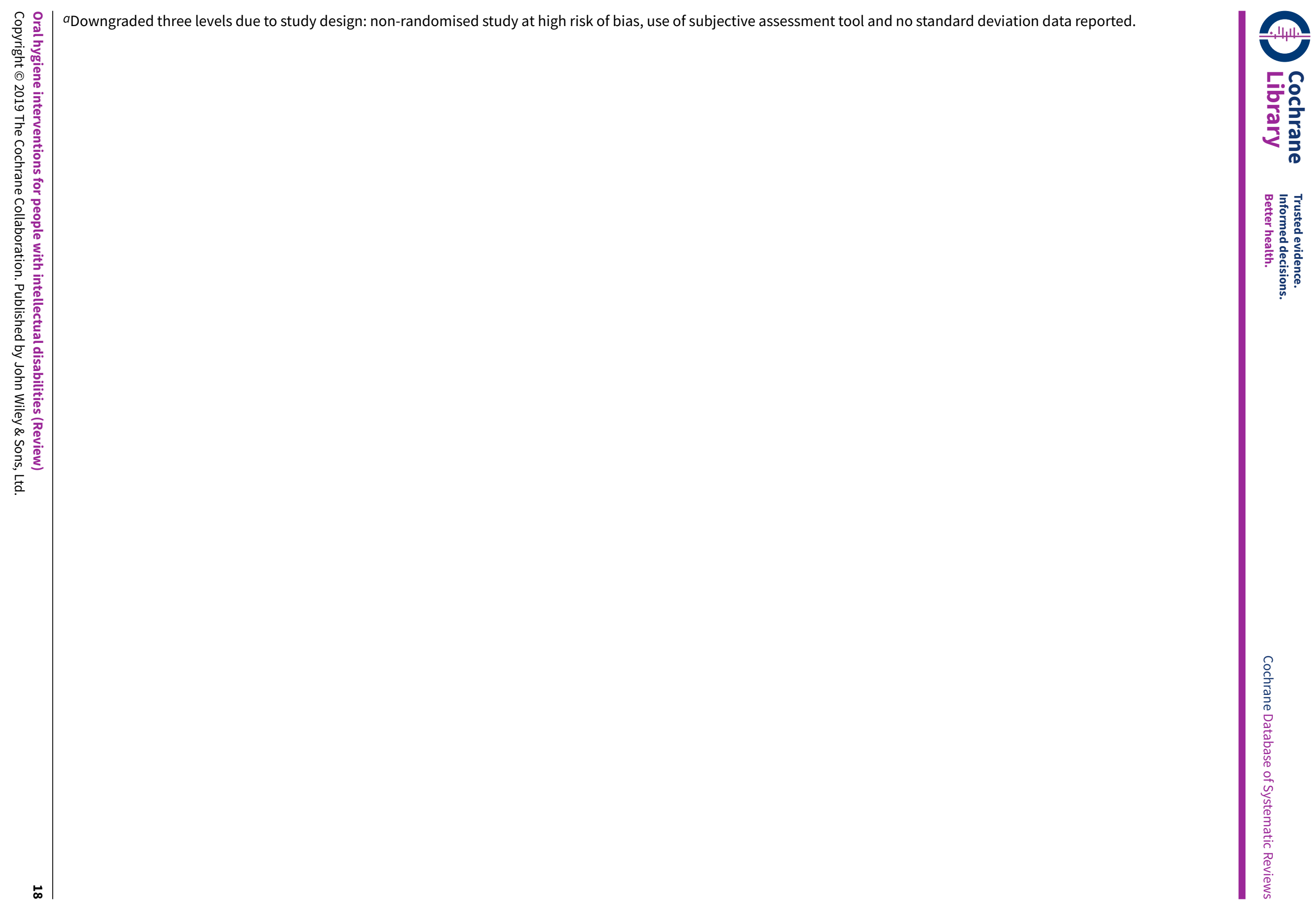




\section{B A C K G R O U N D}

\section{Description of the condition}

Oral hygiene is the process of cleaning the hard and soft tissues of the oral cavity (teeth, gums and tongue), fixed dental prostheses, oral appliances and dentures. Failure to perform regular and effective oral hygiene results in increased levels and varieties of the dental bacteria that make up dental plaque. Dental plaque has been shown to have a major role in the development of periodontal disease and dental caries, the two most common oral diseases (Axelsson 2004; Löe 2000)

Poor oral hygiene can affect a person's quality of life because of pain and discomfort when eating, poor self-esteem or sleep disturbance as a result of dental caries (Bonetti 2015). Evidence also exists linking levels of oral hygiene and periodontal status to diabetes, aspiration pneumonia and rheumatoid arthritis and evidence is emerging of a link with other systemic diseases and conditions such as cardiovascular disease, liver disease and obesity (Borgnakke 2015; Horwitz 2000).

People with an intellectual disability (ID) have a higher prevalence and greater severity of periodontal disease than the general population (Anders 2010; Campanaro 2014). The prevalence of dental caries in children with ID is similar to those of the same age in the general population (Whelton 2009). However, their oral health deteriorates at a faster rate as they move into adulthood. There are more untreated dental caries, more missing teeth and fewer restorations found in adults with ID than in the general population (Anders 2010; Catteau 2011; Crowley 2005; De Jongh 2008; Hennequin 2008; Morgan 2012; Oliveira 2013).

In the general population, brushing regularly with fluoridated toothpaste plays an important role in preventing dental caries and periodontal disease (Axelsson 2004; Broadbent 2011; Chestnutt 1998; Löe 1965; Löe 2000; Van der Weijden 2011; Zimmermann 2015). Whether this also applies to people with an ID is less clear, as many research studies on oral health exclude people with disabilities (Glassman 2009).

The removal of dental plaque may reduce the prevalence of periodontal disease and dental caries (Axelsson 1978; Axelsson 2004; Löe 1965; Löe 2000). However, the plaque must be removed thoroughly and regularly to achieve this effect. This health-related behaviour requires a person to have the relevant capabilities, opportunities and motivation to perform the necessary routines (Broadbent 2011; Stewart 1996; Watt 2005). For people with an ID, understanding the importance of removing dental plaque and developing the skills to do so requires more effort and achieving the standard of oral hygiene necessary for good oral health may require the support of others (Crowley 2005).

When considering the issues possibly influencing oral hygiene practices for the population with ID, it is important first to have a clear definition of ID; second, to consider the specific barriers to regular oral hygiene practices that might exist for people with ID; and third, to consider facilitators to promoting regular oral hygiene practices that might exist for people with ID.

\section{Definition of intellectual disability}

The most widely used current definition of disability comes from the World Health Organization's (WHO) International Classification of Functioning, Disability and Health (ICF), which incorporates the complex interactions between health conditions, environmental factors and personal factors. Regarding a person with an ID, this definition would consider how their personal factors, health condition and environment affect their lives (WHO 2001). Three elements are common for people with ID: a significant impairment of intelligence, a resultant significant reduction in adaptive behaviour/social functioning and the development of the condition (which persists throughout life) before the age of 18 years (Schalock 2010).

The WHO has also developed an operational definition of ID, which focuses mostly on the functional elements of ID, in the International Statistical Classification of Diseases and Related Health Problems, 10th version (ICD-10) (WHO 2011). The ICF definition of disability is designed to complement the ICD-10 definition.

The ICD-10 describes the four levels of ID as follows.

1. Mild ID (ICD-10 F70): intelligence quotient (IQ) range 50 to 69: "Ability to use speech in everyday situations; usually full independence in self care; Difficulties in identification of this population arise, as those with borderline disability may not be in contact with service providers."

2. Moderate ID (ICD-10 F71): IQ between 35 and 49: "Slow in comprehension; supervision of self care, retarded motor skills."

3. Severe ID (ICD-10 F72): IQ between 20 and 34: "Marked impairment of motor skills; clinically significant damage to Central Nervous System (CNS)."

4. Profound ID (ICD-10 F73): IQ less than 20: "Severely limited understanding; Immobility or restricted mobility; Incontinence; requires constant supervision; usually organic aetiology."

We acknowledge that other terms and definitions have been used to describe people with ID, both historically and currently. Whilst we acknowledge that the ICF is currently accepted as the most holistic definition of disability, we considered it unlikely to have been used in the studies that were potentially eligible for this review. Therefore, we planned to use the ICD-10 definition as the reference to determine the inclusion or exclusion of studies, as it provides a detailed nomenclature of diseases (Kurbasic 2008). In updates to this review, the ICF definition of ID will have become more commonly used in studies and will more accurately reflect the make-up of the population with disabilities. In addition, the ICF definitions are a better representation of populations requiring targeted services. However, the definition used (ICD-10) was considered by the team to accurately represent the population of interest in this review.

The heterogeneous nature of populations with ID adds further complexity to the assessment of interventions of relevance to these populations. An person's level of ID may impact their cognitive and physical abilities and personal and environmental factors, which may affect their response to an intervention. The response to an intervention therefore may be different for different people or groups of people with ID. We highlighted when studies provided details of the level of ID and personal and environmental factors of the participants. 


\section{Barriers to oral hygiene practice for people with intellectual} disability

Capability, opportunity and motivation are required to achieve adequate oral hygiene. Oral hygiene tasks are more difficult for people with ID; they may lack the manual dexterity or cognitive skills to carry out or understand the need for effective oral hygiene (Anders 2010; Löe 2000). There may be other associated conditions such as sensory impairment, anxiety, or chronic medical or behavioural conditions that militate against carrying out oral hygiene effectively (Gordon 1998; Minihan 2014; Prangnell 2008). In one 2003 Irish study, carers of people with ID identified some of the physical barriers caused by the disability such as an inability to rinse, difficulty getting a toothbrush into someone's mouth, an oversensitive mouth and a tendency to gag (Taylor-Dillon 2003). Nearly $10 \%$ of people with ID have dysphagia, which is associated with an increased tendency to choke or gag during toothbrushing (Chadwick 2009).

People with ID have chronic, poor oral hygiene and require more support with their oral hygiene. Carers for people with ID are often poorly trained (Crowley 2005). Oral hygiene is not seen as a priority for overstretched carers, particularly if it is not a personal priority (Kaye 2005; Rawlinson 2001). Lack of co-operation and challenging behaviour on the part of the person with ID may add to this burden.

\section{Facilitators for oral hygiene practice for people with intellectual disability}

Behaviour management techniques may improve the co-operation of people with ID when performing oral hygiene. Education may change carers' behaviour. Management techniques may improve the co-operation of people with ID when performing oral hygiene. Education may change carers' attitudes and improve their skills (Faulks 2000; Mac Giolla Phadraig 2013). Management support and guidance, stakeholder engagement, policy documents and increased resources may improve levels of oral hygiene provision in community and institutionalised settings (Glassman 2003; Glassman 2006; Glassman 2009). Specially designed or adapted oral hygiene aids may improve the oral hygiene skills of people with ID or their carers (Dougall 2008).

\section{Description of the intervention}

The interventions evaluated in this Cochrane Review focused on two distinct groups: people with an ID and the carers supporting, assisting or providing oral hygiene care for them. The range of carers involved was expected to be diverse; formal and nonformal personal carers with and without relevant training, with and without dental qualifications, and with and without psychology or related qualifications.

We defined oral hygiene in this review as the mechanical removal of plaque with manual or electric toothbrushes, interdental aids or other mechanical aids. Interventions designed to impact on levels of oral hygiene tend to categorise outcomes as clinical, such as reductions in plaque levels, periodontal diseases or dental caries, and behavioural/psychological, such as changes in comprehension, self-efficacy or motivation (Cooper 2013; Cooper 2014). This review included interventions that assessed clinical or behavioural/psychological outcomes or both. We considered any intervention that may have impacted on any combination of duration, frequency and effectiveness of oral hygiene practices.

\section{How the intervention might work}

Behavioural change models are relevant to oral hygiene behaviour and effective when used in interventions for people with ID (Renz 2007; Willems 2017). In one systematic review, Newton 2015 found using the constructs themselves to be a more reliable way of predicting behavioural change. Michie 2013 identified a taxonomy of 93 behavioural change techniques (BCTs) that provides a standardisation of the terminology used. In order to make the taxonomy of BCTs more accessible for clinicians, the BCTs can be grouped into 16 labelled clusters (Cane 2015; Michie 2011; Michie 2013). These labels provide a means to identifying the elements in an intervention that are likely to improve adherence and make routines easier to establish in relation to oral hygiene, even if they are not formally identified as BCTs. To further assist the analysis of interventions, Michie developed the 'Behavioural Change Wheel', which characterises the elements at three levels: a behaviour system at the hub, encircled by intervention functions and then by policy categories.

\section{The COM-B system}

We considered the interventions under the 'behaviour system' elements of Capability (which includes knowledge and skills), Opportunity (both physical and social) and Motivation (both automatic and reflexive), as outlined by Michie and colleagues as the COM-B system for understanding behaviour and behavioural change (Michie 2011).

Capability-based interventions, defined as the psychological and physical capacity to engage in the activity concerned, might be a knowledge-based intervention in relation to the comprehension and reasoning around why oral hygiene is required or a skills-based intervention that trains a person with ID in the physical skills of toothbrushing or that trains carers how to brush the teeth of a person with ID.

Opportunity-based interventions, which include both physical and social opportunities, should consider the elements beyond the individual, that is contexts such as the variety of settings in which the intervention is delivered or undertaken, for example, home, school, dental clinic, day care or residential care setting or the influences of the families or carers' attitudes regarding oral hygiene practices.

Motivation-based interventions, which include both automatic and reflective processes, might result in conscious decision-making where a goal or reward is identified to instigate or perpetuate a daily oral hygiene routine.

An intervention may change one or more of these behavioural elements resulting in the desired behaviour of performing regular oral hygiene.

\section{Why it is important to do this review}

The difficulties of providing dental care for people with disabilities have been acknowledged (Al Kindi 2016). Access to care is known to be more difficult; resources are stretched for parents, carers and healthcare professionals (Dougall 2008; Kaye 2005; Prabhu 2010; Sagheri 2013). The consequences of oral diseases for people with ID are more profound. Interventions designed to suit people with disabilities must show benefit (WHO 2011). Whilst an increasing number of good-quality systematic reviews of oral 
health interventions designed for the general population have been produced (Agnihotry 2016), Cochrane has not specifically reviewed the effectiveness of oral health interventions focused on people with disabilities (Cochrane Oral Health 2019).

One scoping review of interventions aimed at the wider population of people with disabilities has identified people with ID as being the most common target subpopulation (Waldron 2016). Oral hygiene status is one of the most common targets of oral health intervention for people with disabilities. This systematic review of oral hygiene interventions for people with ID allows us to draw conclusions about the effectiveness of these types of interventions. Furthermore, the review may increase dental professionals' understanding of behavioural change principles, inform the training of the dental team, and have an impact on the design and implementation of future oral health interventions for this population.

Cochrane Oral Health undertook an extensive prioritisation exercise in 2014 to identify a core portfolio of titles that were the most clinically important ones to maintain on the Cochrane Library (Worthington 2015). This exercise identified prevention and maintenance as priorities, which included oral hygiene adherence and caries prevention. Whilst not specifically identifying people with disabilities, the exercise also prioritised oral health promotion, specifically in institutionalised settings (Worthington 2015).

\section{OB JECTIVES}

To assess the effects (benefits and harms) of oral hygiene interventions, specifically the mechanical removal of plaque, for people with intellectual disabilities (ID).

\section{METHODS}

\section{Criteria for considering studies for this review}

\section{Types of studies}

We included randomised controlled trials (RCTs), including clusterRCTs, evaluating interventions to promote oral hygiene, specifically the mechanical removal of plaque, that were targeted at people with ID and groups such as parents, carers and other healthcare professionals caring for people with ID.

As we anticipated that the number of RCTs was likely to be low, we included some types of non-randomised studies (NRS): non-randomised controlled trials (NRCTs), controlled beforeafter studies, interrupted time series studies (ITS) and repeated measures studies.

ITS, where multiple data points are collected before and after the intervention and the intervention effect is measured against the preintervention trend and repeated measure studies, where these measures are taken on the same people at each time point, were only included if they had a clearly defined point in time when the intervention occurred and at least three data points before and three after the intervention.

We analysed RCTs and NRS separately, and presented RCT findings as the key evidence.

We applied no language restrictions.

\section{Types of participants}

People with an ID, living at home, in day care, in hospital or in a residential care setting. We considered all ID as defined by the ICD-10 (WHO 2011).

\section{Types of interventions}

We considered all oral hygiene interventions that used or promoted the mechanical removal of plaque. Interventions may have included, but were not limited to, the following components.

1. Oral hygiene-related knowledge-based interventions for participants or their carers, for example, education in the care of the teeth and gums, diseases of the teeth and gums, and prevention of diseases of the teeth and gums.

2. Oral hygiene-related behavioural interventions for participants or their carers that result in a behavioural change that can be observed and is replicable, for example, goal setting or positive reinforcement.

3. Oral hygiene-related skills training for participants, for example, interventions related to toothbrushing skills or other oral hygiene routines.

4. Oral hygiene-related skills training for carers, for example, interventions related to assessing toothbrushing skills or assisting participants with toothbrushing or other oral hygiene routines or providing oral hygiene care to participants.

To be eligible, controlled studies must have included a comparison with at least one of the following: usual care, no care or a similar alternative intervention. Examples of studies comparing similar alternative interventions might be a study comparing education provided in different formats, for example, face-to-face versus an information leaflet or a study comparing oral hygiene care provided after a training session versus oral hygiene care provided without training.

In relation to the definition of oral hygiene, we excluded interventions focusing on the chemical removal of plaque alone. The use of a chemical agent, which is used on a toothbrush and arguably removes plaque both mechanically and chemically, is complex, as the measurement of the resulting reduction of plaque cannot be assigned to either solely the mechanical or chemical action. We excluded these studies, except for those studies using a conventional non-prescription toothpaste normally used by the participants or using a non-conventional chemical agent for both the intervention and control in studies comparing the mechanical removal of plaque. For example, a study comparing a manual toothbrush with an electric toothbrush where both were used with a non-conventional toothpaste such as chlorhexidine or extrastrength fluoride toothpaste.

\section{Types of outcome measures}

Outcome measures included both behavioural and clinical measures.

We based the behavioural outcomes measured on the COM-B system and included indicators such as improved toothbrushing skills, changes in knowledge of participants and carers with regard to oral health, and increased episodes and duration of toothbrushing. We accepted any reasonable instrument used in the included studies such as questionnaires, interviews, observational outcomes, video recordings, diaries, self-reported outcomes or 
observational measures such as measuring toothpaste weight to check adherence with toothbrushing.

The clinical outcomes measured included indicators such as the prevalence/incidence of dental plaque, prevalence/incidence of periodontal disease (gum disease) and prevalence/incidence of dental caries. We used only published or validated assessments of the clinical outcomes or a comparable measure, for example, dental plaque measured using the Plaque Index, as outlined in Silness 1964 or Quigley-Hein Plaque Index as modified by Turesky and colleagues (Turesky 1970). Gingivitis measured using an index such as the Gingival Index, as outlined in Löe 1963 and Löe 1967 or the Modified Gingival Index (Lobene 1986). Dental caries was to be measured using an index such as the Decayed, Missing or Filled Teeth/Surface Index (dmft/s; DMFT/S) (Petersen 2013).

We considered all time frames for the delivery and follow-up of the intervention. The follow-up time frames were grouped for analysis into short, medium and long term, for all outcomes. A systematic review of the effects of oral hygiene on chronic periodontitis in the general population excluded studies lasting less than nine months, in order to differentiate between gingivitis and periodontitis (Hujoel 2005). The three RCTs included in that review were followed up for over three years. Mombelli 1998 reflected on the limitations of longitudinal studies of periodontal disease when considering the issues of measurement error in periodontal probing and the impact of concepts such as bursts of disease activity versus a continuous disease process, in relation to the progression of periodontal disease. Mombelli 1998 concluded that studies of 20 years or more in length would be required to achieve sufficient confidence in the results. One systematic review on the effects of toothbrushing frequency on the prevention of dental caries showed that the eligible interventions were all followed up for between 11 months and 15 years (Kumar 2016). The division of time frames was guided by a review of the literature on the effects of oral hygiene interventions on the oral health of people with ID and the knowledge, behaviour, attitude and self-efficacy of the people with ID or their carers.

1. Short-term follow-up (six weeks or less) may allow for some clinically visible signs of early changes in gingival health to become apparent, permitting the impact of possible differences in the microbial load, immune responses and environmental factors in this population group to manifest in clinically observable signs (Garmyn 1998). It may also be useful to observe the clinical changes resulting from an intervention without confounding the result with other variables, for example, the effect on oral hygiene following skills training without the variable of adherence to oral hygiene practices over time (Egelberg 1994). The early, clinically visible signs would include changes in plaque prevalence/incidence and changes in gingival bleeding or inflammation (Löe 1965).

2. Medium-term follow-up (six weeks to 12 months) may allow for some additional signs of changes in gingival health to be recorded compared to the baseline status, depending on the success of the intervention, such as gingival pocket probing depths, as well as consistent changes in knowledge and behaviour.

3. Long-term follow-up (greater than 12 months) would allow for some assessment of those measures that require a longer time frame to show change, such as dental caries prevalence/ incidence or clinical attachment loss or bone loss in relation to gingival disease, as well as continued follow-up on all other assessments. Long-term studies would also allow for followup on any short- or medium-term success regarding changes in oral hygiene behaviour that is crucial to maintaining oral health (Schou 1998). It must be accepted that any findings from these long-term interventions will be confounded by factors outside the study setting.

\section{Primary outcomes}

1. Gingival health of people with an ID, for example, plaque, gingival inflammation, calculus.

\section{Secondary outcomes}

1. Oral hygiene knowledge acquisition by people with an ID or the acquisition of knowledge by their carers with regard to oral hygiene.

2. Changes in behaviour, attitude and self-efficacy of people with an ID or their carers.

3. Quality of life changes or long-term, patient-centred outcomes for people with an ID (e.g. tooth retention).

4. Dental caries levels of people with an ID.

5. Any unintended (positive or negative) effects of the interventions.

Outcome measures relating to clinical outcomes must have been published or validated or comparable to measurements such as dental plaque levels (e.g. Plaque Score), gingival health (e.g. Gingival Index) or dental caries prevalence/incidence (i.e. DMFT/ S). Behavioural outcome assessments with regard to changes in capability, opportunity or motivation of the participant or carer for oral hygiene practices included any instrument used in the included studies that the review authors considered reasonable.

\section{Search methods for identification of studies}

\section{Electronic searches}

Cochrane Oral Health's Information Specialist conducted systematic searches in the following databases for randomised controlled trials and controlled clinical trials:

- Cochrane Oral Health's Trials Register (searched 4 February 2019);

- Cochrane Central Register of Controlled Trials (CENTRAL; in the Cochrane Register of Studies; searched 4 February 2019);

- MEDLINE Ovid (1946 to 4 February 2019);

- Embase Ovid (12 October 2016 to 4 February 2019);

- PsycINFO Ovid (1806 to 4 February 2019).

Details of the search strategies can be found in Appendix 1.

Subject strategies were modelled on the search strategy designed for MEDLINE Ovid. Due to the Cochrane Centralised Search Project to identify all clinical trials in the database and add them to CENTRAL, only the most recent months of the Embase database were searched. See the searching page on the Cochrane Oral Health website for more information. There were no other restrictions on the date of publication when searching the electronic databases; and searches were not limited by language or publication status. 


\section{Searching other resources}

The following trial registries were searched for ongoing studies to 4 February 2019 (see details of search terms used in Appendix 1):

1. US National Institutes of Health Ongoing Trials Register ClinicalTrials.gov (clinicaltrials.gov/);

2. WHO International Clinical Trials Registry Platform (apps.who.int/trialsearch).

We handsearched the specialist conference abstracts from the International Association of Disability and Oral Health (2006 to 2016). Conference abstracts prior to 2006 were not published and were not available from other sources.

We checked the bibliographies of included studies and any relevant systematic reviews identified for further references to relevant trials (Horsley 2011).

We considered any unpublished studies discovered using the search methods described above if they met the inclusion criteria for this review.

We considered adverse effects described in included studies only.

\section{Data collection and analysis}

\section{Selection of studies}

Five review authors independently examined the studies identified by the searches and ensured that each study was examined by at least two review authors, firstly by the titles and abstracts and then by obtaining and reading the full text, if the study was considered potentially relevant. We used specific inclusion criteria in our decision-making including the participants, interventions and study designs as outlined below. A sixth review author was the arbiter.

\section{Inclusion criteria:}

1. participants must have had an ID as defined by the ICD-10 or be a carer of a person with an ID;

2. the intervention must have related to oral hygiene;

3. study design must have been either an RCT or one of the NRS outlined earlier. Non-controlled studies had to have at least three before and three after measures;

4. complex interventions with an oral hygiene element were considered if the outcome from the oral hygiene component could be appraised separately.

Details such as keywords, authors, publication and institutions were available to the review authors. Any disagreements were discussed by the reviewing authors and the arbiter. We recorded justifications for all decisions. When we identified multiple reports of the same study, we linked these together as far as possible. We contacted study authors to clarify details regarding eligibility where necessary. We attempted to identify all relevant studies irrespective of language. Relevant non-English language papers were translated. Any studies identified by the searches, involving any of the review authors, were examined by other review authors.

\section{Data extraction and management}

Two review authors independently extracted data for each study using a specially designed data collection sheet, which was piloted by four of the review authors. We extracted data under the following headings: profile of the participants, number of participants, settings, details of interventions including details of the carers or personnel delivering or supporting the intervention and any BCTs identified within the interventions, outcomes measured, duration of intervention and follow-up, and study design. We designed a template for the Characteristics of included studies tables, based on the methods outlined in the protocol (Waldron 2017), and headings used in the data collection sheet, to highlight the important elements of the studies (Appendix 2).

\section{Assessment of risk of bias in included studies}

Two review authors independently assessed the risk of bias for each study, discussing any disagreements with the arbiter. We recorded justifications for decisions. To avoid any conflict of interest, we excluded team members who were authors of included studies from the 'Risk of bias' assessment of those studies and alternative review authors undertook this assessment.

We considered bias for RCTs and NRCT for the following domains: selection bias, performance bias, detection bias, attrition bias and reporting bias as per the Cochrane Handbook for Systematic Reviews of Interventions (Higgins 2011a). We considered bias for clusterRCTs under the two different units of measurement, the cluster and the participant, if provided and under the same domains as for RCTs (Campbell 2012).

We considered bias for non-controlled trials using the Effective Practice and Organisation of Care (EPOC) criteria for assessing risk of bias under the following headings (EPOC 2016; Sterne 2016).

1. Was the intervention independent of other changes?

2. Was the shape of the intervention effect prespecified?

3. Was the intervention unlikely to affect data collection?

4. Was knowledge of the allocated interventions adequately prevented during the study?

5. Were incomplete outcome data adequately addressed?

6 . Was the study free from selective outcome reporting?

We supplemented this with information on participant and study characteristics where appropriate.

We completed a 'Risk of bias' table for each included study and rated each study as high risk, low risk or unclear risk of bias for the separate domains and presented the results graphically (Figure 1; Figure 2). All domains were regarded as equally important in this review. The overall risk of bias within a study was determined across domains depending on the number of low, unclear and high ratings: if there was at least one rating of high risk of bias for the study, it was rated overall as high risk of bias; if all ratings were low risk of bias, the study was rated low overall; if there was a mix of low and unclear ratings, the study was rated overall as being at unclear risk of bias. 
Figure 1. Risk of bias graph: review authors' judgements about each risk of bias item presented as percentages across all included studies.

\begin{tabular}{|c|c|c|c|c|c|}
\hline \multicolumn{6}{|c|}{ Random sequence generation (selection bias) } \\
\hline \multicolumn{6}{|c|}{ Allocation concealment (selection bias) } \\
\hline \multicolumn{6}{|c|}{ Blinding of participants and personnel (performance bias) } \\
\hline \multicolumn{6}{|c|}{ Blinding of outcome assessment (detection bias) } \\
\hline \multicolumn{6}{|c|}{ Incomplete outcome data (attrition bias) } \\
\hline \multicolumn{6}{|c|}{ Selective reporting (reporting bias) } \\
\hline \multicolumn{6}{|c|}{ Was the intervention independent of other changes? (ITS) } \\
\hline \multicolumn{6}{|c|}{ Was the shape of the intervention effect prespecified? (ITS) } \\
\hline \multicolumn{6}{|c|}{ Was the intervention unlikely to affect data collection? (ITS) } \\
\hline \multicolumn{6}{|c|}{ Was knowledge of the allocated interventions adequately prevented during the study? (ITS) } \\
\hline \multicolumn{6}{|c|}{ Were incomplete outcome data adequately addressed? (ITS) } \\
\hline \multicolumn{6}{|c|}{ Was the study free from selective outcome reporting? (ITS) } \\
\hline & & $25 \%$ & $50 \%$ & $75 \%$ & $100 \%$ \\
\hline Low risk of bias & $\square$ Unclear risk of bias & High risk o & & & \\
\hline
\end{tabular}


Figure 2. Risk of bias summary: review authors' judgements about each risk of bias item for each included study.

\begin{tabular}{|c|c|c|c|c|c|c|c|c|c|c|c|c|}
\hline & 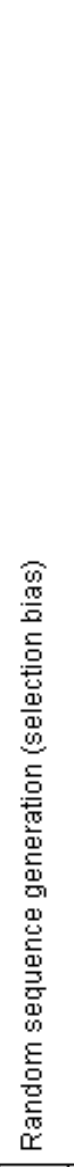 & 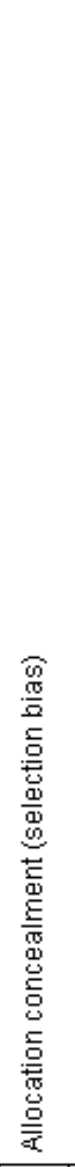 & 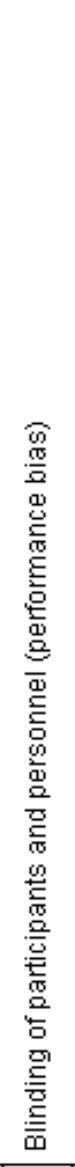 & 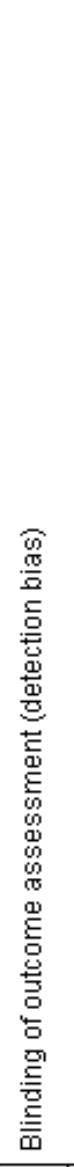 & 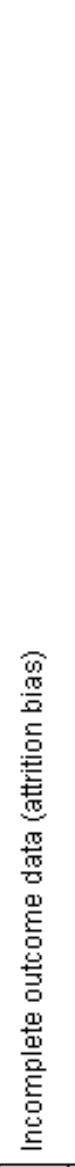 & 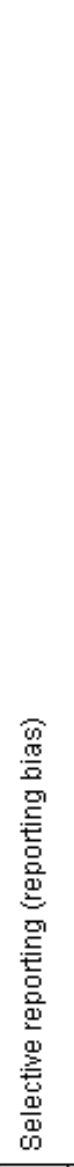 & 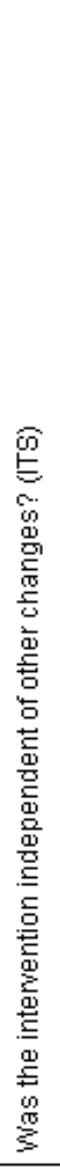 & 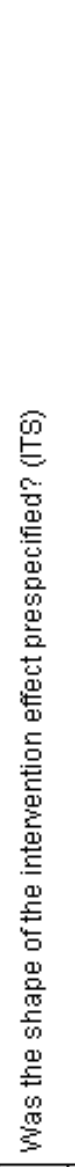 & 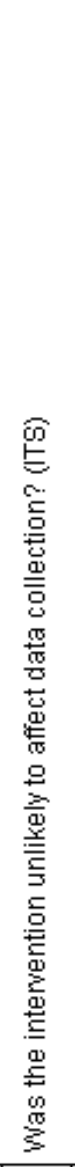 & 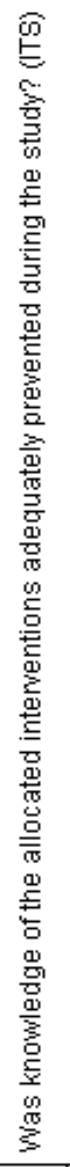 & 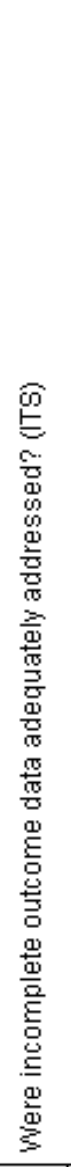 & 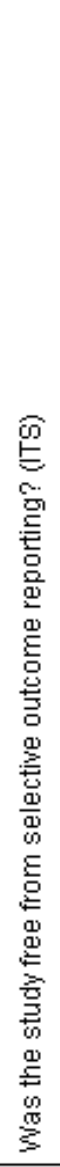 \\
\hline Abramson 1972 & & & & & & & $?$ & $\odot$ & + & $\odot$ & $\odot$ & + \\
\hline Albino 1979 & + & $?$ & $?$ & 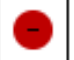 & $\odot$ & $\odot$ & & & & & & \\
\hline Altabet 2003 & 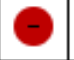 & $O$ & + & + & + & $?$ & & & & & & \\
\hline Bickley 1990 & + & $?$ & $?$ & + & $\odot$ & - & & & & & & \\
\hline Bildt 2010 & $\odot$ & + & + & + & + & $?$ & & & & & & \\
\hline Bouter 1979 & & & & & & & $?$ & + & + & $\odot$ & $\odot$ & $?$ \\
\hline Bratel 1991 & $?$ & $\Theta$ & $?$ & $?$ & $\odot$ & $?$ & & & & & & \\
\hline Carr 1997 & $?$ & $?$ & $?$ & $?$ & $?$ & $?$ & & & & & & \\
\hline Christen 2007 & $\odot$ & $\odot$ & $\odot$ & $\odot$ & $\odot$ & $?$ & & & & & & \\
\hline Dôgan 2004 & $?$ & $?$ & $\theta$ & $?$ & $?$ & $?$ & & & & & & \\
\hline Ferozali 2007 & $\odot$ & $\odot$ & $?$ & $\odot$ & $\odot$ & $?$ & & & & & & \\
\hline Garcia-Carrillo 2016 & + & + & $?$ & + & + & $?$ & & & & & & \\
\hline Gertenrich $1967 \mathrm{a}$ & $\odot$ & $?$ & $?$ & $\odot$ & $\odot$ & $?$ & & & & & & \\
\hline Gertenrich $1967 \mathrm{~b}$ & $\odot$ & $?$ & $?$ & + & $?$ & $?$ & & & & & & \\
\hline
\end{tabular}


Figure 2. (Continued)

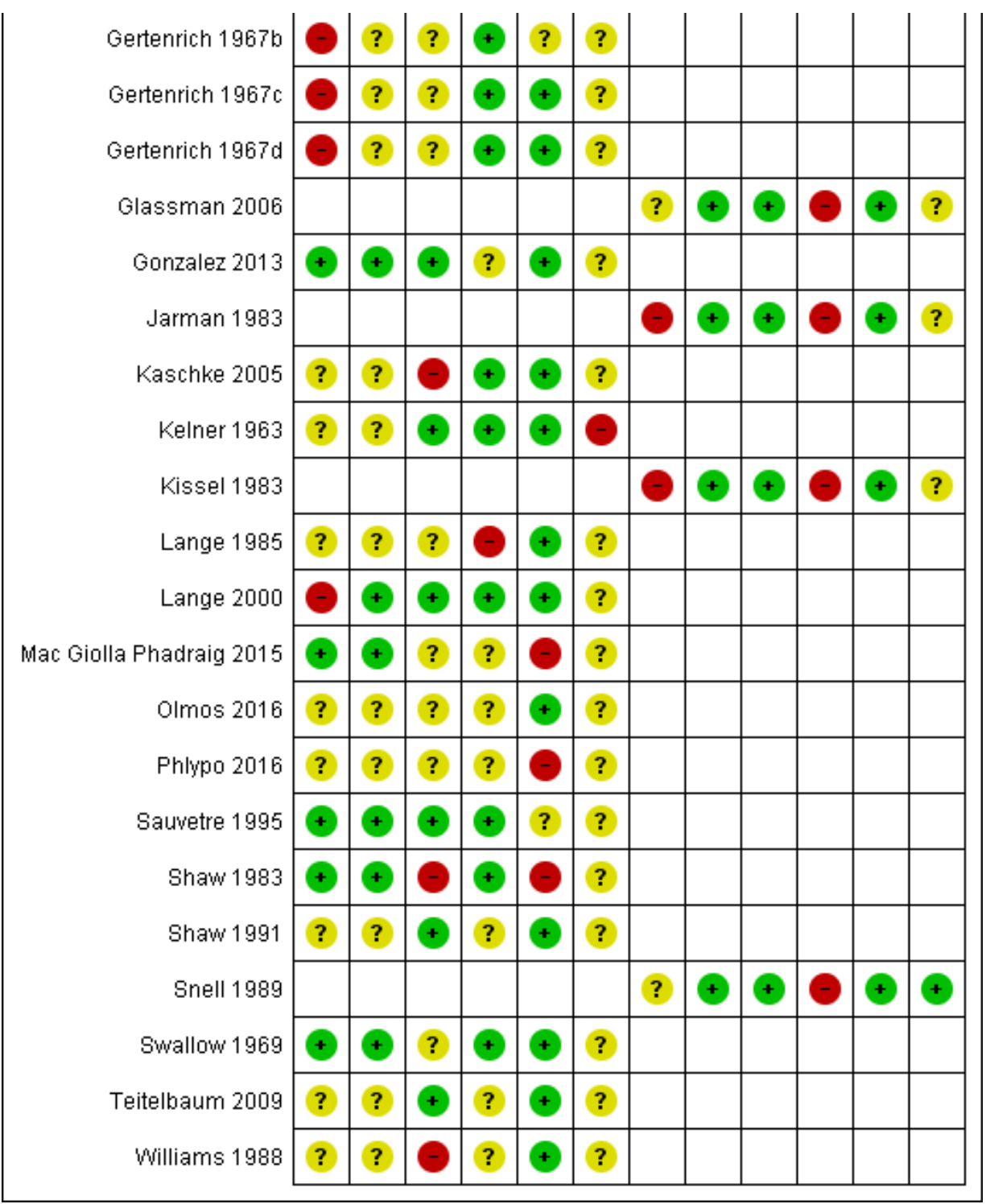

\section{Measures of treatment effect}

For continuous data (e.g. plaque levels, self-efficacy scale), we used the mean difference (MD) when the same scale was used or standardised mean difference (SMD) if more than one scale was used to measure the same outcome, along with the $95 \%$ confidence interval $(\mathrm{Cl})$. Had we found dichotomous data, we would have used risk ratios (RR) and $95 \% \mathrm{Cl}$.

\section{Unit of analysis issues}

We considered the unit of analysis with regard to whether the intervention was targeted at individual or group level, whether there was more than one element to the intervention and whether there were different time points measured in the intervention. When possible, we planned to adjust for clustering if participants were allocated to the same intervention in groups (e.g. family members or care homes), for example, in cluster-RCTs. To account for the effect of clustering, we planned to estimate inflated standard errors (SEs) (Deeks 2011).
With regard to RCTs and other comparative studies, we considered adjustments to avoid multiple use of participants in multiarm or cross-over studies. If an intervention arm was included in more than one treatment comparison, we divided the number of participants in that arm and the number of events by the total number of treatment comparisons. This approach retained original information but reduced the precision of the pooled estimates.

We combined multiple arms when appropriate, for example, participants who brushed their own teeth and participants who required assistance or had their teeth brushed for them, or participants with different levels of disabilities. Findings for the separate arms are reported in the additional tables, but were pooled for the data analysis.

With regard to cross-over trials, data from these need to be reported in a specific way to allow their inclusion in meta-analyses (e.g. showing the data from each treatment period to allow calculation of the SE of the MD or data to approximate the correlation between 
results from different treatment periods). However, the necessary data were not available from the cross-over trials that we identified.

Where multiple time points were presented in the studies, we selected the time point for each study that could be compared to another study in each of the time periods (short, medium or long term) or the longest time point in each time period or both.

\section{Dealing with missing data}

We made all reasonable efforts to contact authors for details and reasons for any missing outcome data (Young 2011). If data were missing and no information was available in the paper or was not obtainable by contacting the authors, we planned to assume, for dichotomous data, that dropout was due to the intervention being unsuccessful. No dichotomous data were reported in this review. We planned to perform sensitivity analyses to assess the effects of these assumptions, for example, missing data for intervention studies could have been dealt with by using intention-to-treat numbers. For continuous data, we planned to use this information if we were able to calculate the data from other data provided, for example, calculating standard deviation (SD) from SE and P values (Higgins 2011b).

\section{Assessment of heterogeneity}

We planned to assess levels of statistical heterogeneity by observing the $\mathrm{Cl}$ within studies and, to a lesser extent, statistically by use of the $\mathrm{Chi}^{2}$ test to determine whether observed differences in results were compatible with chance alone. Interpretation of the $\mathrm{Chi}^{2}$ would take into consideration the sample size and number of studies included in the meta-analysis.

We planned to quantify heterogeneity with the $I^{2}$ statistic, using the following guide. Some level of heterogeneity was expected (Higgins 2011a).

1. $0 \%$ to $40 \%$ : might not be important.

2. $30 \%$ to $60 \%$ : may represent moderate heterogeneity.

3. $50 \%$ to $90 \%$ : may represent substantial heterogeneity.

4. $75 \%$ to $100 \%$ : very substantial ('considerable') heterogeneity.

\section{Assessment of reporting biases}

Thorough searching of multiple databases should limit general reporting biases. We kept reporting biases due to such things as time lag bias (Hopewell 2007), selective outcome reporting (Dwan 2014), and publication bias (Hopewell 2009), to a minimum by inclusion of prospective trial registers; had we identified at least 10 studies in a comparison, we would have created funnel plots to explore publication bias and other biases related to study size. We made every effort to contact authors for results of outcomes included in their study objectives but not reported.

\section{Data synthesis}

We undertook meta-analyses only when studies were sufficiently homogeneous in their participants, interventions and outcomes; otherwise, we presented a narrative of individual study findings. We planned to combine RRs for dichotomous data and MDs or SMDs for continuous data, using random-effects models if at least four studies were included in a meta-analysis. We used fixedeffect models when there were fewer than four studies and when heterogeneity was reasonably low.

\section{Subgroup analysis and investigation of heterogeneity}

We planned to consider subgroup analysis for topics such as the following if sufficient data were available (Bender 2008).

1. Level of ID.

2. With versus without support of dental professionals.

3. Non-formal carers (i.e. parents or siblings) versus formal carers.

4. Setting of the intervention (i.e. home-based versus residential/ institutional/school-based interventions).

5. BCT used based on the COM-B system.

6. Short-term interventions versus long-term interventions.

For this version of the review, the amount of data was too small to undertake any purposeful analysis.

\section{Sensitivity analysis}

We had planned to undertake sensitivity analyses based on risk of bias; however, there were insufficient studies for a sensitivity analysis of this type. We undertook post hoc analysis excluding two studies that included some participants with an IQ above 70 (Garcia-Carrillo 2016; Gertenrich 1967a); no relevant effect was noticed.

\section{Summarising findings and assessing the certainty of the evidence}

We created 'Summary of findings' tables for the nine comparisons identified. We summarised the findings for the main outcomes measured: gingival inflammation or bleeding, and plaque (short, medium and long term); quality of life; dental caries; adverse effects. For training interventions, we also included oral health knowledge, and behaviour, attitude and self-efficacy. We used GRADEpro software to assess the certainty of the evidence under the following headings: study design (risk of bias); inconsistency; indirectness; imprecision; publication bias; large effect; plausible confounding; dose response gradient (Schünemann 2013). We categorised the certainty of the body of evidence for each of the main outcomes for each comparison as high, moderate, low or very low.

\section{RES U L T S}

\section{Description of studies}

\section{Results of the search}

The searches from the databases resulted in 3805 records and an additional 41 records were identified from other sources. After removal of duplicates, review authors screened 3020 records by title and abstract using Covidence software (Covidence 2018), of which 2794 were judged to be irrelevant and rejected. Following this process, we attempted to source 226 full-text records for assessment for eligibility against the inclusion and exclusion criteria outlined in the protocol (Waldron 2017). We rejected 153 records. We excluded 23 records ( 22 studies) for reasons listed in the Characteristics of excluded studies table; three studies are awaiting classification (see Characteristics of studies awaiting classification table), and one is ongoing (see Characteristics of ongoing studies table). Therefore, 34 studies (46 records) met the inclusion criteria for this review (Figure 3). 
Figure 3. Study flow diagram. ID: intellectual disability; NRS: non-randomised study; RCT: randomised controlled trial.

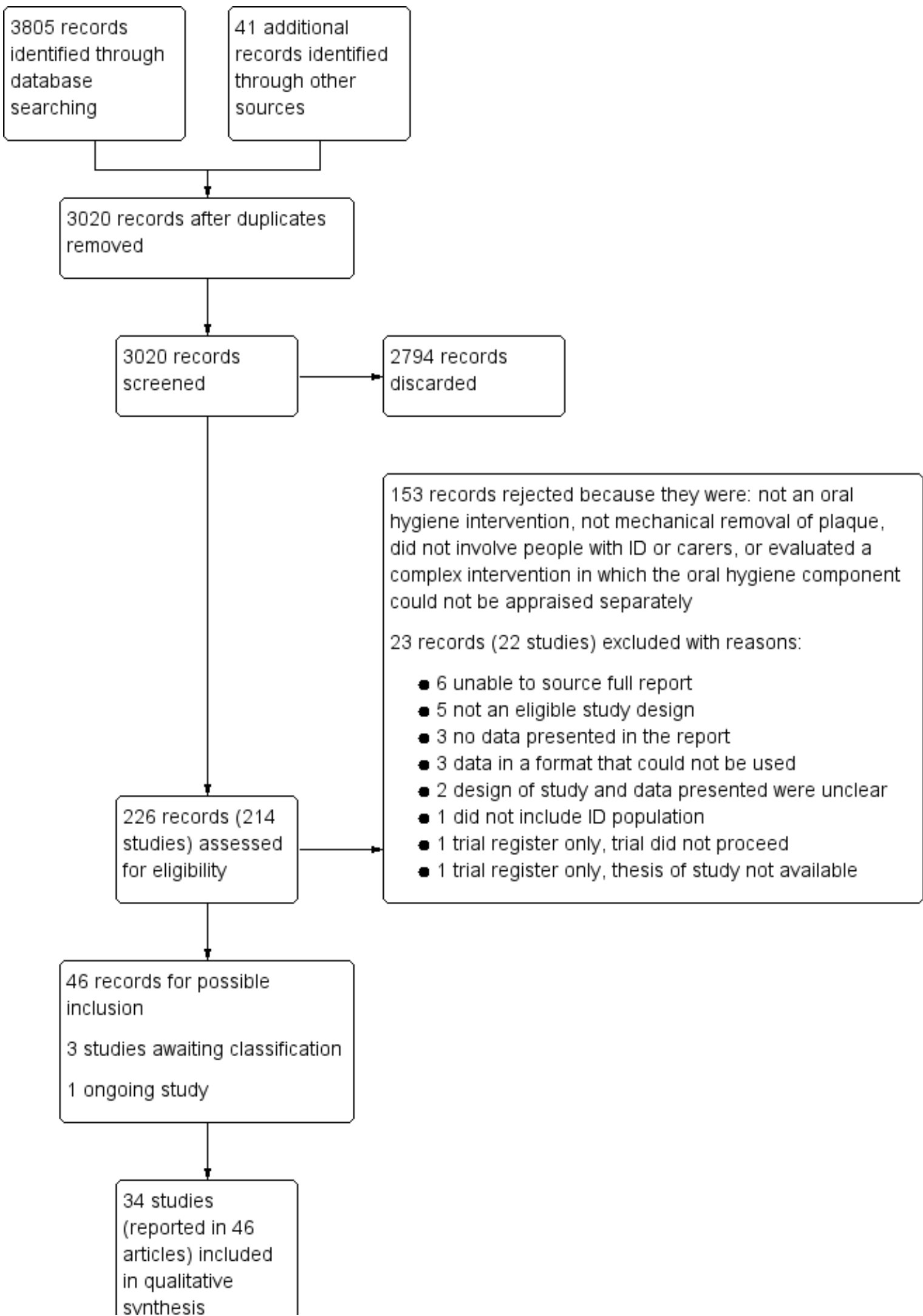


Figure 3. (Continued)

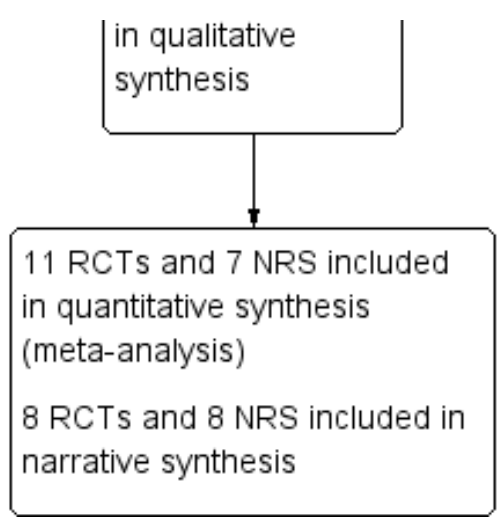

Three of the review authors piloted data extraction on a small number of records and some clarifications to the criteria were made following a team discussion (see the details in the Differences between protocol and review section). At least two review authors independently undertook screening and extraction: three review authors were involved in the screening of titles and abstracts (CW, $\mathrm{JN}, \mathrm{CMGP}$ ); five review authors were involved in the extraction process (CW, JN, CMGP, SG, CC).

\section{Included studies}

The study details are summarised in the Characteristics of included studies table.

\section{Characteristics of the methodologies}

\section{Design}

Thirty-four studies met the inclusion criteria for this review: 19 RCTs and 15 NRSs. RCTs and NRSs are reported separately.

Fifteen studies were parallel-group RCTs (Albino 1979; Bickley 1990; Bildt 2010; Christen 2007; Dôgan 2004; Ferozali 2007; Gonzalez 2013; Kaschke 2005; Kelner 1963; Lange 1985; Olmos 2016; Phlypo 2016; Sauvetre 1995; Shaw 1983; Swallow 1969), and four were cluster RCTs (Carr 1997; Garcia-Carrillo 2016; Mac Giolla Phadraig 2015; Shaw 1991). Four RCTs were cross-over trials: one trial had two arms (Shaw 1983), and the others had three arms (Christen 2007; Dôgan 2004; Kaschke 2005).

Of the NRS, nine studies were NRCTs (Altabet 2003; Bratel 1991; Gertenrich 1967a; Gertenrich 1967b; Gertenrich 1967c; Gertenrich 1967d; Lange 2000; Teitelbaum 2009; Williams 1988), three were ITS studies (Glassman 2006; Jarman 1983; Kissel 1983), and three were repeat measure (RM) studies (Abramson 1972; Bouter 1979; Snell 1989).

\section{Date and duration}

The 19 RCTs were reported between 1963 and 2016. One RCT included long-term follow-up (24 months) (Shaw 1991); eight included medium-term follow-up (greater than six weeks to less than 12 months) (Bickley 1990; Bildt 2010; Carr 1997; Ferozali 2007; Garcia-Carrillo 2016; Kelner 1963; Mac Giolla Phadraig 2015; Phlypo 2016); and 10 had only short-term follow-up (six weeks or less) (Albino 1979; Christen 2007; Dôgan 2004; Gonzalez 2013; Kaschke 2005; Lange 1985; Olmos 2016; Sauvetre 1995; Shaw 1983; Swallow 1969).
The 15 NRS were reported between 1967 and 2009. Two NRS had long-term follow-up (16 to 19 months) (Bratel 1991; Snell 1989), 10 had medium-term follow-up (Altabet 2003; Bouter 1979; Gertenrich 1967a; Gertenrich 1967b; Gertenrich 1967c; Gertenrich 1967d; Glassman 2006; Jarman 1983; Kissel 1983; Williams 1988), and two had only short-term follow-up (Lange 2000; Teitelbaum 2009). The duration was unclear for one NRS (Abramson 1972).

\section{Size}

The number of participants in the studies was based on the number of participants recorded at baseline; some studies reported the intention-to-treat numbers.

The number of participants in the RCTs ranged from 18 to 329. Ten RCTs had fewer than 50 participants (Albino 1979; Bickley 1990; Bildt 2010; Christen 2007; Dôgan 2004; Ferozali 2007; Gonzalez 2013; Kaschke 2005; Lange 1985; Sauvetre 1995), five RCTs had between 50 and 100 participants (Carr 1997; Garcia-Carrillo 2016; Phlypo 2016; Shaw 1983; Swallow 1969), and four RCTs had more than 100 participants (Kelner 1963; Mac Giolla Phadraig 2015; Olmos 2016; Shaw 1991). Most studies evaluated interventions aimed specifically at people with ID. Four of the RCTs included carers as participants: one assessed outcomes for the carers only (Gonzalez 2013), and three assessed outcomes for both people with ID and carers (Mac Giolla Phadraig 2015; Olmos 2016; Phlypo 2016). One did not report the number of carers involved (Olmos 2016).

The number of participants in the NRS ranged from three to 79 . Four NRS had fewer than 20 participants (Abramson 1972; Bouter 1979; Kissel 1983; Snell 1989), eight NRS had between 20 and 50 participants (Bratel 1991; Gertenrich 1967a; Gertenrich 1967c; Gertenrich 1967d; Glassman 2006; Jarman 1983; Teitelbaum 2009; Williams 1988), and three NRS had more than 50 participants (Altabet 2003; Gertenrich 1967b; Lange 2000). Two NRS assessed outcomes for both people with ID and carers (Glassman 2006; Kissel 1983); one NRS involved carers in the study but only assessed outcomes for the people with ID (Lange 2000).

\section{Location, language and setting}

The studies were carried out in 10 different countries: Belgium (two RCTs: Phlypo 2016; Sauvetre 1995), Brazil (one NRS: Teitelbaum 2009), Germany (three RCTs: Christen 2007; Kaschke 2005; Olmos 2016), Ireland (one RCT: Mac Giolla Phadraig 2015), Spain (one RCT: Garcia-Carrillo 2016), Sweden (one NRS: Bratel 1991), the Netherlands (one RCT: Bildt 2010; one NRS: Bouter 1979), Turkey 
(one RCT: Dôgan 2004), the UK (four RCTs: Bickley 1990; Shaw 1983; Shaw 1991; Swallow 1969), and the USA (six RCTs: Albino 1979; Carr 1997; Ferozali 2007; Gonzalez 2013; Lange 1985; Kelner 1963; 12 NRS: Abramson 1972; Altabet 2003; Gertenrich 1967a; Gertenrich 1967b; Gertenrich 1967c; Gertenrich 1967d; Glassman 2006; Jarman 1983; Kissel 1983; Lange 2000; Snell 1989; Williams 1988). Three studies were written in German (Christen 2007; Kaschke 2005; Olmos 2016), and one in Dutch (Bildt 2010).

The settings were residential (eight RCTs: Carr 1997; Ferozali 2007; Gonzalez 2013; Lange 1985; Mac Giolla Phadraig 2015; Olmos 2016; Phlypo 2016; Swallow 1969; 13 NRSs: Abramson 1972; Altabet 2003; Bouter 1979; Gertenrich 1967a; Gertenrich 1967b; Gertenrich 1967c; Gertenrich 1967d; Glassman 2006; Jarman 1983; Kissel 1983; Lange 2000; Teitelbaum 2009; Williams 1988); school (four RCTs: Albino 1979; Dôgan 2004; Garcia-Carrillo 2016; Shaw 1983; one NRS: Snell 1989), day centre (three RCTs: Bickley 1990; Sauvetre 1995; Shaw 1991), or mixed (two RCTs: Christen 2007; Kelner 1963; one NRS (Bratel 1991). Two RCTs were unclear (Bildt 2010; Kaschke 2005).

\section{Ethical approval and consent}

Twenty-one studies did not report having ethical approval; 11 RCTs (Bildt 2010; Christen 2007; Dôgan 2004; Ferozali 2007; GarciaCarrillo 2016; Gonzalez 2013; Kaschke 2005; Lange 1985; Mac Giolla Phadraig 2015; Phlypo 2016; Sauvetre 1995), and one NRS (Teitelbaum 2009) reported having received some form of ethical approval; the one quasi-RCT was unclear on the issue (Lange 1985).

The participants or guardians gave consent in 14 RCTs (Bickley 1990; Carr 1997; Christen 2007; Dôgan 2004; Ferozali 2007; GarciaCarrillo 2016; Gonzalez 2013; Kelner 1963; Lange 1985; Mac Giolla Phadraig 2015; Phlypo 2016; Shaw 1983; Shaw 1991; Sauvetre 1995), and three of the NRS (Glassman 2006; Lange 2000; Teitelbaum 2009); four RCTs and 11 NRS did not report consent; one RCT (Bildt 2010) and one NRS (Kissel 1983) were unclear.

\section{Characteristics of the participants}

In relation to people with ID, the studies included 1795 participants at baseline. In some studies, the difference between intention-totreat numbers and those actually included at baseline was not explained. A total of 92 participants were reported as lost to followup; however, this number should be treated with caution as it is possible that those participants included at baseline were the more compliant participants and other potential participants were lost before baseline data could be collected. There were 17 studies that actively involved the carers of the people with ID in the delivery of the intervention, of these, seven focused on the carers as participants: six provided details of the number of carers (three RCTs: Gonzalez 2013; Mac Giolla Phadraig 2015; Phlypo 2016; three NRS: Glassman 2006; Kissel 1983; Lange 2000). A total of 354 carers provided baseline data; 92 were lost to follow-up.

Twenty-six studies had both males and females; one study had only males (Abramson 1972); seven studies did not report the sex of participants (Bickley 1990; Dôgan 2004; Kaschke 2005; Kelner 1963; Phlypo 2016; Sauvetre 1995; Shaw 1983).

Two RCTs (Dôgan 2004; Shaw 1983) and six NRS (Abramson 1972; Gertenrich 1967c; Gertenrich 1967d; Kissel 1983; Snell 1989; Teitelbaum 2009) involved only participants with ID who were less than 18 years old. Seven studies recruited both children and adults (Albino 1979; Gertenrich 1967a; Jarman 1983; Kelner 1963; Lange 1985; Swallow 1969; Williams 1988). Seventeen studies had participants who were aged 18 years or older, with an age range of 18 to 79 years. Bildt 2010 reported only mean age and three studies did not report the age profile (Bickley 1990; Gonzalez 2013; Phlypo 2016).

We converted study descriptions of the participants' level of ID to the ICD-10 descriptors: mild, moderate, severe and profound.

\begin{tabular}{llll}
\hline Interventions $\quad$ Design & ICD-10 category
\end{tabular}

1. Special toothbrush RCT Dôgan 2004: mild Christen 2007

Bildt 2010: moderate to profound Kaschke 2005

Ferozali 2007: profound $\quad$ Sauvetre 1995

\begin{tabular}{|c|c|c|c|}
\hline & NRS & Williams 1988: profound & - \\
\hline 2. Electric toothbrush & $\mathrm{RCT}$ & Dôgan 2004: mild & Carr 1997 \\
\hline
\end{tabular}

$\begin{array}{llll}\text { 2. Electric toothbrush } & \text { RCT } & \text { Dôgan 2004: mild } & \text { Carr } 1997\end{array}$

Garcia-Carrillo 2016: mild to moderate; and some partici- Christen 2007 pant IQs $>70$

Shaw 1983: severe 
Gertenrich 1967b; Gertenrich 1967c: severe to profound

\begin{tabular}{|c|c|c|c|}
\hline \multirow[t]{2}{*}{ 3. Training of carers } & $\mathrm{RCT}$ & $\begin{array}{l}\text { Mac Giolla Phadraig 2015: mild to profound } \\
\text { Phlypo 2016: severe to profound }\end{array}$ & $\begin{array}{l}\text { Gonzalez } 2013 \\
\text { Olmos } 2016\end{array}$ \\
\hline & NRS & $\begin{array}{l}\text { Glassman 2006: mild to severe } \\
\text { Lange 2000: moderate to profound } \\
\text { Kissel 1983: severe to profound }\end{array}$ & - \\
\hline \multirow[t]{2}{*}{$\begin{array}{l}\text { 4. Training of people } \\
\text { with ID }\end{array}$} & $\mathrm{RCT}$ & $\begin{array}{l}\text { Albino 1979: moderate to severe } \\
\text { Lange 1985: mild to moderate }\end{array}$ & - \\
\hline & NRS & $\begin{array}{l}\text { Bouter 1979: moderate } \\
\text { Abramson 1972: severe } \\
\text { Jarman 1983: severe } \\
\text { Snell 1989: severe to profound }\end{array}$ & - \\
\hline \multirow{2}{*}{$\begin{array}{l}\text { 5. Scheduled dental } \\
\text { recall intervals and su- } \\
\text { pervised toothbrush- } \\
\text { ing }\end{array}$} & $\mathrm{RCT}$ & - & Shaw 1991 \\
\hline & NRS & - & - \\
\hline \multirow{2}{*}{$\begin{array}{l}\text { 6. Clinical pho- } \\
\text { tographs }\end{array}$} & $\mathrm{RCT}$ & - & Bickley 1990 \\
\hline & NRS & - & - \\
\hline \multirow{2}{*}{$\begin{array}{l}\text { 7. Varying frequency } \\
\text { of toothbrushing }\end{array}$} & $\mathrm{RCT}$ & - & Swallow 1969 \\
\hline & NRS & - & - \\
\hline \multirow{2}{*}{$\begin{array}{l}\text { 8. Plaque-disclosing } \\
\text { agent }\end{array}$} & $\mathrm{RCT}$ & - & - \\
\hline & NRS & Teitelbaum 2009: mild to moderate & - \\
\hline \multirow{2}{*}{$\begin{array}{l}\text { 9. Individualised care } \\
\text { plan }\end{array}$} & $\mathrm{RCT}$ & - & - \\
\hline & NRS & Altabet 2003: mild to profound & - \\
\hline
\end{tabular}

ICD-10: International Statistical Classification of Diseases and Related Health Problems, 10th revision; ID: intellectual disability; IQ: intelligence quotient; NRS: non-randomised study; RCT: randomised controlled trial.

Two studies reported including some participants with an IQ above 70 (ranging as high as 87 (Garcia-Carrillo 2016) and 94 (Gertenrich 1967a)). We undertook a sensitivity analysis to determine the effect on the effect size of the outcomes of excluding these studies. There was no relevant effect.

Fifteen studies reported that participants with ID had comorbidities: nine RCTs (Albino 1979; Bildt 2010; Carr 1997; Christen 2007; Ferozali 2007; Garcia-Carrillo 2016; Kaschke 2005; Shaw 1983; Shaw 1991) and six NRS (Gertenrich 1967a; Gertenrich 1967b; Gertenrich 1967c; Glassman 2006; Jarman 1983; Snell 1989). The most frequent conditions reported were cerebral palsy (five studies), epilepsy (four studies), Down's syndrome (three studies), visual impairment (three studies) and hearing impairment (two studies). Other conditions included; anxiety disorder, asthma, autism, chronic bronchitis, depression, diabetes, emesis, enteral feeding, gastro-oesophageal reflux disease, heart defect, hepatitis C, hiatal hernia, Hunter's syndrome, hydrocephaly, hypertension, hypothyroidism, muscular dystrophy, ocular tension, paranoid schizophrenia, phenylketonuria disorder, Prader-Willi syndrome, speech difficulties, swallowing problems, Tay-Sachs disorder, thyroid problems, Turner's syndrome, along with general terms to describe physical disabilities. One study reported that participants 
were excluded if they had any systemic disease (Teitelbaum 2009), and 18 studies did not report any comorbidities.

\section{Characteristics of the interventions}

More than one study evaluated each of the following comparisons.

1. Comparison 1: special manual toothbrushes versus conventional manual toothbrushes for people with ID.

2. Comparison 2: electric toothbrushes versus manual toothbrushes for people with ID.

3. Comparison 3: oral hygiene care training versus no oral hygiene care training or a placebo training session for carers of people with ID.

4. Comparison 4: oral hygiene training versus no oral hygiene training of people with ID.

One study evaluated each of the following comparisons.

1. Comparison 5: one-, three- and six-monthly dental recall intervals plus daily supervised brushing versus usual care for people with ID.

2. Comparison 6: discussion of individual clinical photographs as a toothbrushing motivator versus no discussion of photographs for people with ID.

3. Comparison 7: daily, twice-weekly and once-weekly frequencies of teeth brushing versus usual care for people with ID.

4. Comparison 8: use of a toothpaste with a plaque-disclosing agent versus a conventional toothpaste for people with ID.

5. Comparison 9: individualised oral care plan versus usual care (according to guidelines) for people with ID.

\section{Interventions involving electric and manual toothbrushes}

Thirteen studies compared an electric toothbrush to a manual toothbrush, eight RCTs and five NRS (Bratel 1991; Gertenrich 1967a; Gertenrich 1967b; Gertenrich 1967c; Gertenrich 1967d). Four RCTs were cross-over trials: one had two arms (Shaw 1983), and the others had three arms; the third arm being a special manual toothbrush (Christen 2007; Dôgan 2004; Kaschke 2005). The other four RCTs were Carr 1997; Garcia-Carrillo 2016; Kelner 1963; and Swallow 1969.

Seven studies compared a special manual toothbrush with a conventional toothbrush. There were six RCTs: three, three-armed cross-over trials (Christen 2007; Dôgan 2004; Kaschke 2005), and three others (Bildt 2010; Ferozali 2007; Sauvetre 1995). One NRS compared a special manual toothbrush with a conventional toothbrush (Williams 1988).

The studies comparing electric toothbrushes to manual toothbrushes used a variety of electric toothbrushes: Oral B Braun (3D) (Bratel 1991; Dôgan 2004), Teledyne Waterpik Sonic Speed (Kaschke 2005), Panasonic Dentacare-Sonodent (Christen 2007), Interplak (Carr 1997), Phillips Sonicare EasyClean (Garcia-Carrillo 2016), Oral B Arcuate Action (Gertenrich 1967a; Gertenrich 1967b; Gertenrich 1967c), and Broxodent (Kelner 1963). Shaw 1983 did not identify the electric toothbrush used.

The studies comparing special manual toothbrushes also used a variety of toothbrushes: Collis Curve (Williams 1988), Superbrush (Bildt 2010; Christen 2007; Dôgan 2004; Kaschke 2005; Sauvetre 1995), and a single-use suction toothbrush (Ferozali 2007).
Participants with ID brushed their own teeth in five of these studies (Dôgan 2004; Garcia-Carrillo 2016; Gertenrich 1967d; Sauvetre 1995; Shaw 1983) and carers (or others) brushed the teeth of those with ID in seven studies (Bildt 2010; Ferozali 2007; Gertenrich 1967b; Gertenrich 1967c; Kelner 1963; Swallow 1969; Williams 1988). Five studies were mixed: they distinguished between "self-brushers," "assisted brushers" and "carer brushed" (Bratel 1991; Carr 1997; Christen 2007; Gertenrich 1967a; Kaschke 2005); we combined these groups for meta-analysis.

Interventions involving training of carers and people with intellectual disabilities

Four RCTs (Gonzalez 2013; Mac Giolla Phadraig 2015; Olmos 2016; Phlypo 2016) and three NRSs (Glassman 2006; Kissel 1983; Lange 2000) focused on the training of carers to improve the oral health of the people with ID for whom they cared. The descriptions of the training provided lacked detail; those that indicated the duration of the training delivered it in one session, which varied in length: 90 minutes (Gonzalez 2013), three hours (Glassman 2006), or one day (Mac Giolla Phadraig 2015). Some interventions provided training manuals for the carers (Mac Giolla Phadraig 2015; Phlypo 2016), while others provided continual feedback based on observation (Glassman 2006; Lange 2000), video recordings (Kissel 1983), or monitoring of daily plaque scores (Lange 2000). Some interventions included practical skills training as well as theory, which was either delivered as role play (Mac Giolla Phadraig 2015), demonstrated on one of the participants (Lange 2000), or individualised for each carer and person with ID (Kissel 1983). The timing of the evaluation of the intervention varied: immediately after the delivery of the training (Gonzalez 2013), four weeks (Olmos 2016), eight weeks (Phlypo 2016), 100 days (Kissel 1983), 156 days (Glassman 2006), and 11 months (Mac Giolla Phadraig 2015). The training was delivered by dental professionals except in Kissel 1983, where it was delivered by a psychologist.

Two RCTs (Albino 1979; Lange 1985) and four NRS (Abramson 1972; Bouter 1979; Jarman 1983; Snell 1989) focused on the training of people with ID to improve their oral health. The interventions focused on training people with ID in the practical skills of toothbrushing, with only one providing some theory in relation to the concepts of oral health (Albino 1979). The participants' levels of ID spanned the entire range: mild/moderate (Lange 1985), moderate (Bouter 1979), moderate/severe (Abramson 1972; Albino 1979), severe (Jarman 1983), and severe/profound (Snell 1989). Snell 1989 involved the participants in partial participation: the carers performed the toothbrushing and the participants were trained to assist where possible in the routine. In Jarman 1983, training in five other self-care routines was included in the intervention.

The duration of the training for people with ID tended to be considerably longer than that for carers; the shortest period was two weeks (Lange 1985) and the longest 19 months (Snell 1989). However, this varied from participant to participant depending on their particular needs and some interventions included a maintenance phase, where the trainer prompted the participant to undertake the next step in the routine.

The description of the toothbrushing routine varied from "task completion" (Albino 1979; Jarman 1983), to broad stages (Lange 1985), to detailed step-by-step descriptions (Abramson 1972; Bouter 1979; Snell 1989). One study used disclosing tablets to 
indicate plaque as a teaching and monitoring aid (Lange 1985). All the interventions used praise as a motivator; three studies also included physical rewards or tokens (Abramson 1972; Albino 1979; Jarman 1983). Four studies led or delivered training by a psychologist (Abramson 1972; Albino 1979; Bouter 1979; Jarman 1983), one study by a dentist (Lange 1985), and one study by a teacher (Snell 1989).

\section{Interventions assessed in one study only}

One RCT evaluated dental recall intervals (one-monthly, threemonthly, six-monthly) and daily supervised toothbrushing versus usual care (Shaw 1991), one RCT evaluated using an individual's clinical photographs to discuss oral hygiene performance versus not discussing them (Bickley 1990), and one RCT compared brushing daily by a dental professional to brushing twice weekly or once weekly (Swallow 1969). One NRS compared using a toothpaste with a plaque-disclosing agent versus a conventional toothpaste (Teitelbaum 2009), and another NRS compared the use of an individualised oral care plan versus usual care (Altabet 2003).

\section{Characteristics of outcomes}

The clinical outcomes assessed for the participants in the RCTs were: plaque, oral hygiene status (subjective), gingival inflammation, gingival bleeding, calculus, oral assessment, total bacterial count and potential pathogenic bacteria count. The behavioural outcomes assessed for the participants or the carers (or both) were knowledge, behaviour, attitude and self-efficacy.

The outcomes assessed for the participants in the NRS were: plaque, oral hygiene status, gingival inflammation, gingival bleeding, gingival pocketing, calculus, self-efficacy (steps in a toothbrushing routine), duration of the toothbrushing episode, individually tailored expected toothbrushing performance and level of "self-initiation." The outcomes assessed for the carers in the NRS were: need for verbal instruction and physical guidance, carer presence during toothbrushing by a person with ID and carer use of verbal instruction, physical guidance and rewards.

\section{Timing of outcome measurement}

Thirteen studies reported outcomes at more than one time point, which were, for the most part, within the same time frame: short term (Lange 1985; Lange 2000; Sauvetre 1995) or medium term (Carr 1997; Ferozali 2007; Garcia-Carrillo 2016; Gertenrich 1967a; Gertenrich 1967b; Gertenrich 1967c; Gertenrich 1967d; Jarman 1983). We selected the longest time points for each study that were common among the studies for each of the time periods (short, medium or long term). Two studies reported findings across the time frames. Bratel 1991 reported findings in the short (one and four weeks), medium (12 weeks) and long term (16 months); we used the data from the four-week, 12 -week and 16-month time points for analysis. Shaw 1991 reported findings in the medium and long term (three, six, 12, 18 and 24 months); we used only data from the 24month time point for analysis as the participant numbers were not provided for any other time points.

\section{Gingival health}

\section{Gingival inflammation}

Twelve RCTs reported gingival inflammation, 11 of which used a validated gingival index. The indices used were Silness \& Löe (Carr 1997; Garcia-Carrillo 2016; Lange 1985; Phlypo 2016; Swallow 1969), Lobene (Mac Giolla Phadraig 2015), WHO Technical Report 621 Gingival Index (Shaw 1983), Saxer \& Muhlemann Papillary Bleeding Index (Christen 2007; Kaschke 2005; Sauvetre 1995), and Ainamo \& Bay (Bildt 2010). The other RCT used the subjective rating of "worse, same or better" (Kelner 1963).

Six NRS reported gingival inflammation; two studies used a validated gingival index: Silness \& Löe (Bratel 1991) and the WHO Technical Report 621 Gingival Index (Shaw 1991). The four studies using a subjective rating of gingival inflammation used the terms "obvious gingivitis, decrease in gingivitis or absence of clinically observable gingivitis" (Gertenrich 1967a; Gertenrich 1967b; Gertenrich 1967c; Gertenrich 1967d).

Modifications to the gingival indices were made in several studies; the most common modification was in relation to the number of teeth and surfaces examined. Thirteen studies used six standard teeth on the following surfaces.

\section{Surfaces assessed on 6 standard teeth for gingival inflammation}

4 buccal and 2 lingual surfaces

\section{Studies}

Bildt 2010; Carr 1997; Mac Giol-

la Phadraig 2015; Phlypo 2016;

Teitelbaum 2009

The Bentley 1995 modification; 2 randomly selected (by coin toss) quadrants (1 in the upper jaw, 1 Garcia-Carrillo 2016 in the lower jaw, contralateral)

\begin{tabular}{ll}
\hline 4 surfaces per tooth & Bratel 1991 \\
\hline 3 surfaces per tooth; buccal, mesial and lingual & Sauvetre 1995 \\
\hline 2 surfaces per tooth; on the buccal and lingual surfaces & Christen 2007; Kaschke 2005 \\
\hline 2 surfaces per tooth; on the mesial and distal surfaces of each tooth & Lange 1985; Lange 2000 \\
\hline The interdental papillae and the intervening gingiva on the buccal surfaces of all standing teeth & Swallow 1969 \\
\hline
\end{tabular}

Oral hygiene interventions for people with intellectual disabilities (Review) 


\section{Other modifications}

No probe used and only teeth that were visible to the examiner

\section{Studies}

Mac Giolla Phadraig 2015

If standard teeth were missing they were not substituted

Lange 2000

Only two studies (Shaw 1983; Shaw 1991) reported carrying out a periodontal pocket index assessment (WHO 1978) and only one of these, an NRS, reported the findings (Shaw 1991).

\section{Plaque}

Fourteen RCTs reported plaque as an outcome using a validated index. The indices used were Silness \& Löe (Garcia-Carrillo 2016; Phlypo 2016; Sauvetre 1995; Shaw 1983; Swallow 1969), Green \& Vermillion (Bildt 2010; Carr 1997; Lange 1985), Quigley-Hein (Christen 2007; Dôgan 2004; Kaschke 2005), Kobayashi \& Ash (Albino 1979), the WHO Technical Report No 621 (Bickley 1990), Approximal Plaque Index (Christen 2007; Dôgan 2004; Kaschke 2005), and Shaw \& Murray (Bickley 1990). One RCT used a subjective rating of oral hygiene describing the outcome using the terms "worse," "same" or "better" (Kelner 1963).
Six NRS reported plaque as an outcome using a validated index; the indices used were Silness \& Löe (Bratel 1991), Green \& Vermillion (Glassman 2006; Teitelbaum 2009; Williams 1988), Ramfjord (Lange 2000) and the WHO Technical Report No 621 (Shaw 1991). A further five NRS used a subjective rating describing the outcome using the following terms: "excellent," "good," "fair," "poor" or "very poor" (Altabet 2003); "good," "fair," "poor" or "very poor" (Gertenrich 1967a; Gertenrich 1967b; Gertenrich 1967c; Gertenrich 1967d).

Four studies used more than one measure of plaque. Modifications to the indices were made in several studies, most commonly in relation to the number of teeth and surfaces examined. Sixteen studies used six standard teeth on the following surfaces.

\section{Surfaces assessed on 6 standard teeth for plaque levels}

\section{Studies}

4 buccal and 2 lingual surfaces

Bickley 1990; Carr 1997; Glassman 2006;

Mac Giolla Phadraig 2015; Phlypo 2016; Shaw 1983; Shaw 1991; Teitelbaum 2009; Williams 1988

\section{The Bentley 1995 modification; 2 randomly selected (by coin toss) quadrants ( 1 in the Garcia-Carrillo 2016} upper jaw, 1 in the lower jaw, contralateral)

\begin{tabular}{ll}
\hline 4 surfaces per tooth & Bratel 1991 \\
\hline 3 surfaces per tooth; buccal, mesial and lingual & Sauvetre 1995 \\
\hline 2 surfaces per tooth; on the buccal and lingual surfaces & Albino 1979; Bildt 2010 \\
\hline 2 surfaces per tooth; on the mesial and distal surfaces of each tooth & Lange 1985; Lange 2000 \\
\hline Other modifications & Studies \\
\hline No probe used and only teeth that were visible to the examiner & Mac Giolla Phadraig 2015 \\
\hline If standard teeth were missing they were not substituted & Lange 2000 \\
\hline
\end{tabular}

\section{Calculus}

Four studies reported on calculus; three RCTs and one NRS. The indices used were: Green \& Vermillion (Carr 1997; Garcia-Carrillo 2016) and the WHO Technical Report 621 (Bickley 1990 (assessed but not reported); Shaw 1991). All three studies that reported their findings used the six standard teeth. Two studies assessed four buccal and two lingual surfaces and one study assessed four surfaces per tooth (buccal, lingual, mesial and distal).

\section{Oral assessment}

Ferozali 2007 used a tool to assess the health of four elements; lips, oral mucosa, gingivae and plaque, which was a modification of the Beck Total Oral Assessment Tool. This study also measured a total bacteria count and a potentially pathogenic bacteria count.

The following are the references for the clinical indices referred to in this section: Ainamo 1975; Beck 1993; Bentley 1995; Fleiss 1987; 
Greene 1960; Kobayashi 1964; Lange 1977; Lobene 1986; Löe 1967; Saxer 1975; Shaw 1977; WHO 1978.

\section{Oral hygiene knowledge acquisition}

Three RCTs assessed changes in oral healthcare knowledge of carers, all using multiple-choice questionnaires, two of which were specifically designed by the investigators for the intervention (Gonzalez 2013; Mac Giolla Phadraig 2015), and the third (Phlypo 2016) used the questionnaire designed by Mac Giolla Phadraig 2015. Two studies had 10 questions on oral health-related knowledge (Mac Giolla Phadraig 2015; Phlypo 2016), and one study had 20 basic oral hygiene questions (Gonzalez 2013).

The Mac Giolla Phadraig 2015 questionnaire was reported as having been tested for reliability, validity, responsiveness and reproducibility and found to be acceptable. Gonzalez 2013 used a different questionnaire pre- and postintervention, with the same content but different wording; the authors sought expert advice to assess the content.

\section{Changes in behaviour, attitude and self-efficacy}

Two of the RCTs that assessed knowledge of carers also assessed changes in their behaviour, attitude, self-efficacy or a combination of these at the same time: the studies had four questions on behaviour and five each on attitude and self-efficacy (Mac Giolla Phadraig 2015; Phlypo 2016). One RCT assessed changes in selfreported behaviour alone, using five questions (Olmos 2016). Four other NRS assessed behavioural changes in both carers and people with ID by monitoring of their behaviour using trained observers aided by specifically designed checklists (Bouter 1979; Glassman 2006; Jarman 1983; Kissel 1983). Reliability of the recorded observations was assessed by the study authors and was found to be acceptable in all four of these studies.

The methods used to assess changes in behaviour included: use of different methods of reinforcement by carers (Jarman 1983); assessing staff use of training methods when providing oral hygiene support (verbal instruction, physical guidance and reward) and participants' levels of self-initiation and their need for verbal instruction and physical guidance (Kissel 1983); the effect of training on carers and levels of feedback and support to them (Abramson 1972); behavioural methods used by carers to assist people with ID (Bouter 1979); the type of toothbrush used, the type of toothpaste used, the frequency of fluoride application, the timing of toothbrushing and duration of toothbrushing (Olmos 2016); carers' presence during toothbrushing (Glassman 2006); and the number of training sessions required for the toothbrushing skill to be performed without prompting (Snell 1989).

Indirect measures of behaviour change included measuring wear and tear on toothbrushes and a diary kept by the parents/staff of the participants' brushing frequency (Bratel 1991). Bildt 2010 assessed ease of toothbrush use by carers and toothbrushing frequency, as well as degree of resistance to toothbrushing by people with ID.

\section{Quality of life changes or long-term, patient-centred outcomes}

No studies measured quality of life changes or long-term, patientcentred outcomes as formal outcomes.

\section{Dental caries levels}

No studies measured dental caries levels.

\section{Any unintended (positive or negative) effects of the interventions}

\section{Positive effects}

No studies reported any positive unintended effects.

\section{Negative (adverse) effects}

Twenty-four studies did not report any unintended negative effects (or an intention to measure them from the beginning of the study). Ten studies investigated or reported negative unintended or adverse effects.

\section{Subjective outcomes}

Several subjective outcomes were reported narratively, in relation to possible benefits or harm, for example, interviews with people using an electric toothbrush to assess their experiences using it (Bratel 1991); feedback from carers on the toothbrushes used (Gertenrich 1967a; Gertenrich 1967b; Gertenrich 1967c; Gertenrich 1967d); carer questionnaire about experience of using a special three-headed toothbrush (Bildt 2010); social validation of participants' abilities with regard to oral hygiene completed by their carers and carers' perception of training (Glassman 2006); a staff acceptability questionnaire (Kissel 1983); and questions on participants' skill retention (Snell 1989).

\section{COM-B system characteristics}

Most studies did not define behavioural change theories in the interventions. Therefore, we assessed all of the studies using the COM-B system to identify the BCTs inherent in the interventions (Michie 2011). We considered how the interventions affected Capability (physical strength, skill or stamina or psychological strength, knowledge, skill or stamina), Opportunity (physical opportunity afforded by the environment or the social opportunity afforded by social factors such as cultural norms or social cues) and Motivation (reflective brain process; evaluation and plans or automatic brain processes; desires, impulses, inhibitions, etc.).

The element we identified most often was capability: 29 studies had the potential to change the capability of either participants (11 studies) or carers (eight studies) or both (10 studies), either on a physical level (11 studies) or on a physical and psychological level (18 studies). These interventions included a focus on training, modelling or enablement of the participant or carer in the skill of toothbrushing.

In relation to the element of opportunity, physical opportunity was the more common potential element identified ( 26 studies). These interventions included elements such as specific time being set aside for the toothbrushing routine, environmental restructuring such as providing different oral hygiene or other aids or changing the setting where the toothbrushing took place. The potential for social opportunity (e.g. cultural norms or social cues) to impact on behaviour was identified in seven studies, which were predominantly targeted at carers and involved monitoring of their behaviour.

Nineteen studies had the potential for reflective motivation and these tended to include some element of feedback in relation to behaviour or performance which was seen predominantly in the interventions targeting carers. No studies had the potential for the behaviour change to become automatic. 
We determined that 16/34 studies had all three elements of capability, opportunity and motivation inherent in the intervention.

The behavioural change elements and intervention functions are detailed in the Characteristics of included studies table.

\section{Stakeholder involvement}

The involvement of stakeholders may impact on the success of an intervention and so it was felt important to this review to capture the nature of the stakeholder involvement in the interventions. Twenty-nine studies actively involved parents or carers of the participants in the intervention. Six studies involved both formal and non-formal carers, 22 studies involved formal carers only and one study used non-formal carers only. Three other studies were unclear if carers were involved. These studies were set in a school or day centre (Bickley 1990; Dôgan 2004; Sauvetre 1995). Two studies used a member of the research team to brush the participants' teeth (Swallow 1969; Williams 1988). Other stakeholders included administration staff, management staff, nursing or medical staff, speech and language therapists, an occupational therapist, a physical therapist and teachers. Eight studies reported no involvement of stakeholders, other than those directly involved in the research.

Twenty-six studies had dental professionals on the research team, 21 studies involved dentists or dental students, 16 studies involved dental hygienists or therapists, four studies involved dental nurses or assistants and one study involved an oral health promotor. The dental professionals were mainly involved in the initial oral hygiene instruction and the clinical measurements throughout the intervention. Seven studies indicated that the dental professional provided ongoing support during the intervention (Albino 1979; Bickley 1990; Glassman 2006; Sauvetre 1995; Shaw 1991; Swallow 1969; Williams 1988); one other study indicated that this support was offered but not if the offer was taken up (Mac Giolla Phadraig 2015).

\section{Funding}

Nine studies reported receiving funding from their institution or health service (Abramson 1972; Bratel 1991; Gonzalez 2013; Jarman 1983; Olmos 2016; Kissel 1983; Mac Giolla Phadraig 2015; Shaw 1991; Teitelbaum 2009). Sixteen studies did not report on funding. Eight studies reporting receiving some funding from dental companies; all funding was stated as relating to the supply of either the toothbrushes or toothpaste required for the study (Albino 1979; Bickley 1990; Dôgan 2004; Garcia-Carrillo 2016; Kelner 1963; Sauvetre 1995; Shaw 1991; Swallow 1969).

\section{Excluded studies}

Following full-text reading, we rejected 158 reports for the following reasons: not an eligible study design (75 studies), not an oral hygiene intervention (39 studies), not mechanical removal of plaque (16 studies), a complex intervention in which the oral hygiene component could not be appraised separately (11 studies) and not relating to a person with ID or their carer (17 studies).

We excluded a further 20 reports after further consideration for the following reasons. We excluded two as only the title was available (Borglin 1969; Lopez 1994) and further five as only the abstract was available (Andrews 1990; Badra 1973; Ojeda 2010;
Ribeiro 2011; Thornton 1991), and multiple efforts to contact the authors and source the full text were unsuccessful. A further three reports presented no data in the published reports or data were presented in a format that could not be used and attempts to contact the authors were unsuccessful (Brody 1975; Favell 1975; Meador 1979). The authors of three other reports with data in a format that could not be used were contacted but the data were no longer available (Gertenrich 1972; Lesmana 2014; Schmidt 1981). Two other reports were trial registers, one had been written up in a thesis but was unavailable and all attempts to contact the author were unsuccessful (ISRCTN10044161), and the authors of the second trial reported that the trial had not proceeded as yet, due to difficulties in recruiting participants (NCT03234231). One report was excluded as it was unclear if it met the inclusion criteria in relation to study design; all attempts to contact the authors were unsuccessful (Horner 1975). Three studies did not meet the required study design (Haran 2014; Kaschke 2008; Zaksek 2014). One of the authors of another study, with which might have had a subgroup of participants who may have been eligible for inclusion, confirmed that the study did not meet the study design criteria (Bui 2003) (see Characteristics of excluded studies table).

Two studies are awaiting classification (Akgun 2012; Birani 2008). We made contact with the authors as the theses for both are available, but, despite reminders, the information was not received before the deadline indicated for inclusion in this review.

\section{Risk of bias in included studies}

We assessed the RCTs and NRCTs for risk of bias using standardised risk of bias criteria (Higgins 2011a). We assessed the ITS and RM studies using the EPOC seven standard criteria (EPOC 2016) (Figure 1; Figure 2).

Of the 28 RCTs and NRCTs assessed, none were at overall low risk of bias. Eighteen were at overall high risk of bias (Albino 1979; Altabet 2003; Bickley 1990; Bratel 1991; Christen 2007; Dôgan 2004; Gertenrich 1967a; Gertenrich 1967b; Gertenrich 1967c; Gertenrich 1967d; Kaschke 2005; Kelner 1963; Lange 1985; Lange 2000; Mac Giolla Phadraig 2015; Phlypo 2016; Shaw 1983; Williams 1988); the remaining 10 were at unclear risk of bias overall.

All of the ITS and RM studies were at high risk of bias overall (Abramson 1972; Bouter 1979; Glassman 2006; Jarman 1983; Kissel 1983; Snell 1989).

\section{Allocation}

Of the 19 RCTs included in this review, 11 were at low risk of bias in relation to random sequence generation (Albino 1979; Bickley 1990; Bildt 2010; Christen 2007; Ferozali 2007; GarciaCarrillo 2016; Gonzalez 2013; Mac Giolla Phadraig 2015; Sauvetre 1995; Shaw 1983; Swallow 1969). These studies clearly indicated the method used for randomisation. A further 10 RCTs stated that the participants were randomised but gave no details of how this randomisation process was carried out. These studies were at unclear risk of bias. No RCTs were at high risk of bias. The allocation concealment rating for the RCTs was nine studies at low risk of bias and 10 studies were at unclear risk of bias.

The nine NRCTs were assessed using the RCT risk of bias criteria (Higgins 2011a). Six of these studies were at high risk of bias for random sequence generation as the participants were selected non-randomly or the participants were selected for particular 
groups; the remaining three studies were at unclear risk of bias as there was no reference to randomisation. One NRCT was at low risk of bias (Lange 2000); two NRCTs were at high risk of bias for allocation concealment as participants were either assigned to groups based on the date they attended or based on their need for assistance (Altabet 2003; Bratel 1991). The remaining six NRCTs were at unclear risk of bias for allocation concealment as there were no details in the reports in relation to this element.

\section{Blinding}

Four RCTs were at high risk of bias in relation to blinding of participants or personnel (or both): all four were cross-over trials (Christen 2007; Dôgan 2004; Kaschke 2005; Shaw 1983). We considered five RCTs to be at low risk as the control groups were asked to do something that was not usual care (Bildt 2010; Gonzalez 2013; Kelner 1963; Sauvetre 1995; Shaw 1991). The remaining 10 RCTs were at unclear risk of bias.

One NRCT was at high risk of bias in relation to blinding of participants or personnel (or both) (Williams 1988). Three NRCTs were at low risk of bias as some attempt was made to blind either the participants or personnel (Altabet 2003; Lange 2000; Teitelbaum 2009). The remaining five NRCTs were at unclear risk of bias for blinding of participants or personnel (or both) (Bratel 1991; Gertenrich 1967a; Gertenrich 1967b; Gertenrich 1967c; Gertenrich 1967d).

In relation to blinding of outcome assessors in the RCTs, 10 studies were at low risk of bias as the assessors were blinded to the group allocation (Bickley 1990; Bildt 2010; Christen 2007; Ferozali 2007; Garcia-Carrillo 2016; Kaschke 2005; Kelner 1963; Sauvetre 1995; Shaw 1983; Swallow 1969); seven studies were at unclear risk of bias as there were insufficient details provided in the reports in relation to blinding (Carr 1997; Dôgan 2004; Gonzalez 2013; Mac Giolla Phadraig 2015; Olmos 2016; Phlypo 2016; Shaw 1991); two studies were at high risk of bias as the assessors were not blinded to the group allocation (Albino 1979; Lange 1985).

In the NRCTs, six studies were at low risk of bias as assessors were blinded to the group allocation; the remaining three studies were at unclear risk of bias as no mention of blinding of assessors was reported (Bratel 1991; Teitelbaum 2009; Williams 1988).

\section{Incomplete outcome data}

Four RCTs were at high risk of bias in relation to incomplete outcome data as either there were high attrition rates with no explanation as to the reasons for the dropouts (Mac Giolla Phadraig 2015; Phlypo 2016; Shaw 1983) or dropouts were due to lack of participation by the participants (Albino 1979). Three studies were at unclear risk of bias as either there was insufficient detail in relation to the reason or number of dropouts (Dôgan 2004), or the reasons provided may have impacted on the outcomes: participants lost or broke the electric toothbrush (Carr 1997), or the participants were unable to following the training (Sauvetre 1995). The remaining 12 studies were at low risk of bias for incomplete outcome data.

One NRCT was at unclear risk in relation to incomplete outcome data due to the lack of details provided (Gertenrich 1967b). The remaining eight NRCTs were at low risk of bias.

\section{Selective reporting}

Three RCTs were at high risk of bias in relation to selective reporting: one because the plaque index was modified during the intervention as it proved too difficult to complete (Albino 1979); two because all the outcomes outlined in the methodology were not reported (Bickley 1990; Kelner 1963). Fourteen studies were at unclear risk of bias as there was no protocol for the study but all listed outcomes were reported. Another study referred to mid-term data but they were not reported (Shaw 1983), and one study had a protocol but it appeared to have been completed retrospectively (Garcia-Carrillo 2016). No studies were at low risk of bias in relation to selective reporting.

All nine NRCTs were at unclear risk of bias for selective reporting as there was no protocol but all the expected outcomes reported in the methodology sections were reported as planned.

\section{Other potential sources of bias}

There were no other potential sources of bias.

Overall, there was a large element of 'unclear' risk of bias in many of the domains, reflecting the lack of detail provided in the published reports in relation to the methodology. This may be due to the large number of older studies included in this review. There were no studies at low risk of bias for the domain of selective reporting: many of the studies had no protocol and so were assessed at unclear risk of bias for this domain.

\section{Risk of bias for interrupted time series and repeat measure studies}

Three ITS studies (Glassman 2006; Jarman 1983; Kissel 1983) and three RM studies (Abramson 1972; Bouter 1979; Snell 1989) were assessed for risk of bias using the Effective Practice and Organisation of Care (EPOC) Group standards (EPOC 2016).

\section{Was the intervention independent of other changes?}

Of the six studies, two were independent of other changes (low risk) (Bouter 1979; Glassman 2006); three were unclear in relation to being independent of other changes as there was insufficient information (Abramson 1972; Kissel 1983; Snell 1989); and one was not independent (high risk), as the skill of toothbrushing was one of six other skills being taught at the same time (Jarman 1983).

\section{Was the shape of the intervention effect prespecified?}

All six ITS and RM studies had prespecified the shape of the intervention in relation to the methodology and expected outcomes (low risk).

\section{Was the intervention unlikely to affect data collection?}

The intervention in all six studies was unlikely to affect data collection (low risk).

\section{Was knowledge of the allocated interventions adequately prevented during the study?}

It was not possible to prevent knowledge of the allocated intervention in the six studies as the assessors in all cases were actively involved in the delivery of the intervention (high risk). 


\section{Were incomplete outcome data adequately addressed?}

Incomplete data were explained in two studies and had no impact on the outcomes (Bouter 1979; Kissel 1983); there were no incomplete data in the other four studies (low risk).

\section{Was the study free from selective outcome reporting?}

Five studies were free from selective outcome reporting as all outcomes outlined in the methodology were reported (low risk). One study was unclear as it was difficult to extract the data from the figures provided (Glassman 2006).

\section{Was the study free from other risks of bias?}

No other risks of bias were identified.

\section{Effects of interventions}

See: Summary of findings for the main comparison Special manual toothbrush compared to conventional manual toothbrush for people with intellectual disabilities; Summary of findings 2 Electric toothbrush compared to manual toothbrush for people with intellectual disabilities; Summary of findings 3 Training of carers compared to no training of carers for people with intellectual disabilities; Summary of findings $\mathbf{4}$ Oral hygiene training compared to no oral hygiene training for people with intellectual disabilities; Summary of findings $\mathbf{5}$ Regularly scheduled dental visits and daily supervised toothbrushing compared to usual care for people with intellectual disabilities; Summary of findings 6 Discussion of clinical photographs as oral hygiene motivators compared to no discussion for people with intellectual disabilities; Summary of findings 7 Daily toothbrushing compared to twiceweekly or once-weekly toothbrushing for people with intellectual disabilities; Summary of findings $\mathbf{8}$ Toothpaste with a plaquedisclosing agent compared to a conventional toothpaste for children with intellectual disabilities; Summary of findings 9 Individualised care plans compared to usual care for people with intellectual disabilities

\section{Comparison 1: special manual toothbrushes versus conventional manual toothbrushes for people with intellectual disabilities}

Six RCTs compared a special manual toothbrush with a conventional toothbrush (Bildt 2010; Christen 2007; Dôgan 2004; Ferozali 2007; Kaschke 2005; Sauvetre 1995). Five studies used the three-headed Superbrush, while Ferozali 2007 used a single-use suction toothbrush. One NRS evaluated a toothbrush with curved bristles (the Collis Curve) (Williams 1988). See Summary of findings for the main comparison.

\subsection{Gingival inflammation (short term)}

Three RCTs, with 169 participants, compared using a special manual toothbrush with using a conventional manual toothbrush on gingival inflammation over two and three weeks (Christen 2007; Kaschke 2005; Sauvetre 1995; Table 1). Two studies were three-armed cross-over trials; the third arm compared an electric toothbrush (reported separately). The special brush used in all three studies was the Superbrush. The parallel-group study showed no evidence of a difference between the two toothbrushes for people with ID in the short term (MD $-0.10,95 \% \mathrm{Cl}-0.77$ to 0.57 ; Analysis 1.1; Sauvetre 1995). The two cross-over RCTs also reported no difference between the toothbrushes. The level of participant ID was mixed or unclear. The certainty of the evidence was low.

\subsection{Gingival inflammation (medium term)}

One RCT, with 18 participants, compared the effect of carers using a special manual toothbrush (Superbrush) to brush the teeth of people with moderate-to-profound ID compared with a conventional manual toothbrush on gingival inflammation levels over four months (Bildt 2010; Table 2). It showed a large effect size in favour of the special manual toothbrush for adults with ID in the medium term (four months) when the toothbrushing was carried out by carers (MD $-12.40,95 \% \mathrm{Cl}-24.31$ to -0.49 ; Analysis 1.2 ). The certainty of the evidence was very low.

\subsection{Plaque (short term)}

Four RCTs, with 189 participants aged between four and 79 years, compared using a special manual toothbrush with using a conventional manual toothbrush on plaque levels over periods of one, two and three weeks (Christen 2007; Dôgan 2004; Kaschke 2005; Sauvetre 1995). The special brush used in all four studies was the Superbrush. Three studies were three-armed cross-over trials; the third arm compared an electric toothbrush (reported separately) (Table 3).

One parallel-group RCT measured plaque at 21 days and found no evidence that either toothbrush was better in the short term (MD $0.20,95 \% \mathrm{Cl}-0.45$ to $0.85 ; 25$ participants; Analysis 1.3; Sauvetre 1995). The level of ID of the participants was unclear in this study. One cross-over RCT, with participants who had mixed levels of ID, reached the same conclusion when measuring plaque at two weeks (Christen 2007). Some evidence favouring the special manual toothbrush was found in two RCTs: in Dôgan 2004, which measured plaque at one week in participants with mild ID, and in Kaschke 2005, a crossover study of participants with mixed levels of ID that measured plaque at two weeks. The certainty of the evidence was very low.

\subsection{Plaque (medium term)}

One RCT, with 18 participants with moderate to severe ID, compared the effect of formal carers using a special manual toothbrush (Superbrush) to brush the teeth of people with ID compared with a conventional manual toothbrush on plaque levels, over four months (Bildt 2010; Table 4). The baseline scores were not equal and the assessors were not calibrated introducing an element of indirectness in relation to certainty of the evidence. This study suggested there may be a greater reduction in plaque with the special manual toothbrush (Superbrush) for people with ID in the medium term, when the toothbrushing was carried out by formal carers; however, the result was also compatible with no difference between the interventions (MD $-0.44,95 \% \mathrm{Cl}-0.93$ to 0.05 ; Analysis 1.4). The certainty of the evidence was very low.

One NRS found no evidence of the superiority of either a special (Collis Curve toothbrush) or a conventional manual toothbrush (Williams 1988; Table 5). Participants in this study had profound ID and their teeth were brushed by a student dental assistant.

\subsection{Oral health assessment and bacteria levels (medium term)}

One RCT, with 22 participants, compared using a special manual toothbrush (single-use suction toothbrush with a therapeutic chemical agent) with using a conventional manual toothbrush (and the same therapeutic chemical agent) on general oral health and oral bacteria levels over 90 days (Ferozali 2007). All the participants had a profound level of ID and had their teeth brushed by a carer. 
None of the assessments provided evidence of one toothbrush being superior to the other (Analysis 1.5; Table 6). The certainty of the evidence was very low.

\subsection{Changes in behaviour, attitude and self-efficacy}

One RCT compared the effect of carers using a special manual toothbrush (Superbrush) to brush the teeth of people with moderate-to-profound ID compared with using a conventional manual toothbrush over four months (Bildt 2010; Table 7). The certainty of the evidence was very low. The analyses of the carer elements in this single trial found no significant difference between toothbrushes for the carer experience when handling the toothbrush and when brushing the teeth of people with ID in the medium term (handling the toothbrush: MD $0.50,95 \% \mathrm{Cl}-0.35$ to 1.35 ; brushing the teeth: MD $0.70,95 \% \mathrm{Cl}-0.15$ to 1.55$)$. There was no evidence of a difference between the toothbrushes in terms of frequency of brushing by the carer (MD $0.20,95 \% \mathrm{Cl}-2.13$ to 2.53; Analysis 1.6). A meta-analysis of the resistance of people with ID to toothbrushing found no evidence of a significant difference between the toothbrushes (MD $-1.70,95 \% \mathrm{Cl}-4.90$ to 1.50; Analysis 1.7).

\subsection{Adverse effects}

Bildt 2010, and Christen 2007 reported that the size of the threeheaded toothbrush caused problems when first used or for people with limited mouth opening. Ferozali 2007 considered the allergy potential of the chemical agents being used on the toothbrushes at the outset of the study and reported there were no allergic reactions. The other studies did not report any adverse effects.

\section{Comparison 2: electric toothbrushes versus manual toothbrushes for people with intellectual disabilities}

Eight RCTs (Carr 1997; Christen 2007; Dôgan 2004; Garcia-Carrillo 2016; Kaschke 2005; Kelner 1963; Shaw 1983; Swallow 1969), and five NRSs (Bratel 1991; Gertenrich 1967a; Gertenrich 1967b; Gertenrich 1967c; Gertenrich 1967d) compared electric with manual toothbrushes. See Summary of findings 2 .

\subsection{Gingival inflammation (short term)}

Four RCTs, with 270 participants, compared using an electric toothbrush with using a manual toothbrush on gingival inflammation, over two, three or four weeks (Christen 2007; Kaschke 2005; Shaw 1983; Swallow 1969). The level of participant ID was mixed. Three studies were cross-over trials, two included a third arm, which also compared a special manual toothbrush (reported earlier).

There was no strong evidence of a difference between the electric and manual toothbrushes for levels of gingival inflammation short term in any of the studies (Table 8). None of the studies presented data in a format that could be included in a meta-analysis. The certainty of the evidence was low.

\subsection{Gingival inflammation (medium term)}

Three RCTs, two of which were cluster RCTs, with 220 participants, compared using an electric toothbrush with using a manual toothbrush on gingival inflammation levels over periods of three, four, six and 12 months (Carr 1997; Garcia-Carrillo 2016; Kelner 1963; Table 9).
We combined the cluster RCTs in a meta-analysis, which showed no evidence of a difference in gingival inflammation levels between toothbrushes at six months (MD $0.02,95 \% \mathrm{Cl}-0.06$ to $0.09 ; 120$ participants (10 clusters); low heterogeneity; $\mathrm{Chi}^{2}=0.35, \mathrm{df}=1(\mathrm{P}$ $=0.56) ; I^{2}=0 \%$; Analysis 2.1). The certainty of the evidence was moderate. An RCT not included in the meta-analysis (Kelner 1963), which used a subjective assessment of gingival health and was at high risk of reporting bias, suggested a greater improvement for the electric toothbrush (53\% versus $31 \%$ improvement in gingival health).

\subsection{NRCT: gingival inflammation (short, medium and long term)}

Five NRS, with 212 participants, compared using an electric toothbrush with using a manual toothbrush on gingival inflammation levels over four weeks to 16 months (Bratel 1991; Gertenrich 1967a; Gertenrich 1967b; Gertenrich 1967c; Gertenrich 1967d).

There was no evidence of a difference between brushes in one study evaluating gingival inflammation at four weeks (Table 10). When measured at time points between eight and 34 weeks, the studies varied but provided no strong evidence of a difference in levels of gingival inflammation between the electric and manual toothbrushes (Table 11). In the long term, one NRS with participants with moderate levels of ID showed no evidence of a difference for gingival inflammation at 16 months (MD $0.00,95 \% \mathrm{Cl}-0.15$ to 0.15 ; Analysis 2.3; Table 12). The certainty of the evidence was very low. The influence of other issues such as the level of ID, who was doing the brushing and the levels of assistance provided confounded the findings.

\subsection{Plaque (short term)}

Four RCTs, with 270 participants, compared using an electric toothbrush with using a manual toothbrush, for people with ID, on plaque levels over one, two or four weeks (Christen 2007; Dôgan 2004; Kaschke 2005; Shaw 1983; Table 13). All of the studies were cross-over trials, three included a third arm that compared a special manual toothbrush (reported above). None of the studies presented data in a format that could be included in a metaanalysis. Two studies showed evidence in favour of the electric toothbrush short term; the other two studies showed no evidence of a difference between the two toothbrushes. The benefit of using an electric toothbrush versus a conventional manual toothbrush short term was unclear; the certainty of the evidence was very low.

\subsection{Plaque (medium term)}

Three RCTs (two of which were cluster RCTs), with 220 participants, compared using an electric toothbrush with using a manual toothbrush on plaque levels for people with ID, over three, four, six and 12 months (Carr 1997; Garcia-Carrillo 2016; Kelner 1963; Table 14).

Two cluster RCTs, with 120 participants, were included in a metaanalysis and did not show clear evidence of a difference between electric and manual toothbrushes at six months for people with mild or moderate ID in one study and unclear levels of ID in the other (SMD $0.29,95 \% \mathrm{Cl}-0.07$ to 0.65 ; Analysis 2.2). The certainty of the evidence was low. The RCT not included in the meta-analysis (Kelner 1963), which used a subjective assessment of oral hygiene and was at high risk of reporting bias, suggested 
a greater improvement in oral hygiene for the electric toothbrush ( $27 \%$ versus $18 \%$ improvement in oral hygiene).

\subsection{NRCT: plaque (short, medium and long term)}

Five NRS, with 193 participants, compared using an electric toothbrush with using a manual toothbrush, for people with ID, on plaque levels over four weeks to 16 months (Bratel 1991; Gertenrich 1967a; Gertenrich 1967b; Gertenrich 1967c; Gertenrich 1967d; Table 15; Table 16; Table 17).

The data provided at the longest time points by the five studies (ranging between eight and 34 weeks) were included in a metaanalysis. There was no evidence of a difference between the electric and manual toothbrushes in the medium term (SMD 0.04, 95\% Cl0.38 to 0.46 ; Analysis 2.4; moderate heterogeneity; $\mathrm{Tau}^{2}=0.10 ; \mathrm{Chi}^{2}$ $\left.=6.97, \mathrm{df}=4(\mathrm{P}=0.14) ; \mathrm{I}^{2}=43 \%\right)$. The certainty of the evidence was very low.

\subsection{Calculus (medium term)}

Three RCTs, two of which were cluster RCTs, with 220 participants, compared using an electric toothbrush with using a manual toothbrush, for people with ID, on calculus levels over a periods of four, six and 12 months (Carr 1997; Garcia-Carrillo 2016; Kelner 1963; Table 18).

Two cluster RCTs, with 120 participants (10 clusters), were included in a meta-analysis and showed no evidence of a difference for calculus levels between toothbrushes at six months (SMD -0.04, $95 \% \mathrm{Cl}-0.40$ to 0.32 ; Analysis 2.5 ; low-to-moderate heterogeneity; $\left.\mathrm{Chi}^{2}=1.44, \mathrm{df}=1(\mathrm{P}=0.23) ; \mathrm{I}^{2}=30 \%\right)$. The other RCT found in favour of the electric toothbrush. The certainty of the evidence was low.

\subsection{Changes in behaviour, attitude and self-efficacy}

One NRS assessed participant and carer experiences via a questionnaire, and found no evidence of a difference between toothbrushes for ease of use (question: "Do you find it difficult to brush your patient's teeth?") (Bratel 1991). The study reported that $10 / 12$ people with ID using the electric toothbrush reported a preference for it, finding the electric toothbrush easier to use and to have "better cleaning properties to the manual toothbrush" (Table 19). Answers to the other questions were reported narratively and only for participants in the intervention group.

\subsection{Adverse effects}

The adverse effects reported included discomfort when brushing, difficulty controlling the force and position of the electric toothbrush, and the electric toothbrush being out of order (Bratel 1991), and participants being frightened when the electric toothbrush was first used (Christen 2007; Gertenrich 1967b; Kaschke 2005). Carr 1997 reported participants being dismissed from the study, but gave no clear reason. Garcia-Carrillo 2016 looked for adverse effects by regular visual inspection and there were none. The other studies did not report adverse effects.

\section{Comparison 3: oral hygiene care training versus no oral hygiene care training or a placebo training session for carers of people with intellectual disabilities}

Four RCTs and three NRSs evaluated oral hygiene care training for carers of people with ID. See Summary of findings 3.

\subsection{Gingival inflammation (medium term)}

Two RCTs, with 99 participants, compared the effect of oral hygiene care training of formal carers with no training of formal carers, on the levels of gingival inflammation of the people with ID for whom they cared (people with mild-to-profound levels of ID in one study and severe-to-profound levels in the other study), over periods of eight weeks (Phlypo 2016) and six to nine months (Mac Giolla Phadraig 2015) (Table 20). Meta-analysis of these two studies showed no evidence of a difference in the medium term (MD -0.09, $95 \% \mathrm{Cl}-0.63$ to 0.45 ; Analysis 3.1; high heterogeneity; $\mathrm{Chi}^{2}=3.16, \mathrm{df}$ $\left.=1(P=0.08) ; I^{2}=68 \%\right)$. The certainty of the evidence was low.

\subsection{Plaque (medium term)}

Two RCTs, with 99 participants, living in residential settings, compared the effect of oral hygiene care training of formal carers with no training of formal carers, on the plaque levels of the people with ID for whom they cared (people with mild-to-profound levels of ID in one study and severe-to-profound levels in the other study), over periods of eight weeks (Phlypo 2016) and six to nine months (Mac Giolla Phadraig 2015) (Table 21). Meta-analysis of these two studies showed little or no difference between the groups in the medium term (MD $-0.07,95 \% \mathrm{Cl}-0.26$ to 0.13 ; Analysis 3.2). The certainty of the evidence was low.

\subsection{NRCT: plaque (short and medium term)}

One NRCT, with 34 participants, living in a residential setting, compared the effect of oral hygiene care training of formal carers with no training of formal carers, on the plaque levels of the people with moderate-to-profound ID for whom they cared, over 21 days (Lange 2000; Table 22). Training carers resulted in reduced plaque levels in people with ID when compared to carers who were not trained (MD $-0.69,95 \% \mathrm{Cl}-0.97$ to -0.41 ; Analysis 3.3). The NRCT reported that carers who had training with accountability showed strong evidence of lower plaque levels than the group of carers without accountability (MD $-1.89,95 \% \mathrm{Cl}-2.12$ to -1.66$)$. The certainty of the evidence was low.

One ITS study with 11 participants compared the effect of oral hygiene care training of formal carers with no training of formal carers, on plaque levels of the people with ID for whom they cared, over five months (Glassman 2006). Training of carers in oral hygiene care for people with ID may have had a positive impact on the plaque levels of the people with ID for whom they cared in the medium term (five months) if the carers were supported and encouraged to apply the training in practice subsequently (Table 23). Meta-analysis was not possible for this study due to the format of the data presented. The certainty of the evidence was very low.

\section{4 and 3.5 Knowledge of carers (short and medium term)}

Three RCTs, with 213 participants (156 of whom were in 35 clusters), compared oral hygiene care training for carers of people with ID, at follow-up of one day, eight weeks, and six to 11 months, with no training for carers or a placebo training session, on their oral care knowledge (Gonzalez 2013; Mac Giolla Phadraig 2015; Phlypo 2016; Table 24).

One RCT found no evidence that a 90-minute training session increased carer oral health knowledge in the short term (MD $0.59,95 \% \mathrm{Cl}-0.80$ to 1.98 ; Analysis 3.4). The two RCTs measuring outcomes in the medium term, which used the same assessment questionnaire and had no heterogeneity, showed a moderate effect 
on oral health knowledge in favour of training formal carers (MD $0.69,95 \% \mathrm{Cl} 0.31$ to 1.06 ; Analysis 3.5). The certainty of the evidence was low.

\subsection{Behaviour, attitude and self-efficacy of carers (short and medium term)}

Two RCTs, one of which was a cluster RCT, with 189 formal carer participants compared the effect of oral hygiene care training for carers with no training of carers on self-reported changes in behaviour, attitude and self-efficacy together, over eight weeks (Phlypo 2016) and six to 11 months (Mac Giolla Phadraig 2015). The two studies were combined in a meta-analysis and showed no evidence of a difference (MD $0.15,95 \% \mathrm{Cl}-0.80$ to 1.10 ; moderate heterogeneity; $\mathrm{Chi}^{2}=2.50, \mathrm{df}=1(\mathrm{P}=0.11) ; \mathrm{I}^{2}=60 \%$; Analysis 3.6; Table 25). The certainty of the evidence was very low.

One RCT, with 193 formal carer participants, compared the effect of oral hygiene care training for carers versus no training, on behaviour only, over four weeks, on five specific self-reported oral care behaviours (Olmos 2016; Table 26). The study reported strong evidence of positive differences for all five behaviours in the intervention group from baseline $(\mathrm{P}<0.01$; no mean or SD provided; $\%$ data only); there was no strong evidence of positive differences in the control group. Meta-analysis was not possible due to the format of the data.

Two ITS studies with 15 formal carer participants examined the effect of oral hygiene care training of carers on their oral hygiene care skills and behaviour as well as the behaviour of the people with ID for whom they cared (Glassman 2006; Kissel 1983; Table 27). One of these studies reported general increases in carers' use of training, a reduction in the level of assistance required and an "upward trend" in the level of self-initiated steps in the toothbrushing routine for the people with ID (Kissel 1983). The other study reported an increase in the caregivers' presence during the toothbrushing session, an increase in the seconds spent brushing by the people with ID and a very slight increase in the aptitude of the person with ID in relation to toothbrushing (Glassman 2006). Meta-analysis was not possible for these studies due to the format of the data presented. The certainty of the evidence was very low.

\subsection{Adverse effects}

Gonzalez 2013 assessed stress and fatigue following carer completion of a questionnaire, and found there was none. The other studies did not report adverse effects.

\section{Comparison 4: oral hygiene training versus no oral hygiene training for people with intellectual disabilities}

Two RCTs and four NRSs assessed oral hygiene training for people with ID. See Summary of findings 4.

\subsection{Gingival inflammation (short term)}

One RCT, with 10 participants, compared oral hygiene training for people with ID with no training, on gingival inflammation, over a six to seven weeks (Lange 1985). It found no evidence of a reduction in gingival inflammation due to training (MD $-0.28,95 \% \mathrm{Cl}-0.90$ to 0.34 ; Analysis 4.1; Table 28). The certainty of the evidence was low.

\subsection{Plaque (short term)}

Two RCTs, with 36 participants, compared oral hygiene training for people with ID with no training on plaque levels over five to seven weeks (Albino 1979; Lange 1985; Table 29). One study showed a moderate effect size on plaque levels in favour of oral hygiene training of people with ID in the short term (MD $-0.47,95 \% \mathrm{Cl}-0.92$ to -0.02; Analysis 4.2). The certainty of the evidence was low.

\subsection{NRCT: behaviour (medium and long term)}

Four ITS studies, with 59 participants, examined oral hygiene training for people with ID on their toothbrushing behaviour over periods of 100 days, 30 weeks and 19 months (Bouter 1979; Jarman 1983; Snell 1989). One study was unclear in relation to the duration of the intervention (Abramson 1972; Table 30). Oral hygiene training of people with ID may have impacted on their toothbrushing behaviour but it was unclear how this would impact on their oral health. Meta-analysis was not possible due to the format of the data presented. The certainty of the evidence was very low.

\subsection{Adverse effects}

Bouter 1979 reported one participant had nausea (the researchers considered it to be a delaying tactic by the participant), and Snell 1989 reported participants displaying sensitivity to mouths being held open. The other studies did not report any adverse events (Abramson 1972; Albino 1979; Jarman 1983; Lange 1985).

\section{Comparison 5: scheduled dental recall intervals $(1,3$ or 6 months) plus daily supervised toothbrushing versus usual care for people with intellectual disabilities}

One cluster-RCT, with 304 participants, compared the benefit of regular dental recalls plus daily supervised toothbrushing versus no supervision or planned dental professional intervention, for people with ID, on plaque, gingival inflammation, gingival bleeding and calculus over three, six, 12, 18 and 24 months (Shaw 1991). The recall intervals were 1-, 3- and 6- monthly: the 1-and 3-monthly recalls involved reinforcement of oral hygiene and a professional prophylaxis; the 6-monthly recall involved reinforcement of oral hygiene only. The findings at 24 months are reported here; at earlier timepoints, the number of participants was not reported (Table 31; Table 32; Table 33; Table 34). The certainty of the evidence for all reported outcomes was low. See Summary of findings 5.

\subsection{Gingival pocketing (long term)}

Meta-analysis at 24 months showed effect sizes for the Code 1 gingival pockets were moderate in favour of regular dental recalls and supervised toothbrushing over usual care (1-monthly: MD $0.60,95 \% \mathrm{Cl}-0.97$ to -0.23 ; 3-monthly: $\mathrm{MD}-0.50,95 \% \mathrm{Cl}-0.85$ to 0.15 ; 6-monthly: $\mathrm{MD}-0.40,95 \% \mathrm{Cl}-0.76$ to -0.04 ; Analysis 5.1 ).

\subsection{Gingival bleeding (long term)}

There was no evidence of a difference between regularly scheduled visits and supervised toothbrushing versus usual care for gingival bleeding at 24 months (1-monthly: $\mathrm{MD}-0.20,95 \% \mathrm{Cl}-0.86$ to 0.46 ; 3-monthly: MD $-0.10,95 \% \mathrm{Cl}-0.73$ to 0.53 ; 6-monthly: MD $0.20,95 \%$ $\mathrm{Cl}-0.38$ to 0.78 ; Analysis 5.2).

\subsection{Plaque (long term)}

Meta-analysis of data showed the effect size for plaque levels at 24 months was large in favour of regular dental recall intervals and 
supervised toothbrushing (1-monthly: $\mathrm{MD}-0.70,95 \% \mathrm{Cl}-1.30$ to 0.10 ; 3-monthly: $\mathrm{MD}-0.80,95 \% \mathrm{Cl}-1.38$ to -0.22 ; 6-monthly: $\mathrm{MD}-$ $0.70,95 \% \mathrm{Cl}-1.22$ to -0.18 ; Analysis 5.3 ).

\subsection{Calculus (long term)}

The effect size at 24 months for calculus levels was large in favour of regular dental recalls at one- and three-monthly intervals (1monthly: MD $-2.00,95 \% \mathrm{Cl}-2.64$ to $-1.36 ; 3-$ monthly: MD $-1.40,95 \%$ $\mathrm{Cl}-2.04$ to -0.76$)$, but the difference was not seen in the six-monthly interval group, which only received a scale and polish at baseline (MD $-0.10,95 \% \mathrm{Cl}-0.72$ to 0.52 ; Analysis 5.4).

\subsection{Caries}

Although the study set out to measure caries, no results were reported.

\subsection{Adverse effects}

None reported.

\section{Comparison 6: discussion of individual clinical photographs as a toothbrushing motivator versus no discussion of clinical photographs for people with intellectual disabilities}

One RCT, with 29 participants, showed people with ID photographs of their dental plaque shown up by the disclosing agent, taken at monthly oral hygiene sessions during the intervention, and discussed these with the participants in the intervention group over six months (Bickley 1990). Both groups received general oral hygiene instruction. See Summary of findings 6 .

\subsection{Plaque (medium term)}

Meta-analysis showed no evidence of a difference in plaque levels between intervention and control (MD $-0.10,95 \% \mathrm{Cl}-1.85$ to 1.65 ), or in the extrinsic stain score (MD $-2.60,95 \% \mathrm{Cl}-19.45$ to 14.25 ; Analysis 6.1 ; Table 35$)$. The certainty of the evidence was very low.

\subsection{Adverse effects}

None reported.

\section{Comparison 7: daily, twice-weekly and once-weekly} frequencies of toothbrushing versus usual care for people with intellectual disabilities

One RCT, with 80 participants, compared the frequency of brushing over 21 days on gingival inflammation (Swallow 1969). Daily brushing, carried out by dental students, was compared to twice weekly, once weekly or usual care. This study also compared the use of an electric toothbrush and a manual toothbrush (reported earlier). See Summary of findings 7 .

\subsection{Gingival inflammation (short term)}

For people with ID, who had their teeth brushed by a dental student, the more frequently the teeth were brushed the greater the reduction in gingival inflammation in the short term ( 21 days; Table 36). The certainty of the evidence was low.

\subsection{Adverse effects}

None reported.
Comparison 8: use of a toothpaste with a plaque-disclosing agent versus a conventional toothpaste for people with intellectual disabilities

One non-randomised, two-armed cross-over trial, with 40 participants aged 7 to 13 years with Down Syndrome, compared using a toothpaste containing a plaque-disclosing agent with using a conventional toothpaste on plaque and gingival inflammation levels over 10 days (Teitelbaum 2009). See Summary of findings 8.

\subsection{Plaque and gingival inflammation (short term)}

The toothpaste containing a plaque-disclosing agent when brushing may have reduced plaque and gingival inflammation in the short term (10 days) (Table 37; Table 38). The certainty of the evidence was very low.

\subsection{Adverse effects}

The study tested for an allergic reaction to any tested chemical agent; no adverse effects were reported.

Comparison 9: individualised oral care plan versus usual care (according to guidelines) for people with intellectual disabilities

One NRCT, with 79 participants, compared an individualised oral care plan with usual care, on plaque levels, over 12 months (Altabet 2003; Table 39). See Summary of findings 9.

\subsection{Plaque (medium term)}

An individualised oral care plan may have reduced plaque levels for people with ID over 12 months. The certainty of the evidence was very low.

\subsection{Adverse effects}

None reported.

\section{DISCUSSION}

\section{Summary of main results}

This review reported findings for nine comparisons of different types of oral hygiene interventions targeting people with ID or their carers. Four of the comparisons included between six and 13 studies. Only one study each assessed the remaining five comparisons. The individual studies were generally small; many studies having less than 50 participants, which resulted in most of the meta-analyses having fewer than 100 participants in total. The main outcomes reported were gingival health, oral hygiene knowledge, and oral hygiene behaviour. Dental caries and quality of life were not reported in any of the studies.

\section{Comparison 1: special manual toothbrushes versus conventional manual toothbrushes for people with intellectual disabilities (six RCTs and one NRS)}

Very low-certainty evidence from one RCT medium term (six weeks to 12 months) found a small effect size in favour of a special manual toothbrush (Superbrush) compared to a conventional manual toothbrush for reducing levels of gingival inflammation and possibly plaque. In this study, toothbrushing was carried out by formal carers for people with moderate-to-profound ID. There was no difference in effect size between the two toothbrushes for the other outcomes and time points measured. 
One NRS study compared the Collis Curve toothbrush to a conventional toothbrush used by student dental nurses for people with profound ID and found no difference between the two toothbrushes in the medium term.

\section{Comparison 2: electric toothbrushes versus manual toothbrushes for people with intellectual disabilities (eight RCTs and five NRS)}

There was moderate-certainty evidence from two RCTs in relation to the assessment of gingival inflammation in the medium term, indicating that there was little or no difference between the electric and manual toothbrushes when used by people with mild-tomoderate or unclear levels of ID, with and without some assistance from formal carers. Low-certainty evidence found no difference between toothbrush types for medium-term plaque.

The findings in the NRS were broadly similar to the findings of the RCT meta-analysis, but the certainty of the evidence was very low. The plaque and gingival inflammation levels of the control group in all of these NRS studies improved from baseline, which was credited to the daily brushing routine instigated as part of the studies.

The evidence in relation to gingival inflammation and plaque, short term, was inconclusive as the findings were inconsistent between the four studies; the data could not be included in a metaanalysis. The evidence was low- to very-low certainty. The electric toothbrushes had a variety of modes of action. The difference in findings between the types of electric toothbrush used may have been relevant.

Comparison 3: oral hygiene care training of carers versus no oral hygiene care training for carers of people with intellectual disabilities (four RCTs and three NRS)

There was low-certainty evidence that oral hygiene care training for formal carers had no beneficial effect on the level of gingival inflammation or plaque for people with mixed levels of ID for whom they cared, in the medium term, compared to no oral hygiene care training. One NRS showed strong evidence of a difference in plaque levels short term, in favour of the intervention, when the formal carers were made accountable for the level of care they provided for people with moderate-to-profound levels of ID (Table 22; Analysis 3.3).

Changes in formal carers' knowledge following training showed a moderate improvement in the medium term, but attitude, selfefficacy and behaviour of carers did not seem to improve after training, though the findings across studies were inconsistent. The certainty of RCT evidence for these outcomes was low to very low. Issues such as level of attendance at training, use of the educational resources, instruction on how to apply the training in practice and levels of support provided to carers were identified by the study authors as possibly impacting on the outcomes.

Comparison 4: oral hygiene training versus no oral hygiene training of people with intellectual disabilities (two RCTs and four NRS)

There were moderate reductions in the levels of plaque (but not gingival inflammation) in favour of oral hygiene training of people with mixed levels of ID compared to no oral hygiene training in

Oral hygiene interventions for people with intellectual disabilities (Review)

Copyright (c) 2019 The Cochrane Collaboration. Published by John Wiley \& Sons, Ltd. the short term (Table 28; Table 29; Analysis 4.1; Analysis 4.2). The certainty of the evidence was very low.

Three of the four NRS assessing changes in behaviour found in favour of the training as measured by an increase in the steps achieved in a toothbrushing routine for people with severe and profound levels of ID. The certainty of the evidence was very low (Table 30). It was unclear if the improvement in the toothbrushing routines in the NRS impacted on gingival health, as no clinical measurements were carried out.

\section{Comparison 5: one-, three- and six-monthly dental recall intervals plus daily supervised toothbrushing versus usual care for people with intellectual disabilities (one RCT)}

One RCT provided low-certainty evidence that regular dental recall visits and supervised toothrbushing might have improved gingival health (gingival pocketing, plaque and calculus levels) in the long term for people with unclear levels of ID (Table 31; Table 32; Table 33; Table 34; Analysis 5.1; Analysis 5.2; Analysis 5.3; Analysis 5.4). The difference between one-, three- and six-monthly recall intervals was minimal and unlikely to be clinically significant.

Comparison 6: discussion of individual clinical photographs as a toothbrushing motivator versus no discussion of photographs for people with intellectual disabilities (one RCT)

One RCT provided very low-certainty evidence demonstrating that the discussion of clinical photographs as an oral hygiene motivator most likely had no effect on plaque levels in the medium term (Table 35; Analysis 6.1).

\section{Comparison 7: daily, twice-weekly and once-weekly frequencies of teeth brushing versus usual care for people with intellectual disabilities (one RCT)}

One RCT provided low-certainty evidence that brushing daily compared to brushing twice weekly, once weekly or usual care may reduce plaque levels in the short term. The brushing was carried out by a dental professional in this intervention (Table 36).

\section{Comparison 8: use of a toothpaste with a plaque-disclosing agent versus a conventional toothpaste for people with intellectual disabilities (one NRS)}

One NRS found that the use of a toothpaste with a plaquedisclosing agent might reduce plaque and gingival inflammation levels for children with Down Syndrome (mild and moderate levels of ID), more than conventional toothpaste, in the short term (Table 37; Table 38). The certainty of the evidence was very low.

\section{Comparison 9: individualised oral care plan versus usual care (according to guidelines) for people with intellectual disabilities (one NRS)}

One NRS found that individualised oral care plans might improve oral hygiene for people with mixed levels of ID, in the medium term (Table 39). The intervention involved the input of a multidisciplinary team. The certainty of the evidence was very low.

\section{Adverse effects}

Only one study considered adverse effects as a formal outcome. Most studies did not report adverse effects. Some studies that reported adverse effects informally, reported transient difficulties with the electric and special manual toothbrushes but did not 
quantify them. It should be noted that any persistent resistance to clinical assessments or interventions resulted in participants being excluded from the studies.

\section{Overall completeness and applicability of evidence}

The aim of this review was to determine the effects (benefits and harms) of oral hygiene interventions for people with ID. The clinical and behavioural outcomes focused predominately on the potential benefits. Any persistent resistance to the clinical assessments or to the intervention, by people with ID, resulted in them being excluded from the study. Any harms identified were not considered as a formal outcome, often not quantified and tended to be reported in the discussion section. Any formal qualitative assessments of the interventions were completed by the carers involved and were normally in the form of poststudy interviews.

None of the studies formally addressed quality of life changes and long-term, patient-centred outcomes. There were occasional comments in the discussion section of the studies regarding increases in self-efficacy or confidence of the carers. The lack of qualitative outcomes may be linked to the age of many of the studies and lack of reporting standards. No studies provided data on dental caries. In relation to unintended effects, no studies reported any positive unintended effects. Negative unintended effects or adverse effects were reported only within the text; no formal assessments were reported.

All of the other primary and secondary outcomes outlined in our protocol were reported. The clinical outcomes reported were gingival inflammation, plaque and calculus. The tools used to assess these outcomes were modified or adapted for the ID population. Any differences observed in the clinical assessments must be considered in relation to their practical impact on gingival health long term. The non-clinical outcomes reported for the carers were knowledge, behaviour, attitude and self-efficacy. Changes in behaviour were reported for people with ID.

The topics considered for subgroup analysis, as outlined in the protocol, were recorded for the individual studies, but there were insufficient data to undertake these analyses.

\section{Validity of assessments used}

Most studies assessed clinical outcomes using validated tools, other than in six studies which used subjective assessments (Albino 1979; Gertenrich 1967a; Gertenrich 1967b; Gertenrich 1967c; Gertenrich 1967d; Kelner 1963). Many of the validated tools were modified, some to a considerable extent; some of these modifications had been validated and others had not. The level of modifications to the assessment tools highlighted the difficulties involved in carrying out clinical assessments for this population.

The use of subjective clinical assessments is most likely an historical issue and unlikely to occur in future interventions. Despite the subjective nature of these assessments, we considered these studies to add value to this review; their findings were graded with the quality of these assessments in mind.

The non-clinical outcomes (knowledge, behaviour, attitude and self-efficacy) were less well validated. The studies that included observations of behaviour were assessed for reliability. One study, using a questionnaire to assess knowledge, self-efficacy, attitude and behaviour, reported that the tool had been validated; another study used the same tool. Another study, using a questionnaire, reported getting "expert advice on the content."

\section{COM-B characteristics identified in the interventions}

We attempted to map the behavioural change elements of the interventions in this review using the COM-B system for understanding behaviour and behavioural change (Michie 2011). These elements are: capability: defined as the psychological and physical capacity to engage in the activity; opportunity: both physical and social, which considers contexts beyond the individual; and motivation: both automatic and reflexive; which might result in conscious decision-making. An intervention may change one or more of these elements resulting in the desired behaviour of performing regular oral hygiene.

\section{Capability}

The potential for increasing the physical capability of the participants was identified in interventions that focused on training, modelling or enablement in the skill of toothbrushing. The potential for increasing psychological capability was predominantly identified in interventions for people with mild or moderate ID as they could have the capability to reflect and to make the connections between any information provided and the benefit to them. Interventions targeting people with more severe levels of ID may benefit more from interventions that use incentivisation, persuasion, coercion or restriction as a psychological tool.

\section{Opportunity}

The element of opportunity to change behaviour was considered, for example, if the intervention attempted to provide additional time or resources to the oral hygiene routine (physical opportunity) or to make the oral hygiene routine socially expected or the cultural norm (social opportunity). Physical opportunity most often involved specific time being set aside for the toothbrushing routine, or environmental restructuring such as providing different oral hygiene or other aids and changing the setting where the toothbrushing took place. Social opportunities identified were monitoring or recording of the oral hygiene routine; such as a toothbrushing diary or staff monitoring. Social opportunity-based interventions tended to focus on carers rather than people with ID. One example of an intervention that did focus on children with ID, involved a toothbrushing session using a plaque-disclosing solution in a classroom setting; the children were aware of how their classmates were performing, which may act as a social cue in relation to the toothbrushing habit (Shaw 1983).

\section{Motivation}

The element of motivation to change behaviour was considered if the intervention included the potential to reflect on the benefit of changing behaviour or for the behavioural change to become an automatic process. It was the least common element identified in the interventions. Reflective motivation was identified in interventions that included some element of feedback in relation to behaviour or performance and was seen predominantly in the interventions targeting carers. No studies were identified as having the potential for the behavioural change to become automatic. This may have been due to the duration of the interventions, as the development of habit can take some time; 19 of the studies were followed up for three months or less, and only three for more than 12 months. 
To ensure that an intervention that seems effective is successfully replicated in a new setting, the explicit causal processes that elicited the behavioural change must be identified and replicated. It is difficult to identify the COM-B elements retrospectively; we can only claim that the intervention may have provided these elements. The decision to retrospectively identify these elements in our review provided valuable insight into the causal processes that were potentially in action. By mapping the behavioural change elements and intervention functions inherent in the interventions in this review, we hope we have assisted in the identification of the causal processes that should be replicated. These details are recorded in the Characteristics of included studies table.

\section{Quality of the evidence}

The certainty of the evidence varied from moderate to very low for comparisons with RCT evidence, and was very low for comparisons assessed only in NRS. The quality of reporting was poor; the lack of detail provided in relation to the methodology impacted on the assessment of risk of bias. Cross-over studies were not reported in a way that made them amenable to meta-analysis. More than half of the studies were pre-2000; before the introduction of guidelines such as the CONSORT statement in relation to the standards for reporting trials (Moher 2001). A wide variety of outcome measures was used. The limited number of studies for each comparison, all with small sample sizes, led to imprecision in the effect estimates. There were many NRSs despite the fact that RCTs are feasible for all comparisons included in this review.

Better quality evidence is required for all the comparisons identified in this review. This could be achieved by modifying the inclusion criteria or by applying positive weighting for CONSORTcompliant studies. For trials using cross-over design, the MD or data from each treatment period should be reported to allow calculation of the standard error of the SD or to approximate the correlation between results from treatment periods, so that data from these studies can be combined in any meta-analysis.

\section{Potential biases in the review process}

The most common element reducing the certainty of the evidence was the 'unclear' elements under all the risk of bias headings, most notably in relation to outcome reporting, which was predominantly as a consequence of the absence of study protocols. The level of 'high' risk in relation to randomisation was caused by the inclusion of NRSs.

We excluded 16 studies as we were unable to source the articles, unable to contact the authors for more details or the data were no longer available. These studies may have added to the review.

As the first review of this topic, we have worked hard to identify all relevant studies, but we acknowledge that some studies may not have been published, registered or presented anywhere, and we did not search every database. The study designs included in this review were wider than conventional Cochrane Reviews, but this allowed the consideration of interventions that might otherwise have been excluded. It was assumed at the protocol stage that before-and-after study designs would predominate, given the diverse nature of ID, related comorbidities, environments and level of supports available. However, this assumption was not borne out. RCTs were identified for all of the interventions except for the use of toothpaste with a plaque-disclosing agent and individualised oral care plans, and there is no reason these interventions could not be assessed in RCTs.

Given the number of studies reporting little or no difference between intervention groups compared to controls, it is unlikely that there is a publication bias for positive findings in the studies being reported.

\section{Agreements and disagreements with other studies or reviews}

\section{Special manual toothbrushes}

A systematic review by Kalf-Scholte 2018 evaluated tripleheaded toothbrushes compared to single-headed toothbrushes, in a general population ("humans without fixed orthodontic appliances"), for prevalence of plaque. Kalf-Scholte 2018 included three studies also in our review (Dôgan 2004; Kaschke 2005; Sauvetre 1995). The Kalf-Scholte 2018 review conducted some subgroup analyses that suggested that when toothbrushing was assisted or carried out by a carer, there may have been a greater benefit to using a triple-headed toothbrush (Superbrush). In our review, Bildt 2010 evaluated the impact of carer toothbrushing using a three-headed or conventional toothbrush and found a small effect size in favour of the special manual toothbrush. The Kalf-Scholte 2018 review concluded that the brushes were equally effective for self-brushers; this matched the findings of our review.

\section{Electric toothbrushes}

The findings in our review appeared to conflict with the Yaacob 2014 Cochrane Review that evaluated powered versus manual toothbrushes in the general population (people with disabilities were excluded). Yaacob 2014 concluded that electric toothbrushes were more effective than manual toothbrushes in the short (one to three months) and long (greater than three months) term. It found a moderate effect size in favour of the electric toothbrush short term (SMD $-0.50,95 \% \mathrm{Cl}-0.70$ to -0.31 ) and long term (SMD $-0.47,95 \% \mathrm{Cl}-0.82$ to -0.11 ). Our review showed no evidence of a difference between toothbrushes for gingival inflammation levels at six months (MD $0.02,95 \% \mathrm{Cl}-0.06$ to 0.09 ; Analysis 2.1).

The greatest differences in the Yaacob 2014 review were seen in the studies that compared an electric toothbrush with a rotating oscillation mode of action. There were only two studies in our review that compared an electric toothbrush with a rotation oscillation mode of action (Braun Plaque Control 3D) to a manual toothbrush, one RCT and one NRS. The RCT found strong evidence of a difference in plaque levels in favour of the electric toothbrush after one week ( $P<0.001$; Dôgan 2004). The ITS found some strong evidence of differences in subgroups for plaque at 12 weeks (those using the electric toothbrush unaided) and gingivitis at 16 months (those using the electric toothbrush unaided) in favour of the electric toothbrush ( $P<0.05$; Bratel 1991). The data from these studies were not suitable for meta-analyses.

\section{Oral hygiene care training of carers}

Comparisons in relation to changes in carer knowledge, attitude, self-efficacy and behaviour in this review could be made to studies directed at carers in long-term healthcare settings. A systematic review of the effectiveness of continuing education in long-term care settings by Aylward 2003 found many of the same issues in their review in relation to the quality of the evidence, diversity 
of assessment tools, level of attrition, applying the training in practice and levels of support provided to carers at management level. Of the studies in their review that assessed both knowledge and behaviour, almost all showed an improvement in knowledge but no change in behaviour. Most of the evaluations took place immediately after the intervention, which ranged in length from 10 minutes to 52 hours.

The clinical outcomes assessed in this comparison can be compared to the Albrecht 2016 Cochrane Review, which evaluated oral health education interventions of nurses in nursing homes, and concluded that there was no difference in plaque levels compared to usual care. The two RCTs in our review similarly found no difference in plaque or gingivitis levels. Two NRS in our review found some reduction in plaque levels when staff were made accountable for the oral hygiene care of people with ID.

\section{Oral hygiene care training for people with intellectual disabilities}

The RCTs included in our review comparing oral hygiene care training for people with ID were all short-term studies. The findings were in agreement with the systematic review by Watt 2005 in relation to oral health promotion in general, which concluded that short-term interventions may have resulted in a reduction in plaque and gingivitis levels. However, they noted in their review that there was very limited evidence supporting long-term reduction in plaque and gingival bleeding outcomes. The NRS in our review, which were medium- and long-term interventions, showed some improvement in toothbrushing routines. However, these studies had small numbers of participants and were very labour intensive. Kay 1996 commented in her review, in relation to oral health promotion that the balance between input and output must be considered.

\section{Recall intervals}

Our review found one study that showed a benefit to gingival health of regular dental recall intervals (one-, three- or six-monthly) (combined with daily supervised toothbrushing); however, the certainty of the evidence was low. One Cochrane Review investigating dental recall intervals for the general population identified only one RCT, which was insufficient to draw any conclusions; this review was published in 2013 and is currently being updated (Riley 2013). Lamont 2018 found that gingivitis was not reduced more by regularly scheduled visits for scale and polish in the general population. Evidence for the value of oral hygiene instruction at dental visits in the general population is lacking (Soldani 2018).

\section{AUTHORS' CONCLUSIONS}

\section{Implications for practice}

Although some oral hygiene interventions for people with ID show evidence of benefits, the clinical importance of these benefits is unclear. Moreover, the evidence is mainly low to very low certainty, so any changes to current practice based on this review should be made cautiously, and the advice oral care professionals give about performing oral hygiene should continue to be based on their own expertise and the needs and preferences of the individual with ID and their carers.
This review found very-low certainty evidence that a special manual toothbrush (Superbrush) may reduce levels of gingival inflammation and possibly plaque medium term more than a conventional manual toothbrush for people with intellectual disabilities (ID), when the toothbrushing is carried out by carers.

The evidence in relation to the benefit of using an electric toothbrush compared to a manual toothbrush for people with ID was inconsistent between outcomes and between studies, and the certainty of the evidence ranges from very low to moderate. The moderate-certainty evidence found no difference between the two types of toothbrush for gingival inflammation medium term.

Training carers in oral hygiene care for people with ID may increase their oral hygiene knowledge but may not have a beneficial effect on their reported behaviour, attitude and self-efficacy in relation to providing oral hygiene care, nor reduce levels of gingival inflammation or plaque in people with ID. The certainty of the evidence is low to very low.

Oral hygiene training for people with ID may have some small beneficial effects on plaque levels in the short term. The certainty of the evidence is very low.

We also identified low-certainty evidence that regular scheduled dental recall intervals, combined with daily supervised toothbrushing, may help reduce gingival inflammation and plaque in people with ID in the long term, and daily toothbrushing by dental students may reduce the plaque more effectively than once- or twice-weekly toothbrushing, in the short term. Very lowcertainty evidence suggests discussions about photographs of participants' teeth cleaned with a plaque-disclosing agent may not reduce plaque in the medium term.

Very low certainty evidence from one nonrandomised study suggested there may be a benefit to gingival health from using a toothpaste with a plaque-disclosing agent, and another nonrandomised study suggested an individualised oral care plan could be beneficial.

\section{Implications for research}

Better-quality evidence is required for all the comparisons identified in this review. Future trials might address the following issues.

Participants: the number of participants in future studies needs to be increased so that the uncertainties in the review findings can be resolved. The level of ID should be clearly defined using the most up-to-date classification. The trials should focus on one level of ID or differentiate between levels of ID when reporting the findings.

Interventions: interventions targeting training of carers and people with ID might consider some alternative outcomes and tools to more carefully assess some of the behavioural changes that might occur following training. The use of the COM-B system to identify and reflect on the contexts and mechanisms of action at play in future interventions may be useful in understanding what makes an intervention effective.

Comparisons: different types of electric or special manual toothbrushes with different modes of action should be considered for comparison. 
Outcomes: agreement in relation to the ideal index to use for the various clinical outcomes should be reached to allow valid comparisons between studies, that is, a 'core outcome set', as recommended by the COMET (Core Outcome Measures in Effectiveness Trials) initiative (Williamson 2017). The level of modification of the indices should be kept to a minimum, ideally trials should use only those that have been validated. Outcomes should include dental caries, quality of life changes or long-term, patient-centred outcomes; the length of follow-up would thus need to be extended in many instances. Long-term follow-up is important for all outcomes to show if any short- and mediumterm benefits are sustained. Poor oral hygiene impacts on oral health and consequently on quality of life; it is essential that future studies assess this outcome. For trials using cross-over design, the MD or data from each treatment period should be reported to allow calculation of the standard error of the mean difference or to approximate the correlation between results from treatment periods, so that data from these studies can be combined in any meta-analysis.

Risk of bias: trial registration and improved reporting of the trials would reduce the number of 'unclear' risk of bias assessments found in this review.

\section{ACK N O WLEDGEMENTS}

We acknowledge the help of Cochrane Oral Health in the production of this protocol and review, particularly Laura MacDonald, Anne Littlewood, Janet Lear, Professor Helen Worthington, Professor Jan Clarkson, Dr Tanya Walsh and Dr Martin McCabe. We also acknowledge the peer reviewers who provided comments on the protocol or review, or both: Professor Bob Gates, Dr Deborah Kinnear, Professor Tim Newton and Dr Debbie Bonetti.

We acknowledge the considerable assistance of the library staff in the Dublin Dental University Hospital (Isabel Fleischmann, Caroline Meehan, Fleur Finlay) in sourcing articles from our own library, Trinity College Library and via inter library loans. We also thank Denis Murphy, Maria Perno Goldie, Ioannis Polyzois and Yvonne Nyblom for their assistance in sourcing particular articles. We appreciate the time and effort made by authors of included and excluded studies to source data and provide additional information.

We are very grateful to the following for their assistance in selecting studies for inclusion by translating those written in languages other than English: Margriet E van Barr, Ralph Moehler, Anette Bluemle, Darius Sagheri, Isabel Fleischmann, Maddalena Manfredi, Toru Naito, Eva Fetscher, Jörn Klein, Hilde Aga, Monica Håland and Monica Ballesteros. 


\section{RE F E R E N C E S}

\section{References to studies included in this review}

\section{Abramson 1972 \{published data only\}}

Abramson EE, Wunderlich RA. Dental hygiene training for retardates: an application of behavioral techniques. Mental Retardation 1972;10(3):6-8.

\section{Albino 1979 \{published data only\}}

* Albino JE, Schwartz BH, Goldberg HJ, Stern ME. Results of an oral hygiene program for severely retarded children. ASDC Journal of Dentistry for Children 1979;46(1):25-8.

Schwartz BH, Albino JE, Bissell GD. Development and evaluation of a dental health training program for severely retarded children. Journal of Dentistry for the Handicapped 1978;4(1):17-22.

\section{Altabet 2003 \{published data only\}}

Altabet S, Rogers K, Imes E, Boatman IM, Moncier J. Comprehensive approach toward improving oral hygiene at a state residential facility for people with mental retardation. Mental Retardation 2003;41(6):440-5.

\section{Bickley 1990 \{published data only\}}

Bickley SR, Shaw L, Shaw MJ. Use and effect of clinical photographic records on the motivation and oral hygiene practices of a group of mentally handicapped adults. Dental Health 1990;29(1):3-6.

\section{Bildt 2010 \{published data only\}}

Bildt MM, Ekhart GJ. The effect of a three-headed toothbrush on oral health in patients with severe disabilities. Journal of Disability and Oral Health 2010;11(3):120; Poster Abstract No. 208.

Ekhart GJ. Dental Care of Disabled Patients by their Caregivers; an Impact Study on the Use of a Three Head Toothbrush [Gebitsverzorging van gehandicapte patiënten door hun verzorgers; een effectstudie naar het gebruik van een driekopstandenborste]. [Masters Thesis]. Groningen: University of Groningen University Medical Center. Groningen: University of Groningen University Medical Center Groningen, 2010.

\section{Bouter 1979 \{published data only\}}

Bouter HP, Smeets PM. Teaching toothbrushing behaviour in severely retarded adults: systematic reduction of feedback and duration training. International Journal of Rehabilitation Research 1979;2(1):61-9.

\section{Bratel 1991 \{published data only\}}

* Bratel J, Berggren U. Long-term oral effects of manual or electric toothbrushes used by mentally handicapped adults. Clinical Preventive Dentistry 1991;13(4):5-7.

Bratel J, Berggren U, Hirsch JM. Electric or manual toothbrush? A comparison of the effects on the oral health of mentally handicapped adults. Clinical Preventive Dentistry 1988;10(3):23-6.
Carr 1997 \{published data only\}

Carr MP, Sterling ES, Bauchmoyer SM. Comparison of the Interplak and manual toothbrushes in a population with mental retardation/developmental disabilities (MR/DD). Special Care in Dentistry 1997;17(4):133-6.

\section{Christen 2007 \{published data only\}}

Christen S. A Clinical Investigation of a Comparison Between the Brushing Effectiveness and Manageability of a Three-Head Electric Toothbrush, a Three-Headed Manual Toothbrush and a Conventional Manual Toothbrush in Patients with Disabilities [Klinische Untersuchung der Putzeffektivität und Handhabbarkeit einer elektrischen Dreikopfzahnbürste, einer dreiköpfigen Handzahnbürste sowie einer konventionellen Handzahnbürste im Vergleich bei Patienten mit Behinderungen]. [Thesis]. Berlin: University Medicine Berlin. Berlin, 2017.

\section{Dôgan 2004 \{published data only\}}

Dôgan MC, Alacam A, Asici N, Odabas M, Seydaoglu G. Clinical evaluation of the plaque-removing ability of three different toothbrushes in a mentally disabled group. Acta Odontologica Scandinavica 2004;62(6):350-4

\section{Ferozali 2007 \{published data only\}}

Ferozali F, Johnson G, Cavagnaro A. Health benefits and reductions in bacteria from enhanced oral care. Special Care in Dentistry 2007;27(5):168-76.

\section{Garcia-Carrillo 2016 \{published data only\}}

Garcia-Carrillo A, Jover A, Pla R, Martorell A, Sota C, Gomez-Moreno G, et al. Manual versus sonic powered toothbrushing in patients with intellectual disability: a clusterrandomized clinical trial. Journal of Clinical Periodontology 2016;43(8):684-93.

ISRCTN16336355. Manual versus sonic powered tooth brushing in patients with intellectual disability (Cepillado manual versus cepillado eléctrico para la salud oral en pacientes con discapacidad intelectual leve y moderada). www.isrctn.com/ ISRCTN16336355 (first received 5 July 2015).

\section{Gertenrich 1967a \{published data only\}}

Gertenrich RL, Lewis MJ. A study of automatic and hand tooth brushing as used on retarded or handicapped patients Phase III. Journal of Dentistry for Children 1967;34(3):155-60.

\section{Gertenrich 1967b \{published data only\}}

Gertenrich RL, Lewis MJ. A study of automatic and hand tooth brushing as used on retarded or handicapped patients Phase I. Journal of Dentistry for Children 1967;34(3):145-50.

\section{Gertenrich 1967c \{published data only\}}

Gertenrich RL, Lewis MJ. A study of automatic and hand tooth brushing as used on retarded or handicapped patients Phase II. Journal of Dentistry for Children 1967;34(3):150-5. 


\section{Gertenrich 1967d \{published data only\}}

Gertenrich RL, Lewis MJ. A study of automatic and hand tooth brushing as used on retarded or handicapped patients Phase IV. Journal of Dentistry for Children 1967;34(3):161-4.

\section{Glassman 2006 \{published data only\}}

Glassman P, Miller CE. Effect of preventive dentistry training program for caregivers in community facilities on caregiver and client behavior and client oral hygiene. New York State Dental Journal 2006;72(2):38-46.

\section{Gonzalez 2013 \{published data only\}}

Gonzalez EE, Nathe CN, Logothetis DD, Pizanis VG, SanchezDils E. Training caregivers: disabilities and dental hygiene. International Journal of Dental Hygiene 2013;11(4):293-7.

\section{Jarman 1983 \{published data only\}}

Jarman PH, Iwata BA, Lorentzson AM. Development of morning self-care routines in multiply handicapped persons. Applied Research in Mental Retardation 1983;4(2):113-22.

Kaschke 2005 \{published and unpublished data\}

* Kaschke I, Klaus-Roland J, Zeller A. The effectiveness of different toothbrushes for people with special needs. Journal of Disability and Oral Health 2005;6(2):65-71.

Zeller A. Clinical Investigation of the Brushing Effectiveness and Manageability of an Electronic Toothbrush, a Three-Headed Manual Toothbrush and a Conventional Manual Toothbrush in Patients with Disabilities [Klinische Untersuchung der Putzeffektivität und Handhabbarkeit einer elekronischen Zahnbürste, einer dreiköpfigen Handzahnbürste und einer konventionellen Handzahnbürste bei Patienten mit Behinderungen]. [Masters Thesis]. Berlin: Freie Universität Berlin. Berlin: Freie Universität Berlin, 2004.

\section{Kelner 1963 \{published data only\}}

Kelner M. Comparative analysis of the effect of automatic and conventional toothbrushing in mental retardates. Pennsylvania Dental Journal 1963;30(4):102-9.

\section{Kissel 1983 \{published data only\}}

Kissel RC, Whitman TL, Reid DH. An institutional staff training and self-management program for developing multiple selfcare skills in severely/profoundly retarded individuals. Journal of Applied Behavior Analysis 1983;16(4):395-415.

\section{Lange 1985 \{published data only\}}

* Lange B. Effects of modeling on the oral health care of persons with mentally handicapping conditions. Special Care in Dentistry 1985;5(6):255-60.

Lange BM. Effects of modeling on the oral health care of mentally retarded. Dissertation Abstracts International 1981;41(11-A):4654.

\section{Lange 2000 \{published data only\}}

Lange B, Cook C, Dunning D, Froeschle ML, Kent D. Improving the oral hygiene of institutionalized mentally retarded clients. Journal of Dental Hygiene 2000;74(3):205-9.
Mac Giolla Phadraig 2015 \{published data only\}

* Mac Giolla Phadraig C, Guerin S, Nunn J. Should we educate care staff to improve the oral health and oral hygiene of people with intellectual disability in residential care? Real world lessons from a randomized controlled trial. Special Care in Dentistry 2015;35(3):92-8.

Mac Giolla Phadraig C, Guerin S, Nunn J. Train the trainer? A randomized controlled trial of a multi-tiered oral health education programme in community-based residential services for adults with intellectual disability. Community Dentistry and Oral Epidemiology 2013;41(2):182-92.

Olmos 2016 \{published data only\}

Kaschke I. Oral Health Promotion for Adult Residents of Disabled Facilities; Evaluation of the Berlin Model Project [Mundgesundheitsförderung für erwachsene Bewohner von Behinderteneinrichtungen; Evaluation des Berliner Modellprojektes]. [Masters Thesis]. Berlin: Freie Universität Berlin 2008.

Olmos I. Oral Health Promotion in Adult Handicapped Persons in Disabled People's Facilities; Evaluation of the Sustainability of the Berlin Model Project [Mundgesundheitsförderung bei erwachsenen Bewohnern mit Behinderung in Behinderteneinrichtungen; Evaluation der Nachhaltigkeit des Berliner Modellprojektes]. [Masters Thesis]. Berlin: Freie Universität Berlin. Berlin: Freie Universität Berlin, 2015.

* Olmos I, Kaschke I. Evaluation of the Berlin Oral health programme for adult residents of disabled facilities [Evalution des Berliner Mundgesundheitsprogramms für erwachsene Bewohner von Behinderteneinrichtungen]. Prophylaxe Impuls 2016;20:6-13.

\section{Phlypo 2016 \{published data only\}}

Phlypo I. Integrating community service-learning in undergraduate dental education: an oral health project in a residential facility for people with intellectual disabilities. Unpublished article.

* Phlypo I. Pilot study on the effects of oral health education for caregivers in a residential setting for people with intellectual disabilities: a randomized controlled trial. International Association of Disability and Oral Health 2016;36(3):146.

Phlypo I, De Tobel J, Marks L, De Visschere L, Koole S. Integrating community service learning in undergraduate dental education: a controlled trial in a residential facility for people with intellectual disabilities. Special Care in Dentistry 2018;38(4):201-7.

\section{Sauvetre 1995 \{published data only\}}

Sauvetre E, Rozow A, De Meel H, Richebe A, Abi-Khalil M, Demeure F. Comparison of the clinical effectiveness of a single and a triple-headed toothbrushes in a population of mentally retarded patients. Bulletin du Groupement International pour la Recherche Scientifique en Stomatologie \& Odontologie 1995;38(3-4):115-9. 
Shaw 1983 \{published data only\}

Shaw L, Harris BM, Maclaurin ET, Foster TD. Oral hygiene in handicapped children: a comparison of effectiveness in the unaided use of manual and electric toothbrushes. Dental Health 1983;22(1):4-5.

\section{Shaw 1991 \{published data only\}}

Shaw L, Shaw MJ. The effectiveness of differing dental health education programmes in improving the oral health of adults with mental handicaps. Journal of Dental Research 1990;69(4):973.

* Shaw MJ, Shaw L. The effectiveness of differing dental health education programmes in improving the oral health of adults with mental handicaps attending Birmingham adult training centres. Community Dental Health 1991;8(2):139-45.

Shaw MJ, Shaw L, Foster TD. The oral health in different groups of adults with mental handicaps attending Birmingham (UK) adult training centres. Community Dental Health 1990;7(2):135-41.

\section{Snell 1989 \{published data only\}}

Snell ME, Patrice A, Houghton A. Acquisition and maintenance of toothbrushing skills by students with cerebral palsy and mental retardation. Journal of the Association for Persons with Severe Handicaps 1989;14(3):216-26.

\section{Swallow 1969 \{published data only\}}

Swallow JN, Davies DE, Hawkins SD. Gingival disease prevalence in mentally handicapped adults. The effects of an oral hygiene programme. British Dental Journal 1969;127(8):376-9.

\section{Teitelbaum 2009 \{published data only\}}

Teitelbaum AP, Pochapski MT, Jansen JL, Sabbagh-Haddad A, Santos FA, Czlusniak GD. Evaluation of the mechanical and chemical control of dental biofilm in patients with Down syndrome. Community Dentistry and Oral Epidemiology 2009;37(5):463-7.

\section{Williams 1988 \{published data only\}}

Williams NJ, Schuman NJ. The curved-bristle toothbrush: an aid for the handicapped population. Journal of Dentistry for Children 1988;55(4):291-3.

\section{References to studies excluded from this review}

\section{Andrews 1990 \{published data only\}}

Andrews BA, Barrie RB. Effectiveness of toothbrushes and dental roulettes in mentally handicapped patients. Journal of Dental Research 1990;69(4):1084.

\section{Badra 1973 \{published data only\}}

Badra A. Dental care for the difficult child]. [Portuguese. Tribuna Odontologica do Sindicato Dos Odontologistas do Estado da Guanabara 1973;5(21):3-4.
Borglin 1969 \{published data only\}

Borglin K. Oral hygiene experiment with the dentifrice "Clean Mouth" at Vipeholm hospital [Swedish]. Apollonia 1969;17(2):43-4.

Brody 1975 \{published data only\}

Brody JF, Esslinger S, Casselman G, McGlinchey M, Mitala R. The itinerant training team: variations on a familiar concept. Mental Retardation 1975;13(2):38-42.

Bui 2003 \{published data only\}

Bui 2003. Trying to contact authors of research carried out in your university [personal communication]. Email to: A Scott 7 September 2017.

Bui C, Scott A. Powered versus conventional plaque removal in children with visual, hearing and/or intellectual disabilities. (ADRF Abstract). Australian Dental Journal 2003;48(4 ADRF Special Research Suppl):S3.

Favell 1975 \{published data only\}

Favell JE, Favell JE. Experimental analysis of a self-help training program for severely retarded boys. Research \& the Retarded 1975;2(1):6-17.

Waldron C. Contact details for Dr Judith Favell [personal communication]. Email to: Celeste Foundation 7 September 2017.

\section{Gertenrich 1972 \{published data only\}}

Gertenrich 1972. Details of your study "Utilization of the oral hygiene team in a mental health institution" [personal communication]. Email to: RL Gertenrich 25 September 2017.

Gertenrich RL, Hart RW. Utilization of the oral hygiene team in a mental health institution. Journal of Dentistry for Children 1972;39(3):174-7.

\section{Haran 2014 \{published data only\}}

Haran $\mathrm{MH}$, Dougall A. Toothbrushing training programme for adolescents with intellectual disabilities. Journal of Disability and Oral Health 2014;15(3):89.

Waldron C. Details of a study you presented at IADH 2014 [personal communication]. Email to: M Haran 18 October 2017.

\section{Horner 1975 \{published data only\}}

Horner RD, Keilitz I. Training mentally retarded adolescents to brush their teeth. Journal of Applied Behavior Analysis 1975;8(3):301-9.

ISRCTN10044161 \{published data only (unpublished sought but not used)\}

ISRCTN10044161. An investigation into a measure of oral cleanliness and its usefulness in motivating children towards improved oral hygiene. www.isrctn.com/ISRCTN10044161 (first received 28 September 2007).

Waldron C. Details of a Masters Thesis by Peter Crawford [personal communication]. Email to: 1) P Crawford and 2) University Hospital Bristol 21 August 2017. 


\section{Kaschke 2008 \{published data only\}}

Kaschke I. Oral Health Promotion for Adult Residents of Disabled Facilities - Evaluation of the Berlin Model Project [Mundgesundheitsförderung für erwachsene Bewohner von Behinderteneinrichtungen - Evaluation des Berliner Modellprojektes]. [Masters Thesis]. Berlin: Freie Universität Berlin. Berlin: Freie Universität Berlin., 2008.

Waldron C. Search of oral hygiene interventions for people with intellectual disabilities for a Cochrane Review [personal communication]. Email to: I Kaschke 2 July 2017.

\section{Lesmana 2014 \{published data only\}}

Lesmana S, Ananta E, Darwita RR. Dental health education for children with intellectual disability. Journal of Disability \& Oral Health 2014;15(3):102.

Waldron C. Details of a study presented at IADH 2014 [personal communication]. Email to: S Lesmana 18 October 2017.

\section{Lopez 1994 \{published data only\}}

Lopez J, Rosello X. Multicenter study in 80 mentally handicapped. The value of a right toothbrush. Avances en Odontoestomatologia 1994;10(7):485-8.

\section{Meador 1979 \{published data only\}}

Meador HG. Toothbrushing: a sensible approach for the mentally retarded. Dental Hygiene 1979;53(10):462-4.

\section{NCT03234231 \{published data only\}}

NCT03234231. Supervised brushing programme for intellectual disabled students. clinicaltrials.gov/show/NCT03234231 (first received 31 July 2017).

\section{NCT03791385 \{published data only\}}

NCT03791385. Effectiveness of picture exchange communication system on gingival health of children with autism spectrum disorders. clinicaltrials.gov/ct2/show/ NCT03791385 (first received 1 January 2019).

\section{Ojeda 2010 \{published data only\}}

Ojeda RP, Naranjo C. Plaque removal using manual/powered toothbrush in down syndrome patients. IADR General Session; 2010, Jul 14-17; Barcelona, Spain. Poster No: 4232. 2010.

Waldron C. Request for contact with authors of a study; Ojeda, R. P. and Naranjo, C [personal communication]. Email to: B Salinas 8 September 2017.

\section{Ribeiro 2011 \{published data only\}}

Ribeiro L, Chibinski AC, Czlusniak G, Wambier D. Chlorhexidine for cariogenic microorganisms control in children with special needs [abstract]. Proceedings of the 89th General Session of the International Association for Dental Research; 2011, Mar 16-19; San Diego (CA). Abstract no: 7442011.

\section{Schmidt 1981 \{published data only\}}

Schmidt SM, Leach M, Nicolaci AB, Sutton RB, O'Donnell JP. The dental health educator and programs for institutions with persons who are mentally retarded. Special Care in Dentistry 1981;1(4):174-8.
Waldron C. Details of a study you co-authored in 1981 [personal communication]. Email to: JP O'Donnell 19 October 2017.

Thornton 1991 \{published data only\}

Thornton J, Jones C, Gibson A, Aponte-Merced L. Effectiveness of oral hygiene instructions on low educable mentally retarded adults (IADR Abstract 1991). Journal of Dental Research 1991;70(Special Issue/Abstracts):303 (Abs 303).

Waldron C. Details of a study you presented at IADR 1991 [personal communication]. Email to: JB Thornton 17 October 2017.

\section{Zaksek 2014 \{published data only\}}

Zaksek R. Implementation of an oral health intervention for adults with intellectual and developmental disabilities. [Masters Thesis]. Louisville (KY): University of Louisville 2014.

\section{Zhou 2019 \{published data only\}}

Zhou N, Wong HM, McGrath C. Effectiveness of a visual-verbal integration model in training parents and their preschool children with intellectual and developmental disabilities to dispense a pea-sized amount of fluoridated toothpaste. Journal of Applied Research in Intellectual Disabilities 2019;32(3):657-65.

\section{References to studies awaiting assessment}

Akgun 2012 \{published data only\}

Akgun E, Yoldas O, Dogan C. Oral health status of children with mental disability: two year follow-up. Journal of Disability and Oral Health 2012;13(3):118.

\section{Birani 2008 \{published data only\}}

Birani J, Santos FS, Scannavino FLF, Martins AT. Educational video on buccal health for care-takers of patients with special needs. Journal of Disability and Oral Health 2008;9:198.

Saptiwi 2018 \{published data only\}

Saptiwi B, Sukini S, Ekoningtyas EA, Rajiani I. The application of Irene's donuts innovative school program towards the oral health care and the hygiene index of children with special needs. Indian Journal of Public Health Research and Development 2018;9(10):112-5.

\section{References to ongoing studies}

NCT03522337 \{published data only\}

NCT03522337. Effectiveness of visual pedagogy-assisted tooth-brushing training among preschoolers with special needs for oral health promotion. clinicaltrials.gov/ct2/show/ NCT03522337 (first received 11 May 2018).

\section{Additional references}

\section{Agnihotry 2016}

Agnihotry A, Fedorowicz Z, Worthington HV, Manheimer E, Stevenson RG. Systematic reviews in oral health: a quality imperative. Journal of Evidence-based Medicine 2016;9:47-52. [DOI: 10.1111/jebm.12189; PUBMED: 26845573] 


\section{Ainamo 1975}

Ainamo J, Bay I. Problems and proposals for recording gingivitis and plaque. International Dental Journal 1975;25(4):229-35.

\section{Al Kindi 2016}

Al Kindi NA, Nunn J. The use of the BDA Case Mix Model to assess the need for referral of patients to specialist dental services. British Dental Journal 2016;220(8):401-6.

\section{Albrecht 2016}

Albrecht M, Kupfer R, Reissmann DR, Mühlhauser I, Köpke S. Oral health educational interventions for nursing home staff and residents. Cochrane Database of Systematic Reviews 2016, Issue 9. [DOI: 10.1002/14651858.CD010535.pub2]

\section{Anders 2010}

Anders PL, Davis EL. Oral health of patients with intellectual disabilities: a systematic review. Special Care in Dentistry 2010;30(3):110-7.

\section{Axelsson 1978}

Axelsson P, Lindhe J. Effect of controlled oral hygiene procedures on caries and periodontal disease in adults. Journal of Clinical Periodontology 1978;5(2):133-51.

\section{Axelsson 2004}

Axelsson P, Nystrom B, Lindhe J. The long-term effect of a plaque control program on tooth mortality, caries and periodontal disease in adults. Results after 30 years of maintenance. Journal of Clinical Periodontology 2004;31(9):749-57.

\section{Aylward 2003}

Aylward S, Stolee P, Keat N, Johncox V. Effectiveness of continuing education in long-term care: a literature review. Gerontologist 2003;43(2):259-71.

\section{Beck 1993}

Beck S, Yasko JM. Guidelines for Oral Care. 2nd Edition. Vol. 1-3, Cary (IL): Sage Products, 1993.

\section{Bender 2008}

Bender R, Bunce C, Clarke M, Gates S, Lange S, Pace NL, et al. Attention should be given to multiplicity issues in systematic reviews. Journal of Clinical Epidemiology 2008;61(9):857-65.

\section{Bentley 1995}

Bentley CD, Disney JA. A comparison of partial and full mouth scoring of plaque and gingivitis in oral hygiene studies. Journal of Clinical Periodontology 1995;22(2):131-5.

\section{Bonetti 2015}

Bonetti D, Hampson V, Queen K, Kirk D, Clarkson J, Young L. Improving oral hygiene for patients. Nursing Standard 2015;29(19):44-50.

\section{Borgnakke 2015}

Borgnakke WS. Does treatment of periodontal disease influence systemic disease?. Dental Clinics of North America 2015;59(4):885-917.

\section{Broadbent 2011}

Broadbent JM, Thomson WM, Boyens JV, Poulton R. Dental plaque and oral health during the first 32 years of life. Journal of the American Dental Association 2011;142(4):415-26.

\section{Campanaro 2014}

Campanaro M, Huebner CE, Davis BE. Facilitators and barriers to twice daily tooth brushing among children with special health care needs. Special Care in Dentistry 2014;34(4):185-92.

\section{Campbell 2012}

Campbell MK, Piaggio G, Elbourne DR, Altman DG. CONSORT 2010 statement: extension to cluster randomised trials. BMJ 2012;345:e5661.

\section{Cane 2015}

Cane J, Richardson M, Johnston M, Ladha R, Michie S. From lists of behaviour change techniques (BCTs) to structured hierarchies: comparison of two methods of developing a hierarchy of BCTs. British Journal of Health Psychology 2015;20(1):130-50.

\section{Catteau 2011}

Catteau C, Mishellany A, Faulks D, Lassauzay C, Hennequin M. Oral health status of persons with disabilities attending special establishments. First results of the national health education programme "Oral Health and Autonomy". Bulletin du Groupèment International Pour La Recherche Scientifique en Stomatologie \& Odontologie 2011;50(1):15-8.

\section{Chadwick 2009}

Chadwick DD, Jolliffe J. A descriptive investigation of dysphagia in adults with intellectual disabilities. Journal of Intellectual Disability Research 2009;53(1):29-43.

\section{Chestnutt 1998}

Chestnutt IG, Schafer F, Jacobson AP, Stephen KW. The influence of toothbrushing frequency and post-brushing rinsing on caries experience in a caries clinical trial. Community Dentistry and Oral Epidemiology 1998;26(6):406-11.

\section{Cochrane 2018}

Cochrane Collaboration. Cochrane Glossary. www.community.cochrane.org/glossary (accessed 26 June 2018).

\section{Cochrane Oral Health 2019}

Cochrane Oral Health. Registered titles, protocols and published reviews. www.oralhealth.cochrane.org/oral-health-evidence (accessed 19 March 2019).

\section{Cooper 2013}

Cooper AM, O'Malley LA, Elison SN, Armstrong R, Burnside G, Adair $P$, et al. Primary school-based behavioural interventions for preventing caries. Cochrane Database of Systematic Reviews 2013, Issue 5. [DOI: 10.1002/14651858.CD009378.pub2]

\section{Cooper 2014}

Cooper AM, Coffey M, Dugdill L. Challenges in designing, conducting, and reporting oral health behavioral intervention 
studies in primary school age children: methodological issues. Pragmatic and Observational Research 2014;5:43-51.

\section{Covidence 2018 [Computer program]}

Veritas Health Innovation. Covidence Systematic Review Software. Melbourne, Australia: Veritas Health Innovation, 2018.

\section{Crowley 2005}

Crowley E, Whelton H, Murphy A, Kelleher K, Cronin M, Flannery $\mathrm{E}$, et al. Oral health of adults with an intellectual disability in residential care in Ireland 2003. Department of Health and Children, Dublin; 2005.

\section{De Jongh 2008}

De Jongh A, Van Houtem C, Van der Schoof M, Resida G, Broers D. Oral health status, treatment needs, and obstacles to dental care among noninstitutionalized children with severe mental disabilities in the Netherlands. Special Care in Dentistry 2008;28(3):111-5.

\section{Deeks 2011}

Deeks JJ, Higgins JP, Altman DG. Chapter 9: Analysing data and undertaking meta-analyses. In: Higgins JP, Green S, editor(s). Cochrane Handbook for Systematic Reviews of Interventions Version 5.1.0 (updated March 2011). The Cochrane Collaboration, 2011. Available from handbook.cochrane.org.

\section{Dougall 2008}

Dougall A, Fiske J. Access to special care dentistry, part 1. Access. British Dental Journal 2008;204(11):605-16.

\section{Dwan 2014}

Dwan K, Altman DG, Clarke M, Gamble C, Higgins JP, Sterne JA, et al. Evidence for the selective reporting of analyses and discrepancies in clinical trials: a systematic review of cohort studies of clinical trials. PLoS Medicine 2014;11(6):e1001666. [DOI: 10.1371/journal.pmed.1001666]

\section{Egelberg 1994}

Egelberg J, Claffey N. Periodontal Re-evaluation the Scientific Way. Copenhagen: Munksgaard, 1994.

\section{EPOC 2016}

Effective Practice, Organisation of Care. Suggested risk of bias criteria for EPOC reviews. EPOC Resources for Review Authors. Oslo: Norwegian Knowledge Centre for the Health Services, 2016.

\section{EPOC 2017}

Cochrane Effective Practice, Organisation of Care (EPOC). What study designs can be considered for inclusion in an EPOC review and what should they be called?. Available at: epoc.cochrane.org/resources/epoc-resources-review-authors. EPOC Resources for review authors, 2017.

\section{Faulks 2000}

Faulks D, Hennequin M. Evaluation of a long-term oral health program by carers of children and adults with intellectual disabilities. Special Care in Dentistry 2000;20(5):199-208.

\section{Fleiss 1987}

Fleiss JL, Park MH, Chilton NW, Alman JE, Feldman RS, $\mathrm{HH}$ Chauncey. Representativeness of the "Ramfjord teeth" for epidemiologic studies of gingivitis and periodontitis. Community Dentistry and Oral Epidemiology 1987;15:221-4.

\section{Garmyn 1998}

Garmyn P, Van Steenberghe D, Quirynen M. Efficacy of plaque control in the maintenance of gingival health: plaque control in primary and secondary prevention. Proceedings of the European Workshop on Mechanical Plaque Control; 199805 09-12; Switzerland. Berlin: Quintessence Publishing Company Inc., 1998:107-20.

\section{Glassman 2003}

Glassman P, Miller CE. Preventing dental disease for people with special needs: the need for practical preventive protocols for use in community settings. Special Care in Dentistry 2003;23(5):165-7.

\section{Glassman 2009}

Glassman P, Miller C. Social supports and prevention strategies as adjuncts and alternatives to sedation and anesthesia for people with special needs. Special Care in Dentistry 2009;29(1):31-8.

\section{Gordon 1998}

Gordon SM, Dionne RA, Snyder J. Dental fear and anxiety as a barrier to accessing oral health care among patients with special health care needs. Special Care in Dentistry 1998;18(2):88-92.

\section{Greene 1960}

Greene JG, Vermillion JR. The oral hygiene index: a method for classifying oral hygiene status. Journal of the American Dental Association 1960;61(2):172-9.

\section{Hennequin 2008}

Hennequin M, Moysan V, Jourdan D, Dorin M, Nicolas E. Inequalities in oral health for children with disabilities: a French national survey in special schools. PloS One 2008;3(6):e2564.

\section{Higgins 2011a}

Higgins JP, Altman DG, Sterne JA. Chapter 8: Assessing risk of bias in included studies. In: Higgins JP, Green S, editor(s). Cochrane Handbook for Systematic Reviews of Interventions Version 5.1.0 (updated March 2011). The Cochrane Collaboration, 2011. Available from handbook.cochrane.org.

\section{Higgins 2011b}

Higgins JP, Deeks JJ, Altman DG. Chapter 16: Special topics in statistics. In: Higgins JP, Green S, editor(s). Cochrane Handbook for Systematic Reviews of Interventions Version 5.1.0 (updated March 2011). The Cochrane Collaboration, 2011. Available from handbook.cochrane.org.

\section{Hopewell 2007}

Hopewell S, Clarke MJ, Stewart L, Tierney J. Time to publication for results of clinical trials. Cochrane Database of Systematic Reviews 2007, Issue 2. [DOI: 10.1002/14651858.MR000011.pub2] 


\section{Hopewell 2009}

Hopewell S, Loudon K, Clarke MJ, Oxman AD, Dickersin K. Publication bias in clinical trials due to statistical significance or direction of trial results. Cochrane Database of Systematic Reviews 2009, Issue 1. [DOI: 10.1002/14651858.MR000006.pub3]

\section{Horsley 2011}

Horsley T, Dingwall O, Sampson M. Checking reference lists to find additional studies for systematic reviews. Cochrane Database of Systematic Reviews 2011, Issue 8. [DOI: 10.1002/14651858.MR000026.pub2]

\section{Horwitz 2000}

Horwitz SM, Kerker BD, Owens PL, Zigler E. The health status and needs of individuals with mental retardation. New Haven (CT): Yale University School of Medicine and Special Olympics Inc. 2000.

\section{Hujoel 2005}

Hujoel PP, Cunha-Cruz J, Loesche WJ, Robertson PB. Personal oral hygiene and chronic periodontitis: a systematic review. Periodontology 2000 2005;37:29-34.

\section{Kalf-Scholte 2018}

Kalf-Scholte SM, Van der Weijden GA, Bakker EW, Slot DE. Plaque removal with triple-headed vs single-headed manual toothbrushes; a systematic review. International Journal of Dental Hygiene 2018;16(1):13-23.

\section{Kay 1996}

Kay E, Locker D. Is dental health education effective? A systematic review of current evidence. Community Dentistry and Oral Epidemiology 1996;24(4):231-5.

\section{Kaye 2005}

Kaye PL, Fiske J, Bower EJ, Newton JT, Fenlon M. Views and experiences of parents and siblings of adults with Down syndrome regarding oral healthcare: a qualitative and quantitative study. British Dental Journal 2005;198(9):571-8, discussion 59.

\section{Kobayashi 1964}

Kobayashi LY, Ash M. A clinical evaluation of an electric toothbrush used by orthodontic patients. Clinical Evaluation 1964;34(3):209-19.

\section{Kumar 2016}

Kumar S, Tadakamadla J, Johnson NW. Effect of toothbrushing frequency on incidence and increment of dental caries: a systematic review and meta-analysis. Journal of Dental Research 2016;95(11):1230-6.

\section{Kurbasic 2008}

Kurbasic I, Pandza H, Masic I, Huseinagic S, Tandir S, Alicajic F, et al. The advantages and limitations of international classification of diseases, injuries and causes of death from aspect of existing health care system of Bosnia and Herzegovina. Acta Informatica Medica 2008;16(3):159-61.

\section{Lamont 2018}

Lamont L, Worthington HV, Clarkson JE, Beirne PV. Routine scale and polish for periodontal health in adults. Cochrane Database of Systematic Reviews 2018, Issue 12. [DOI: 10.1002/14651858.CD004625.pub5]

\section{Lange 1977}

Lange DE, Plagmann HC, Eenboom A, Promesberger A. Clinical methods for the objective evaluation of oral hygiene [Klinische Methoden zur objektiven Beurteilung der Mundhygiene]. Deutsche Zahnarztliche Zeitschrift 1977;32(1):44-7.

\section{Lobene 1986}

Lobene RR, Weatherford T, Ross NM, Lamm RA, Menaker L. A modified gingival index for use in clinical trials. Clinical Preventive Dentistry 1986;8(1):3-6.

\section{Löe 1963}

Löe H, Silness J. Periodontal disease in pregnancy. I. Prevalence and severity. Acta Odontologica Scandinavica 1963;21:533-51.

\section{Löe 1965}

Löe H, Theilade E, Jensen SB. Experimental gingivitis in man. Journal of Periodontology 1965;36:177-87.

\section{Löe 1967}

Löe H. The Gingival Index, the Plaque Index and the Retention Index systems. Journal of Periodontology 1967;38(6 Part II):610-6.

\section{Löe 2000}

Löe $\mathrm{H}$. Oral hygiene in the prevention of caries and periodontal disease. International Dental Journal 2000;50(3):129-39.

\section{Mac Giolla Phadraig 2013}

Mac Giolla Phadraig C, Guerin S, Nunn J. Train the trainer? A randomized controlled trial of a multi-tiered oral health education programme in community-based residential services for adults with intellectual disability. Community Dentistry and Oral Epidemiology 2013;41(2):182-92.

\section{Michie 2011}

Michie S, Van Stralen MM, West R. The behaviour change wheel: a new method for characterising and designing behaviour change interventions. Implementation Science 2011;6(1):42.

\section{Michie 2013}

Michie S, Richardson M, Johnston M, Abraham C, Francis J, Hardeman W, et al. The behavior change technique taxonomy (v1) of 93 hierarchically clustered techniques: building an international consensus for the reporting of behavior change interventions. Annals of Behavioral Medicine 2013;46(1):81-95.

\section{Minihan 2014}

Minihan PM, Morgan JP, Park A, Yantsides KE, Nobles CJ, Finkelman MD, et al. At-home oral care for adults with developmental disabilities. Journal of the American Dental Association 2014;145(10):1018-25. 


\section{Moher 2001}

Moher D, Schulz KF, Altman DG. The CONSORT statement: revised recommendations for improving the quality of reports of parallel-group randomised trials. Lancet 2001;357(9263):1191-4.

\section{Mombelli 1998}

Mombelli AW. The role of dental plaque in the initiation and progression of periodontal diseases. European Workshop on Mechanical Plaque Control; 199805 09-12; Switzerland. Berlin: Quintessence Publishing Company Inc., 1998:85-97.

\section{Morgan 2012}

Morgan JP, Minihan PM, Stark PC, Finkelman MO, Yantsides KE, Park A, et al. The oral health status of 4,732 adults with intellectual and developmental disabilities. Journal of the American Dental Association 2012;143(8):838-46.

\section{Newton 2015}

Newton JT, Asimakopoulou K. Managing oral hygiene as a risk factor for periodontal disease: a systematic review of psychological approaches to behaviour change for improved plaque control in periodontal management. Journal of Clinical Periodontology 2015;42:S36-46.

\section{Oliveira 2013}

Oliveira JS, Prado Júnior RR, De Sousa Lima KR, De Oliveira Amaral H, Moita Neto JM, Mendes RF. Intellectual disability and impact on oral health: a paired study. Special Care in Dentistry 2013;33(6):262-8.

\section{Petersen 2013}

Petersen PE, Baez RJ. Oral Health Surveys: Basic Methods. 5th Edition. Geneva: World Health Organization, 2013.

\section{Prabhu 2010}

Prabhu NT, Nunn JH, Evans DJ, Girdler NM. Access to dental care - parents' and caregivers' views on dental treatment services for people with disabilities. Special Care in Dentistry 2010;30(2):35-45.

\section{Prangnell 2008}

Prangnell SJ, Green K. A cognitive behavioural intervention for dental anxiety for people with learning disabilities: a case study. British Journal of Learning Disabilities 2008;36(4):242-8.

\section{Rawlinson 2001}

Rawlinson SR. The dental and oral care needs of adults with a learning disability living in a rural community: consideration of the issues. Journal of Intellectual Disabilities 2001;5(2):133-56.

\section{Renz 2007}

Renz A, Ide M, Newton T, Robinson P, Smith D. Psychological interventions to improve adherence to oral hygiene instructions in adults with periodontal diseases. Cochrane Database of Systematic Reviews 2007, Issue 2. [DOI: 10.1002/14651858.CD005097.pub2]

\section{Review Manager 2014 [Computer program]}

The Nordic Cochrane Centre, The Cochrane Collaboration. Review Manager (RevMan). Version 5.3. Copenhagen: The Nordic Cochrane Centre, The Cochrane Collaboration, 2014.

\section{Riley 2013}

Riley P, Worthington HV, Clarkson JE, Beirne PV. Recall intervals for oral health in primary care patients. Cochrane Database of Systematic Reviews 2013, Issue 12. [DOI: 10.1002/14651858.CD004346.pub4]

\section{Sagheri 2013}

Sagheri D, McLoughlin J, Nunn JH. Dental caries experience and barriers to care in young children with disabilities in Ireland. Quintessence International 2013;44(2):159-69.

\section{Saxer 1975}

Saxer UP, Mühlemann HR. Motivation and education. Schweizerische Monatsschrift fur Zahnheilkunde 1975;85(9):9005-19.

\section{Schalock 2010}

Schalock RL, Borthwick-Duffy SA, Bradley VJ, Buntinx WH, Coulter DL, Craig EM, et al. Intellectual disability: definition, classification, and systems of supports. Washington, DC: American Association on Intellectual and Developmental Disabilities; 2010. Report No.: 978-1-9353-0404-3.

\section{Schou 1998}

Schou L. Behavioral aspects of dental plaque control measures: An oral health promotion perspective. European Workshop on Mechanical Plaque Control; 1998 May 9-12; Switzerland. Berlin: Quintessence Publishing Company Inc., 1998:287-99.

\section{Schünemann 2013}

Schünemann H, Brożek J, Guyatt G, Oxman A. Handbook for grading the quality of evidence and the strength of recommendations using the GRADE approach, 2013. gdt.guidelinedevelopment.org/app/handbook/ handbook.html\#h.52ccy41iwbon (accessed 26 March 2017).

\section{Shaw 1977}

Shaw L, Murray JJ. A new index for measuring extrinsic stain in clinical trials. Community Dental and Oral Epidemiology 1977;5:116-20.

\section{Silness 1964}

Silness J, Löe H. Periodontal disease in pregnancy II. Correlation between oral hygiene and periodontal condition. Acta Odontologica Scandinavica 1964;22(1):121-35.

\section{Soldani 2018}

Soldani FA, Lamont T, Jones K, Young L, Walsh T, Lala R, Clarkson JE. One-to-one oral hygiene advice provided in a dental setting for oral health. Cochrane Database of Systematic Reviews 2018, Issue 10. [DOI: 10.1002/14651858.CD007447.pub2]

\section{Sterne 2016}

Sterne JA, Hernán MA, Reeves BC, Savović J, Berkman ND, Viswanathan M, et al. ROBINS-I: a tool for assessing risk 
of bias in non-randomised studies of interventions. $B M J$ 2016;355:i4919.

\section{Stewart 1996}

Stewart J, Wolfe G, Maeder L, Hartz G. Changes in dental knowledge and self-efficacy scores following interventions to change oral hygiene behavior. Patient Education and Counselling 1996;26:269-77.

\section{Taylor-Dillon 2003}

Taylor-Dillon F, Friel S, Sixsmith J, Kelleher C. A review of oral health promotion/education activity in the Republic of Ireland and a study of attitudes, knowledge and behaviour of special needs groups towards oral health. Galway: Centre for Health Promotion Studies, National University of Ireland, Galway; 2003.

\section{Turesky 1970}

Turesky S, Gilmore ND, Glickman I. Reduced plaque formation by the chloromethyl analogue of victamine C. Journal of Periodontology 1970;41(1):41-3.

\section{Van der Weijden 2011}

Van der Weijden F, Slot DE. Oral hygiene in the prevention of periodontal diseases: the evidence. Periodontology 2000 2011;55(1):104-23.

\section{Waldron 2016}

Waldron C, Mac Giolla Phadraig C, Comiskey C, Nunn J. Results of a scoping review of oral health interventions for children or adolescents with disabilities. Journal of Disability and Oral Health 2016;17(4):144-9.

\section{Watt 2005}

Watt RG, Marinho VC. Does oral health promotion improve oral hygiene and gingival health?. Periodontology 2000 2005;37(1):35-47.

\section{Whelton 2009}

Whelton H, Crowley E, Nunn J, Murphy A, Kelleher V, Guiney H, et al. Oral Health of Children Attending Special Needs Schools and Day Care Centres. Dublin: University College Cork (UCC), Department of Health and Children (DOHC), 2009.

\section{WHO 1978}

WHO Scientific Group on Epidemiology Etiology and Prevention of Periodontal Diseases. Epidemiology, Etiology and Prevention of Periodontal Diseases: Report of a WHO Scientific Group. Geneva: World Health Organization, 1978.

\section{WHO 2001}

World Health Organization. The International Classification of Functioning, Disability and Health. Geneva: World Health Organization, 2001.

\section{WHO 2011}

World Health Organization. International Statistical Classification of Diseases and Related Health Problems. Geneva: World Health Organization, 2011.

\section{Willems 2017}

Willems Ml, Hilgenkamp TI, Havik E, Waninge AM, Craig A. Use of behaviour change techniques in lifestyle change interventions for people with intellectual disabilities: a systematic review. Research in Developmental Disabilities 2017;60:256-68.

\section{Williamson 2017}

Williamson PR, Altman DG, Bagley H, Barnes KL, Blazeby JM, Brookes ST, et al. The COMET handbook: version 1.0. Trials 2017;18(3):280.

\section{Worthington 2015}

Worthington $\mathrm{H}$, Clarkson J, Weldon J. Priority oral health research identification for clinical decision-making. Evidencebased Dentistry 2015;16(3):69-71.

\section{Yaacob 2014}

Yaacob M, Worthington HV, Deacon SA, Deery C, Walmsley DA, Robinson PG, et al. Powered versus manual toothbrushing for oral health. Cochrane Database of Systematic Reviews 2014, Issue 6. [DOI: 10.1002/14651858.CD002281.pub3]

\section{Young 2011}

Young T, Hopewell S. Methods for obtaining unpublished data. Cochrane Database of Systematic Reviews 2011, Issue 11. [DOI: 10.1002/14651858.MR000027.pub2]

\section{Zeller 2004}

Zeller A. Clinical Investigation of the Brushing Effectiveness and Manageability of an Electronic Toothbrush, a Three-Headed Manual Toothbrush and a Conventional Manual Toothbrush in Patients with Disabilities [Klinische Untersuchung der Putzeffektivität und Handhabbarkeit einer elekronischen Zahnbürste, einer dreiköpfigen Handzahnbürste und einer konventionellen Handzahnbürste bei Patienten mit Behinderungen]. [Masters Thesis]. Berlin: Freie Universität Berlin 2004.

\section{Zimmermann 2015}

Zimmermann H, Zimmermann N, Hagenfeld D, Veile A, Kim T$\mathrm{S}$, Becher $\mathrm{H}$. Is frequency of tooth brushing a risk factor for periodontitis? A systematic review and meta-analysis. Community Dentistry and Oral Epidemiology 2015;43(2):116-27.

\section{References to other published versions of this review}

\section{Waldron 2017}

Waldron C, MacGiolla Phadraig C, Nunn J, Comiskey C, Donnelly-Swift E, Guerin S, et al. Oral hygiene programmes for people with intellectual disabilities. Cochrane Database of Systematic Reviews 2017, Issue 4. [DOI: 10.1002/14651858.CD012628]

* Indicates the major publication for the study 
CHARACTERISTICS OF STUDIES

Characteristics of included studies [ordered by study ID]

Abramson 1972

\begin{tabular}{|c|c|}
\hline \multirow[t]{7}{*}{ Methods } & Study design: repeat measure \\
\hline & Date of study: not given, pre-1972 \\
\hline & Study duration: unclear \\
\hline & Location: USA \\
\hline & Setting: residential \\
\hline & Ethical approval: none reported \\
\hline & Consent: none reported \\
\hline \multirow[t]{8}{*}{ Participants } & $\begin{array}{l}\text { Description of ID used: "severely retarded;" "The mean MA (Mental Age) as measured by the Peabody } \\
\text { Picture Vocabulary Test was } 2 \text { years and } 8 \text { months. The mental ages ranged from } 2 \text { years } 2 \text { months to } 4 \\
\text { years } 1 \text { months." }\end{array}$ \\
\hline & Conversion to ICD description: severe \\
\hline & Age range (mean): 9 years 3 months to 14 years 8 months ( 12 years 4 months) \\
\hline & Sex: male \\
\hline & Comorbidity reported/details: none reported \\
\hline & Number of participants at baseline: 9 \\
\hline & Number of participants at final evaluation: 8 \\
\hline & Selection of the participants for the intervention: no details \\
\hline
\end{tabular}

Interventions

\section{Comparison: training of people with ID versus no training}

Intervention: teach dental hygiene techniques to people with ID using discrimination, reinforcement and modelling training. Brushing behaviour based on a checklist of 19 items

Outcomes

1. Sessions required to reach discrimination

2. Steps achieved in a 19-step toothbrushing routine using modelling and reinforcement with rewards

Timing of outcome assessments: 8 observations at baseline and 8 post-training

$$
\text { COM-B System Character- Potential sources of behaviour change: capability (physical), motivation (reflective) }
$$

istics

Potential Intervention functions: training, modelling, incentivisation

Stakeholder Involvement Formal or non-formal carer: formal

With or without dental professional involvement: without

Other stakeholders: 1 counsellor (staff member) trained to use the checklist of 19 steps to brushing

Strengths and weaknesses: duration of the intervention unclear
Modifications to the intervention: none reported
Adverse effects: none reported


Funding source: "Supported by Grant RD - 2286-P068Cl of the Social and Rehabilitation Services, Dept of Health, Education and Welfare." Comment: unlikely to be a conflict.

\section{Risk of bias}

\begin{tabular}{|c|c|c|}
\hline Bias & Authors' judgement & Support for judgement \\
\hline $\begin{array}{l}\text { Was the intervention inde- } \\
\text { pendent of other changes? } \\
\text { (ITS) }\end{array}$ & Unclear risk & $\begin{array}{l}\text { Comment: none stated; other changes or events may have influenced the out- } \\
\text { comes. Study duration unclear }\end{array}$ \\
\hline $\begin{array}{l}\text { Was the shape of the in- } \\
\text { tervention effect prespeci- } \\
\text { fied? (ITS) }\end{array}$ & Low risk & Comment: the point of analysis was the point of intervention. \\
\hline $\begin{array}{l}\text { Was the intervention un- } \\
\text { likely to affect data collec- } \\
\text { tion? (ITS) }\end{array}$ & Low risk & $\begin{array}{l}\text { Comment: the intervention did not affect either the source or method of data } \\
\text { collection. }\end{array}$ \\
\hline $\begin{array}{l}\text { Was knowledge of the al- } \\
\text { located interventions ade- } \\
\text { quately prevented during } \\
\text { the study? (ITS) }\end{array}$ & High risk & $\begin{array}{l}\text { Comment: not possible to blind. All participants in the intervention known to } \\
\text { rater and researcher. }\end{array}$ \\
\hline $\begin{array}{l}\text { Were incomplete out- } \\
\text { come data adequately ad- } \\
\text { dressed? (ITS) }\end{array}$ & Low risk & $\begin{array}{l}\text { Comment: } 1 \text { participant did not complete the training as they moved from the } \\
\text { setting. Unlikely to affect outcomes. }\end{array}$ \\
\hline $\begin{array}{l}\text { Was the study free from } \\
\text { selective outcome report- } \\
\text { ing? (ITS) }\end{array}$ & Low risk & Comment: all outcome data were reported. \\
\hline
\end{tabular}

Albino 1979

Study design: RCT
Date of study: not given, pre-winter 1978
Study duration: 5 weeks
Location: USA
Setting: school
Ethical approval: not reported
Consent: not reported
Description of ID used: "severely retarded children;" "IQ between 20 and 40"
Conversion to ICD description: moderate and severe
Age range: $3-21$ years
Sex: both
Comorbidity reported/details: yes "Down syndrome, Tay-Sachs disorder, epilepsy, muscular dystrophy,
Turner syndrome, autism, hydrocephaly, Hunter syndrome, Prader-Willi syndrome and PKU disorder."
Schwartz 1978, p. 18


Albino 1979 (Continued)

Number of participants at baseline: 31

Number of participants at final evaluation: 36 . Comment: 11 of the post-test sample were not in the pretest sample. In total, pre and post data were available for 26 participants: 17 in intervention group, 9 in control group.

Selection of the participants for the intervention: 58 children attending a summer school. Only those allowing the oral examination were reported.

\section{Interventions Comparison: training of people with ID versus no training}

Intervention: orientation and desensitisation (11 sessions), social (verbal and physical praise) and tangible (stars traded for small toys) reinforcement. Individualisation and parental involvement; individual feedback on their children's progress along with suggestions that they might use at home to enhance and reinforce learning. Schwartz 1978, p. 20.

Control: orientation and desensitisation (11 sessions) only

1. Plaque Index - Kobayashi \& Ash (6 teeth scored) (V). This index is a modification of Ramjford and
Schick \& Ash
Timing of outcome assessments: baseline and final

COM-B System Character- Potential sources of behaviour change: capability (physical/psychological), opportunity (social/physiistics cal), motivation (reflective)

Potential intervention functions: training, enablement, incentivisation, environmental restructuring

$\begin{array}{ll}\text { Stakeholder Involvement } & \text { Formal or non-formal carer: both } \\ & \begin{array}{l}\text { With or without dental professional involvement: with - dental instructors, dental student, predental } \\ \text { student }\end{array} \\ & \text { Other stakeholder involvement: parents, director of centre }\end{array}$

Strengths and weakness: high attrition rate
Modifications to the intervention: the intervention was continuously modified to meet the needs of
individual participants. Quote: "it soon became obvious that all children were not able to master the
[toothbrushing] technique and attention was given to individualisation" Schwartz 1978, p. 20.
Adverse effects: none reported
Funding source: quote: "Funding for this study was provided in part by a grant from Johnson and John-
son, Inc." Albino 1979, p. 28. Comment: unlikely to be a conflict.

\section{Risk of bias}

\begin{tabular}{lll}
\hline Bias & Authors' judgement & Support for judgement \\
\hline $\begin{array}{l}\text { Random sequence genera- } \\
\text { tion (selection bias) }\end{array}$ & Low risk & $\begin{array}{l}\text { Quote: "Half the children were randomly assigned to a control group and the } \\
\text { other half to an experimental group." (Schwartz 1978, p. 18). }\end{array}$ \\
\hline $\begin{array}{l}\text { Allocation concealment } \\
\text { (selection bias) }\end{array}$ & Unclear risk & Comment: no details reported \\
\hline $\begin{array}{l}\text { Blinding of participants } \\
\begin{array}{l}\text { and personnel (perfor- } \\
\text { mance bias) }\end{array}\end{array}$ & Unclear risk & $\begin{array}{l}\text { Comment: no details provided but control group did receive some interven- } \\
\text { All outcomes }\end{array}$ \\
\hline
\end{tabular}


Albino 1979 (Continued)

Blinding of outcome as- High risk Comment: examiner was not blinded. sessment (detection bias)

All outcomes

Quote: "The examiner was also the student dentist acting as instructor in the program." (Albino 1979, p. 27).

Incomplete outcome data High risk

Comment: level of attrition likely to impact on outcome effect.

(attrition bias)

Quote: "attrition rate of subjects particularly when it was occasioned by fear

All outcomes and lack of appropriate behaviors, was discouraging." (Albino 1979, p. 27).

Selective reporting (re- High risk porting bias)

Comment: criteria for scoring plaque was modified due to difficulties in recording all surfaces. Reduced from 12 to 8 surfaces, no SD given.

Altabet 2003

Study design: NRCT "2 2 repeated measures factorial design with two groups (tx [treatment], no tx)
both measured over two distinct time periods," p. 144.
Date of study: 20 March 1999 to 20 March 2000
Study duration: 12 months
Location: USA
Setting: residential
Ethical approval: not reported
Consent: not reported

Participants

Description of ID used: mild to moderate and severe to profound "range of mental retardation"

Conversion to ICD description: mixed

Age range: $22-57$ years

Sex: male 44, female 35

Comorbidity reported/details: none

Number of participants at baseline: 79

Number of participants at final evaluation: 79

Selection of the participants for the intervention: residents who attended for a dental examination on 3 occasions within the year of the study.

\begin{tabular}{ll}
\hline Interventions & Comparison: individualised oral care plan versus usual care \\
& Group 1: individualised oral care plan (appendix A, p. 444) \\
& $\begin{array}{l}\text { Group 2: continued regular oral hygiene care, which was supported by a carer following guidelines (ap- } \\
\text { pendix B, p. 445) }\end{array}$ \\
\hline Outcomes & $\begin{array}{l}\text { 1. Rating of oral hygiene } 1 \text { to } 5 \text { (excellent to very poor), averaged over the designated time periods, p. } \\
\text { Timing of outcome assessments: baseline, midway and final }\end{array}$ \\
\hline $\begin{array}{l}\text { COM-B System Character- } \\
\text { istics }\end{array}$ & $\begin{array}{l}\text { Potential sources of behaviour change: capability (physical /psychological), opportunity (social/physi- } \\
\text { cal), motivation (reflective). }\end{array}$ \\
\hline
\end{tabular}


Altabet 2003 (Continued)

Potential intervention functions: training, modelling, enablement, persuasion, environmental restructuring

\begin{tabular}{ll}
\hline Stakeholder Involvement $\quad$ & Formal or non-formal carer: formal \\
& With or without dental professional involvement: with - dentist and dental hygienists \\
& Other stakeholder involvement: used to determine the individualised care plan - psychology or behav- \\
& iour analysis, dental department referral, occupational therapist, speech-language pathologist, nurs- \\
& ing or medical referral, physical therapy, case management, procurement of special equipment, pa- \\
& tient education, unit supervisors, direct support staff. Direct care staff provided the day-to-day oral care \\
(appendix A, p. 444)
\end{tabular}

Strengths and weaknesses: non
Modifications to the interventio
tervention.
Adverse effects: none reported
Funding source: none reported

\section{Risk of bias}

\begin{tabular}{lll}
\hline Bias & Authors' judgement & Support for judgement \\
\hline $\begin{array}{l}\text { Random sequence genera- } \\
\text { tion (selection bias) }\end{array}$ & High risk & $\begin{array}{l}\text { Quote: "based on timeliness of their referral, with earlier referrals receiving the } \\
\text { individualized oral care plans and the remaining individuals placed on a wait- } \\
\text { ing list," p. 441. }\end{array}$ \\
\hline $\begin{array}{l}\text { Allocation concealment } \\
\text { (selection bias) }\end{array}$ & High risk & $\begin{array}{l}\text { Comment: those attending early in the year were in the intervention group, p. } \\
441 .\end{array}$ \\
\hline $\begin{array}{l}\text { Blinding of participants } \\
\text { and personnel (perfor- } \\
\text { mance bias) } \\
\text { All outcomes }\end{array}$ & Low risk & Comment: most likely low as study took place at 2 different time points \\
\hline $\begin{array}{l}\text { Blinding of outcome as- } \\
\text { sessment (detection bias) } \\
\text { All outcomes }\end{array}$ & Low risk & $\begin{array}{l}\text { Quote: "The raters (facility dentist and dental hygienist) were not aware of who } \\
\text { had participated in each group," p. 441. }\end{array}$ \\
\hline $\begin{array}{l}\text { Incomplete outcome data } \\
\begin{array}{l}\text { (attrition bias) } \\
\text { All outcomes }\end{array}\end{array}$ & Low risk & Comment: no missing outcome data \\
\hline $\begin{array}{l}\text { Selective reporting (re- } \\
\text { porting bias) }\end{array}$ & Unclear risk & Comment: no protocol, but all listed outcomes reported \\
\hline
\end{tabular}

Bickley 1990

$\begin{array}{ll}\text { Methods } & \text { Study design: RCT } \\ & \text { Date of study: not reported, pre-1990 } \\ & \text { Study duration: } 6 \text { months } \\ & \text { Location: UK }\end{array}$


Bickley 1990 (Continued)

Setting: day centre

Ethical approval: not reported

Consent: quote: "Clients agreement to participate was confirmed," p. 4.

Description of ID used: "mentally handicapped"
Conversion to ICD description: unclear
Age range: not reported
Sex: not reported
Comorbidity reported/details: none reported
Number of participants at baseline: 30
Number of participants at final evaluation: 29
Selection of the participants for the intervention: following a routine dental examination, identified as
having poor oral hygiene

Interventions

Comparison: discussion of individual clinical photographs used as motivators versus no discussion for people with ID

Group 1: monthly photographic records of individuals, with their plaque disclosed, used to compare and discuss their oral hygiene from visit to visit, with related $\mathrm{OHI}$

Group 2: monthly photographic records of individuals, with their plaque disclosed, but not used to compare; general $\mathrm{OHI}$
Outcomes
1. Plaque, calculus, pocketing, bleeding indices of 6 teeth based on WHO diagnostic criteria (V)
2. Extrinsic Stain Index (Shaw \& Murray 1977) (V)
3. Cognitive and perceptual learning (interviews)
Timing of outcome assessments: 1 . baseline and final, 2. monthly
COM-B System Character- Potential sources of behaviour change: capability (physical/psychological), motivation (reflective) istics

Potential intervention functions: enablement, persuasion, environmental restructuring

\section{Stakeholder Involvement Formal or non-formal carer: N/A}

With or without dental professional involvement: with - dental hygienist

Other stakeholder involvement: none

Strengths and weaknesses: lack of direct contact between investigators and carers was a disadvantage
Modifications to the intervention: none
Adverse effects: none reported
Funding source: quote: "For the provision of toothbrushes and toothpaste, we gratefully acknowledge
the following companies Oral B, Colgate and Gibbs Mentadent." Comment: no likely conflict

\section{Risk of bias}


Bickley 1990 (Continued)

Random sequence genera- Low risk Quote: "after the baseline examination the subjects were allocated using a tion (selection bias) random numbers table into the test or the control group," p. 4.

\begin{tabular}{lll}
\hline $\begin{array}{l}\text { Allocation concealment } \\
\text { (selection bias) }\end{array}$ & Unclear risk & Comment: no details provided \\
\hline $\begin{array}{l}\text { Blinding of participants } \\
\text { and personnel (perfor- }\end{array}$ & Unclear risk & Comment: no details provided \\
mance bias) & & \\
All outcomes & &
\end{tabular}

\begin{tabular}{lll}
\hline $\begin{array}{l}\text { Blinding of outcome as- } \\
\text { sessment (detection bias) } \\
\text { All outcomes }\end{array}$ & Low risk & Comment: assessor was not involved in the study. \\
\hline $\begin{array}{l}\text { Incomplete outcome data } \\
\text { (attrition bias) } \\
\text { All outcomes }\end{array}$ & Low risk & $\begin{array}{l}\text { Comment: unclear why } 1 \text { participant dropped out, but unlikely to affect out- } \\
\text { come }\end{array}$ \\
\hline $\begin{array}{l}\text { Selective reporting (re- } \\
\text { porting bias) }\end{array}$ & High risk & $\begin{array}{l}\text { Comment: only the plaque index was reported from the full assessments. Re- } \\
\text { sults of cognitive and perceptual interviews not reported. Poor reporting of } \\
\text { measures used. Unable to contact author for more details. }\end{array}$ \\
\hline
\end{tabular}

\section{Bildt 2010}

Methods

Study design: RCT

Date of study: not given, pre-August 2010

Study duration: 4 months

Location: the Netherlands

Setting: unclear

Ethical approval: yes, "approved by the Medical Ethics Review Committee of the University Medical Center Groningen (Registration number: NL 28061.042.09)"

Consent: unclear. Carers were asked for "permission." No report of consent from participants

Participants

Description of ID used: IQ < 50, "entirely dependent on another for their oral care"

Conversion to ICD description: moderate to profound

Age range: not provided. Mean age: male 28 years, female 31 years

Sex: males 12 , females 6

Comorbidity reported/details: physical disabilities

Number of participants at baseline: 18

Number of participants at final evaluation: 18

Selection of the participants for the intervention: from 2 centres for Special Care Dentistry

Interventions

\section{Comparison: special 3-headed manual toothbrush used by carer versus conventional manual toothbrush used by carer}

Group 1: Superbrush used by carer on person with ID following $\mathrm{OHI}$ 
Bildt 2010 (Continued)

Group 2: Oral B Indicator used by carer on person with ID following $\mathrm{OHI}$

1. Green \& Vermillion Plaque Index (scale 0 - 18 - mean of 6 standard teeth)
2. Ainamo \& Bay Gingival Bleeding Index (scale $0-1,6$ teeth, \% of positive sites)
3. Questionnaire for carers, 5 questions (frequency of brushing, experience using the brush, handling of
toothbrush by carer, most difficult sextant to brush for carer and level of resistance of people with ID)
Timing of outcome assessments: baseline and 4 months

COM-B System Character- Potential sources of behaviour change: capability (physical), opportunity (physical)

istics

Potential intervention functions: training, enablement, environmental restructuring

Stakeholder Involvement Formal or non-formal carer: both

With or without dental professional involvement: with - dentist

Other stakeholder involvement: no

Notes
Modifications to the intervention: no
Adverse effects: assessed in carers' questionnaire - small number of participants reacted to the size of
the Superbrush when first used
Funding source: none reported

\section{Risk of bias}

\begin{tabular}{|c|c|c|}
\hline Bias & Authors' judgement & Support for judgement \\
\hline $\begin{array}{l}\text { Random sequence genera- } \\
\text { tion (selection bias) }\end{array}$ & Low risk & Quote: "closed envelopes" \\
\hline $\begin{array}{l}\text { Allocation concealment } \\
\text { (selection bias) }\end{array}$ & Low risk & Comment: use of closed envelopes probably ensured allocation concealment. \\
\hline $\begin{array}{l}\text { Blinding of participants } \\
\text { and personnel (perfor- } \\
\text { mance bias) } \\
\text { All outcomes }\end{array}$ & Low risk & $\begin{array}{l}\text { Comment: some attempt to blind carers as new toothbrushes and } \mathrm{OHI} \text { were } \\
\text { given to control group. Blinding or not of the participants may not have im- } \\
\text { pacted on the outcome as teeth were brushed by the carers. }\end{array}$ \\
\hline $\begin{array}{l}\text { Blinding of outcome as- } \\
\text { sessment (detection bias) } \\
\text { All outcomes }\end{array}$ & Low risk & Comment: assessors were blinded to the group allocations. \\
\hline $\begin{array}{l}\text { Incomplete outcome data } \\
\text { (attrition bias) } \\
\text { All outcomes }\end{array}$ & Low risk & Comments: no missing data \\
\hline $\begin{array}{l}\text { Selective reporting (re- } \\
\text { porting bias) }\end{array}$ & Unclear risk & Comment: no protocol, but all outcomes in Methods section reported. \\
\hline
\end{tabular}

Bouter 1979

Methods Study design: repeat measure


Date of study: not reported, pre-1979

Study duration: unclear - varied from participant to participant - maximum 100 days

Location: the Netherlands

Setting: residential

Ethical approval: not reported

Consent: not reported

Pescription of ID used: "IQ varied from <35 to 46"
Conversion to ICD description: moderate
Age range (mean): $20-28$ years (25 years)
Sex: male 4, female 4
Comorbidity reported/details: none reported
Number of participants at baseline: 8
Number of participants at final evaluation: 7
Selection of the participants for the intervention: "ability to imitate non verbal behaviours and to un-
derstand verbal instruction," p. 63.

Interventions

\section{Comparison: training of people with ID versus no training}

Group 1: acquisition of skills and duration training in 15 steps of a toothbrushing routine, with 5 levels of assistance and 4 levels of feedback/praise during the acquisition stage.

Group 2: no training

Duration training $=$ all steps completed without assistance or feedback

Outcomes $\begin{aligned} & \text { 1. Acquisition of } 15 \text { steps in toothbrushing routine and the number of sessions required to achieve } \\ & \text { these steps. Trainer recorded type of assistance technique required and type of feedback used }\end{aligned}$
2. Measure of level of maintenance of the skill over $\geq 1$ week
Timing of outcome assessments: varied between participants - baseline to final - $\geq 5$ measures per
week reported for all. Follow-up/duration stage was measured at increasing intervals, progressed from
2-, 3-, 4-, 6-, 8-day intervals.

COM-B System Character- Potential sources of behaviour change: capability (physical /psychological), opportunity (physical), moistics tivation (reflective)

Potential Intervention functions: training, modelling, persuasion, environmental restructuring

\section{Stakeholder Involvement Formal or non-formal carer: formal}

With or without dental professional involvement: without

Other stakeholder involvement: none

Notes Strengths and weaknesses: time involved in training; "It is not expected that most care workers will have even that much time available (10 - 20 minutes) for each individual subject," p. 69.

Modifications to the intervention: yes, for 3 participants, steps required to progress to next step were reduced to 14 and feedback was not reduced until each step was completed on 3 consecutive occasions. 
Bouter 1979 (Continued)

Adverse effects: unclear - 1 participant felt ill. Researchers considered it a possible delaying tactic. Participant given incentive of an orange, p. 66 .

Funding source: none reported

\section{Risk of bias}

Bias Authors' judgement Support for judgement

Was the intervention independent of other changes? (ITS)
Unclear risk

Comment: none stated; other changes or events may have influenced the outcomes.

\begin{tabular}{lll}
\hline $\begin{array}{l}\text { Was the shape of the in- } \\
\text { tervention effect prespeci- } \\
\text { fied? (ITS) }\end{array}$ & Low risk \\
\hline $\begin{array}{l}\text { Was the intervention un- } \\
\text { likely to affect data collec- }\end{array}$ & Low risk & $\begin{array}{l}\text { Comment: the intervention did not affect either the source or method of data } \\
\text { collection }\end{array}$
\end{tabular}
tion? (ITS)

Was knowledge of the al- High risk $\quad$ Comment: not possible to blind
located interventions ade-
quately prevented during
the study? (ITS)

\begin{tabular}{|c|c|c|}
\hline $\begin{array}{l}\text { Were incomplete out- } \\
\text { come data adequately ad- } \\
\text { dressed? (ITS) }\end{array}$ & Low risk & $\begin{array}{l}\text { Comment: participant who did not complete the intervention was fully docu- } \\
\text { mented. }\end{array}$ \\
\hline $\begin{array}{l}\text { Was the study free from } \\
\text { selective outcome report- } \\
\text { ing? (ITS) }\end{array}$ & Unclear risk & $\begin{array}{l}\text { Comment: the trainer scored the participants, but appeared to be completely } \\
\text { impartial. }\end{array}$ \\
\hline
\end{tabular}

Bratel 1991

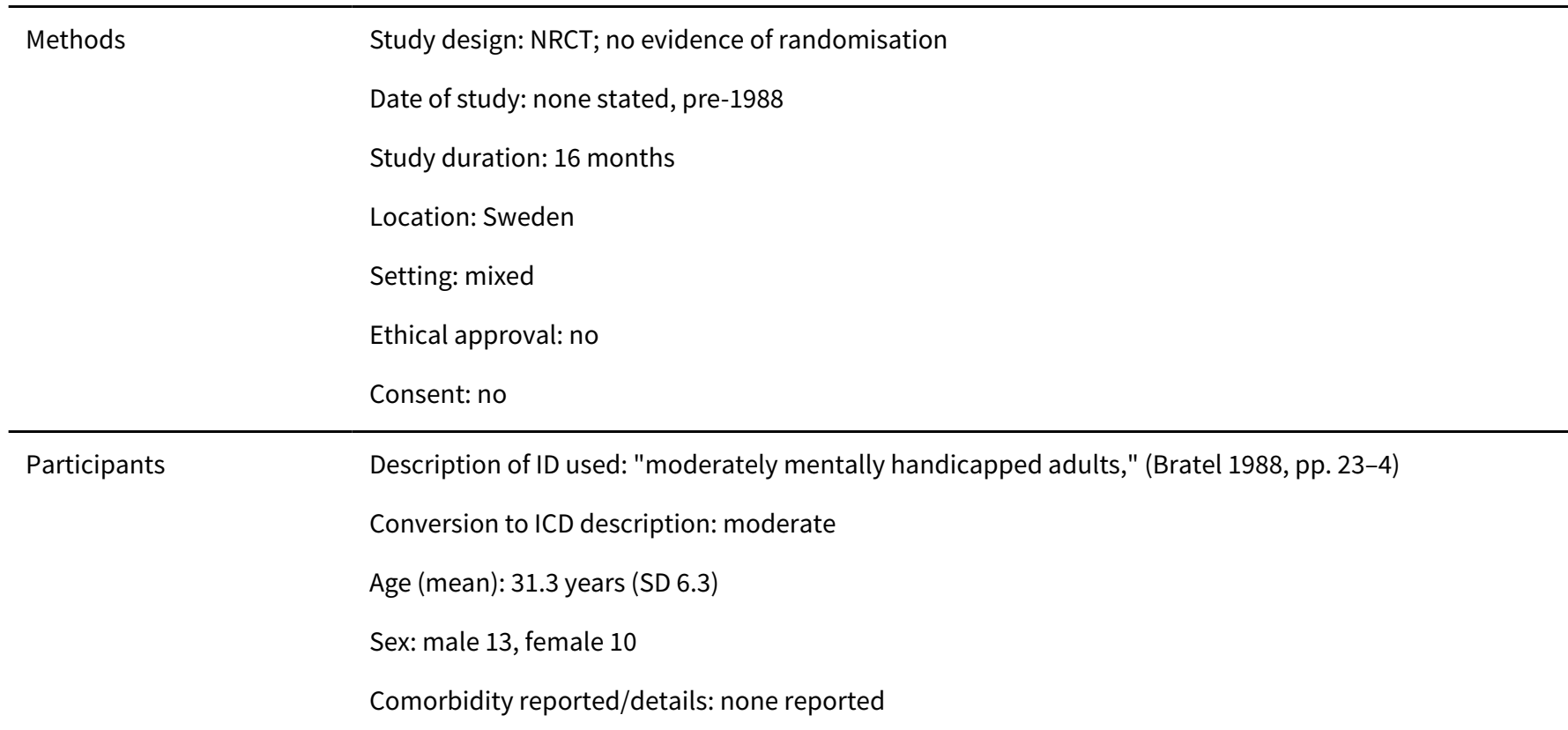


Bratel 1991 (Continued)

Number of participants at baseline: 23

Number of participants at final evaluation: 23 (and 22 for 1 outcome) (1 lost to follow-up in Group 3 see Table 3 in Bratel 1991, p. 6)

Selection of the participants for the intervention: from the dental hospital recall system (Bratel 1988, p. 26)

\section{Interventions Comparison: electric toothbrush versus manual toothbrush}

Standard oral health information provided to all: $\mathrm{OHI}$ according to Bass technique provide to participants and demonstrated in his/her own mouth (adapted to type of brush). Instructed to brush at least morning and night

Group 1: E1 - electric toothbrush (Braun Dental d3) unaided, i.e. no help when brushing

Group 2: E2 - electric toothbrush (Braun Dental d3) aided, i.e. with help from parents or staff. OHI given to parents and staff and demonstrated in participants' own mouths and practiced by them under supervision

Group 3: C1 - manual toothbrush unaided

Group 4: C2 - manual toothbrush aided, as in E2

We combined aided and unaided data

1. Silness \& Löe Plaque Index (V)

2. Silness \& Löe Gingival Index (V)

3. Supervision of wear and tear on toothbrushes

4. Diary of participant brushing kept by parents/staff

5. Questionnaire at baseline to assess current oral hygiene routines

6. Interview with those using electric toothbrush to assess their experiences using it

Timing of outcome assessment: baseline, 1 week, 4 weeks, 12 weeks, 16 months

COM-B System Characteristics
Potential sources of behaviour change: capability (physical/psychological), opportunity (social/physical)

Potential intervention functions: education, training, modelling, enablement, environmental restructuring

\section{Stakeholder Involvement Formal or non-formal carer: both}

With or without dental professional involvement: with - dentist, dental hygienist

Other stakeholder involvement: parents and staff carers involved in aiding groups E2 and C2

Adverse effects: yes, all reported in relation to electric toothbrush; "Patients reported some discomfort during brushing," "difficult to control the force and position of the electric toothbrush," Bratel 1988, p. 26; "toothbrush out of order (2 weeks);" "Patients reported some discomfort during brushing at the 3 month follow-up," (Bratel 1991, p. 6).

Funding source: none reported

\section{Risk of bias}


Bratel 1991 (Continued)

\section{Bias Authors' judgement Support for judgement}

Random sequence genera- Unclear risk tion (selection bias)

Quote: "a selected group of patients from the recall system." "Patients were divided into two examination groups and two control groups," (Bratel 1988, p. 24). Comment: no mention of randomisation.

\begin{tabular}{|c|c|c|}
\hline $\begin{array}{l}\text { Allocation concealment } \\
\text { (selection bias) }\end{array}$ & High risk & $\begin{array}{l}\text { Comment: not possible; those with more severe ID were aided (E2 and C2) } \\
\text { (Bratel 1988, p. 24). }\end{array}$ \\
\hline $\begin{array}{l}\text { Blinding of participants } \\
\text { and personnel (perfor- } \\
\text { mance bias) } \\
\text { All outcomes }\end{array}$ & Unclear risk & $\begin{array}{l}\text { Comment: not reported but different settings; most likely unaware of what } \\
\text { other groups were doing. }\end{array}$ \\
\hline $\begin{array}{l}\text { Blinding of outcome as- } \\
\text { sessment (detection bias) } \\
\text { All outcomes }\end{array}$ & Unclear risk & $\begin{array}{l}\text { Comment: all clinical measures were recorded by the lead author; no mention } \\
\text { of blinding. }\end{array}$ \\
\hline
\end{tabular}

Incomplete outcome data Low risk Comment: no missing data - diary entries were summarised.

(attrition bias)

All outcomes

Selective reporting (re- Unclear risk $\quad$ Comment: no protocol but all listed outcomes reported.
porting bias)
porting bias)

\section{Carr 1997}

Methods

Study design: cluster RCT - the group homes were the unit of randomisation, p. 134.

Date of study: not stated, pre-1997

Study duration: 12 months

Location: USA

Setting: residential

Ethical approval: no

Consent: yes, "Written consent was obtained," p. 133.

Participants Description of ID used: "Mental Retardation/Developmental Disability," p. 134

Conversion to ICD description: unclear

Age range (mean): 25-64 years (36.8 years)

Sex: male 32, female 24

Comorbidity reported/details: yes, cerebral palsy 5, hearing impaired 1, visually impaired 2

Number of participants at baseline: not given

Number of participants at final evaluation: "56 residents...completed the study"

Selection of the participants for the intervention: no details given 
Carr 1997 (Continued)

All groups - verbal OHI provided, then 1-to-1 instruction using the designated brush and practice on a typodont during initial instruction. Those requiring assistance were determined by testing their capability. p. 134

Group 1: self-brushing with Interplak electric toothbrush

Group 2: self-brushing with manual toothbrush (Oral B 40)

Group 3: assisted brushing with Interplak electric toothbrush

Group 4: assisted brushing with manual toothbrush (Oral B 40)

We combined data for self-brushers and assisted brushers for each toothbrush type.

Outcomes $\quad$ Outcomes measured on selected teeth "Buccal of \#3, \#4 and \#14. Lingual of \#19, Facial of \#30," p. 135

1. Gingival Index (Löe) (V)

2. Simplified Oral Hygiene Index (Green and Vermillion) (V)

3. Calculus measurement, p. 135

Timing of outcome assessments: baseline, 3 months, 6 months, 9 months and 12 months

COM-B System Character- Potential sources of behaviour change: capability (physical), opportunity (physical) istics

Potential intervention functions: training, modelling, environmental restructuring

\section{Stakeholder Involvement Formal or non-formal carer: formal}

With or without dental professional involvement: with - dental hygienist

Other stakeholder involvement: carers in group homes for those requiring assistance throughout study

Notes Strengths and weaknesses: quote: "The validity and reliability of the oral hygiene techniques used in the study must be regarded as limited. The examiners could not be present at all toothbrushing sessions and relied on staff for the implementation of tooth brushing protocols," p. 136.

Modifications to the intervention: none reported

Adverse effects: unclear - quote: "some subjects dismissed from study," p. 136.

Funding source: none reported

\section{Risk of bias}

Bias Authors' judgement Support for judgement

Random sequence genera- Unclear risk tion (selection bias)
Quote: "Each group home was randomly divided into two study groups, one using the Interplak and the second using a manual TB," p. 134.

Comment: no further details provided

\begin{tabular}{lll}
\hline $\begin{array}{l}\text { Allocation concealment } \\
\text { (selection bias) }\end{array}$ & Unclear risk & Comment: no details provided \\
\hline $\begin{array}{l}\text { Blinding of participants } \\
\text { and personnel (perfor- } \\
\text { mance bias) } \\
\text { All outcomes }\end{array}$ & Unclear risk & $\begin{array}{l}\text { Comment: unclear if personnel were blinded; study was a cluster study so par- } \\
\text { ticipants were most likely blinded. }\end{array}$ \\
\hline $\begin{array}{l}\text { Blinding of outcome as- } \\
\text { sessment (detection bias) }\end{array}$ & Unclear risk & $\begin{array}{l}\text { Quote: "The data were obtained by a single examiner (SB), a dental hygienist } \\
\text { who did not know to which test group the residents had been assigned," but }\end{array}$ \\
\hline
\end{tabular}


Carr 1997 (Continued)

All outcomes

"Comparisons were made at the time of examination, along with specific recommendations for improvement of oral hygiene," p. 135.

\section{Incomplete outcome data Unclear risk}

(attrition bias)

All outcomes
Quote: "Some initial subjects had to be dismissed from the study because they either lost their Interplak for a time, it malfunctioned and /or they had to un-

dergo hospitalization, thus interrupting the oral hygiene protocol," p. 136.

Comment: no details of how many were dismissed.

Selective reporting (re- $\quad$ Unclear risk $\quad$ Comment: no protocol but all listed outcomes reported.
porting bias)

porting bias)

\section{Christen 2007}

Study design: RCT, 3-way cross-over trial
Date of study: not stated, prior to or during 2007
Study duration: 2 weeks for each arm, with 2-week washouts, 12 weeks in total
Location: Germany
Setting: residential and home
Ethical approval: yes
Consent: yes

Participants

Description of ID used: "varying degrees of intellectual and/or physical impairments" - author confirmed by email that all participants had ID.

Conversion to ICD description: mixed

Age range: $18-45$ years

Sex: 22 male, 14 female

Comorbidity reported/details: "varying degrees of intellectual and/or physical impairments"

Number of participants at baseline: 36

Number of participants at final evaluation: 36

Selection of the participants for the intervention: all attended a university clinic for people with special needs.
Interventions
Comparison: electric toothbrush versus manual toothbrush
Comparison: special manual toothbrush versus conventional manual toothbrush
Group 1: Dentacare-Sonodent electric toothbrush, with verbal and written instruction
Group 2: Superbrush special manual toothbrush with verbal and written instruction
Group 3: Oral B Cross manual toothbrush with verbal and written instruction
The same toothpaste was used by all groups

Outcomes $\begin{aligned} & \text { 1. Modified Quigley-Hein Plaque Index (V) } \\ & \text { 2. API (V) }\end{aligned}$


Christen 2007 (Continued)

\section{Papilla Bleeding Index (V)}

Timing of outcome assessments: baseline and week 2 for each of the 3 cross-over time points, with 2week washout periods

\section{COM-B System Character- Potential sources of behaviour change: capability (physical), opportunity (physical)}

istics

Potential Intervention functions: training, enablement, environmental restructuring

\begin{tabular}{ll} 
Stakeholder Involvement & $\begin{array}{l}\text { Formal or non-formal carer: both } \\
\text { With or without dental professional involvement: with - dentist } \\
\text { Other stakeholder involvement: none reported }\end{array}$ \\
\hline Notes & Strengths and weaknesses: short duration \\
& Modifications to the intervention: none reported \\
& Adverse effects: yes, noise and vibration of electric toothbrush, size of head of both electric and Super- \\
brush & \\
Funding source: none reported
\end{tabular}

\section{Risk of bias}

\begin{tabular}{|c|c|c|}
\hline Bias & Authors' judgement & Support for judgement \\
\hline $\begin{array}{l}\text { Random sequence genera- } \\
\text { tion (selection bias) }\end{array}$ & Low risk & $\begin{array}{l}\text { Quote: "In order to ensure the reliability, the classification into order groups } \\
\text { and the allocation of the test toothbrushes was carried out by means of a lot- } \\
\text { tery procedure by a third person." }\end{array}$ \\
\hline $\begin{array}{l}\text { Allocation concealment } \\
\text { (selection bias) }\end{array}$ & Low risk & $\begin{array}{l}\text { Quote: "The allocation of the test toothbrushes was carried out by means of a } \\
\text { lottery procedure by a third person." }\end{array}$ \\
\hline $\begin{array}{l}\text { Blinding of participants } \\
\text { and personnel (perfor- } \\
\text { mance bias) } \\
\text { All outcomes }\end{array}$ & High risk & Comment: not possible to blind participants or personnel \\
\hline $\begin{array}{l}\text { Blinding of outcome as- } \\
\text { sessment (detection bias) } \\
\text { All outcomes }\end{array}$ & Low risk & $\begin{array}{l}\text { Quote: "The examining dentist therefore at no time knew which toothbrush } \\
\text { was being tested by a subject," p. } 59 .\end{array}$ \\
\hline $\begin{array}{l}\text { Incomplete outcome data } \\
\text { (attrition bias) } \\
\text { All outcomes }\end{array}$ & Low risk & Comment: no missing data \\
\hline $\begin{array}{l}\text { Selective reporting (re- } \\
\text { porting bias) }\end{array}$ & Unclear risk & Comment: no protocol, but all listed outcomes were reported. \\
\hline
\end{tabular}

Dôgan 2004

Methods Study design: RCT - stratified randomisation, cross-over with 3 arms

Date of study: not stated, but prior to 2004 - "received for publication 1st October 2004"

Study duration: 5 weeks (including 2 weeks' washout) 
Dôgan 2004 (Continued)

Location: Turkey

Setting: school

Ethical approval: yes, p. 351

Consent: yes

Pescription of ID used: "mild mental disability," according to their IQ level, p. 351
Conversion to ICD description: mild
Age range (mean): 6-18 years (not reported)
Sex: not reported
Comorbidity reported/details: not reported
Number of participants at baseline: 30
Number of participants at final evaluation: unclear
Selection of the participants for the intervention: children attending the same class
Comparison: electric toothbrush versus manual toothbrush
Comparison: special manual toothbrush versus conventional manual toothbrush
Group 1: Oral B Braun electric toothbrush, with instruction
Group 2: Superbrush special manual toothbrush with instruction
Group 3: Oral B Cross Action manual toothbrush with instruction
Brushing time 3 minutes twice a day. All groups used same toothpaste throughout study.

Outcomes $\quad$ 1. Modified Quigley-Hein Plaque Index (V)

2. API (V)

Timing of outcome assessments: baseline and week 1, for each arm, with 1-week washout period

COM-B System Character- Potential sources of behaviour change: capability (physical), opportunity (physical) istics

Potential Intervention functions: training, environmental restructuring

\section{Stakeholder Involvement Formal or non-formal carer: unclear}

With or without dental professional involvement: with - dental profession unclear

Other stakeholders: unclear, no mention of who supervised brushing

Notes Strengths and weaknesses: data reported for the final arm only

Modifications to the intervention: none reported

Adverse effects: none reported

Funding source: "Thanks to Braun and Denta Co. AS for their free sample supplements."

\section{Risk of bias}

Bias Authors' judgement Support for judgement


Dôgan 2004 (Continued)

Random sequence genera- Unclear risk Quote: "The children were randomly assigned to three groups," p. 351. tion (selection bias)

Comment: no further details provided.

\begin{tabular}{lll}
\hline $\begin{array}{l}\text { Allocation concealment } \\
\text { (selection bias) }\end{array}$ & Unclear risk & Comment: no further details provided. \\
\hline $\begin{array}{l}\text { Blinding of participants } \\
\text { and personnel (perfor- } \\
\text { mance bias) }\end{array}$ & High risk & Comment: not possible to blind participants or personnel. \\
$\begin{array}{l}\text { All outcomes } \\
\text { Blinding of outcome as- } \\
\text { sessment (detection bias) } \\
\text { All outcomes }\end{array}$ & Unclear risk & $\begin{array}{l}\text { Quote "the same examiner, whose identity was concealed to the children," p. } \\
\end{array}$ \\
\end{tabular}

Incomplete outcome data Unclear risk

Comment: no details of dropouts reported.

(attrition bias)

All outcomes

Selective reporting (re- Unclear risk Comment: no protocol but all listed outcomes reported.
porting bias)

\section{Ferozali 2007}

\begin{tabular}{|c|c|}
\hline \multirow[t]{7}{*}{ Methods } & Study design: RCT - participants assigned to groups randomly \\
\hline & Date of study: not stated, but after 2000 and pre-2007. Quote: "In 2000 prior to the current study..." \\
\hline & Study duration: 90 days \\
\hline & Location: USA \\
\hline & Setting: residential \\
\hline & $\begin{array}{l}\text { Ethical approval: yes. Quote: "local and state institutional review board approval," p. 169. Comment: } \\
\text { assumed adequate. }\end{array}$ \\
\hline & Consent: yes \\
\hline \multirow[t]{8}{*}{ Participants } & Description of ID used: "developmentally disabled with profound mental retardation" \\
\hline & Conversion to ICD description: profound \\
\hline & Age range (mean): $31-79$ years (53.6 years) \\
\hline & Sex: male 26 , female 10 \\
\hline & $\begin{array}{l}\text { Comorbidity reported/details: quote: "Enteral feeding, hiatal hernia, emesis, gastro-oesophageal reflux } \\
\text { disease or swallowing problems" }\end{array}$ \\
\hline & Number of participants at baseline: 36 \\
\hline & Number of participants at final evaluation: 32 \\
\hline & $\begin{array}{l}\text { Selection of the participants for the intervention: dependent on nursing staff to carry out activities of } \\
\text { daily living }\end{array}$ \\
\hline Interventions & Comparison: special manual toothbrush versus conventional manual toothbrush \\
\hline
\end{tabular}


Ferozali 2007 (Continued)

Group 1: oral cleansing with a single-use suction toothbrush with sodium bicarbonate and a $1.5 \%$ hydrogen peroxide solution. Intermittent oral suctioning was given during the cleansing procedure.

Group 2: toothbrushing with a traditional toothbrush with sodium bicarbonate and a $1.5 \%$ hydrogen peroxide solution. Intermittent oral suctioning was provided as needed with a tonsil suction device during the cleansing procedure.

Group 3: oral cleansing with a traditional toothbrush and regular fluoride toothpaste was provided as before the study. Intermittent oral suctioning was provided as needed with a tonsil suction device during the cleansing procedure.

Group 1 and 2 only were eligible for inclusion in this review.

1. Oral health assessment using a simplified Beck Oral Assessment Tool - tongues, lips, oral mucosa
and gums
2. Bacterial load was assessed using oral cultures $(\mathrm{V})$
Timing of outcome assessments: 1. baseline, midway and day 90; 2. baseline and final

COM-B System Character- Potential sources of behaviour change: opportunity (physical)
istics

Potential Intervention functions: environmental restructuring

\begin{tabular}{ll} 
Stakeholder Involvement & $\begin{array}{l}\text { Formal or non-formal carer: formal } \\
\text { With or without dental professional involvement: with - dentist and dental hygienist } \\
\text { Other stakeholder involvement: nursing staff carried out the toothbrushing }\end{array}$ \\
\hline Notes & Strengths and weaknesses: quote: "All groups were brushed for one minute" twice daily, p. 170. \\
& Modifications to the intervention: none reported \\
& Adverse effects: quote: "Tested for allergies at outset." No adverse effects reported, p. 170. \\
& Funding source: quote: "Sage Products donated the suction brushes, but provided no additional fund- \\
ing (Sage Products, Inc., Cary, IL)," p. 169.
\end{tabular}

\section{Risk of bias}

\begin{tabular}{lll}
\hline Bias & Authors' judgement & Support for judgement \\
\hline $\begin{array}{l}\text { Random sequence genera- } \\
\text { tion (selection bias) }\end{array}$ & Low risk & $\begin{array}{l}\text { Quote: "Assignment to groups was determined by a computer-generated table } \\
\text { of purely random numbers," p. } 169 .\end{array}$ \\
\hline $\begin{array}{l}\text { Allocation concealment } \\
\text { (selection bias) }\end{array}$ & Low risk & $\begin{array}{l}\text { Comment: no details provided but probably done as randomisation process } \\
\text { was good. }\end{array}$ \\
\hline
\end{tabular}

Blinding of participants Unclear risk Comment: no details provided; teeth were brushed by carers.
and personnel (perfor-
mance bias)
All outcomes

\begin{tabular}{lll}
\hline $\begin{array}{l}\text { Blinding of outcome as- } \\
\text { sessment (detection bias) } \\
\text { All outcomes }\end{array}$ & Low risk & $\begin{array}{l}\text { Oral health - quote: "scored according to observations made by the dental hy- } \\
\text { gienist who was blinded to the group membership of the participants." Bacter- } \\
\text { ial load - quote: "investigator who was blinded to group membership," p. } 170 .\end{array}$ \\
\hline $\begin{array}{l}\text { Incomplete outcome data } \\
\text { (attrition bias) } \\
\text { All outcomes }\end{array}$ & Low risk & Comment: missing data fully explained. \\
\hline
\end{tabular}


Ferozali 2007 (Continued)

Selective reporting (re- Unclear risk Comment: no protocol but all listed outcomes reported. porting bias)

\begin{tabular}{|c|c|}
\hline \multirow[t]{7}{*}{ Methods } & Study design: cluster RCT - quote: "Cluster-randomised single-blinded (examiner) clinical trial" \\
\hline & Date of study: April 2014 \\
\hline & Study duration: 6 months \\
\hline & Location: Spain \\
\hline & Setting: school \\
\hline & $\begin{array}{l}\text { Ethical approval: quote: "The regional ethical committee approved (13/302-E, } 7 \text { August 2013) the in- } \\
\text { formed consents and the protocol," p. } 2 \text {. }\end{array}$ \\
\hline & Consent: yes \\
\hline \multirow[t]{8}{*}{ Participants } & $\begin{array}{l}\text { Description of ID used: "The mean IQ was } 60.6 \text {, ranging between } 44 \text { and } 87, " \text { p. } 4 \text {. "Only two categories } \\
\text { of ID were included, light ( } \mathrm{n} \text { [number of participants] = 54) and limit }(n=10), " \text { p. } 4 \text {. }\end{array}$ \\
\hline & Conversion to ICD description: mild and moderate \\
\hline & Age range (mean): $18-65$ years (34.5 years) \\
\hline & Sex: 34 male, 30 female \\
\hline & $\begin{array}{l}\text { Comorbidity reported/details: anxiety disorder, epilepsy, ocular tension, chronic bronchitis, asthma, } \\
\text { hepatitis C, hypertension and depression, right leg hemiparesis, type } 2 \text { diabetes, hypothyroidism, para- } \\
\text { noid schizophrenia, thyroid problems, p. } 4\end{array}$ \\
\hline & Number of participants at baseline: 64 \\
\hline & Number of participants at final evaluation: 60 \\
\hline & $\begin{array}{l}\text { Selection of the participants for the intervention: support groups (clusters) for those with limited or } \\
\text { moderate ID were recruited. }\end{array}$ \\
\hline
\end{tabular}

\section{Interventions Comparison: electric toothbrush versus manual toothbrush}

Both groups brushed for 2 minutes, monitored by a trained supervisor with written instructions, for the first 3 months.

Group 1: Sonicare, Philips electric toothbrush

Group 2: Vitis Access, manual toothbrush

Outcomes

Outcomes measured "were scored four sites per tooth in two randomly (by coin toss) selected quadrants (one in the upper jaw, one in the lower jaw, contralateral - Bentley \& Disney 1995."

1. Gingival Index (Löe 1963)

2. Plaque Index (Silness 1964)

3. Calculus - presence or absence

4. Presence of adverse effects was assessed by a visual inspection at each study visit

Timing of outcome assessments: baseline, 3 months, 6 months 
Garcia-Carrillo 2016 (Continued)

COM-B System Character- Potential sources of behaviour change: capability (physical/psychological), opportunity (physical) istics

Potential intervention functions: training, persuasion, environmental restructuring

Stakeholder Involvement Formal or non-formal carer: formal

With or without dental professional involvement: without

Other stakeholder involvement: trained monitor ("special educators, with different university degrees in pedagogy"), p. 2.

Notes

Strengths and weaknesses: quote: "The obtained results, therefore, should be interpreted with caution and only should be extrapolated to populations with mild ID and fine motor skills," p. 9.

Modifications to the intervention: none reported

Adverse effects: none reported from either toothbrush, some bleeding related to periodontal status, $\mathrm{p}$. 5 .

Funding source: quote: "This study was supported by an unrestricted grant from the Phillips Oral Healthcare, by means of a research contract (362/2013) with University Complutense."

\section{Risk of bias}

\begin{tabular}{|c|c|c|}
\hline Bias & Authors' judgement & Support for judgement \\
\hline $\begin{array}{l}\text { Random sequence genera- } \\
\text { tion (selection bias) }\end{array}$ & Low risk & Quote: "a computer-generated randomization list, by an external agent," p. 2. \\
\hline $\begin{array}{l}\text { Allocation concealment } \\
\text { (selection bias) }\end{array}$ & Low risk & $\begin{array}{l}\text { Quote: "a computer-generated randomization list, by an external agent," p. } 2 . \\
\text { Comment: allocation concealment probably achieved. }\end{array}$ \\
\hline $\begin{array}{l}\text { Blinding of participants } \\
\text { and personnel (perfor- } \\
\text { mance bias) } \\
\text { All outcomes }\end{array}$ & Unclear risk & $\begin{array}{l}\text { Comment: unclear if personnel were blinded; study was a cluster study so par- } \\
\text { ticipants were most likely blinded. }\end{array}$ \\
\hline $\begin{array}{l}\text { Blinding of outcome as- } \\
\text { sessment (detection bias) } \\
\text { All outcomes }\end{array}$ & Low risk & $\begin{array}{l}\text { Quote: "by a single calibrated and trained examiner, blinded to the group allo- } \\
\text { cation." "The clinical evaluators were not aware of the group assignment or in- } \\
\text { volved in the randomization process," p. } 3 \text {. }\end{array}$ \\
\hline $\begin{array}{l}\text { Incomplete outcome data } \\
\text { (attrition bias) } \\
\text { All outcomes }\end{array}$ & Low risk & $\begin{array}{l}\text { Comment: all outcomes reported, detailed levels of reporting, low levels of at- } \\
\text { trition; reasons for loss not related to outcomes. }\end{array}$ \\
\hline $\begin{array}{l}\text { Selective reporting (re- } \\
\text { porting bias) }\end{array}$ & Unclear risk & Comment: no protocol but all listed outcomes reported. \\
\hline
\end{tabular}


Gertenrich 1967a (Continued)

\author{
Setting: residential \\ Ethical approval: not reported \\ Consent: not reported
}

Participants

Description of ID used: "Cerebral palsied patients and mentally retarded but trainable patients" with IQ ranging from 30 to 95 for people with cerebral palsy and 30 to 50 for people with mental retardation, $p$. 155.

Conversion to ICD description: mixed with some IQ > 70

Age range: $15-30$ years

Sex: 30 male, 6 female

Comorbidity reported/details: cerebral palsy

Number of participants at baseline: 39

Number of participants at final evaluation: 38

Selection of the participants for the intervention: no details given

\title{
Interventions Comparison: electric toothbrush versus manual toothbrush
}

Group 1a: cerebral palsy unit - attendants brushed with Oral B Automatic Toothbrush Arcuate actions electric toothbrush

Group 1b: cerebral palsy unit - attendants brushed with a manual toothbrush

Group 2a: "trainable" unit - attendants brushed with Oral B Automatic Toothbrush Arcuate actions electric toothbrush

Group 2b: "trainable" unit - self-brushed with Oral B Automatic Toothbrush Arcuate actions electric toothbrush

Group 2c: "trainable" unit - attendants brushed with a manual toothbrush

Group 2d: "trainable" unit - self-brushed with a manual toothbrush

IQ: Group 1 30-95; Group 2 30-50

We combined data from self-brushers and attendant brushed participants for each brush type, for analysis.

Outcomes

1. Oral hygiene categories: good, fair, poor and very poor: 1-4, higher scores are poorer (UV)

2. Gingival Inflammation categories observed: absent, obvious gingivitis, decrease in severity (UV)

3. Some subjective feedback from attendants (UV)

Timing of outcome assessments: baseline, weekly for 8 weeks, final at week 8 and follow-up at week 20 (reported 3)

COM-B System Characteristics
Potential sources of behaviour change: capability (physical), opportunity (physical), motivation (reflective)

Potential intervention functions: training, enablement, coercion (for carers only), environmental restructuring

\section{Stakeholder Involvement Formal or non-formal carer: formal}

With or without dental professional involvement: with - dentist and dental hygienists 
Other stakeholder involvement: attendants who brushed the participants' teeth

Notes Strengths and weaknesses: multiple modifications to the intervention

Modifications to the intervention: in the "trainable units" using the electric toothbrush, both attendant brushed and self-brushers returned to using manual brushes for the follow-up period. The improvements in both the cerebral palsy groups occurred "due to the direct supervision by members of the institution's nursing staff," p. 159.

Adverse effects: none reported

Funding source: none reported

\section{Risk of bias}

\begin{tabular}{|c|c|c|}
\hline Bias & Authors' judgement & Support for judgement \\
\hline $\begin{array}{l}\text { Random sequence genera- } \\
\text { tion (selection bias) }\end{array}$ & High risk & Comment: no mention of randomisation. \\
\hline $\begin{array}{l}\text { Allocation concealment } \\
\text { (selection bias) }\end{array}$ & Unclear risk & Comment: no details given. \\
\hline $\begin{array}{l}\text { Blinding of participants } \\
\text { and personnel (perfor- } \\
\text { mance bias) } \\
\text { All outcomes }\end{array}$ & Unclear risk & Comment: no details given. \\
\hline $\begin{array}{l}\text { Blinding of outcome as- } \\
\text { sessment (detection bias) } \\
\text { All outcomes }\end{array}$ & Low risk & $\begin{array}{l}\text { Comment: assessors did not know to which group the subjects were assigned, } \\
\text { p. } 147 .\end{array}$ \\
\hline $\begin{array}{l}\text { Incomplete outcome data } \\
\text { (attrition bias) } \\
\text { All outcomes }\end{array}$ & Low risk & $\begin{array}{l}\text { Comment: } 1 \text { dropout. No reason given, but unlikely to have affected out- } \\
\text { comes. }\end{array}$ \\
\hline $\begin{array}{l}\text { Selective reporting (re- } \\
\text { porting bias) }\end{array}$ & Unclear risk & Comment: no protocol but all listed outcomes reported. \\
\hline
\end{tabular}

\section{Gertenrich 1967b}

\begin{tabular}{ll} 
Methods & Study design: NRCT - quote: "children were divided into two groups," p. 145. \\
& Date of study: not reported, pre-1967 \\
& Study duration: $21-28$ weeks \\
& Location: USA \\
& Setting: residential \\
& Ethical approval: not reported \\
& Consent: not reported \\
& Description of ID used: "Mongoloid and others possessing an average IQ of 20." Later described as an IQ \\
from 0 to 20. & Conversion to ICD description: severe or profound \\
\hline Participants &
\end{tabular}


Gertenrich 1967b (Continued)

Age range: $5-25$ years

Sex: males 52, females 18 (Table 1, p. 146)

Comorbidity reported/details: quote: "requiring intensive care," No other details reported.

Number of participants at baseline: 75 (Table 2 and 3, p. 146 - numbers did not match Table 1).

Number of participants at final evaluation: 72

Selection of the participants for the intervention: no details reported

Interventions

\section{Comparison: electric toothbrush versus manual toothbrush}

Group 1: participants with Down's syndrome had teeth brushed twice a day with an Oral B Automatic Toothbrush - Arcuate actions by attendants who were told oral hygiene would be monitored after initial 8 weeks.

Group 2: participants with Down's syndrome had teeth brushed twice a day with manual toothbrush by attendants who were told oral hygiene would be monitored.

Group 3: participants with "low IQ" had teeth brushed twice a day with an Oral B Automatic Toothbrush - Arcuate actions by attendants who were told the intervention was finished and oral hygiene would be not be monitored after initial 8 weeks.

Group 4: participants with "low IQ" had teeth brushed twice a day with a manual toothbrush by attendants who were told the intervention was finished and oral hygiene would be not be monitored after initial 8 weeks.

We combined data for the participants with Down's syndrome and "low IQ" for the toothbrush types, for analysis.

Outcomes

1. Oral hygiene categories: good, fair, poor and very poor: 1-4, higher scores were poorer (UV)

2. Gingival inflammation categories observed: absent, obvious gingivitis, decrease in severity (UV)

3. Some subjective feedback from attendants (UV)

Timing of outcome assessments: 1 . weekly, for 20-28 weeks, 2. baseline, 8-10 weeks, and 20-28 weeks

COM-B System Characteristics

Potential sources of behaviour change: opportunity (physical), motivation (reflective)

Potential intervention functions: coercion, environmental restructuring

Stakeholder Involvement Formal or non-formal carer: formal

With or without dental professional involvement: with - dentist and dental hygienists

Other stakeholder involvement: attendants brushed the participants' teeth

Notes Strengths and weaknesses: subjective measure of oral hygiene and gingival inflammation.

Modifications to the intervention: unclear; the interval of follow-up may have been extended due to the outbreak of mumps and measles in 2 groups.

Adverse effects: unclear - authors refer to "apparent disadvantages" of using an electric toothbrush, but it is unclear if these disadvantages arose in this study," p. 150.

Funding source: none reported

\section{Risk of bias}


Gertenrich 1967b (Continued)

Random sequence genera- High risk Comment: no report of randomisation - participants were divided equally, tion (selection bias) with equal numbers of males and females in each group.

Allocation concealment $\quad$ Unclear risk $\quad$ Comment: no details provided.
(selection bias)

Blinding of participants Unclear risk Comment: no details provided.
and personnel (perfor-
mance bias)
All outcomes

\begin{tabular}{|c|c|c|}
\hline $\begin{array}{l}\text { Blinding of outcome as- } \\
\text { sessment (detection bias) } \\
\text { All outcomes }\end{array}$ & Low risk & $\begin{array}{l}\text { Comment: assessors did not know to which group participants were assigned, } \\
\text { p. } 147 .\end{array}$ \\
\hline
\end{tabular}

Incomplete outcome data Unclear risk Comment: 3 dropouts but no reasons given. Unlikely to affect outcome.
(attrition bias)

All outcomes

\begin{tabular}{l}
\hline $\begin{array}{l}\text { Selective reporting (re- } \\
\text { porting bias) }\end{array}$ \\
\hline
\end{tabular}

Gertenrich 1967c

Methods
Study design: NRCT. Quote: "Each group were divided into two equal sub groups," p. 151.
Study duration: 34 weeks
Location: USA
Setting: residential
Ethical approval: not reported
Consent: not reported
Description of ID used: IQ range 0-20 (Level V AAMD) "Only a few could talk and none was self-fed or toi-
let-trained," p. 150.
Conversion to ICD description: severe to profound
Age range: 2 -12 years
Sex: male 24, female 24
Comorbidity reported/details: all non-ambulatory, p. 150
Number of participants at baseline: 48
Number of participants at final evaluation: 48
Selection of the participants for the intervention: "selected at random," p. 150

Interventions

\section{Comparison: electric toothbrush versus manual toothbrush}

All attendants were given training in toothbrushing techniques. 
Group 1: hydrocephaly and seizure unit participants had their teeth brushed by the attendants, using an "Oral B Automatic Toothbrush - Arcuate actions" electric toothbrush.

Group 2: hydrocephaly and seizure unit participants had their teeth brushed by the attendants, using a manual toothbrush.

Group 3: paediatric unit participants had their teeth brushed by the attendants, using an "Oral B Automatic Toothbrush - Arcuate actions" electric toothbrush.

Group 4: paediatric unit participants had their teeth brushed by the attendants, using manual toothbrush.

We combined data from the hydrocephaly and seizure units and from the paediatric units were combined for each toothbrush type, for analysis.

1. Oral hygiene categories: good, fair, poor and very poor: 1-4, higher scores were poorer (UV)
2. Gingival inflammation categories observed: absent, obvious gingivitis, decrease in severity (UV)
3. Some subjective feedback from attendants (UV)
Timing of outcome assessments: baseline, weekly for 8 weeks, follow-up 20-34 weeks. Reported 0, 8 ,
20 and 34 weeks

COM-B System Characteristics
Potential sources of behaviour change: capability (physical), opportunity (physical), motivation (reflective)

Potential intervention functions: training, enablement, coercion, environmental restructuring

\begin{tabular}{|c|c|c|}
\hline \multirow[t]{4}{*}{ Notes } & \multicolumn{2}{|c|}{$\begin{array}{l}\text { Strengths and weaknesses: no effort made to certify that each participant was brushed twice daily as } \\
\text { instructed. Researcher suspected it was not happening in some groups. }\end{array}$} \\
\hline & \multicolumn{2}{|c|}{$\begin{array}{l}\text { Modifications to the intervention: findings of the study were discussed with groups } 1 \text { and } 2 \text { at } 20 \text { weeks } \\
\text { due to the attendants failing to brush twice daily or even daily in these groups. This resulted in changes } \\
\text { to the organisation of the attendants, which may have impacted on the } 28 \text {-week results, p. } 156 \text {. }\end{array}$} \\
\hline & \multicolumn{2}{|c|}{ Adverse effects: none reported } \\
\hline & \multicolumn{2}{|c|}{ Funding source: none reported } \\
\hline \multicolumn{3}{|l|}{ Risk of bias } \\
\hline Bias & Authors' judgement & Support for judgement \\
\hline $\begin{array}{l}\text { Random sequence genera- } \\
\text { tion (selection bias) }\end{array}$ & High risk & Comment: no reported randomisation - groups divided equally. \\
\hline $\begin{array}{l}\text { Allocation concealment } \\
\text { (selection bias) }\end{array}$ & Unclear risk & Comment: no details given. \\
\hline $\begin{array}{l}\text { Blinding of participants } \\
\text { and personnel (perfor- } \\
\text { mance bias) } \\
\text { All outcomes }\end{array}$ & Unclear risk & Comment: no details given. \\
\hline
\end{tabular}


Gertenrich 1967c (Continued)

Blinding of outcome as- Low risk Comment: assessors did not know to which group the participants were assessment (detection bias) $\quad$ signed, p. 147.

All outcomes

$$
\text { signed, p. } 147 .
$$

Incomplete outcome data Low risk Comment: no missing data.
(attrition bias)

All outcomes

Selective reporting (re- Unclear risk $\quad$ Comment: no protocol but all listed outcomes reported.

porting bias)

Gertenrich 1967d

\begin{tabular}{ll}
\hline Methods & Study design: NRCT \\
Date of study: not stated, pre-1967 \\
Study duration: 24 weeks \\
Location: USA \\
Setting: residential \\
Ethical approval: not reported \\
Consent: not reported
\end{tabular}

Participants

Description of ID used: "mildly retarded patients, educable group, classed as "slow learners."

Conversion to ICD description: mild or IQ > 70

Age range (mean): 13-18 years

Sex: 22 male, 22 female

Comorbidity reported/details: none reported

Number of participants at baseline: 43

Number of participants at final evaluation: 41

Selection of the participants for the intervention: no details reported

Interventions

\section{Comparison: electric toothbrush versus manual toothbrush}

"Attendants were trained in the proper techniques and provided supervision and assistance," p. 161.

Group 1: self-brushed with an Oral B Automatic Toothbrush Arcuate actions electric toothbrush

Group 2: self-brushed with a manual toothbrush

1. Oral hygiene categories: good, fair, poor and very poor: 1-4, higher scores were poorer (UV)
2. Gingival Inflammation - categories observed: absent, obvious gingivitis, decrease in severity (UV)
Timing of outcome assessments: baseline, 8 weeks and 24 weeks. No report of the weekly assessment
from 0 to 8 weeks but it did appear to have been recorded in the graphs.

\footnotetext{
COM-B System Characteristics

Potential sources of behaviour change: capability (physical/psychological), opportunity (physical)
} 
Gertenrich 1967d (Continued)

Potential intervention functions: training, enablement, persuasion, environmental restructuring

Stakeholder Involvement $\quad$ Formal or non-formal carer: formal

With or without dental professional involvement: with - dentist and dental hygienists

Other stakeholder involvement: attendants who supervised and assisted

Notes

Strengths and weaknesses: IQ level of some participants in this study may have been $>70$

Modifications to the intervention: none reported

Adverse effects: none reported

Funding source: none reported

\section{Risk of bias}

\begin{tabular}{|c|c|c|}
\hline Bias & Authors' judgement & Support for judgement \\
\hline $\begin{array}{l}\text { Random sequence genera- } \\
\text { tion (selection bias) }\end{array}$ & High risk & Comment: no evidence of randomisation. \\
\hline $\begin{array}{l}\text { Allocation concealment } \\
\text { (selection bias) }\end{array}$ & Unclear risk & Comment: no details provided. \\
\hline $\begin{array}{l}\text { Blinding of participants } \\
\text { and personnel (perfor- } \\
\text { mance bias) } \\
\text { All outcomes }\end{array}$ & Unclear risk & Comment: no details provided. \\
\hline $\begin{array}{l}\text { Blinding of outcome as- } \\
\text { sessment (detection bias) } \\
\text { All outcomes }\end{array}$ & Low risk & $\begin{array}{l}\text { Comment: assessors did not know to which group the participants were as- } \\
\text { signed, p. } 147 .\end{array}$ \\
\hline $\begin{array}{l}\text { Incomplete outcome data } \\
\text { (attrition bias) } \\
\text { All outcomes }\end{array}$ & Low risk & $\begin{array}{l}\text { Comment: } 2 \text { dropouts, } 1 \text { from each group. No reason given, but unlikely to } \\
\text { have impacted on outcomes. }\end{array}$ \\
\hline $\begin{array}{l}\text { Selective reporting (re- } \\
\text { porting bias) }\end{array}$ & Unclear risk & Comment: no protocol but all listed outcomes reported. \\
\hline
\end{tabular}

Glassman 2006

Study design: NRS - "multiple baseline design"
Date of study: not given, pre-2006
Study duration: 3-hour training session with trainer/observer providing feedback for 142-156 days,
plus telephone call follow-up 1 month after study end.
Location: USA
Setting: residential
Ethical approval: none reported
Consent: quote: "Informed consent documents were obtained for all participants," p. 29.


Glassman 2006 (Continued)

Participants
Description of ID used: "with developmental disabilities," p. 39. "Mild to moderate mental retardation," p. 45. Moderate, severe (Table 1).

Conversion to ICD description: mild, moderate and severe

Age range: participants $20-54$ years, carers: $27-54$ years

Sex: participants: 8 female, 3 male; carers: 7 female, 3 male

Comorbidity reported/details: yes, blind, deaf, heart defect, seizures, cerebral palsy

Number of participants at baseline: 11 participants; 10 carers

Number of participants at final evaluation:11 participants; 10 carers

Selection of the participants for the intervention: selected to participate carers interested in improving their knowledge of preventive dentistry, p. 39.
Interventions

\section{Comparison: training of carer versus no training of carer}

Carers received $2 \times 3$-hour classes in preventive dentistry and basic applied behavioural principles using a training package "Overcoming obstacles to Dental Health," p. 41. Field observers provided ongoing verbal feedback and made supportive suggestions during the intervention. Toothbrushing sessions were videotaped. 3 settings.

Outcomes 1. Social validation of client's abilities with regard to oral hygiene - 8 questions on 6 -point scale completed by carers.

2. Carers' perception of training - 6 open-ended questions via telephone

3. Plaque score

4. Carers' presence during toothbrushing

5. Observed $\%$ of tooth surfaces brushed

6. Duration of brushing

Timing of outcome assessments: 1 . baseline, final; 2. 1-month follow-up; outcomes 3-6 - assumed daily

Details and tables unclear. Details requested from authors (no response).

COM-B System Characteristics
Potential sources of behaviour change: carer: capability (physical/psychological), opportunity (social/physical), motivation (reflective). People with ID: capability (physical), opportunity (physical), motivation (reflective)

Potential intervention functions: carers: education, training, enablement, incentivisation, environmental restructuring; people with ID: training, modelling, persuasion

Stakeholder Involvement Formal or non-formal carer: formal

With or without dental professional involvement: with - dental assistant, dental hygienist

Other stakeholder involvement: carers

Notes

Strengths and weaknesses: quote: "The issue of caregiver participation was problematic for the researchers throughout the project," p. 45. Carers received compensation for their participation; no conflict likely. Intervention developers appeared to have evaluated their own intervention.

Modifications to the intervention: a specific instruction to the carers to apply the training in practice was added to the intervention, p. 44.

Adverse effects: none reported. 


\section{Risk of bias}

Bias Authors' judgement Support for judgement

\begin{tabular}{|c|c|c|}
\hline $\begin{array}{l}\text { Was the intervention inde- } \\
\text { pendent of other changes? }\end{array}$ & Unclear risk & $\begin{array}{l}\text { Comment: no details provided. Length of intervention made it possible that } \\
\text { other changes had occurred. }\end{array}$ \\
\hline
\end{tabular}
(ITS)

Comment: no details provided. Length of intervention made it possible that

Comment: the point of analysis was the point of intervention. The direction of the outcomes was clearly stated, pp. 40-1.

\begin{tabular}{|c|c|c|}
\hline $\begin{array}{l}\text { Was the shape of the in- } \\
\text { tervention effect prespeci- } \\
\text { fied? (ITS) }\end{array}$ & Low risk & $\begin{array}{l}\text { Comment: the point of analysis was the point of intervention. The direction of } \\
\text { the outcomes was clearly stated, pp. } 40-1 .\end{array}$ \\
\hline
\end{tabular}
fied? (ITS)

\section{Was the intervention un- likely to affect data collec- tion? (ITS)}

Low risk Comment: the intervention did not affect either the source or method of data collection.

Was knowledge of the al- High risk Comment: not possible to blind participants or personnel.
located interventions ade-
quately prevented during
the study? (ITS)

\begin{tabular}{lll}
\hline $\begin{array}{l}\text { Were incomplete out- } \\
\text { come data adequately ad- } \\
\text { dressed? (ITS) }\end{array}$ & Low risk & \\
\hline $\begin{array}{l}\text { Was the study free from } \\
\text { selective outcome report- }\end{array}$ & Unclear risk & Comment: poor image quality of data table 1. \\
ing? (ITS) & & \\
\hline
\end{tabular}

\section{Gonzalez 2013}

Study design: RCT
Date of study: no details given, pre-2003
Study duration: 30 or 90 minutes
Location: USA
Setting: residential, p. 293
Ethical approval: expedited approval was obtained from the Human Research Review Committee
(HRRC) at the University of New Mexico prior to the initiation of this study. Study and protocol number
HRRC\#: 09-456," p. 294.
Consent: yes - "After consent forms were obtained," p. 294.
Description of ID used: "developmental disabilities"
Conversion to ICD description: unclear
Age range: not reported
Sex: male 8, female 14, not reported for 2 participants
Comorbidity reported/details: none reported


Number of participants at baseline: 30

Number of participants at final evaluation: 24

Selection of the participants for the intervention: a convenient sample

Interventions Comparison: training of carers versus no training of carers

Group 1: 90-minute lecture and hands-on seminar were presented covering topics in oral health for people with developmental disabilities including tooth brushing techniques, plaque removal, progression of periodontal disease and techniques on how to approach a patient with challenging behaviour when performing oral hygiene

Group 2: 30-minute discussion among the participants on familiar topics

Outcomes 1 Knowledge and comprehension regarding oral health topics - 20 questions

Timing of outcome assessments: immediately pre- and postintervention

COM-B System Character- Potential sources of behaviour change: capability (physical/psychological), opportunity (social/physiistics cal), motivation (reflective)

Potential intervention functions: education, training, modelling, enablement

Stakeholder Involvement Formal or non-formal carer: formal

With or without dental professional involvement: with - dental hygienist

Other stakeholder involvement: none reported

Notes Strengths and weaknesses: existing knowledge high. Confusion regarding answer options and venue. Validity of tool is unclear; 2 different questionnaires were used for baseline and final.

Modifications to the intervention: none reported

Adverse effects: levels of stress and fatigue completing the questionnaire was assessed, none reported;

"No harms were expressed by any of the participants at the conclusion of the study," p. 295.

Funding source: caregivers received compensation for participation; no likely conflict of interest.

\section{Risk of bias}

Bias Authors' judgement Support for judgement

Random sequence genera- Low risk tion (selection bias)
Quote: "Enrolment was established on voluntary participation and assigning participants randomly to either group A (experimental) or group B (control) based on phone calls. Random assignment of the research subjects to one of two sites was completed by the health coordinator/recruiter of ARCA," p. 294.

Comment: probably done

Allocation concealment Low risk
(selection bias)

Allocation concealment Low risk

Quote: "Enrolment was established on voluntary participation and assigning participants randomly to either group A (experimental) or group B (control) based on phone calls. Random assignment of the research subjects to one of two sites was completed by the health coordinator/recruiter of ARCA," p. 294.

Comment: probably done

$\begin{array}{ll}\begin{array}{l}\text { Blinding of participants } \\ \text { and personnel (perfor- }\end{array} & \text { Low risk } \\ \begin{array}{l}\text { mance bias) } \\ \text { All outcomes }\end{array} & \begin{array}{l}\text { Comment: both participants and recruiter were blinded as to which group was } \\ \text { experimental or control. The study took place at } 2 \text { different ARCA locations the } \\ \text { same day and same time, p. } 294 .\end{array}\end{array}$


Gonzalez 2013 (Continued)

Blinding of outcome as- Unclear risk Comment: no report of blinding. sessment (detection bias)

All outcomes

\begin{tabular}{|c|c|c|}
\hline $\begin{array}{l}\text { Incomplete outcome data } \\
\text { (attrition bias) } \\
\text { All outcomes }\end{array}$ & Low risk & $\begin{array}{l}\text { Comment: reason for missing data is not related to the outcomes. } \\
\text { Quote: "Two participants showed up to wrong location, one participant did } \\
\text { not complete tests accurately and two others did not show up to the study." }\end{array}$ \\
\hline $\begin{array}{l}\text { Selective reporting (re- } \\
\text { porting bias) }\end{array}$ & Unclear risk & Comment: no protocol but all listed outcomes reported. \\
\hline
\end{tabular}

\section{Jarman 1983}

Study design: ITS
Date of study: not stated, pre-1983
Study duration: $29-30$ weeks
Location: USA
Setting: residential
Ethical approval: not reported
Consent: not reported

\section{Participants}

Description of ID used: "moderately to severely mentally retarded"

Conversion to ICD description: severe

Age range (mean): $14-57$ years (21.5 years)

Sex: mixed

Comorbidity reported/details: yes, cerebral palsy, all but 2 required a wheel chair or upright walker

Number of participants at baseline: 40

Number of participants at final evaluation: 40

Selection of the participants for the intervention: participants were living on the same unit in a residential facility.

Interventions

\section{Comparison: training for people with ID versus no training of people with ID}

Development of 6 skills in a morning personal care routine, of which only 1 skill, the toothbrushing skill, was relevant.

Group 1: use of reinforcement procedures; token reinforcement programme, single response contingency and chained response contingency, in developing a toothbrushing routine, p. 114.

Group 2: no training

1. Performance of the skill at each stage of training
2. Improvement scores for individuals


Jarman 1983 (Continued)

Timing of outcome assessments: daily, reported as mean at week 7 (baseline), week 25 (single contingency) and week 29 (chained contingency)

$\begin{array}{ll}\begin{array}{l}\text { COM-B System Character- } \\ \text { istics }\end{array} & \begin{array}{l}\text { Potential sources of behaviour change: capability (physical), opportunity (physical), motivation (reflec } \\ \text { tive) }\end{array} \\ & \begin{array}{l}\text { Potential intervention functions: training, enablement, persuasion, incentivisation, environmental re- } \\ \text { structuring }\end{array}\end{array}$

Stakeholder Involvement Formal or non-formal carer: formal

With or without dental professional involvement: without

Other stakeholder involvement: unit director, supervisors, attendants

Notes

Strengths and weaknesses: "...intensive instruction had been provided to each resident prior to the beginning of the study and all residents exhibited some initial competence in each skill," p. 119. "individual performance criteria was seen as an essential consideration," pp. 119-20.

Modifications to the intervention: none reported

Adverse effects: none reported

Funding source: "The research was supported in part by Gran \#00917-15-0 from the Maternal and Child Health Service."

\section{Risk of bias}

\begin{tabular}{|c|c|c|}
\hline Bias & Authors' judgement & Support for judgement \\
\hline $\begin{array}{l}\text { Was the intervention inde- } \\
\text { pendent of other changes? } \\
\text { (ITS) }\end{array}$ & High risk & $\begin{array}{l}\text { Comment: training method was being used for } 5 \text { other personal skills at the } \\
\text { same time. }\end{array}$ \\
\hline $\begin{array}{l}\text { Was the shape of the in- } \\
\text { tervention effect prespeci- } \\
\text { fied? (ITS) }\end{array}$ & Low risk & Comment: the point of analysis was the point of intervention. \\
\hline $\begin{array}{l}\text { Was the intervention un- } \\
\text { likely to affect data collec- } \\
\text { tion? (ITS) }\end{array}$ & Low risk & $\begin{array}{l}\text { Comment: the intervention did not affect either the source or method of data } \\
\text { collection. }\end{array}$ \\
\hline $\begin{array}{l}\text { Was knowledge of the al- } \\
\text { located interventions ade- } \\
\text { quately prevented during } \\
\text { the study? (ITS) }\end{array}$ & High risk & Comment: not possible to blind. \\
\hline $\begin{array}{l}\text { Were incomplete out- } \\
\text { come data adequately ad- } \\
\text { dressed? (ITS) }\end{array}$ & Low risk & Comment: no incomplete data. \\
\hline $\begin{array}{l}\text { Was the study free from } \\
\text { selective outcome report- } \\
\text { ing? (ITS) }\end{array}$ & Unclear risk & Comment: no protocol but all listed outcomes reported. \\
\hline
\end{tabular}

\section{Kaschke 2005}

Methods Study design: RCT 3-way cross-over trial


Date of study: not stated, prior to 2004 (thesis by Zeller 2004)

Study duration: 2 weeks for each arm, with 2-week washouts, 12 weeks in total

Location: Germany

Setting: unclear

Ethical approval: yes

Consent: not reported

\section{Participants}

Description of ID used: "varying degrees of intellectual and/or physical impairments" - author confirmed by email that all participants had an ID.

Conversion to ICD description: mixed

Age range (mean): $18-45$ years

Sex: not reported

Comorbidity reported/details: "varying degrees of intellectual and/or physical impairments," p. 66.

Number of participants at baseline: 36

Number of participants at final evaluation: 36

Selection of the participants for the intervention: all attended a university clinic for patients with special needs.

\section{Comparison: electric toothbrush versus manual toothbrush}

\section{Comparison: special manual toothbrush versus a conventional manual toothbrush}

Group 1: Teledyne Waterpik Sonic Toothbrush, with verbal and written instruction

Group 2: Superbrush special manual toothbrush with verbal and written instruction

Group 3: Oral B Cross manual toothbrush with verbal and written instruction

The same toothpaste was used by all groups.

Outcomes $\begin{aligned} & \text { 1. Modified Quigley-Hein Plaque Index (V) } \\ & \text { 2. API (V) } \\ & \text { 3. Papilla Bleeding Index (V) } \\ & \text { Timing of outcome assessments: week } 2 \text { for each arm }\end{aligned}$

COM-B System Character- Potential sources of behaviour change: capability (physical), opportunity (physical) istics

Potential intervention functions: training, enablement, environmental restructuring

$$
\begin{array}{ll}
\text { Stakeholder Involvement } & \text { Formal or non-formal carer: both } \\
& \text { With or without dental professional involvement: with - dentist } \\
& \text { Other stakeholder involvement: none reported }
\end{array}
$$


Kaschke 2005 (Continued)

Adverse effects: reported - fear/uncertainty in relation to the noise/movement of the electric toothbrush, vibration of electric toothbrush cause participants to spasm resulting in breakage of electric toothbrush (thesis by Zeller 2004).

Funding source: none reported

\section{Risk of bias}

\begin{tabular}{|c|c|c|}
\hline Bias & Authors' judgement & Support for judgement \\
\hline $\begin{array}{l}\text { Random sequence genera- } \\
\text { tion (selection bias) }\end{array}$ & Unclear risk & Comments: no details provided. \\
\hline $\begin{array}{l}\text { Allocation concealment } \\
\text { (selection bias) }\end{array}$ & Unclear risk & $\begin{array}{l}\text { Quote: "blind study" } \\
\text { Comment: unclear who was blinded. }\end{array}$ \\
\hline $\begin{array}{l}\text { Blinding of participants } \\
\text { and personnel (perfor- } \\
\text { mance bias) } \\
\text { All outcomes }\end{array}$ & High risk & Comment: cross-over trial, not possible to blind participants or personnel. \\
\hline $\begin{array}{l}\text { Blinding of outcome as- } \\
\text { sessment (detection bias) } \\
\text { All outcomes }\end{array}$ & Low risk & $\begin{array}{l}\text { Quote: "All examinations of the oral hygiene status were conducted by one ex- } \\
\text { aminer who had no knowledge about the toothbrush used by each subject," p. } \\
67 .\end{array}$ \\
\hline $\begin{array}{l}\text { Incomplete outcome data } \\
\text { (attrition bias) } \\
\text { All outcomes }\end{array}$ & Low risk & Comment: no missing data. \\
\hline $\begin{array}{l}\text { Selective reporting (re- } \\
\text { porting bias) }\end{array}$ & Unclear risk & $\begin{array}{l}\text { Comment: no protocol but all listed outcomes reported; no baseline data re- } \\
\text { ported. }\end{array}$ \\
\hline
\end{tabular}

Kelner 1963

\begin{tabular}{ll}
\hline Methods & Study design: RCT \\
Date of study: not provided, pre-1963 & Study duration: 4 months \\
Location: USA & Setting: school, day centre \\
& Ethical approval: not reported \\
& Consent: yes \\
\hline Description of ID used: "mental retardates" \\
Conversion to ICD description: unclear \\
Age range (mean): 4-32 years \\
Sex: not reported \\
Comorbidity reported/details: not reported \\
Number of participants at baseline: 108 \\
\hline
\end{tabular}


Kelner 1963 (Continued)

Number of participants at final evaluation: 100

Selection of the participants for the intervention: participants were enrolled in a nursery or work training centre sponsored by a local service organisation.

Interventions

\section{Comparison: electric toothbrush versus manual toothbrush}

Group 1: automated electric toothbrush (Broxodent) with instruction

Group 2: conventional toothbrush with instruction

\begin{tabular}{ll}
\hline Outcomes & 1. Hygiene deposits (subjective) (UV) \\
2. Gingival condition (subjective) (UV) \\
3. General evaluation (UV) \\
Timing of outcome assessments: baseline, month 4
\end{tabular}

COM-B System Character- Potential sources of behaviour change: capability (physical), opportunity (physical) istics

Potential Intervention functions: education, training, enablement, environmental restructuring

\section{Stakeholder Involvement Formal or non-formal carer: non-formal}

With or without dental professional involvement: with - dentist and dental student

Other stakeholder involvement: none reported

Strengths and weaknesses: use of subjective assessments
Modifications to the intervention: none reported
Adverse effects: none reported
Funding source: Broxodent supplied by ER Squibb and Sons (manufacturer)

\section{Risk of bias}

\begin{tabular}{|c|c|c|}
\hline Bias & Authors' judgement & Support for judgement \\
\hline $\begin{array}{l}\text { Random sequence genera- } \\
\text { tion (selection bias) }\end{array}$ & Unclear risk & $\begin{array}{l}\text { Quote: "selections were made at random," p. 103. No details of randomisation } \\
\text { process. }\end{array}$ \\
\hline $\begin{array}{l}\text { Allocation concealment } \\
\text { (selection bias) }\end{array}$ & Unclear risk & Comment: no details provided. \\
\hline $\begin{array}{l}\text { Blinding of participants } \\
\text { and personnel (perfor- } \\
\text { mance bias) } \\
\text { All outcomes }\end{array}$ & Low risk & $\begin{array}{l}\text { Comment: some of the participants in both groups had their teeth brushed by } \\
\text { their parents (no details on how many); both groups given } \mathrm{OHI} \text {. }\end{array}$ \\
\hline $\begin{array}{l}\text { Blinding of outcome as- } \\
\text { sessment (detection bias) } \\
\text { All outcomes }\end{array}$ & Low risk & $\begin{array}{l}\text { Quote: groups..."were made known to the examiner upon the completion of } \\
\text { the follow-up examination," p. } 103 .\end{array}$ \\
\hline $\begin{array}{l}\text { Incomplete outcome data } \\
\text { (attrition bias) } \\
\text { All outcomes }\end{array}$ & Low risk & Comment: dropouts were unrelated to the study \\
\hline
\end{tabular}


Kelner 1963 (Continued)

Selective reporting (re- High risk Comment: some aspects of the methodology were not reported (brushing freporting bias) quency, who brushed, dental treatment received during the 4-month trial).

Kissel 1983

Study design: ITS
Date of study: not stated, pre-1983
Study duration: 100 days
Location: USA
Setting: residential
Ethical approval: none reported
Consent: unclear - staff recommended for training and agree to participate

Participants

Description of ID used: "severely and profoundly retarded...(IQ range $=8$ to 21$)$ "

Conversion to ICD description: severe and profound

Age: staff: mean 25 years; residents: range $8-16$ years

Sex: staff: female 4; residents: male 9, female 3 (Figures 2, 4 and 6)

Comorbidity reported/details: none reported

Number of participants at baseline: 4 staff and 12 residents

Number of participants at final evaluation: 4 staff and 12 residents

Selection of the participants for the intervention: staff recommended for training by the supervisors based on their perceived need for training.

Interventions

\section{Comparison: training of carers of people with ID versus no training of carers}

Group 1: staff were trained and given written materials necessary to teach their residents in a 9-step toothbrushing routine using verbal instruction, physical guidance and contingent reinforcement, received feedback on this teaching using a video of the teaching sessions, p. 397.2 intensive training sessions were given to the staff, 1 at the start of the treatment session and 1 at the start of the maintenance sessions.

Group 2: no training

Outcomes

1. Staff scored on use of the 3 levels of skills used when training residents

2. Residents scored on their response at three levels - self-initiated, verbally instructed or physically guided responses

3. Residents scored on the number of steps achieved in the toothbrushing programme

4. Staff Acceptability Questionnaire (Table 3)

Timing of outcome assessments: daily - data presented as mean score during baseline and maintenance phases (100 days)

COM-B System Characteristics
Potential sources of behaviour change: staff: capability (physical/psychological), opportunity (social/physical), motivation (reflective); residents: capability (physical), opportunity (physical), motivation (reflective) 
Kissel 1983 (Continued)

Potential intervention functions: staff: training, modelling, enablement, incentivisation, coercion, restrictions, environmental restructuring; residents: training, modelling, enablement, environmental restructuring

$\begin{array}{ll}\text { Stakeholder Involvement } & \text { Formal or non-formal carer: formal } \\ & \text { With or without dental professional involvement: without } \\ & \begin{array}{l}\text { Other stakeholder involvement: observers ( } 2 \text { administrators, a speech and hearing therapist, a gradu- } \\ \text { ate student intern) }\end{array}\end{array}$

Notes

Strengths and weaknesses: "continual presence of observers could have effected outcomes," "use of videotape feedback greatly aided staff learning," "the training program was found to be very time efficient, with each participant receiving only 3-4 hours of individual instruction from the experimenter during the 6 months of the project," p. 414.

Modifications to the intervention: 1 staff member and resident started the programme later than the others, at session 36 .

Adverse effects: none reported

Funding source: "This research was supported in part by a training grant from the National Institute of Health (1 T32 HDO7184-02)," p. 395.

\section{Risk of bias}

Bias Authors' judgement Support for judgement

Was the intervention inde- High risk pendent of other changes? (ITS)

Comment: training method was being used for 2 other personal skills at the same time.

$\begin{aligned} & \text { Was the shape of the in- } \\ & \text { tervention effect prespeci- } \\ & \text { fied? (ITS) }\end{aligned}$
$\begin{aligned} & \text { Was the intervention un- } \\ & \begin{array}{l}\text { likely to affect data collec- } \\ \text { tion? (ITS) }\end{array}\end{aligned}$

\begin{tabular}{lll}
\hline $\begin{array}{l}\text { Was knowledge of the al- } \\
\text { located interventions ade- }\end{array}$ & High risk & Comment: not possible to blind. \\
quately prevented during & & \\
the study? (ITS) & & \\
\hline $\begin{array}{l}\text { Were incomplete out- } \\
\text { come data adequately ad- } \\
\text { dressed? (ITS) }\end{array}$ & Low risk & Comment: no incomplete data.
\end{tabular}

Was the study free from Unclear risk Comment: no protocol but all listed outcomes reported.
selective outcome report-
ing? (ITS)

\begin{tabular}{l} 
Lange 1985 \\
\hline Methods \\
$\begin{array}{l}\text { Study design: quasi-RCT } \\
\text { Date of study: not stated, pre-1981 }\end{array}$
\end{tabular}




\section{Study duration: 11 weeks}

Location: USA (based on author address)

Setting: residential

Ethical approval: unclear, "Prior to beginning the study the research proposal was reviewed by the administrative staff of L.O.M.R.disseration" thesis, p. 28.

Consent: Yes, "informed consents had to be obtained from subjects and their guardians," "a release of information was signed by the subject and his/her guardian," thesis, p. 28.

\section{Participants}

Description of ID used: mild and moderate mentally retarded. Thesis, p. 30.

Conversion to ICD description: mild (9 participants) and moderate (6 participants)

Age range: $16-39$ years

Sex: male 7 , female 8

Comorbidity reported/details: none reported

Number of participants at baseline: 15

Number of participants at final evaluation: 15

Selection of the participants for the intervention: no details

\section{Interventions Comparison: training of people with ID versus no training of people of ID}

Group 1: carers disclosed and recorded plaque levels, participants trained by carers to remove all disclosed plaque by toothbrushing. Verbal and physical instruction was provided if needed. Praise given as feedback and faded as steps achieved. Maintenance - participants told they would be monitored on a regular basis.

Group 2: carers disclosed and recorded plaque levels after the participant had brushed; no training provided to participants.

Group 3: no regular recording of plaque levels or training.

Outcomes 1 . Plaque Index scored $0-3$

\section{Gingival Index scored $0-3$}

Timing of outcome assessments: plaque - daily, gingival - weekly. Data presented as mean scores collected over the baseline and maintenance periods (5-7 weeks).

\section{COM-B System Character- Potential sources of behaviour change: capability (physical/psychological), motivation (reflective) istics \\ Potential intervention functions: training, modelling, enablement, persuasion, environmental restruc- turing}

\section{Stakeholder Involvement Formal or non-formal carer: formal}

With or without dental professional involvement: without - assessor was trained in the use of the periodontal probe

Other stakeholder involvement: house parents and aides in homes

\section{Notes}

Strengths and weaknesses: self-help skills process was already in use in the facility for other life skills. Group 3 outcomes were only recorded at final time point.

Modifications to the intervention: participants were to record their own plaque scores, but none were able to achieve this skill, so it was recorded by the staff. The baseline period need to be extended due 
to unstable plaque levels. Some participants continued to require cues to complete the toothbrushing task throughout the study.

Adverse effects: none reported

Funding source: none reported

\section{Risk of bias}

\begin{tabular}{|c|c|c|}
\hline Bias & Authors' judgement & Support for judgement \\
\hline \multirow[t]{2}{*}{$\begin{array}{l}\text { Random sequence genera- } \\
\text { tion (selection bias) }\end{array}$} & Unclear risk & $\begin{array}{l}\text { Quote: "Randomised into matching groups by age, sex and functioning abili- } \\
\text { ty." }\end{array}$ \\
\hline & & Comment: no further details provided. \\
\hline $\begin{array}{l}\text { Allocation concealment } \\
\text { (selection bias) }\end{array}$ & Unclear risk & Comment: no details provided. \\
\hline $\begin{array}{l}\text { Blinding of participants } \\
\text { and personnel (perfor- } \\
\text { mance bias) } \\
\text { All outcomes }\end{array}$ & Unclear risk & $\begin{array}{l}\text { Comment: not possible to blind personnel, unclear if participants were blind- } \\
\text { ed. }\end{array}$ \\
\hline $\begin{array}{l}\text { Blinding of outcome as- } \\
\text { sessment (detection bias) } \\
\text { All outcomes }\end{array}$ & High risk & $\begin{array}{l}\text { Quote: "Throughout the study each subject's plaque and gingival indexes were } \\
\text { measured and recorded by the staff and/or the investigator." Thesis, p. } 36 .\end{array}$ \\
\hline $\begin{array}{l}\text { Incomplete outcome data } \\
\text { (attrition bias) } \\
\text { All outcomes }\end{array}$ & Low risk & Comment: no missing data. \\
\hline $\begin{array}{l}\text { Selective reporting (re- } \\
\text { porting bias) }\end{array}$ & Unclear risk & $\begin{array}{l}\text { Comment: no protocol but all listed outcomes reported. Group } 3 \text { had only } 1 \\
\text { time point measured (final). }\end{array}$ \\
\hline
\end{tabular}

Lange 2000

$\begin{array}{ll}\text { Methods } & \text { Study design: NRCT } \\ \text { Date of study: not stated, pre-2000 } & \text { Study duration: } 21 \text { days } \\ \text { Location: USA } & \text { Setting: residential } \\ \text { Ethical approval: none reported } \\ \text { Consent: "After explaining the study to the parents and/or legal guardians of the clients, the re- } \\ \text { searchers provided the institution administration with a signed informed consent for each client in- } \\ \text { cluded in the study," p. 206. } \\ \text { Description of ID used: "moderately to profoundly mentally retarded" p. 207 } \\ \text { Conversion to ICD description: moderate to profound } \\ \text { Age (mean): intervention group: } 43 \text { years (SD 12), control group: } 42 \text { years (SD 12) } \\ \text { Sex: male 42, female 34 }\end{array}$


Comorbidity reported/details: none reported

Number of participants at baseline: 64

Number of participants at final evaluation: paired data 60/61

Selection of the participants for the intervention: selected by unit directors

Interventions Comparison: training of carers versus no training of carers

New policy for toothbrushing twice a day introduced, old policy indicated 4 times a day

Group 1: training with accountability - care staff were trained by dental professionals to help clients brush, to brush for the non-compliant clients and disclose and chart the plaque levels and informed about new policy. Feedback was given and copies given to the unit leaders.

Group 2: training without accountability - care staff were trained by staff development personnel on how to brush and monitor their own oral hygiene and informed about new policy. They were instructed to put this training into use with their clients and trained to monitor client progress

Group 3: no training, so not informed about change to toothbrushing policy

We combined data from groups 1 and 2 .

Outcomes

1. Plaque Index - Modified Ramfjord Periodontal Disease Index - plaque only, on 6 standard teeth, with disclosing solution, p. 206

Timing of outcome assessments: Group 1 and Group 2: baseline, 7, 14 and 21 days. Group 3: baseline and 21 days

COM-B System Characteristics

Potential sources of behaviour change: capability (physical/psychological), opportunity (physical), motivation (reflective)

Potential intervention functions: education, training, modelling, enablement, incentivisation, coercion, environmental restructuring

\section{Stakeholder Involvement $\quad$ Formal or non-formal carer: formal}

With or without dental professional involvement: with - dental assistant, dental hygienist

Other stakeholder involvement: administration, living unit directors, staff development department, registered general nurse, dental department staff, carers

Notes Strengths and weaknesses: short duration, potential additional expenses - additional staff to provide monitoring/feedback.

Modifications to the intervention: none reported

Adverse effects: none reported

Funding source: none reported

\section{Risk of bias}

\begin{tabular}{lll}
\hline Bias & Authors' judgement & Support for judgement \\
\hline $\begin{array}{l}\text { Random sequence genera- } \\
\text { tion (selection bias) }\end{array}$ & High risk & $\begin{array}{l}\text { Comment: authors acknowledged that randomisation was not possible, p. } \\
207 .\end{array}$ \\
\hline $\begin{array}{l}\text { Allocation concealment } \\
\text { (selection bias) }\end{array}$ & Low risk & $\begin{array}{l}\text { Quote: "The unit director drew numbers to determine if their unit would be the } \\
\text { control, training with accountability (experimental group 1) or training with- } \\
\text { out accountability (experimental group II). They were not told the status of } \\
\text { their unit," p. 206. }\end{array}$ \\
\hline
\end{tabular}


Comment: assumed to be done correctly.

\begin{tabular}{|c|c|c|}
\hline $\begin{array}{l}\text { Blinding of participants } \\
\text { and personnel (perfor- } \\
\text { mance bias) } \\
\text { All outcomes }\end{array}$ & Low risk & $\begin{array}{l}\text { Quote: "Living unit staff, other than through staff development, had no oppor- } \\
\text { tunity to interact with staff from other living units," p. } 208 .\end{array}$ \\
\hline
\end{tabular}

\begin{tabular}{|c|c|c|}
\hline $\begin{array}{l}\text { Blinding of outcome as- } \\
\text { sessment (detection bias) } \\
\text { All outcomes }\end{array}$ & Low risk & $\begin{array}{l}\text { Quote: "The examiner did not know the client's group status at the time of the } \\
\text { plaque assessment," p. } 206 .\end{array}$ \\
\hline
\end{tabular}

Incomplete outcome data Low risk Comment: missing data clearly explained; unlikely to affect outcomes.
(attrition bias)

(attrition bias)

Selective reporting (re- Unclear risk Comment: no protocol but all listed outcomes reported.

porting bias)

\section{Mac Giolla Phadraig 2015}

Study design: cluster RCT - the unit of randomisation was the residential home (Mac Giolla Phadraig
2015, p. 93).
Date of study: July 2008 to September 2009
Study duration: 6-11 months
Location: Ireland
Setting: residential
Ethical approval: "Ethical approval for the study was received from the relevant research ethics bod-
ies," p. 93 (2015).
Consent: yes - "consent and assent" figure 2, p. 94 (2015) "consent forms were sent to all care staff in
the test organization two to 6 weeks before training was delivered to the intervention group," p. 185
(MacGiolla Phadraig 2013).

Participants

Description of ID used: "carer-reported severity of ID (mild to profound)"

Conversion to ICD description: mixed (mild to profound)

Age (mean): people with ID - intervention group: 43 years (SD 12); control group: 42 years (SD 12)

Sex: carers: not reported; people with ID: 42 male 34, female, p. 95 (2015)

Comorbidity reported/details: none reported

Number of participants at baseline: people with ID 64, carers 187. Number of residential units: 50

Number of participants at final evaluation: people with ID - paired data 60/61, carers 154

Selection of the participants for the intervention: randomly selected from a large ID service provider

\section{Interventions Comparison: training of carers versus no training of carers}

Group 1: a day-long education and training session provided by oral health trainers, with practical sessions and a specifically designed training pack to train their peers with motivational discussion, a moral agreement with dental team and offer of ongoing support if needed. 
Mac Giolla Phadraig 2015 (Continued)

$$
\text { Group 2: no training }
$$

1. Knowledge, behaviour, attitude and self-efficacy of carers via a questionnaire
2. Modified Gingival Index
3. Modified Silness \& Löe Plaque Index - "no probe used / only surfaces visible to the researcher," p. 94
(2015)
Timing of outcome assessments: baseline and 10 months

COM-B System Character- Potential sources of behaviour change: capability (physical/psychological), opportunity (physical), moistics tivation (reflective)

Potential Intervention functions: education, training, modelling, enablement, environmental restructuring

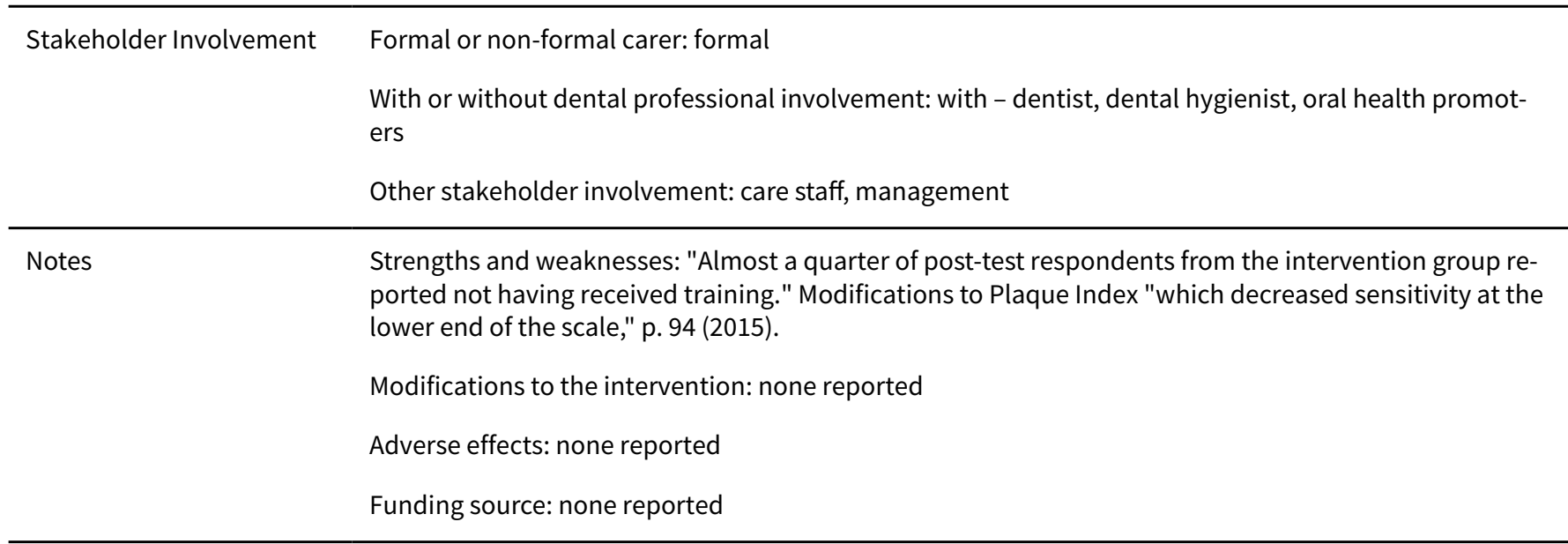

\section{Risk of bias}

\begin{tabular}{|c|c|c|}
\hline Bias & Authors' judgement & Support for judgement \\
\hline \multirow[t]{2}{*}{$\begin{array}{l}\text { Random sequence genera- } \\
\text { tion (selection bias) }\end{array}$} & Low risk & $\begin{array}{l}\text { Quote: "Each group was then randomly allocated as either control or interven- } \\
\text { tion," p. } 184 \text { (2013). }\end{array}$ \\
\hline & & Comment: probably done. \\
\hline $\begin{array}{l}\text { Allocation concealment } \\
\text { (selection bias) }\end{array}$ & Low risk & Comment: no details of randomisation method but probably done. \\
\hline $\begin{array}{l}\text { Blinding of participants } \\
\text { and personnel (perfor- } \\
\text { mance bias) } \\
\text { All outcomes }\end{array}$ & Unclear risk & Comment: no details provided in relation to blinding of personnel. \\
\hline \multirow{2}{*}{$\begin{array}{l}\text { Blinding of outcome as- } \\
\text { sessment (detection bias) } \\
\text { All outcomes }\end{array}$} & Unclear risk & $\begin{array}{l}\text { Quote: "One experienced dentist carried out all examinations following cali- } \\
\text { bration exercises," p. 94. (2015). }\end{array}$ \\
\hline & & Comment: questionnaires were anonymous but no details of key holder. \\
\hline $\begin{array}{l}\text { Incomplete outcome data } \\
\text { (attrition bias) } \\
\text { All outcomes }\end{array}$ & High risk & $\begin{array}{l}\text { Comment: attrition rate of carers was high; no clear reason for dropouts identi- } \\
\text { fied. }\end{array}$ \\
\hline
\end{tabular}


Mac Giolla Phadraig 2015 (Continued)

Selective reporting (re- Unclear risk Comment: no protocol but all listed outcomes reported. porting bias)

Olmos 2016

$\begin{array}{ll}\text { Methods } & \text { Study design: RCT } \\ \text { Date of study: September } 2007 \text { to February } 2008 \\ \text { Study duration: } 4 \text { weeks } \\ \text { Location: Germany } \\ \text { Setting: residential } \\ \text { Ethical approval: not reported } \\ \text { Consent: not reported }\end{array}$

Participants

Description of ID used: "special needs"

Conversion to ICD description: unclear

Age range (mean): $28-74$ years (46.5)

Sex: male 98, female 95

Comorbidity reported/details: none reported

Number of participants at baseline: 193

Number of participants at final evaluation: 193

Selection of the participants for the intervention: invited to attend from a list of persons with disabilities in a city.

Interventions

\section{Comparison: training of carers versus no training of carers}

Group 1: carer and participant were provided with theoretical and practical training on oral hygiene.

Group 2: usual care

Outcomes

1. Type of toothbrush

2. Type of toothpaste

3. Frequency of fluoride application

4. Timing of toothbrushing

5. Duration of toothbrushing

Timing of outcome assessments: baseline, week 4

COM-B System Characteristics
Potential sources of behaviour change: capability (physical/psychological), opportunity (physical), motivation (reflective)

Potential intervention functions: education, training, motivation, enablement, environmental restructuring

Formal or non-formal carer: formal 
Other stakeholder involvement: none reported

\section{Notes}

Strengths and weaknesses: short duration, followed up for 5 years, but the control group received the intervention immediately after this study so no long-term control.

Modifications to the intervention: none reported

Adverse effects: none reported

Funding source: yes. "The project has been carried out since 2005 by the non-profit Berliner Hilfswerk Zahnmedizin e.V. with the support of the Senate Department for Health and Social Affairs," (Olmos 2017).

\section{Risk of bias}

\begin{tabular}{|c|c|c|}
\hline Bias & Authors' judgement & Support for judgement \\
\hline \multirow[t]{2}{*}{$\begin{array}{l}\text { Random sequence genera- } \\
\text { tion (selection bias) }\end{array}$} & Unclear risk & $\begin{array}{l}\text { Quote: "randomised assignment of the participants to a control and an inter- } \\
\text { vention group." }\end{array}$ \\
\hline & & Comment: no details provided \\
\hline $\begin{array}{l}\text { Allocation concealment } \\
\text { (selection bias) }\end{array}$ & Unclear risk & Comment: randomly assigned but no details provided. \\
\hline $\begin{array}{l}\text { Blinding of participants } \\
\text { and personnel (perfor- } \\
\text { mance bias) } \\
\text { All outcomes }\end{array}$ & Unclear risk & Comment: no details provided. \\
\hline $\begin{array}{l}\text { Blinding of outcome as- } \\
\text { sessment (detection bias) } \\
\text { All outcomes }\end{array}$ & Unclear risk & Comment: no details provided. \\
\hline $\begin{array}{l}\text { Incomplete outcome data } \\
\text { (attrition bias) } \\
\text { All outcomes }\end{array}$ & Low risk & Comment: no missing data. \\
\hline $\begin{array}{l}\text { Selective reporting (re- } \\
\text { porting bias) }\end{array}$ & Unclear risk & Comment: no protocol but all listed outcomes reported. \\
\hline
\end{tabular}

\section{Phlypo 2016}

Study design: RCT
Date of study: not stated, pre-2016
Study duration: 8 weeks
Location: Belgium
Setting: residential
Ethical approval: yes
Consent: yes - "For every selected resident, the parents or guardian granted their permission to partici-
pate," p. 5.


Phlypo 2016 (Continued)
Participants
Description of ID used: severe to profound ID
Conversion to ICD description: severe, profound
Age range (mean): not reported
Sex: not reported
Comorbidity reported/details: not reported
Number of participants at baseline: 55 carers
Number of participants at final evaluation: 34 carers
Selection of the participants for the intervention: not reported

\section{Interventions Comparison: training of carers versus no training of carers}

Group 1: carers provided with an information booklet and 1 information session with practical skills Group 2: usual care
Outcomes
1. Knowledge, behaviour, attitude and self-efficacy of carers
2. Plaque Index of people with ID
3. Gingival Index of people with ID
Timing of outcome assessments: 3 weeks before the intervention, 5 weeks' postintervention

COM-B System Character- Potential sources of behaviour change: capability (physical/psychological), opportunity (physical), moistics tivation (reflective)

Potential intervention functions: education, training, enablement, environmental restructuring

$\begin{array}{ll}\text { Stakeholder Involvement } & \text { Formal or non-formal carer: formal } \\ & \text { With or without dental professional involvement: with - dental students } \\ & \text { Other stakeholder involvement: none reported }\end{array}$

Notes

Strengths and weaknesses: high attrition rate; short duration. A panel discussion with the resident dentists and director at baseline may have influenced the control group.

Modifications to the intervention: none reported

Adverse effects: none reported

Funding source: none reported

\section{Risk of bias}

\begin{tabular}{lll}
\hline Bias & Authors' judgement & Support for judgement \\
\hline $\begin{array}{l}\text { Random sequence genera- } \\
\text { tion (selection bias) }\end{array}$ & Unclear risk & Quote: "Randomly selected to be clinically examined," p. 4. \\
& & Comment: no details of process. \\
\hline $\begin{array}{l}\text { Allocation concealment } \\
\text { (selection bias) }\end{array}$ & Unclear risk & Comment: no details provided. \\
\hline
\end{tabular}


Phlypo 2016 (Continued)

Blinding of participants Unclear risk Comment: no details provided. and personnel (performance bias)

All outcomes

\begin{tabular}{lll}
\hline $\begin{array}{l}\text { Blinding of outcome as- } \\
\text { sessment (detection bias) } \\
\text { All outcomes }\end{array}$ & Unclear risk & Comment: no details provided. \\
\hline $\begin{array}{l}\text { Incomplete outcome data } \\
\begin{array}{l}\text { (attrition bias) } \\
\text { All outcomes }\end{array}\end{array}$ & High risk & $\begin{array}{l}\text { Comment: high attrition rate. Unclear why there were such high numbers who } \\
\text { failed to complete assessment questionnaire or read the booklet. }\end{array}$ \\
\hline $\begin{array}{l}\text { Selective reporting (re- } \\
\text { porting bias) }\end{array}$ & Unclear risk & Comment: no protocol, but all listed outcomes reported. \\
\hline
\end{tabular}

\section{Sauvetre 1995}

\section{Methods}

Study design: RCT

Date of study: not stated, pre-1995

Study duration: 21 days

Location: Belgium

Setting: day centre

Ethical approval: "The study got the permission of the "Helsinki Commitions [Commissions] of the Brugmann University Hospital," "The authors respected all though the study the "Helsinki declaration" - (1989)," p. 116.

Consent: none reported, but assumed as per Helsinki declaration.

\section{Participants}

Description of ID used: "mental retardation," p. 116

Conversion to ICD description: unclear

Age range (mean): $18-40$ years

Sex: not reported

Comorbidity reported/details: none reported

Number of participants at baseline: 25

Number of participants at final evaluation: 25

Selection of the participants for the intervention: randomly chosen from a day centre.

Interventions

\section{Comparison: special manual toothbrush versus conventional manual toothbrush}

All participants received instructions on how to brush "using the simplest method (horizontal movements with short strokes)." Asked to brush twice a day for at least 60 seconds. Reinstructed on day 7 .

Group 1: Superbrush, 3-headed toothbrush

Group 2: Oral B P35, standard toothbrush 
2. Bleeding Index (Saxer 1975) for 6 standardised teeth (Ramfjord 1959). From 3 sites - buccal, mesial and lingual, p. 116

Timing of outcome assessments: baseline, day 7 and day 21

COM-B System Character- Potential sources of behaviour change: capability (physical), opportunity (physical)

istics

Potential intervention functions: training, environmental restructuring

Stakeholder Involvement Formal or non-formal carer: not reported

With or without dental professional involvement: with - periodontist, dental hygienist

Other stakeholder involvement: none reported

Notes

Strengths and weaknesses: no mention if monitoring of toothbrushing took place; very short study

Modifications to the intervention: 5 eliminated as unable to follow training to brush their teeth as was required, p. 116.

Adverse effects: none reported

Funding source: "This work was supported by DENTACO S.A. Norway," who produced the Superbrush.

\section{Risk of bias}

\begin{tabular}{|c|c|c|}
\hline Bias & Authors' judgement & Support for judgement \\
\hline $\begin{array}{l}\text { Random sequence genera- } \\
\text { tion (selection bias) }\end{array}$ & Low risk & Comment: reports randomly selected, double blind; probably done. \\
\hline $\begin{array}{l}\text { Allocation concealment } \\
\text { (selection bias) }\end{array}$ & Low risk & $\begin{array}{l}\text { Comment: reports that the brushes were distributed by an oral hygienist im- } \\
\text { mediately after the baseline assessment, who was not the assessor, p. } 116 . \\
\text { "Double blind trial" - probably done. }\end{array}$ \\
\hline $\begin{array}{l}\text { Blinding of participants } \\
\text { and personnel (perfor- } \\
\text { mance bias) } \\
\text { All outcomes }\end{array}$ & Low risk & $\begin{array}{l}\text { Comment: control group were also asked to do something that was not usual } \\
\text { care. }\end{array}$ \\
\hline $\begin{array}{l}\text { Blinding of outcome as- } \\
\text { sessment (detection bias) } \\
\text { All outcomes }\end{array}$ & Low risk & $\begin{array}{l}\text { Quote: "double-blind trial" } \\
\text { Comment: probably done. }\end{array}$ \\
\hline $\begin{array}{l}\text { Incomplete outcome data } \\
\text { (attrition bias) } \\
\text { All outcomes }\end{array}$ & Unclear risk & $\begin{array}{l}\text { Comment: } 5 \text { "intention to treat" participants were excluded as they were un- } \\
\text { able to follow the training. }\end{array}$ \\
\hline $\begin{array}{l}\text { Selective reporting (re- } \\
\text { porting bias) }\end{array}$ & Unclear risk & Comment: no protocol but all listed outcomes reported. \\
\hline
\end{tabular}

\section{Shaw 1983}

Methods

Study design: RCT, cross-over trial

Date of study: not stated, pre-1983

Study duration: 4 weeks for each arm of the trial 
Shaw 1983 (Continued)

Location: UK

Setting: school

Ethical approval: not reported

Consent: parents gave consent for the project, p. 5 .

Description of ID used: severely mentally retarded, p. 4
Conversion to ICD description: severe
Age range: $9-16$ years
Sex: not reported
Comorbidity reported/details: "a range of handicapping conditions," p. 4
Number of participants at baseline: 66
Number of participants at final evaluation: 53
Selection of the participants for the intervention: the schools agreed to participate; children selected
by the staff.

Interventions

\section{Comparison: electric toothbrush versus manual toothbrush}

Individual $\mathrm{OHI}$ with a disclosing solution using the designated toothbrush. No specific technique was taught, but instructed to "get the stain off" and encouraged to adopt a systematic approach. All children brushed their own teeth, but with initial guidance and assistance. Brushing was carried out in the classroom for 5 minutes every day.

Group 1: Braun Electric rechargeable electric toothbrush

Group 2: Oral B 30 manual toothbrush

1. Plaque Index - Silness \& Löe (V)
2. Bleeding Index (WHO Technical Report No 621, 1971) on 6 selected teeth (V)
$\begin{aligned} & \text { Timing of outcome assessments: baseline, } 4 \text { weeks, (washout period during "a prolonged school holi- } \\ & \text { day"), } 4 \text { weeks later }\end{aligned}$

COM-B System Character- Potential sources of behaviour change: capability (physical /psychological), opportunity (social/physiistics cal), motivation (reflective)

Potential intervention functions: training, enablement, persuasion, incentivisation, environmental restructuring

Stakeholder Involvement Formal or non-formal carer: formal

With or without dental professional involvement: with - dentist and dental therapist

Other stakeholder involvement: teachers

Notes

Strengths and weaknesses: "The teaching staff saw the project as one of socialisation and the development of self-care skills which they regarded as important. Class charts which were filled in with stars when the children had completed their brushing for the day served as a useful reminder for the staff and as motivation for the children," p. 5.

Modifications to the intervention: none reported

Adverse effects: none reported 
Funding source: "We are grateful to Braun Electric (UK) Ltd for the provision of rechargeable electric toothbrushes and to Cooper Health Products for the Oral B 30 toothbrushes," p. 5.

\section{Risk of bias}

\begin{tabular}{lll}
\hline Bias & Authors' judgement & Support for judgement \\
\hline $\begin{array}{ll}\text { Random sequence genera- } \\
\text { tion (selection bias) }\end{array}$ & Low risk & $\begin{array}{l}\text { Quote: "The school staff randomly selected } 66 \text { children, randomly allocated to } \\
\text { test or control," p. 4. } \\
\text { Comment: probably done. }\end{array}$ \\
\hline $\begin{array}{ll}\text { Allocation concealment } \\
\text { (selection bias) }\end{array}$ & Low risk & $\begin{array}{l}\text { Quote: "The school staff randomly selected } 66 \text { children, randomly allocated to } \\
\text { test or control," p. 4. }\end{array}$ \\
& Comment: probably done. \\
\hline
\end{tabular}

Blinding of participants High risk Comment: not possible to blind participants or personnel.
and personnel (perfor-

mance bias)

All outcomes

\begin{tabular}{lll}
\hline $\begin{array}{l}\text { Blinding of outcome as- } \\
\text { sessment (detection bias) } \\
\text { All outcomes }\end{array}$ & Low risk & $\begin{array}{l}\text { Quote: "At no time was the examiner aware of the group to which the children } \\
\text { had been assigned," p. } 4 .\end{array}$ \\
\hline $\begin{array}{l}\text { Incomplete outcome data } \\
\text { (attrition bias) } \\
\text { All outcomes }\end{array}$ & High risk & Comment: 20\% attrition unexplained \\
\hline $\begin{array}{l}\text { Selective reporting (re- } \\
\text { porting bias) }\end{array}$ & Unclear risk & $\begin{array}{l}\text { Comment: unclear if results are reported for the combined arms. Some mid- } \\
\text { way results provided in text. }\end{array}$ \\
\hline
\end{tabular}

Shaw 1991

$\begin{array}{ll}\text { Methods } & \text { Study design: cluster RCT } \\ \text { Date of study: } 1986-1988 \\ \text { Study duration: } 2 \text { years } \\ \text { Location: UK } \\ \text { Setting: day centre } \\ \text { Ethical approval: none reported } \\ \text { Consent: yes, p. } 140 \text { (Shaw 1991) } \\ \text { Description of ID used: "with mental handicaps," pp. 139-40 } \\ \text { Conversion to ICD description: unclear } \\ \text { Age (mean): } 30.9 \text { years (SD 10.1) } \\ \text { Sex: males 168, females } 161 \text { (Shaw 1990, p. 137) } \\ \text { Comorbidity reported/details: yes, "54.1\% had a comorbidity reported," "Physical handicap, epilepsy, } \\ \text { Down syndrome, speech difficulties and miscellaneous" (Shaw 1990, p. 136) }\end{array}$


Shaw 1991 (Continued)

Number of participants at baseline: 329

Number of participants at final evaluation: 304

Selection of the participants for the intervention: 4 largest training centres for adults in a UK city approached.

Interventions

\section{Comparison: daily supervised toothbrushing and scheduled dental recall visits at 1-, 3- or 6- month intervals versus usual care}

Group 1: no specific treatment

Group 2: daily toothbrushing supervised by staff in the centre and reinforced OHI every 6 months

Group 3: daily toothbrushing supervised by staff with prophylaxis and reinforced $\mathrm{OHI}$ every month

Group 4: daily toothbrushing supervised by staff with prophylaxis and reinforced $\mathrm{OHI}$ every 3 months

\begin{tabular}{|c|c|c|}
\hline \multirow[t]{7}{*}{ Outcomes } & \multicolumn{2}{|c|}{ 1. DMFT - only reported at baseline } \\
\hline & \multicolumn{2}{|c|}{ WHO Technical Report using 6 standard teeth for } \\
\hline & \multicolumn{2}{|l|}{ 2. Plaque } \\
\hline & \multicolumn{2}{|l|}{ 3. Calculus } \\
\hline & \multicolumn{2}{|c|}{ 4. Periodontal pocketing } \\
\hline & \multicolumn{2}{|l|}{ 5. Bleeding } \\
\hline & \multicolumn{2}{|c|}{ Timing of outcome assessments: baseline, $3,6,12,18$ and 24 months } \\
\hline \multirow[t]{2}{*}{$\begin{array}{l}\text { COM-B System Character- } \\
\text { istics }\end{array}$} & \multirow{2}{*}{\multicolumn{2}{|c|}{$\begin{array}{l}\text { Potential sources of behaviour change: carers and people with ID: capability (physical/psychological), } \\
\text { opportunity (physical), motivation (reflective) } \\
\text { Potential intervention functions: carers: environmental restructuring. People with ID: training, enable- } \\
\text { ment, persuasion, environmental restructuring }\end{array}$}} \\
\hline & & \\
\hline \multirow[t]{3}{*}{ Stakeholder Involvement } & \multicolumn{2}{|c|}{ Formal or non-formal carer: formal } \\
\hline & \multicolumn{2}{|c|}{ With or without dental professional involvement: with - dental hygienist } \\
\hline & \multicolumn{2}{|c|}{ Other stakeholder involvement: staff supervising toothbrushing } \\
\hline \multirow[t]{4}{*}{ Notes } & \multicolumn{2}{|c|}{$\begin{array}{l}\text { Strengths and weaknesses: the difference in time involvement was very significant when cost assess- } \\
\text { ments were made long-term, with applied models of professional support }\end{array}$} \\
\hline & \multicolumn{2}{|c|}{ Modifications to the intervention: none reported } \\
\hline & \multicolumn{2}{|c|}{ Adverse effects: none reported } \\
\hline & \multicolumn{2}{|c|}{ Funding source: supported by a grant from the West Midlands Regional Health Authority, p. 140 (1990) } \\
\hline \multicolumn{3}{|l|}{ Risk of bias } \\
\hline Bias & Authors' judgement & Support for judgement \\
\hline $\begin{array}{l}\text { Random sequence genera- } \\
\text { tion (selection bias) }\end{array}$ & Unclear risk & $\begin{array}{l}\text { Quote: "Each of the centres was then randomly allocated to one of four treat- } \\
\text { ment regimens," p. } 140 .\end{array}$ \\
\hline & & Comment: no further details provided. \\
\hline
\end{tabular}


Shaw 1991 (Continued)

Allocation concealment $\quad$ Unclear risk $\quad$ Comment: no details provided.
(selection bias)

$\begin{array}{ll}\begin{array}{l}\text { Blinding of participants } \\ \text { and personnel (perfor- }\end{array} & \text { Low risk } \\ \text { Comment: unlikely that each centre would have been aware of what the other } \\ \text { centres were doing }\end{array}$
mance bias)

All outcomes

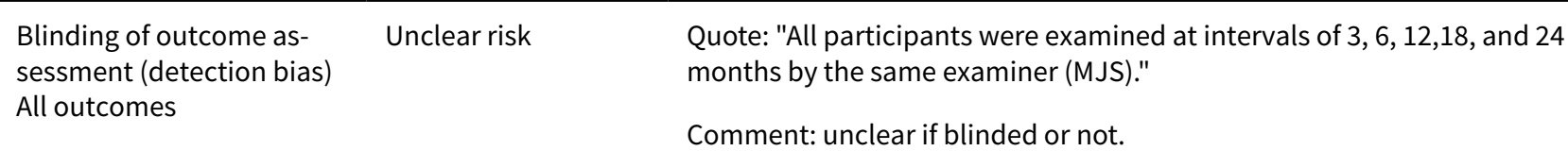

$\begin{array}{ll}\begin{array}{l}\text { Incomplete outcome data } \\ \text { (attrition bias) }\end{array} & \text { Low risk } \\ \text { All outcomes } & \begin{array}{l}\text { Comment: details of numbers of participants only provided at baseline and fi- } \\ \text { nal measure (Table 1, p. 141), but clear reasons given for dropouts. Dropouts } \\ \text { occurred in all groups and were unlikely to affect outcomes. }\end{array}\end{array}$

Selective reporting (re- Unclear risk $\quad$ Comment: no protocol but all listed outcomes reported.
porting bias)
porting bias)

\section{Snell 1989}

\begin{tabular}{|c|c|}
\hline \multirow[t]{7}{*}{ Methods } & Study design: repeat measure \\
\hline & Date of study: not stated, pre-1989 \\
\hline & Study duration: 3 years \\
\hline & Location: USA \\
\hline & Setting: school \\
\hline & Ethical approval: none reported \\
\hline & Consent: none reported \\
\hline \multirow[t]{8}{*}{ Participants } & Description of ID used: "severe mental retardation " and "profound mental retardation" \\
\hline & Conversion to ICD description: severe ( 2 participants) and profound (1 participant) \\
\hline & Age range: $5-11$ years \\
\hline & Sex: males 2 , females 1 \\
\hline & $\begin{array}{l}\text { Comorbidity reported/details: yes, severe quadriplegic, athetoid cerebral palsy, spastic cerebral palsy, } \\
\text { visual impairment }\end{array}$ \\
\hline & Number of participants at baseline: 3 \\
\hline & Number of participants at final evaluation: 2 (1 lost at 19-month follow-up) \\
\hline & Selection of the participants for the intervention: no details provided \\
\hline
\end{tabular}

\section{Comparison: training of people with ID}

Group 1: training in 3 tasks - toothbrushing (12 steps), rinsing ( 3 steps) and wiping mouth ( 2 steps) with verbal and physical prompting, which were gradually faded as the training continued, 30 minutes each school day. Praise and tokens used as motivators, which were faded over time. Maintenance started 
Snell 1989 (Continued)

once the skills were completed without prompts for 3-5 days. In this phase, praise was only given on completion of the entire task. Training was reinstated if needed.

1. Number of steps achieved for each task
2. Number of training sessions it took for the skill to be performed without prompting
3. Parents' awareness of changes in oral hygiene routine
4. Teachers' survey, 3 years' postintervention, "questioned on students skill retention," p. 221.
Timing of outcome assessments: baseline, training (unclear, about 120 days), 4-7 months post, 19
months post, 3 years post

COM-B System Characteristics

Potential sources of behaviour change: capability (physical), opportunity (physical), motivation (reflective)

Potential intervention functions: training, enablement, persuasion, incentivisation, environmental restructuring

\section{Stakeholder Involvement $\quad$ Formal or non-formal carer: formal}

With or without dental professional involvement: without

Other stakeholder involvement: teachers, speech therapist and occupational therapist, pp. 21-8.

\section{Notes}

Strengths and weaknesses: small study; inconsistent measures at baseline; modifications to the training, provision of booster training during maintenance phase

Modifications to the intervention: yes, as above

Adverse effects: yes - reported informally, reference to 2 participants displaying sensitivity to mouths being held open, p. 220.

Funding source: none reported

\section{Risk of bias}

\begin{tabular}{lll}
\hline Bias & Authors' judgement & Support for judgement \\
\hline $\begin{array}{l}\text { Was the intervention inde- } \\
\text { pendent of other changes? } \\
\text { (ITS) }\end{array}$ & Unclear risk & Comment: this type of training was used for other skills in the school. \\
\hline $\begin{array}{l}\text { Was the shape of the in- } \\
\text { tervention effect prespeci- } \\
\text { fied? (ITS) }\end{array}$ & Low risk & Comment: the point of analysis was the point of intervention. \\
\hline $\begin{array}{l}\text { Was the intervention un- } \\
\text { likely to affect data collec- } \\
\text { tion? (ITS) }\end{array}$ & Low risk & Comment: the intervention did not affect either the source or method of data \\
\hline $\begin{array}{l}\text { Was knowledge of the al- } \\
\text { located interventions ade- } \\
\text { quately prevented during } \\
\text { the study? (ITS) }\end{array}$ & High risk & Collection. \\
\hline $\begin{array}{l}\text { Were incomplete out- } \\
\text { come data adequately ad- } \\
\text { dressed? (ITS) }\end{array}$ & Low risk & \\
\hline
\end{tabular}


Snell 1989 (Continued)

Was the study free from selective outcome reporting? (ITS)

\section{Swallow 1969}

$\begin{array}{ll}\text { Methods } & \text { Study design: RCT } \\ & \text { Date of study: not stated, pre-1969 } \\ & \text { Study duration: } 21 \text { days } \\ & \text { Location: UK } \\ \text { Setting: residential } \\ \text { Ethical approval: none reported } \\ \text { Consent: none reported }\end{array}$

Participants

Description of ID used: "mentally subnormal," p. 376, "mentally retarded," p. 376. "varying mental ability," p. 377

Conversion to ICD description: mixed

Age range: $15-30$ years

Sex: 40 male, 40 female

Comorbidity reported/details: none reported

Number of participants at baseline: 80

Number of participants at final evaluation: 80

Selection of the participants for the intervention: total sample of patients in the setting examined in "an unbiased order" until 40 males and 40 females meeting the inclusion criteria were found, p. 377.

\section{Comparison 1: electric toothbrush versus manual toothbrush \\ Comparison 2: frequency of toothbrushing}

"Teeth were brushed in their own wards after breakfast or after the midday meal, brushed on alternate occasions by one of the two operators, in order to avoid bias," p. 377 . Males and females were in 2 separate subgroups.

Group 1: teeth brushed once a day with an "automatic toothbrush" (supplied by Ronson Products Ltd)

Group 2: teeth brushed once a day with a manual toothbrush (Colgate-Palmolive)

Group 3: teeth brushed twice a week with an "automatic toothbrush" (supplied by Ronson Products Ltd)

Group 4: teeth brushed twice a week with a manual toothbrush (Colgate-Palmolive)

Group 5: teeth brushed once a week with an "automatic toothbrush" (supplied by Ronson Products Ltd)

Group 6: teeth brushed once a week with a manual toothbrush (Colgate-Palmolive)

Group 7: usual care - "rarely received any regular form of oral hygiene." 
Swallow 1969 (Continued)

We used data from Group 1 and 2 for Comparison 1. We combined data from electric and manual toothbrushes for each of the 3 frequency groups used for Comparison 2.

\begin{tabular}{|c|c|}
\hline \multirow[t]{3}{*}{ Outcomes } & $\begin{array}{l}\text { 1. Gingival Index (Silness \& Löe) - "only the interdental papillae and the intervening gingiva on the buc- } \\
\text { cal surfaces of all standing teeth were scored," p. } 377 \text {. }\end{array}$ \\
\hline & 2. Time spent brushing teeth \\
\hline & Timing of outcome assessments: GI - baseline, day 21; brushing time - day 10 \\
\hline \multirow{2}{*}{$\begin{array}{l}\text { COM-B System Character- } \\
\text { istics }\end{array}$} & Potential sources of behaviour change: opportunity (physical) \\
\hline & Potential intervention functions: environmental restructuring \\
\hline \multirow[t]{3}{*}{ Stakeholder Involvement } & Formal or non-formal carer: formal \\
\hline & With or without dental professional involvement: with - final year dental students, dentist \\
\hline & Other stakeholder involvement: none reported \\
\hline \multirow[t]{4}{*}{ Notes } & Strengths and weaknesses: short intervention \\
\hline & Modifications to the intervention: none reported \\
\hline & Adverse effects: none reported \\
\hline & $\begin{array}{l}\text { Funding source: "Toothbrushes and toothpaste kindly supplied by Colgate-Palmolive. Toothbrushes } \\
\text { kindly supplied by Ronson Products Ltd," p. } 377 \text { footnote. }\end{array}$ \\
\hline
\end{tabular}

\section{Risk of bias}

\begin{tabular}{|c|c|c|}
\hline Bias & Authors' judgement & Support for judgement \\
\hline $\begin{array}{l}\text { Random sequence genera- } \\
\text { tion (selection bias) }\end{array}$ & Low risk & $\begin{array}{l}\text { Quote: "randomly allocated into } 8 \text { groups comprising } 5 \text { males and } 5 \text { females." } \\
\text { Comment: probably done. }\end{array}$ \\
\hline $\begin{array}{l}\text { Allocation concealment } \\
\text { (selection bias) }\end{array}$ & Low risk & Comment: probably done. \\
\hline $\begin{array}{l}\text { Blinding of participants } \\
\text { and personnel (perfor- } \\
\text { mance bias) } \\
\text { All outcomes }\end{array}$ & Unclear risk & $\begin{array}{l}\text { Comment: not possible to blind personnel, brushing was carried out by dental } \\
\text { students; participants may have been blinded as brushing was carried out in } \\
\text { their own bathrooms/units. }\end{array}$ \\
\hline $\begin{array}{l}\text { Blinding of outcome as- } \\
\text { sessment (detection bias) } \\
\text { All outcomes }\end{array}$ & Low risk & $\begin{array}{l}\text { Quote: "Subjects were again examined by the same independent observer } \\
\text { (JNS) who had no knowledge of the grouping of the patients," p. } 377 \text {. }\end{array}$ \\
\hline $\begin{array}{l}\text { Incomplete outcome data } \\
\text { (attrition bias) } \\
\text { All outcomes }\end{array}$ & Low risk & Comment: all participants completed the study, p. 377 . \\
\hline $\begin{array}{l}\text { Selective reporting (re- } \\
\text { porting bias) }\end{array}$ & Unclear risk & Comment: no protocol but all listed outcomes reported. \\
\hline
\end{tabular}

\section{Teitelbaum 2009}

Methods Study design: NRCT - blind cross-over trial - only 2 of the 4 groups are relevant to this review.


Teitelbaum 2009 (Continued)

Date of study: not stated, pre-2009

Study duration: 85 days of which 45 days were washout periods

Location: Brazil

Setting: residential

Ethical approval: "This study was approved by the Joint Research and Ethics Committee of the Ponta Grossa State University Protocol: 05886 /06," p. 464.

Consent: "They signed a consent form, according to the Helsinki II Declaration and the Dentistry Ethical Code (CONEP/ MS, Brazil)," p. 464.

\section{Participants}

Description of ID used: "moderate mental retardation ("trainable" category, intelligence quotient 40 to 55)," p. 464. Down Syndrome.

Conversion to ICD description: mild to moderate

Age range: $7-13$ years

Sex: mixed

Comorbidity reported/details: exclusion criteria - excluded if any systemic disease

Number of participants at baseline: 40

Number of participants at final evaluation: 40

Selection of the participants for the intervention: participants were "invited to participate"

Interventions

\section{Comparison: disclosing agent versus no disclosing agent}

Carers invited for instruction on oral hygiene. Provided with a child's toothbrush, test toothpaste and detailed instructions. Instructed to brush 3 times a day. During the washout phase, carers returned to usual habits, using fluoridated toothpaste and toothbrush

Group 1: fluoridated toothpaste

Group 2: fluoridated toothpaste with plaque-disclosing agent

Group 3: fluoridated toothpaste + chlorhexidine

Group 4: fluoridated toothpaste + chlorhexidine + plaque-disclosing agent

We used only data from groups 1 and 2. Groups 3 and 4 were not eligible for inclusion in this review.

1. Plaque Index (Green \& Vermillion) (V)
2. Gingival Bleeding Index (Ainamo \& Bay) (V)
Timing of outcome assessments: baseline and day 10 for each of 4 phases

COM-B System Characteristics

Potential sources of behaviour change: capability (physical/psychological), opportunity (physical), motivation (reflective)

Potential Intervention functions: training, enablement, environmental restructuring

Stakeholder Involvement

Formal or non-formal carer: both

With or without dental professional involvement: with - dentist, dental assistant 
Teitelbaum 2009 (Continued)

Other stakeholder involvement: parents, formal carers, participants own dentist (general dental practitioner) and dental assistant

Notes
Modifications to the intervention: none reported
Adverse effects: exclusion criteria: "an allergic reaction to any tested chemical agent," - no exclusions
reported
Funding source: "This work was supported by Fundacao Araucaria de Apoio ao Desenvolvimento Cien-
tıfico e Tecnologico do Parana, Brazil," p. 467.

\section{Risk of bias}

\begin{tabular}{|c|c|c|}
\hline Bias & Authors' judgement & Support for judgement \\
\hline $\begin{array}{l}\text { Random sequence genera- } \\
\text { tion (selection bias) }\end{array}$ & Unclear risk & Comment: no details provided. \\
\hline $\begin{array}{l}\text { Allocation concealment } \\
\text { (selection bias) }\end{array}$ & Unclear risk & Comment: no details provided. \\
\hline $\begin{array}{l}\text { Blinding of participants } \\
\text { and personnel (perfor- } \\
\text { mance bias) } \\
\text { All outcomes }\end{array}$ & Low risk & $\begin{array}{l}\text { Quote: "The experimental dentifrices were packed into plain white 50-g plastic } \\
\text { tubes and coded according (to) each group." "Parents did not know which of } \\
\text { the four experimental dentifrices they were using for their children," p. } 465 . \\
\text { Comment: probably done. }\end{array}$ \\
\hline $\begin{array}{l}\text { Blinding of outcome as- } \\
\text { sessment (detection bias) } \\
\text { All outcomes }\end{array}$ & Unclear risk & $\begin{array}{l}\text { Quote: "Clinical examinations were performed by a previously trained examin- } \\
\text { er," p. } 465 . \\
\text { Comment: unclear if "blind" trial refers to the assessor or the parents, p. } 464 \text {. }\end{array}$ \\
\hline $\begin{array}{l}\text { Incomplete outcome data } \\
\text { (attrition bias) } \\
\text { All outcomes }\end{array}$ & Low risk & Comment: no missing data. \\
\hline
\end{tabular}

Selective reporting (re- Unclear risk Comment: no protocol but all listed outcomes reported. porting bias)

\section{Williams 1988}

\begin{tabular}{ll}
\hline Methods & Study design: NRCT \\
Date of study: not stated, pre-1988 \\
Study duration: 3 months \\
Location: USA \\
Setting: residential \\
Ethical approval: none reported \\
Consent: none reported
\end{tabular}


Williams 1988 (Continued)

Conversion to ICD description: profound

Age range: 4-36 years

Sex: male 9, female 15

Comorbidity reported/details: none reported

Number of participants at baseline: 24

Number of participants at final evaluation: unclear

Selection of the participants for the intervention: participants were "selected."

\section{Interventions Comparison: special manual toothbrush versus conventional manual toothbrush}

Group 1: brushed daily with Collis Curve toothbrush by student dental assistant using the Collis Curve Scrub Method

Group 2: brushed daily with conventional toothbrush by student dental assistant using the Modified Stillman Method

$\begin{array}{ll}\text { Outcomes } & \text { 1. Simplified Green \& Vermillion Oral Hygiene Index - plaque only (scale 0-3) (V) } \\ & \text { Timing of outcome assessments: baseline and } 3 \text { months }\end{array}$

COM-B System Character- Potential sources of behaviour change: opportunity (physical)
istics

Potential Intervention functions: environmental restructuring

\begin{tabular}{ll} 
Stakeholder Involvement & $\begin{array}{l}\text { Formal or non-formal carer: formal } \\
\text { With or without dental professional involvement: with - student dental assi } \\
\text { Other stakeholder involvement: none reported }\end{array}$ \\
\hline Notes & Strengths and weaknesses: brushing carried out by a dental professional \\
Modifications to the intervention: none reported \\
Adverse effects: none reported \\
Funding source: none reported
\end{tabular}

\section{Risk of bias}

\begin{tabular}{lll}
\hline Bias & Authors' judgement & Support for judgement \\
\hline $\begin{array}{l}\text { Random sequence genera- } \\
\text { tion (selection bias) }\end{array}$ & Unclear risk & $\begin{array}{l}\text { Quote: "equally divided into control and experimental groups," p. } 2 . \\
\text { Comment: no evidence of randomisation. }\end{array}$ \\
\hline $\begin{array}{l}\text { Allocation concealment } \\
\text { (selection bias) }\end{array}$ & Unclear risk & Comment: no details provided. \\
\hline $\begin{array}{l}\text { Blinding of participants } \\
\text { and personnel (perfor- } \\
\text { mance bias) } \\
\text { All outcomes }\end{array}$ & High risk & Comment: not possible to blind personnel. \\
\hline
\end{tabular}

\footnotetext{
Blinding of outcome as-

Unclear risk

Comment: data collected by the dentist in the facility; unclear if blinded, p. 2 . sessment (detection bias)
} 
Williams 1988 (Continued)

All outcomes

Incomplete outcome data Low risk Comment: assumed no missing data, p. 292.
(attrition bias)

All outcomes

Selective reporting (re- Unclear risk $\quad$ Comment: no protocol but all listed outcomes reported.
porting bias)

porting bias)

API: Approximal Plaque Index; ICD: International Statistical Classification of Diseases and Related Health Problems; ID: intellectual disability; IQ: intelligence quotient; ITS: interrupted time series; N/A: not available; NRCT: non-randomised controlled trial; OHI: oral hygiene instruction; RCT: randomised controlled trial; SD: standard deviation; V: verified; WHO: World Health Organization.

\section{Characteristics of excluded studies [ordered by study ID]}

\begin{tabular}{ll}
\hline Study & Reason for exclusion \\
\hline Andrews 1990 & $\begin{array}{l}\text { Conference abstract only. Data in a format that could not be used. Efforts to contact authors were } \\
\text { unsuccessful. }\end{array}$ \\
\hline Badra 1973 & Title only. All attempts to source full text and contact authors were unsuccessful. \\
\hline Borglin 1969 & Title only. Efforts to source full article and contact authors were unsuccessful. \\
\hline Brody 1975 & $\begin{array}{l}\text { Unclear data in the published report regarding study design. Efforts to contact authors were unsuc- } \\
\text { cessful. }\end{array}$ \\
\hline Bui 2003 & $\begin{array}{l}1 \text { subgroup possibly eligible for inclusion. Contact with 2nd author (Antonia Scott) - study did not } \\
\text { meet the required study design. }\end{array}$
\end{tabular}

Favell $1975 \quad$ Relevant data were not presented in the published report. Attempts to contact the author were un-
successful.

$\begin{array}{ll}\text { Gertenrich } 1972 & \text { Data in published report was in a format that could not be used. Contact with the author - data } \\ \text { were no longer available. }\end{array}$

\begin{tabular}{ll}
\hline Haran 2014 & Contact with author - study did not meet the required study design. \\
\hline Horner 1975 & $\begin{array}{l}\text { Interrupted time series study with unclear data; } 2 \text { participants appeared to have only } 2 \text { baseline } \\
\text { measures; postintervention time frame was different for each participant; no clear finish time } \\
\text { point. Unable to contact the author to clarify. }\end{array}$ \\
\hline ISRCTN10044161 & $\begin{array}{l}\text { Trial registration only. Thesis published by the author. Unable to contact the author or access the } \\
\text { thesis. }\end{array}$ \\
\hline Kaschke 2008 & Contact with author - study did not meet the required study design. \\
\hline Lesmana 2014 & Abstract only. Contact made with author - data no longer available. \\
\hline Lopez 1994 & Title only. Unable to contact the author or access the article. \\
\hline Meador 1979 & No data provided in the published report. Unable to contact the author. \\
\hline NCT03234231 & $\begin{array}{l}\text { Trial registration only. Contact with authors - study was not progressing due to difficulties recruit- } \\
\text { ing participants. }\end{array}$ \\
\hline
\end{tabular}




\begin{tabular}{ll}
\hline Study & Reason for exclusion \\
\hline NCT03791385 & $\begin{array}{l}\text { Authors confirmed that the study was completed, but did not include a population with intellectual } \\
\text { disabilities. }\end{array}$ \\
\hline Ojeda 2010 & $\begin{array}{l}\text { Conference abstract only. Data were in a format that could not be used. Unable to contact the au- } \\
\text { thors. }\end{array}$ \\
\hline Ribeiro 2011 & $\begin{array}{l}\text { Conference abstract. Not the mechanical control of plaque only. Subgroup may have been eligible } \\
\text { for inclusion. Unable to contact authors. }\end{array}$ \\
\hline Schmidt 1981 & $\begin{array}{l}\text { Data in the published report were in a format that could not be used. Contact made with author (JP } \\
\text { O'Donnell) - data no longer available. }\end{array}$ \\
\hline Thornton 1991 & Conference abstract. Data were in a format that could not be used. Unable to contact the author. \\
\hline Zaksek 2014 & Did not meet the required study design. \\
\hline Zhou 2019 & Before-and-after study - only 1 pre- and postintervention assessment reported - did not meet re- \\
\hline
\end{tabular}

Characteristics of studies awaiting assessment [ordered by study ID]

Akgun 2012

Methods

Study design: controlled trial; details to be confirmed

Date of study: not given, pre-2012

Study duration: 24 months

Setting: home

Ethical approval: unknown

Consent: unknown

Participants

Description of ID used: mental disability

Conversion to ICD description: unclear

Age: unknown

Sex: unknown

Comorbidity reported/details: unknown

Number of participants at baseline: 112

Number of participants at final evaluation: unknown

Selection of participants for the intervention: participants were attending 2 special schools

Country: Turkey

\section{Comparison: carer oral health training versus no oral health training}

Group 1: carer oral health training including oral hygiene instruction and dietary counselling (4 weeks)

Group 2: no carer oral health training 


\begin{tabular}{ll}
\hline Outcomes & Plaque Index \\
2. Gingival Index \\
3. DMFT \\
4. DMFS \\
Timing of outcome assessments: baseline and 24 months \\
\hline Notes & Potential sources of behaviour change: capability (physical/psychological), motivation (reflective) \\
& Potential intervention functions: education, training, enablement \\
\hline
\end{tabular}

Birani 2008

Methods

Study design: controlled trial; exact details to be confirmed

Date of study: not given, pre-2008

Study duration: 90 days

Setting: unknown

Ethical approval: unknown

Consent: unknown

Description of ID used: severe neuropsychomotor disabilities/mants
Conversion to ICD description: severe
Age: unknown
Sex: unknown
Comorbidity reported/details: unknown
Number of participants at baseline: 78
Number of participants at final evaluation: unknown
Selection of the participants for the intervention: unknown
Country: Brazil

Interventions

Comparison: oral health audiovisual material for guidance and motivation of carers versus no oral health audiovisual material

Group 1: 1 video display to the carers + oral hygiene promotion

Group 2: oral hygiene promotion only

\begin{tabular}{ll}
\hline Outcomes & 1. Visible Plaque Index \\
& 2. Gingival Bleeding Index \\
& Timing of outcome assessments: baseline, 15, 30, 60 and 90 days \\
\hline Notes & Potential sources of behaviour change: capability (physical/psychological), motivation (reflective) \\
\hline
\end{tabular}


Birani 2008 (Continued)

Potential Intervention functions: education, training, modelling, enablement, environmental restructuring

Saptiwi 2018

\begin{tabular}{ll}
\hline Methods & Non-randomised controlled study - pretest post-test design \\
\hline Participants & $\begin{array}{l}76 \text { children with special needs ("mentally disabled") at "public elementary school for exceptional } \\
\text { children" in Semarang, Indonesia }\end{array}$ \\
\hline
\end{tabular}

\begin{tabular}{ll}
\hline Interventions & Donuts Oral Health School Innovative Program - education, training, monitoring \\
\hline Outcomes & Oral hygiene index, dental and oral healthcare maintenance \\
\hline Notes & Funding: Health Polytechnic Ministry of Health, Semarang, Indonesia \\
\hline
\end{tabular}

DMFS: Decayed, Missing or Filled Surface; DMFT: Decayed, Missing or Filled Teeth; ICD: International Statistical Classification of Diseases and Related Health Problems; ID: intellectual disability.

Characteristics of ongoing studies [ordered by study ID]

NCT03522337

\begin{tabular}{ll}
\hline Trial name or title & $\begin{array}{l}\text { Effectiveness of visual pedagogy-assisted tooth-brushing training among preschoolers with special } \\
\text { needs for oral health promotion }\end{array}$ \\
\hline Methods & Double-blind RCT \\
\hline Participants & $\begin{array}{l}\text { Children aged 2-6 years attending "Special Child Care Centres" in Hong Kong } \\
\text { ity; requiring emergent dental treatment; use of antibiotic within preceding } 3 \text { months; dental pro- } \\
\text { phylaxis in preceding } 6 \text { months }\end{array}$ \\
\hline Interventions & $\begin{array}{l}\text { Social stories about toothbrushing versus conventional information leaflet } \\
\text { Outcomes }\end{array}$ \\
$\begin{array}{l}\text { Change in: carious tooth surfaces, caries prevalence, oral hygiene status, gingival status, tooth- } \\
\text { brushing performance, eating habits, dental visit experience, amount of toothpaste and tooth- } \\
\text { brush wear }\end{array}$ \\
\hline $\begin{array}{l}\text { 12 April } 2016 \\
\text { Contact information }\end{array}$ \\
$\begin{array}{l}\text { Principal investigator: Dr Gloria Hai-Ming Wong, The University of Hong Kong wonghmg@hku.hk } \\
\text { Quote: "The randomised sequence will be generated by an investigator who does not participate } \\
\text { in the outreach service. The allocation sequence will be sealed in an envelope, and opened at the } \\
\text { terials to children and their parents." }\end{array}$ \\
\hline
\end{tabular}

$\mathrm{RCT}$ : randomised controlled trial. 


\section{DATA AND ANALYSES}

Comparison 1. Special manual toothbrush (TB) versus conventional manual TB (used by carers) for people with intellectual disabilities (ID)

\begin{tabular}{|c|c|c|c|c|}
\hline Outcome or subgroup title & No. of studies & $\begin{array}{l}\text { No. of partici- } \\
\text { pants }\end{array}$ & Statistical method & Effect size \\
\hline $\begin{array}{l}1 \text { Gingival inflammation short } \\
\text { term (<6 weeks) }\end{array}$ & 1 & 25 & $\begin{array}{l}\text { Mean Difference (IV, Fixed, 95\% } \\
\mathrm{CI})\end{array}$ & $-0.10[-0.77,0.57]$ \\
\hline $\begin{array}{l}2 \text { Gingival inflammation medium } \\
\text { term ( } 6 \text { weeks to } 12 \text { months) }\end{array}$ & 1 & 18 & $\begin{array}{l}\text { Mean Difference (IV, Fixed, 95\% } \\
\mathrm{CI})\end{array}$ & $\begin{array}{l}-12.40[-24.31 \\
-0.49]\end{array}$ \\
\hline 3 Plaque short term (< 6 weeks) & 1 & 25 & $\begin{array}{l}\text { Mean Difference (IV, Fixed, 95\% } \\
\mathrm{CI})\end{array}$ & $0.20[-0.45,0.85]$ \\
\hline $\begin{array}{l}4 \text { Plaque medium term ( } 6 \text { weeks } \\
\text { to } 12 \text { months) }\end{array}$ & 1 & 18 & $\begin{array}{l}\text { Mean Difference (IV, Fixed, 95\% } \\
\mathrm{CI})\end{array}$ & $-0.44[-0.93,0.05]$ \\
\hline 5 Oral health assessments & 1 & & $\begin{array}{l}\text { Mean Difference (IV, Fixed, 95\% } \\
\mathrm{CI})\end{array}$ & Subtotals only \\
\hline 5.1 Oral assessment & 1 & 22 & $\begin{array}{l}\text { Mean Difference (IV, Fixed, 95\% } \\
\mathrm{CI})\end{array}$ & $-0.45[-1.35,0.45]$ \\
\hline 5.2 Total bacteria count & 1 & 22 & $\begin{array}{l}\text { Mean Difference (IV, Fixed, 95\% } \\
\mathrm{CI})\end{array}$ & $0.32[-0.29,0.93]$ \\
\hline $\begin{array}{l}5.3 \text { Potentially pathogenic bacte- } \\
\text { ria }\end{array}$ & 1 & 22 & $\begin{array}{l}\text { Mean Difference (IV, Fixed, 95\% } \\
\mathrm{CI} \text { ) }\end{array}$ & $0.12[-0.67,0.91]$ \\
\hline $\begin{array}{l}6 \text { Behaviour, attitude and self-ef- } \\
\text { ficacy }\end{array}$ & 1 & & $\begin{array}{l}\text { Mean Difference (IV, Fixed, 95\% } \\
\mathrm{CI} \text { ) }\end{array}$ & Subtotals only \\
\hline 6.1 Frequency of brushing & 1 & 18 & $\begin{array}{l}\text { Mean Difference (IV, Fixed, 95\% } \\
\mathrm{CI})\end{array}$ & $0.20[-2.13,2.53]$ \\
\hline $\begin{array}{l}6.2 \text { Experience of carer when } \\
\text { brushing for a person with ID }\end{array}$ & 1 & 18 & $\begin{array}{l}\text { Mean Difference (IV, Fixed, 95\% } \\
\mathrm{CI} \text { ) }\end{array}$ & $0.7[-0.15,1.55]$ \\
\hline 6.3 Handling of TB by the carer & 1 & 18 & $\begin{array}{l}\text { Mean Difference (IV, Fixed, 95\% } \\
\mathrm{CI} \text { ) }\end{array}$ & $0.50[-0.35,1.35]$ \\
\hline $\begin{array}{l}7 \text { Degree of resistance of person } \\
\text { with ID }\end{array}$ & 1 & 18 & $\begin{array}{l}\text { Mean Difference (IV, Fixed, 95\% } \\
\mathrm{Cl} \text { ) }\end{array}$ & $-1.70[-4.90,1.50]$ \\
\hline
\end{tabular}

\section{Analysis 1.1. Comparison 1 Special manual toothbrush (TB) versus conventional manual TB (used by carers) for people with intellectual disabilities (ID), Outcome 1 Gingival inflammation short term ( 6 weeks).}

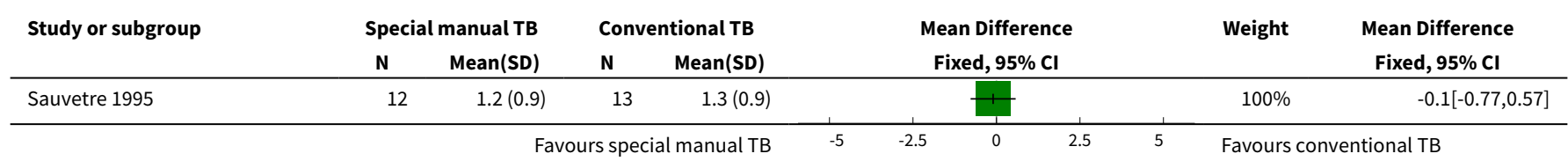




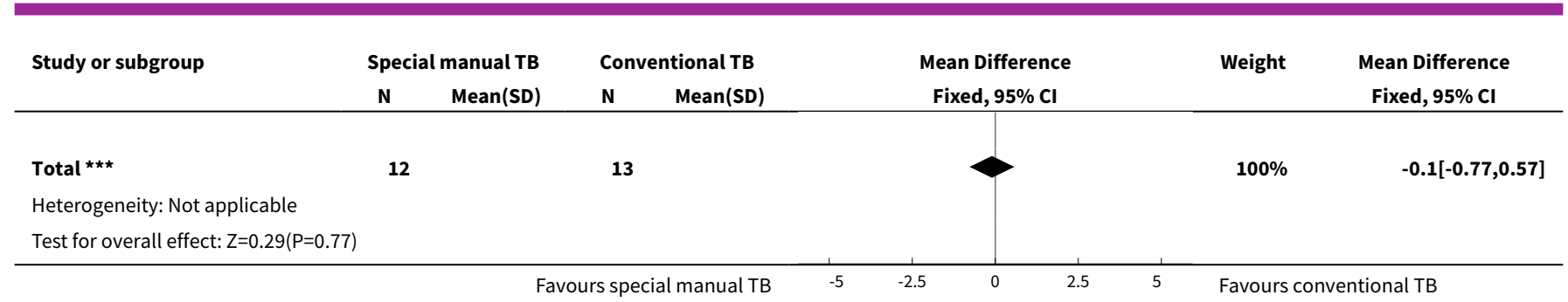

Analysis 1.2. Comparison 1 Special manual toothbrush (TB) versus conventional manual TB (used by carers) for people with intellectual disabilities (ID), Outcome 2 Gingival inflammation medium term (6 weeks to 12 months).

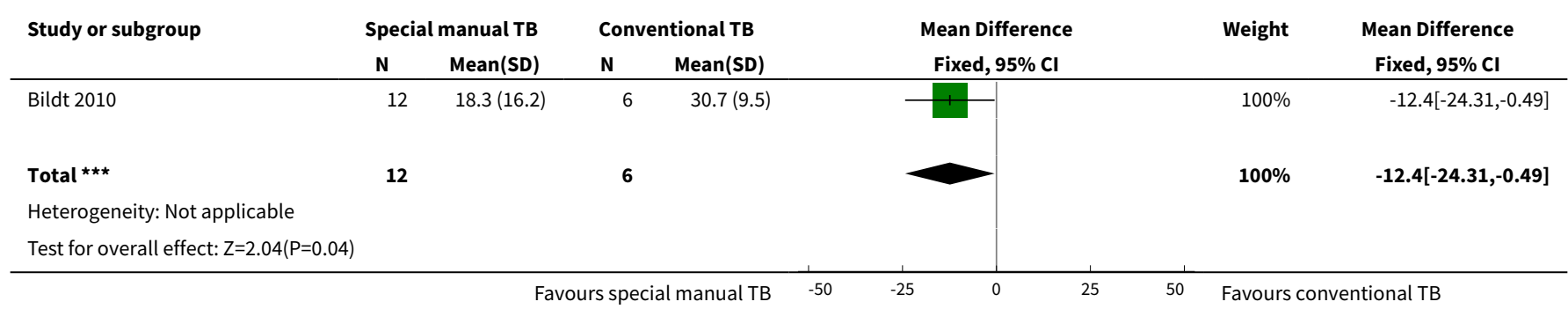

Analysis 1.3. Comparison 1 Special manual toothbrush (TB) versus conventional manual TB (used by carers) for people with intellectual disabilities (ID), Outcome 3 Plaque short term ( 6 weeks).

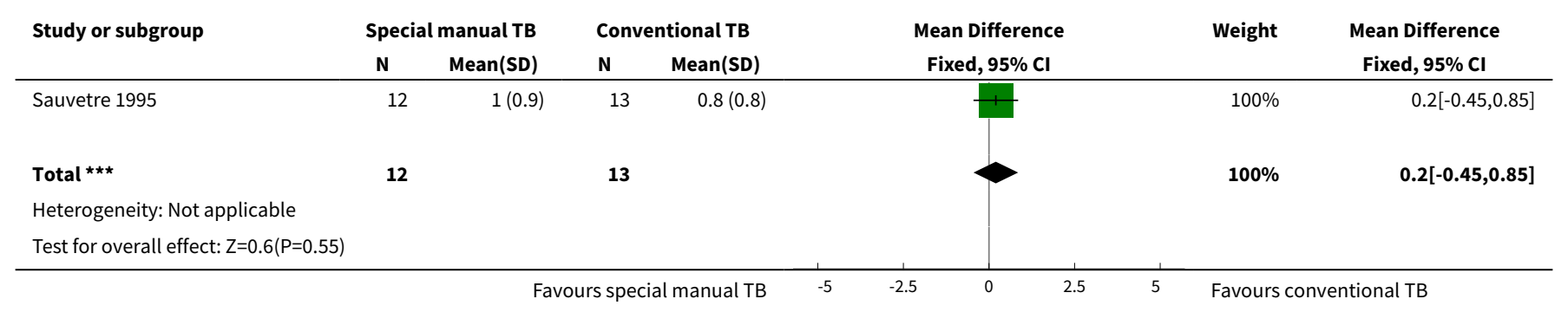

Analysis 1.4. Comparison 1 Special manual toothbrush (TB) versus conventional manual TB (used by carers) for people with intellectual disabilities (ID), Outcome 4 Plaque medium term (6 weeks to 12 months).

\begin{tabular}{|c|c|c|c|c|c|c|c|}
\hline \multirow[t]{2}{*}{ Study or subgroup } & \multicolumn{2}{|c|}{ Special manual TB } & \multicolumn{2}{|c|}{ Conventional TB } & \multirow{2}{*}{$\begin{array}{c}\text { Mean Difference } \\
\text { Fixed, } 95 \% \mathrm{Cl}\end{array}$} & \multirow[t]{2}{*}{ Weight } & \multirow{2}{*}{$\begin{array}{c}\text { Mean Difference } \\
\text { Fixed, } 95 \% \mathrm{Cl}\end{array}$} \\
\hline & $\mathbf{N}$ & $\operatorname{Mean}(\mathrm{SD})$ & $\mathbf{N}$ & Mean(SD) & & & \\
\hline Bildt 2010 & 12 & $0.8(0.7)$ & 6 & $1.2(0.4)$ & 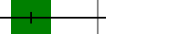 & $100 \%$ & $-0.44[-0.93,0.05]$ \\
\hline Total $\star \star \star ~$ & 12 & & 6 & & & $100 \%$ & $-0.44[-0.93,0.05]$ \\
\hline \multicolumn{8}{|c|}{ Heterogeneity: $\mathrm{Tau}^{2}=0 ; \mathrm{Chi}^{2}=0, \mathrm{df}=0(\mathrm{P}<0.0001) ; \mathrm{I}^{2}=100 \%$} \\
\hline \multicolumn{3}{|c|}{ Test for overall effect: $Z=1.74(P=0.08)$} & & & & & \\
\hline
\end{tabular}


Analysis 1.5. Comparison 1 Special manual toothbrush (TB) versus conventional manual TB (used by carers) for people with intellectual disabilities (ID), Outcome 5 Oral health assessments.

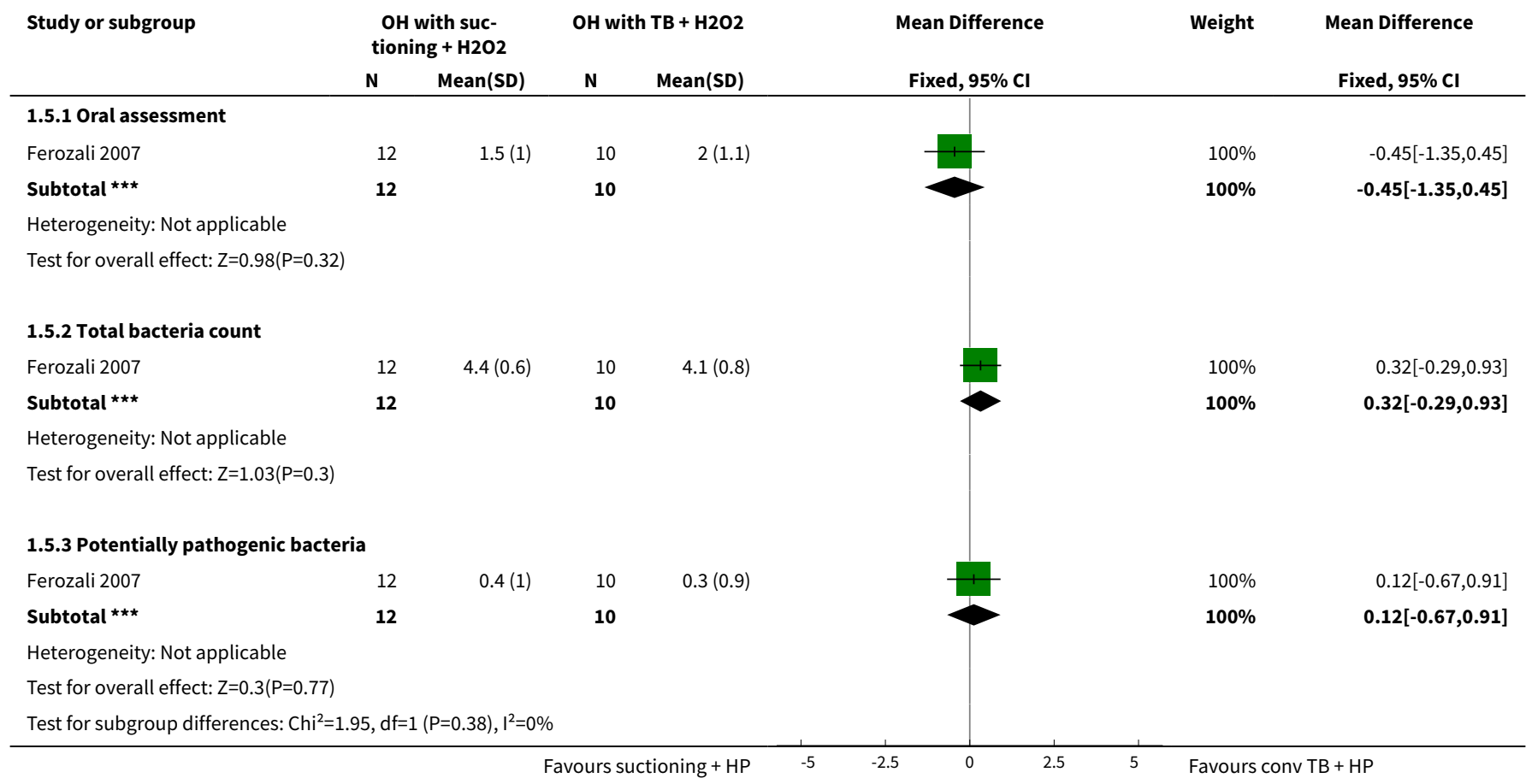

Analysis 1.6. Comparison 1 Special manual toothbrush (TB) versus conventional manual TB (used by carers) for people with intellectual disabilities (ID), Outcome 6 Behaviour, attitude and self-efficacy.

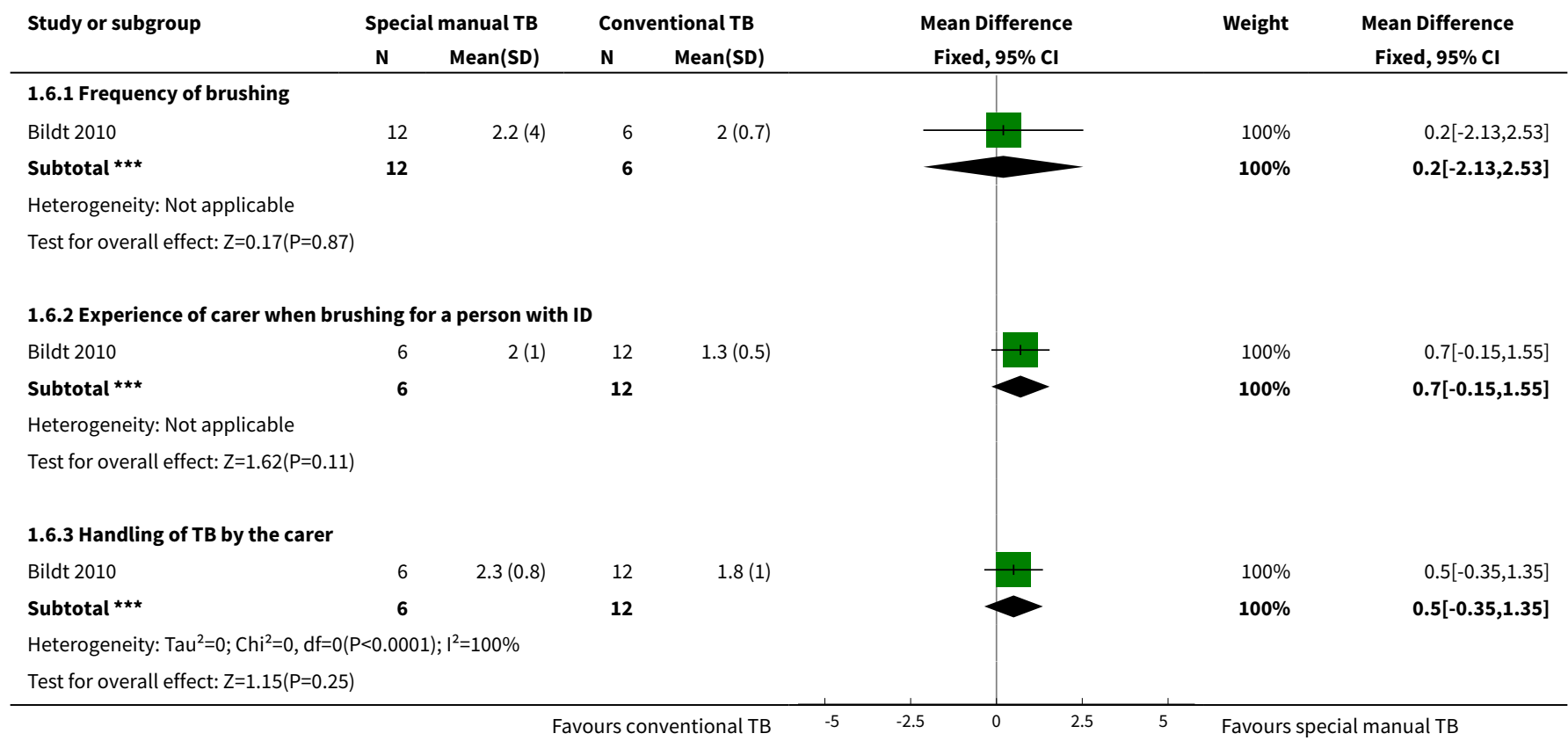


Analysis 1.7. Comparison 1 Special manual toothbrush (TB) versus conventional manual TB (used by carers) for people with intellectual disabilities (ID), Outcome 7 Degree of resistance of person with ID.

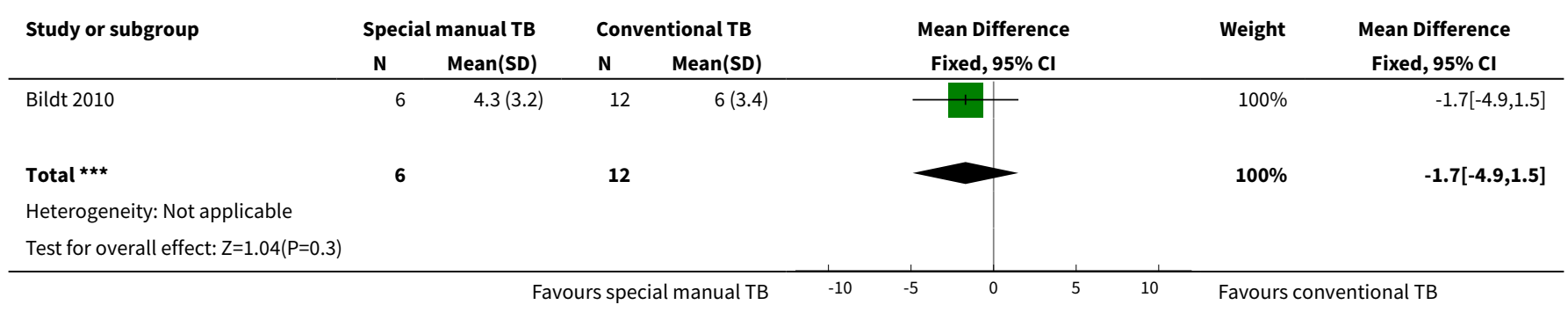

Comparison 2. Electric toothbrush (ТВ) versus manual TB for people with intellectual disabilities

\begin{tabular}{|c|c|c|c|c|}
\hline Outcome or subgroup title & No. of studies & $\begin{array}{l}\text { No. of partici- } \\
\text { pants }\end{array}$ & Statistical method & Effect size \\
\hline $\begin{array}{l}1 \text { Gingival inflammation medium term } \\
\text { ( } 6 \text { weeks to } 12 \text { months) }\end{array}$ & 2 & 120 & $\begin{array}{l}\text { Mean Difference (IV, Fixed, } \\
95 \% \mathrm{CI})\end{array}$ & $0.02[-0.06,0.09]$ \\
\hline $\begin{array}{l}2 \text { Plaque medium term ( } 6 \text { weeks to } 12 \\
\text { months) }\end{array}$ & 2 & 120 & $\begin{array}{l}\text { Std. Mean Difference (IV, } \\
\text { Fixed, } 95 \% \mathrm{CI} \text { ) }\end{array}$ & $0.29[-0.07,0.65]$ \\
\hline $\begin{array}{l}3 \text { NRS Gingival inflammation long term } \\
\text { (> } 12 \text { months) }\end{array}$ & 1 & 23 & $\begin{array}{l}\text { Mean Difference (IV, Fixed, } \\
95 \% \mathrm{Cl} \text { ) }\end{array}$ & $0.0[-0.15,0.15]$ \\
\hline $\begin{array}{l}4 \text { NRS Plaque medium term ( } 6 \text { weeks } \\
\text { to } 12 \text { months) }\end{array}$ & 5 & 167 & $\begin{array}{l}\text { Std. Mean Difference (IV, Ran- } \\
\text { dom, } 95 \% \mathrm{Cl} \text { ) }\end{array}$ & $0.04[-0.38,0.46]$ \\
\hline $\begin{array}{l}5 \text { Calculus medium term ( } 6 \text { weeks to } 12 \\
\text { months) }\end{array}$ & 2 & 120 & $\begin{array}{l}\text { Std. Mean Difference (IV, } \\
\text { Fixed, } 95 \% \mathrm{CI} \text { ) }\end{array}$ & $-0.04[-0.40,0.32]$ \\
\hline
\end{tabular}

Analysis 2.1. Comparison 2 Electric toothbrush (TB) versus manual TB for people with intellectual disabilities, Outcome 1 Gingival inflammation medium term (6 weeks to 12 months).

\begin{tabular}{|c|c|c|c|c|c|c|c|}
\hline \multirow[t]{2}{*}{ Study or subgroup } & \multicolumn{2}{|c|}{ Electric TB } & \multicolumn{2}{|c|}{ Manual TB } & \multirow{2}{*}{$\begin{array}{c}\text { Mean Difference } \\
\text { Fixed, } 95 \% \mathrm{Cl}\end{array}$} & \multirow[t]{2}{*}{ Weight } & \multirow{2}{*}{$\begin{array}{c}\text { Mean Difference } \\
\text { Fixed, } 95 \% \mathrm{Cl}\end{array}$} \\
\hline & $\mathbf{N}$ & Mean(SD) & $\mathbf{N}$ & Mean(SD) & & & \\
\hline Carr 1997 & 31 & $1.9(0.2)$ & 25 & $1.9(0.2)$ & & $67.92 \%$ & $0[-0.09,0.09]$ \\
\hline Garcia-Carrillo 2016 & 32 & $1.2(0.3)$ & 32 & $1.2(0.3)$ & - & $32.08 \%$ & $0.05[-0.09,0.19]$ \\
\hline Total $\star \star \star ~$ & 63 & & 57 & & & $100 \%$ & $0.02[-0.06,0.09]$ \\
\hline \multicolumn{8}{|c|}{ Heterogeneity: Tau $^{2}=0 ; \mathrm{Chi}^{2}=0.35, \mathrm{df}=1(\mathrm{P}=0.56) ; \mathrm{I}^{2}=0 \%$} \\
\hline \multicolumn{8}{|c|}{ Test for overall effect: $Z=0.4(P=0.69)$} \\
\hline
\end{tabular}


Analysis 2.2. Comparison 2 Electric toothbrush (TB) versus manual TB for people with intellectual disabilities, Outcome 2 Plaque medium term (6 weeks to 12 months).

\begin{tabular}{|c|c|c|c|c|c|c|c|}
\hline \multirow[t]{2}{*}{ Study or subgroup } & \multicolumn{2}{|c|}{ Electric TB } & \multicolumn{2}{|c|}{ Manual TB } & \multirow{2}{*}{$\begin{array}{c}\text { Std. Mean Difference } \\
\text { Fixed, } 95 \% \mathrm{Cl}\end{array}$} & \multirow[t]{2}{*}{ Weight } & \multirow{2}{*}{$\begin{array}{l}\text { Std. Mean Difference } \\
\text { Fixed, } 95 \% \mathrm{Cl}\end{array}$} \\
\hline & $\mathbf{N}$ & Mean(SD) & $\mathbf{N}$ & Mean(SD) & & & \\
\hline Garcia-Carrillo 2016 & 32 & $1.1(0.4)$ & 32 & $1(0.4)$ & 5 & $53.89 \%$ & $0.23[-0.27,0.72]$ \\
\hline Carr 1997 & 31 & $1.6(0.7)$ & 25 & $1.3(0.4)$ & + & $46.11 \%$ & $0.36[-0.17,0.9]$ \\
\hline Total $\star \star \star ~$ & 63 & & 57 & & $\nabla$ & $100 \%$ & $0.29[-0.07,0.65]$ \\
\hline \multicolumn{8}{|c|}{ Heterogeneity: $\mathrm{Tau}^{2}=0 ; \mathrm{Chi}^{2}=0.14, \mathrm{df}=1(\mathrm{P}=0.71) ; \mathrm{I}^{2}=0 \%$} \\
\hline
\end{tabular}

Analysis 2.3. Comparison 2 Electric toothbrush (TB) versus manual TB for people with intellectual disabilities, Outcome 3 NRS Gingival inflammation long term (>12 months).

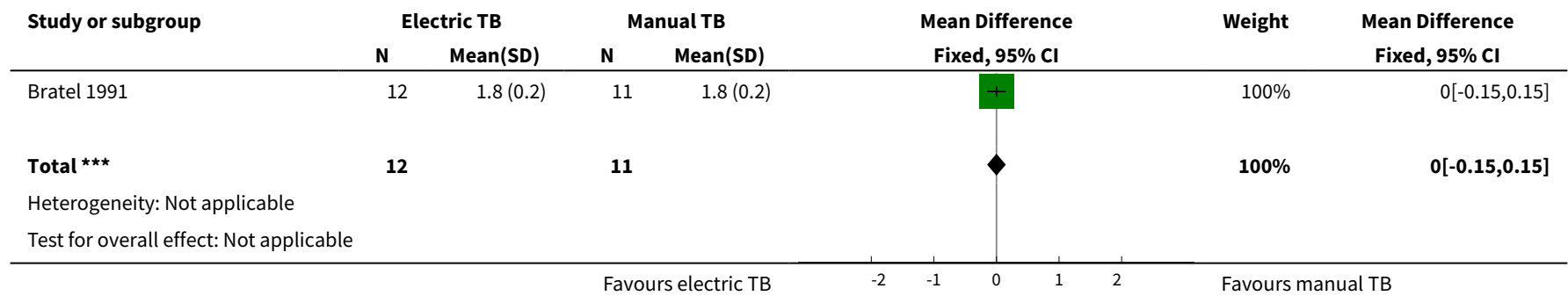

Analysis 2.4. Comparison 2 Electric toothbrush (TB) versus manual TB for people with intellectual disabilities, Outcome 4 NRS Plaque medium term ( 6 weeks to 12 months).

\begin{tabular}{|c|c|c|c|c|c|c|c|}
\hline \multirow{3}{*}{$\begin{array}{l}\text { Study or subgroup } \\
\text { Bratel } 1991\end{array}$} & \multicolumn{2}{|c|}{ Electric TB } & \multicolumn{2}{|c|}{ Manual TB } & \multirow{2}{*}{$\begin{array}{c}\text { Std. Mean Difference } \\
\text { Random, } 95 \% \mathrm{Cl}\end{array}$} & \multirow[t]{2}{*}{ Weight } & \multirow{2}{*}{$\begin{array}{c}\text { Std. Mean Difference } \\
\text { Random, } 95 \% \mathrm{Cl}\end{array}$} \\
\hline & $\mathbf{N}$ & $\operatorname{Mean}(S D)$ & $\mathbf{N}$ & Mean(SD) & & & \\
\hline & 12 & $1.1(0.1)$ & 11 & $1.1(0.3)$ & & $17.13 \%$ & $-0.04[-0.86,0.78]$ \\
\hline Gertenrich 1967a & 7 & $-0.7(0.6)$ & 7 & $-0.8(0.7)$ & & $12.12 \%$ & $0.12[-0.93,1.17]$ \\
\hline Gertenrich 1967b & 34 & $0.2(0.6)$ & 18 & $0(1.4)$ & + & $25.44 \%$ & $0.23[-0.35,0.8]$ \\
\hline Gertenrich 1967c & 18 & $0.4(0.5)$ & 18 & $0.7(0.5)$ & & $21.58 \%$ & $-0.67[-1.35,0]$ \\
\hline Gertenrich 1967d & 21 & $-0.6(0.5)$ & 21 & $-0.9(0.7)$ & 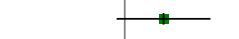 & $23.73 \%$ & $0.51[-0.11,1.12]$ \\
\hline 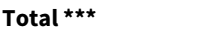 & 92 & & 75 & & & $100 \%$ & $0.04[-0.38,0.46]$ \\
\hline \multicolumn{8}{|c|}{ Heterogeneity: $\mathrm{Tau}^{2}=0.1 ; \mathrm{Chi}^{2}=6.97, \mathrm{df}=4(\mathrm{P}=0.14) ; \mathrm{I}^{2}=42.64 \%$} \\
\hline \multicolumn{8}{|c|}{ Test for overall effect: $Z=0.19(P=0.85)$} \\
\hline
\end{tabular}

Analysis 2.5. Comparison 2 Electric toothbrush (TB) versus manual TB for people with intellectual disabilities, Outcome 5 Calculus medium term (6 weeks to 12 months).

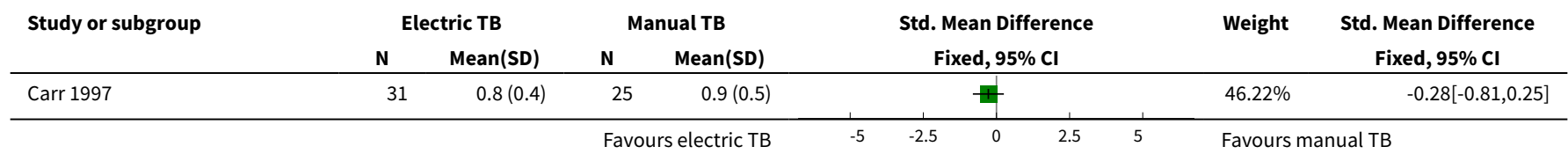




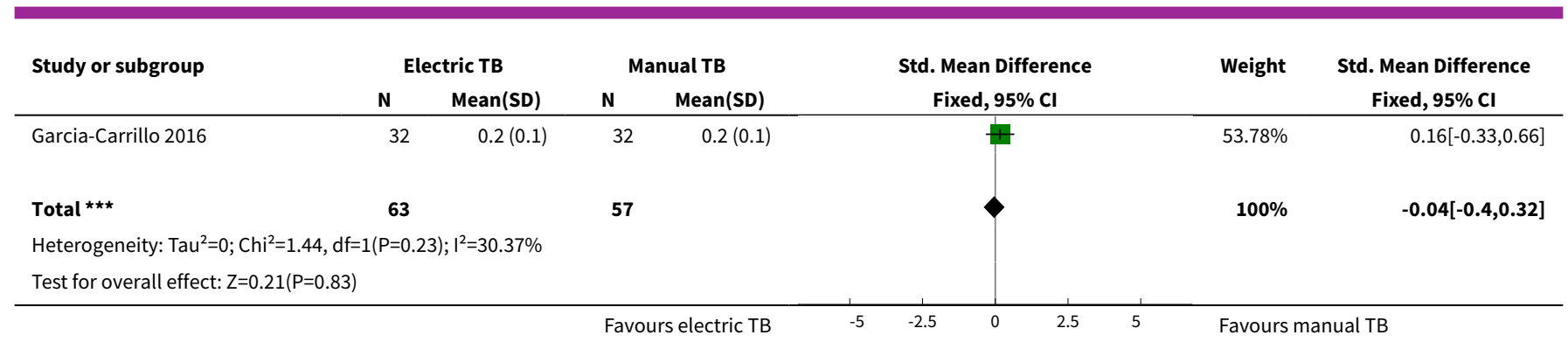

Comparison 3. Training of carers of people with intellectual disabilities versus no training of carers

\begin{tabular}{|c|c|c|c|c|}
\hline Outcome or subgroup title & No. of studies & $\begin{array}{l}\text { No. of partici- } \\
\text { pants }\end{array}$ & Statistical method & Effect size \\
\hline $\begin{array}{l}1 \text { Gingival inflammation medium } \\
\text { term ( } 6 \text { weeks to } 12 \text { months) }\end{array}$ & 2 & 99 & $\begin{array}{l}\text { Mean Difference (IV, Random, } \\
95 \% \mathrm{CI} \text { ) }\end{array}$ & $-0.09[-0.63,0.45]$ \\
\hline $\begin{array}{l}2 \text { Plaque medium term ( } 6 \text { weeks to } 12 \\
\text { months) }\end{array}$ & 2 & 99 & $\begin{array}{l}\text { Mean Difference (IV, Fixed, } \\
95 \% \mathrm{CI})\end{array}$ & $-0.07[-0.26,0.13]$ \\
\hline $\begin{array}{l}3 \text { Non-randomised study plaque } \\
\text { short term ( }<6 \text { weeks) }\end{array}$ & 1 & & $\begin{array}{l}\text { Mean Difference (IV, Fixed, } \\
95 \% \mathrm{CI})\end{array}$ & Subtotals only \\
\hline 3.1 Training of carers & 1 & 34 & $\begin{array}{l}\text { Mean Difference (IV, Fixed, } \\
95 \% \mathrm{CI})\end{array}$ & $-0.69[-0.97,-0.41]$ \\
\hline 4 Knowledge short term (< 6 weeks) & 1 & & $\begin{array}{l}\text { Mean Difference (IV, Fixed, } \\
95 \% \mathrm{Cl})\end{array}$ & Subtotals only \\
\hline $\begin{array}{l}5 \text { Knowledge medium term ( } 6 \text { weeks } \\
\text { to } 12 \text { months) }\end{array}$ & 2 & 189 & $\begin{array}{l}\text { Mean Difference (IV, Fixed, } \\
95 \% \mathrm{Cl} \text { ) }\end{array}$ & $0.69[0.31,1.06]$ \\
\hline $\begin{array}{l}6 \text { Behaviour, attitude and self-effica- } \\
\text { cy medium term }\end{array}$ & 2 & 189 & $\begin{array}{l}\text { Mean Difference (IV, Random, } \\
95 \% \mathrm{CI} \text { ) }\end{array}$ & $0.15[-0.80,1.10]$ \\
\hline
\end{tabular}

\section{Analysis 3.1. Comparison 3 Training of carers of people with intellectual disabilities versus no training of carers, Outcome 1 Gingival inflammation medium term (6 weeks to 12 months).}

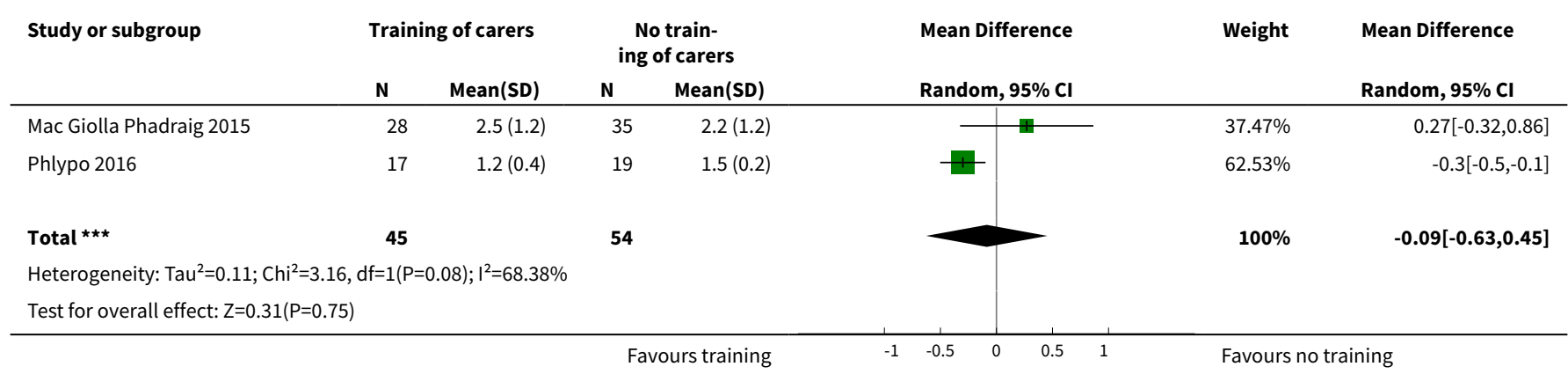


Analysis 3.2. Comparison 3 Training of carers of people with intellectual disabilities versus no training of carers, Outcome 2 Plaque medium term (6 weeks to 12 months).

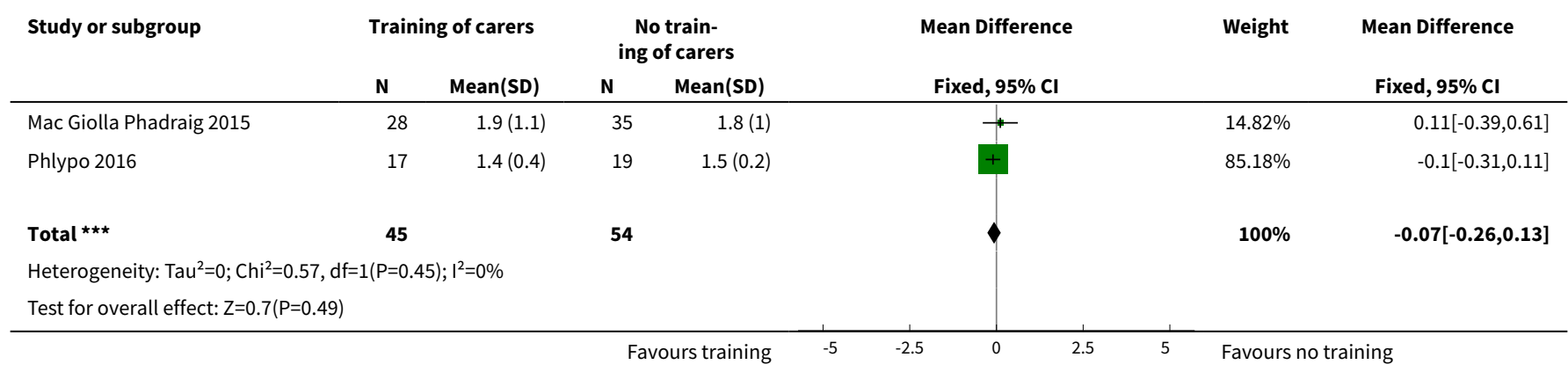

Analysis 3.3. Comparison 3 Training of carers of people with intellectual disabilities versus no training of carers, Outcome 3 Non-randomised study plaque short term ( $<6$ weeks).

\begin{tabular}{|c|c|c|c|c|c|c|c|}
\hline \multirow[t]{2}{*}{ Study or subgroup } & \multicolumn{2}{|c|}{ Training of carers } & \multicolumn{2}{|c|}{$\begin{array}{l}\text { No train- } \\
\text { ing of carers }\end{array}$} & \multirow{2}{*}{$\begin{array}{c}\text { Mean Difference } \\
\text { Fixed, } 95 \% \mathrm{Cl} \\
\end{array}$} & \multirow[t]{2}{*}{ Weight } & \multirow{2}{*}{$\begin{array}{l}\text { Mean Difference } \\
\text { Fixed, } 95 \% \mathrm{Cl}\end{array}$} \\
\hline & $\mathbf{N}$ & $\operatorname{Mean}(\mathrm{SD})$ & $\mathbf{N}$ & Mean(SD) & & & \\
\hline \multicolumn{8}{|c|}{ 3.3.1 Training of carers } \\
\hline Lange 2000 & 22 & $1.1(0.3)$ & 12 & $1.8(0.5)$ & & $100 \%$ & $-0.69[-0.97,-0.41]$ \\
\hline 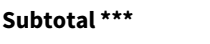 & 22 & & 12 & & & $100 \%$ & $-0.69[-0.97,-0.41]$ \\
\hline \multicolumn{8}{|c|}{ Heterogeneity: Not applicable } \\
\hline
\end{tabular}

Analysis 3.4. Comparison 3 Training of carers of people with intellectual disabilities versus no training of carers, Outcome 4 Knowledge short term ( $<6$ weeks).

\begin{tabular}{|c|c|c|c|c|c|c|c|c|c|}
\hline \multirow[t]{2}{*}{ Study or subgroup } & \multicolumn{2}{|c|}{ Training of carers } & \multicolumn{2}{|c|}{$\begin{array}{c}\text { No train- } \\
\text { ing of carers }\end{array}$} & \multicolumn{3}{|c|}{ Mean Difference } & \multirow[t]{2}{*}{ Weight } & \multirow{2}{*}{$\begin{array}{l}\text { Mean Difference } \\
\text { Fixed, } 95 \% \mathrm{Cl}\end{array}$} \\
\hline & $\mathbf{N}$ & $\operatorname{Mean}(S D)$ & $\mathbf{N}$ & Mean(SD) & & Fixed, $95 \% \mathrm{Cl}$ & & & \\
\hline Gonzalez 2013 & 14 & $18.3(2)$ & 10 & $17.7(1.5)$ & & + & & $0 \%$ & $0.59[-0.8,1.98]$ \\
\hline
\end{tabular}

Analysis 3.5. Comparison 3 Training of carers of people with intellectual disabilities versus no training of carers, Outcome 5 Knowledge medium term (6 weeks to 12 months).

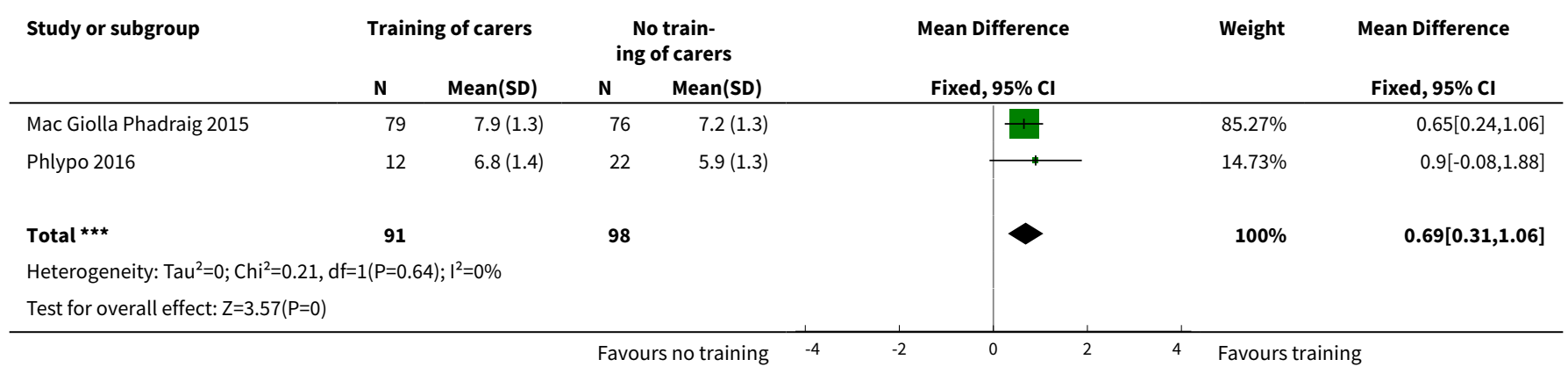


Analysis 3.6. Comparison 3 Training of carers of people with intellectual disabilities versus no training of carers, Outcome 6 Behaviour, attitude and self-efficacy medium term.

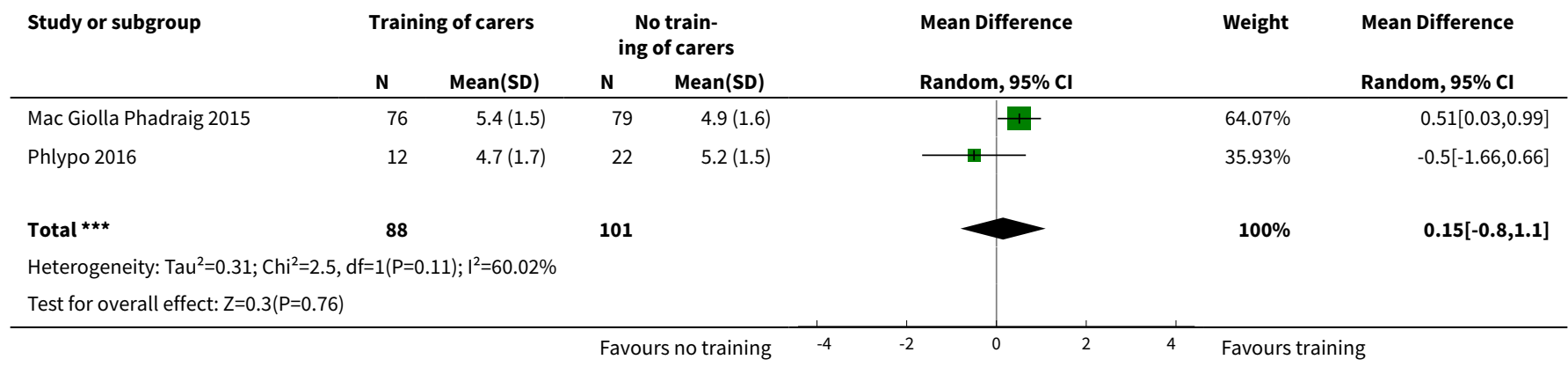

Comparison 4. Oral health $(\mathrm{OH})$ training of people with intellectual disabilities (ID) versus no training

\begin{tabular}{lllll}
\hline Outcome or subgroup title & No. of studies & $\begin{array}{l}\text { No. of partici- } \\
\text { pants }\end{array}$ & Statistical method & Effect size \\
\hline $\begin{array}{l}1 \text { Gingival inflammation short term } \\
(<6 \text { weeks) }\end{array}$ & 1 & 10 & $\begin{array}{l}\text { Mean Difference (IV, Fixed, 95\% } \\
\text { Cl) }\end{array}$ & $-0.28[-0.90,0.34]$ \\
\hline 2 Plaque short term $(<6$ weeks) & 1 & 10 & $\begin{array}{l}\text { Mean Difference (IV, Fixed, 95\% } \\
\text { Cl) }\end{array}$ & $-0.47[-0.92,-0.02]$
\end{tabular}

Analysis 4.1. Comparison 4 Oral health $(\mathrm{OH})$ training of people with intellectual disabilities (ID) versus no training, Outcome 1 Gingival inflammation short term (< 6 weeks).

\begin{tabular}{|c|c|c|c|c|c|c|c|}
\hline \multirow[t]{2}{*}{ Study or subgroup } & \multicolumn{2}{|c|}{$\begin{array}{l}\text { OH training of } \\
\text { people with ID }\end{array}$} & \multicolumn{2}{|c|}{$\begin{array}{l}\text { No OH training } \\
\text { of people with ID }\end{array}$} & \multirow{2}{*}{$\begin{array}{c}\text { Mean Difference } \\
\text { Fixed, } 95 \% \mathrm{Cl} \\
\end{array}$} & \multirow[t]{2}{*}{ Weight } & \multirow{2}{*}{$\begin{array}{l}\text { Mean Difference } \\
\text { Fixed, } 95 \% \mathrm{Cl}\end{array}$} \\
\hline & $\mathbf{N}$ & Mean(SD) & $\mathbf{N}$ & Mean(SD) & & & \\
\hline Lange 1985 & 5 & $1(0.5)$ & 5 & $1.3(0.5)$ & - & $100 \%$ & $-0.28[-0.9,0.34]$ \\
\hline Total $\star \star \star$ & 5 & & 5 & & & $100 \%$ & $-0.28[-0.9,0.34]$ \\
\hline \multicolumn{8}{|c|}{ Heterogeneity: Not applicable } \\
\hline
\end{tabular}

Analysis 4.2. Comparison 4 Oral health $(\mathrm{OH})$ training of people with intellectual disabilities (ID) versus no training, Outcome 2 Plaque short term (< 6 weeks).

\begin{tabular}{|c|c|c|c|c|c|c|c|}
\hline \multirow[t]{2}{*}{ Study or subgroup } & \multicolumn{2}{|c|}{$\begin{array}{l}\text { OH training of } \\
\text { people with ID }\end{array}$} & \multicolumn{2}{|c|}{$\begin{array}{l}\text { No OH training } \\
\text { of people with ID }\end{array}$} & \multirow{2}{*}{$\begin{array}{c}\text { Mean Difference } \\
\text { Fixed, } 95 \% \mathrm{CI}\end{array}$} & \multirow[t]{2}{*}{ Weight } & \multirow{2}{*}{$\begin{array}{c}\text { Mean Difference } \\
\text { Fixed, } 95 \% \mathrm{Cl}\end{array}$} \\
\hline & $\mathbf{N}$ & $\operatorname{Mean}(S D)$ & $\mathbf{N}$ & Mean(SD) & & & \\
\hline Lange 1985 & 5 & $0.4(0.5)$ & 5 & $0.8(0.2)$ & 1 & $100 \%$ & $-0.47[-0.92,-0.02]$ \\
\hline
\end{tabular}




\begin{tabular}{|c|c|c|c|c|c|}
\hline \multirow[t]{2}{*}{ Study or subgroup } & $\begin{array}{l}\text { OH training of } \\
\text { people with ID }\end{array}$ & $\begin{array}{l}\text { No OH training } \\
\text { of people with ID }\end{array}$ & \multirow{2}{*}{$\begin{array}{c}\text { Mean Difference } \\
\text { Fixed, } 95 \% \mathrm{Cl}\end{array}$} & \multirow[t]{2}{*}{ Weight } & \multirow{2}{*}{$\begin{array}{c}\text { Mean Difference } \\
\text { Fixed, } 95 \% \mathrm{Cl}\end{array}$} \\
\hline & Mean(SD) & $\operatorname{Mean}(S D)$ & & & \\
\hline 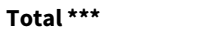 & 5 & 5 & & $100 \%$ & $-0.47[-0.92,-0.02]$ \\
\hline \multicolumn{6}{|c|}{ Heterogeneity: Tau $^{2}=0 ; \mathrm{Chi}^{2}=0, \mathrm{df}=0(\mathrm{P}<0.0001) ; \mathrm{I}^{2}=100 \%$} \\
\hline \multicolumn{6}{|c|}{ Test for overall effect: $Z=2.03(P=0.04)$} \\
\hline
\end{tabular}

\section{Comparison 5. Dental recall intervals for people with intellectual disabilities}

\begin{tabular}{|c|c|c|c|c|}
\hline Outcome or subgroup title & No. of studies & $\begin{array}{l}\text { No. of partici- } \\
\text { pants }\end{array}$ & Statistical method & Effect size \\
\hline $\begin{array}{l}1 \text { Gingival pocketing long term } \\
\text { (> } 12 \text { months) }\end{array}$ & 1 & & Mean Difference (IV, Fixed, 95\% CI) & Subtotals only \\
\hline 1.1 One-monthly dental recalls & 1 & 137 & Mean Difference (IV, Fixed, 95\% CI) & $-0.6[-0.97,-0.23]$ \\
\hline $\begin{array}{l}1.2 \text { Three-monthly dental re- } \\
\text { calls }\end{array}$ & 1 & 148 & Mean Difference (IV, Fixed, 95\% CI) & $-0.5[-0.85,-0.15]$ \\
\hline 1.3 Six-monthly dental recalls & 1 & 171 & Mean Difference (IV, Fixed, 95\% CI) & $-0.4[-0.76,-0.04]$ \\
\hline $\begin{array}{l}2 \text { Gingival bleeding long term } \\
\text { (> } 12 \text { months) }\end{array}$ & 1 & & Mean Difference (IV, Fixed, 95\% CI) & Subtotals only \\
\hline 2.1 One-monthly dental recall & 1 & 137 & Mean Difference (IV, Fixed, 95\% CI) & $-0.20[-0.86,0.46]$ \\
\hline $\begin{array}{l}2.2 \text { Three-monthly dental re- } \\
\text { calls }\end{array}$ & 1 & 148 & Mean Difference (IV, Fixed, 95\% CI) & $-0.10[-0.73,0.53]$ \\
\hline 2.3 Six-monthly dental recalls & 1 & 171 & Mean Difference (IV, Fixed, 95\% CI) & $0.20[-0.38,0.78]$ \\
\hline $\begin{array}{l}3 \text { Plaque long term (> } 12 \\
\text { months) }\end{array}$ & 1 & & Mean Difference (IV, Fixed, 95\% CI) & Subtotals only \\
\hline 3.1 One-monthly dental recall & 1 & 137 & Mean Difference (IV, Fixed, 95\% CI) & $-0.7[-1.30,-0.10]$ \\
\hline $\begin{array}{l}\text { 3.2 Three-monthly dental re- } \\
\text { call }\end{array}$ & 1 & 148 & Mean Difference (IV, Fixed, 95\% CI) & $-0.8[-1.38,-0.22]$ \\
\hline 3.3 Six-monthly dental recall & 1 & 171 & Mean Difference (IV, Fixed, 95\% CI) & $-0.7[-1.22,-0.18]$ \\
\hline $\begin{array}{l}4 \text { Calculus long term (> } 12 \\
\text { months) }\end{array}$ & 1 & & Mean Difference (IV, Fixed, 95\% CI) & Subtotals only \\
\hline 4.1 One-monthly dental recalls & 1 & 137 & Mean Difference (IV, Fixed, 95\% CI) & $-2.0[-2.64,-1.36]$ \\
\hline $\begin{array}{l}4.2 \text { Three-monthly dental re- } \\
\text { calls }\end{array}$ & 1 & 148 & Mean Difference (IV, Fixed, 95\% CI) & $-1.4[-2.04,-0.76]$ \\
\hline 4.3 Six-monthly dental recalls & 1 & 171 & Mean Difference (IV, Fixed, 95\% CI) & $-0.10[-0.72,0.52]$ \\
\hline
\end{tabular}


Analysis 5.1. Comparison 5 Dental recall intervals for people with intellectual disabilities, Outcome 1 Gingival pocketing long term (> 12 months).

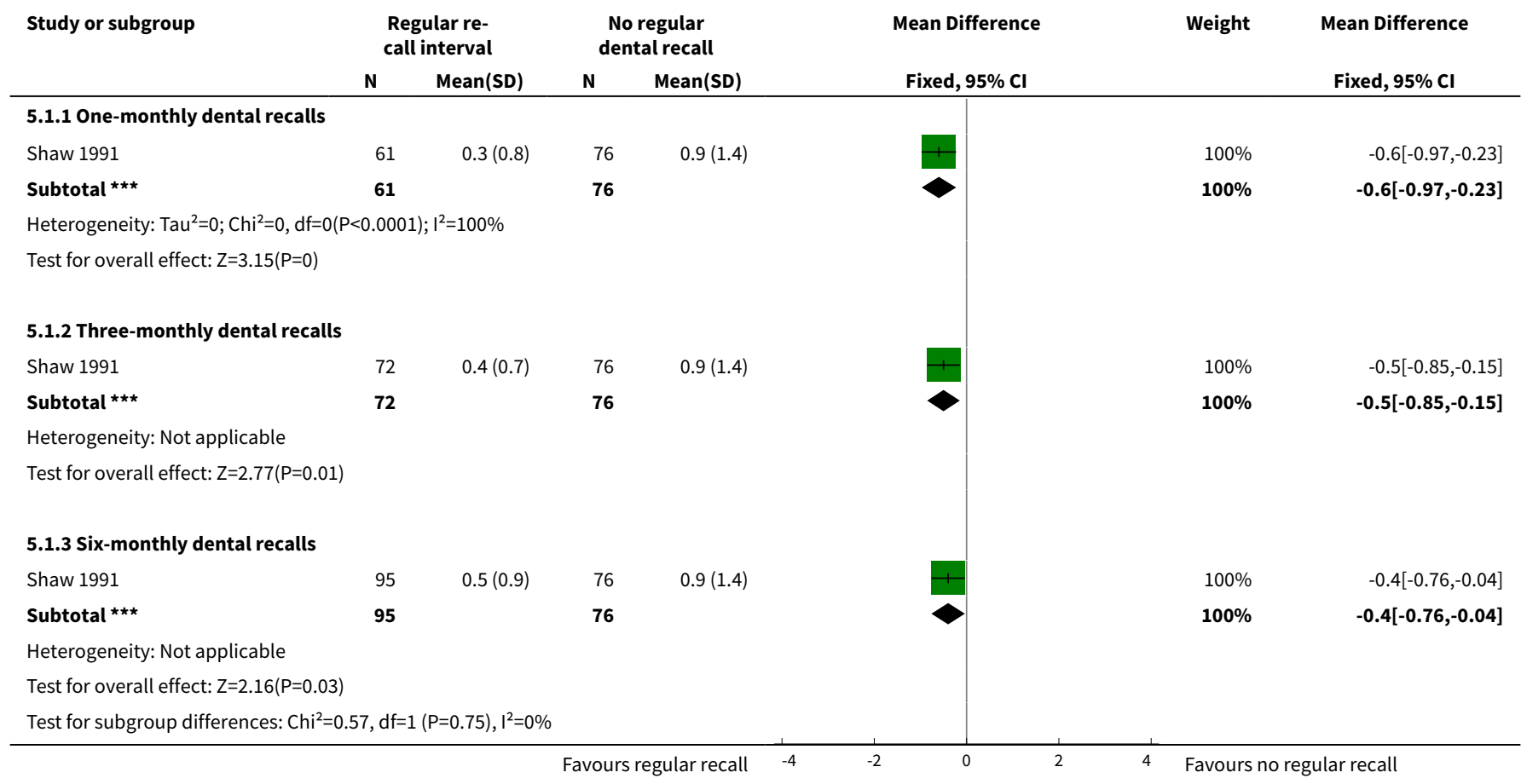

Analysis 5.2. Comparison 5 Dental recall intervals for people with intellectual disabilities, Outcome 2 Gingival bleeding long term (> 12 months).

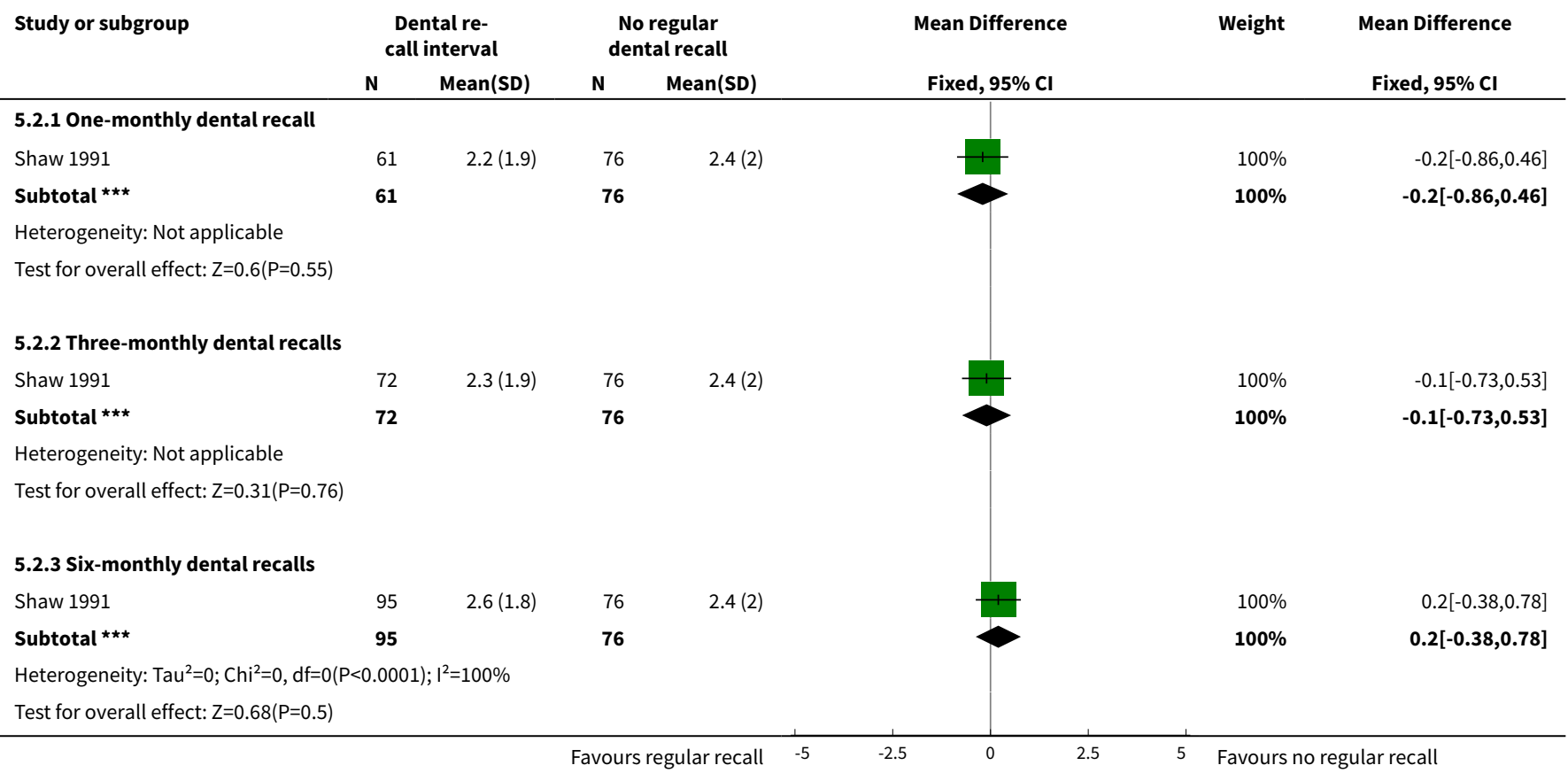


Analysis 5.3. Comparison 5 Dental recall intervals for people with intellectual disabilities, Outcome 3 Plaque long term (> 12 months).

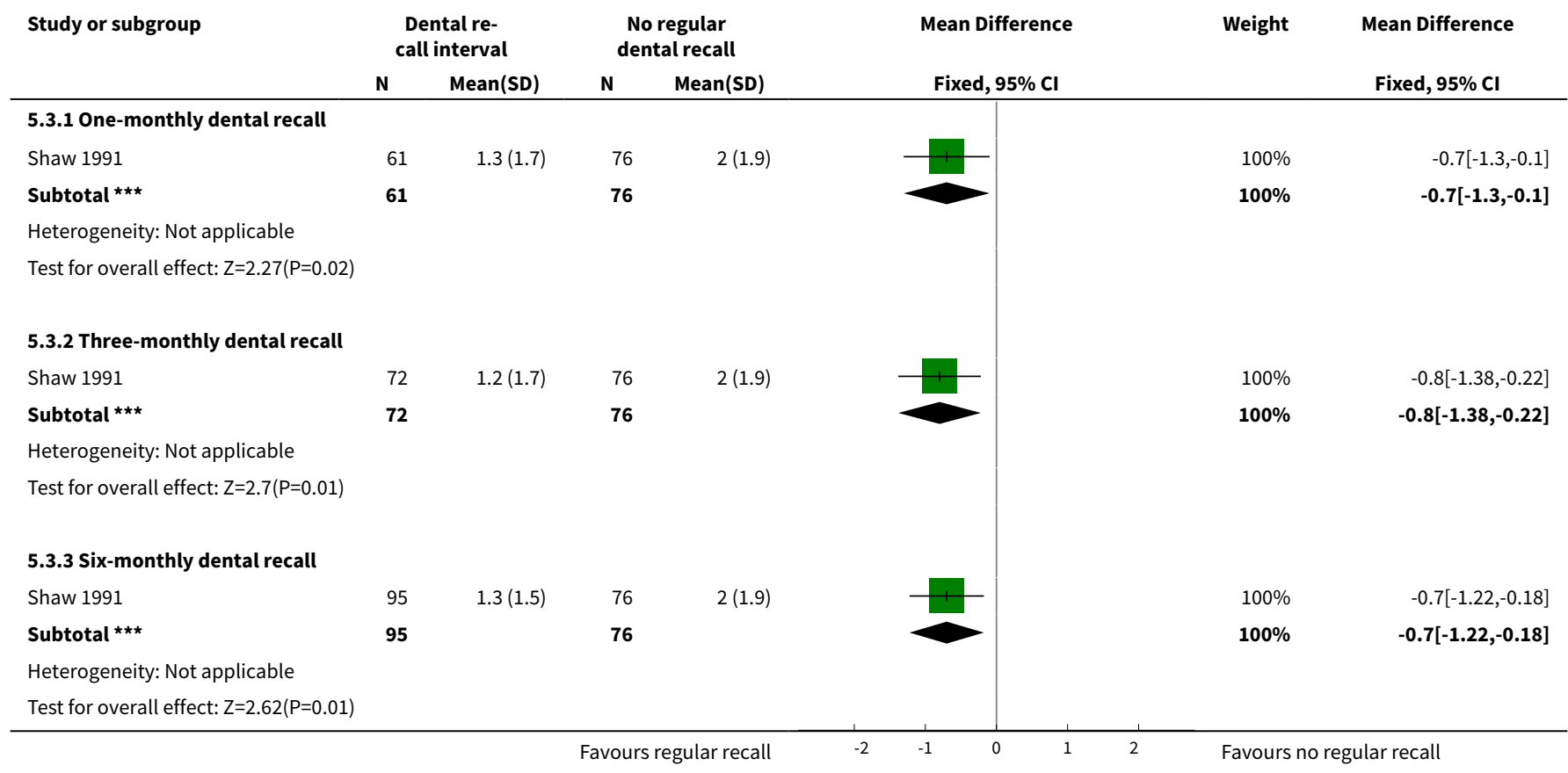

Analysis 5.4. Comparison 5 Dental recall intervals for people with intellectual disabilities, Outcome 4 Calculus long term (>12 months).

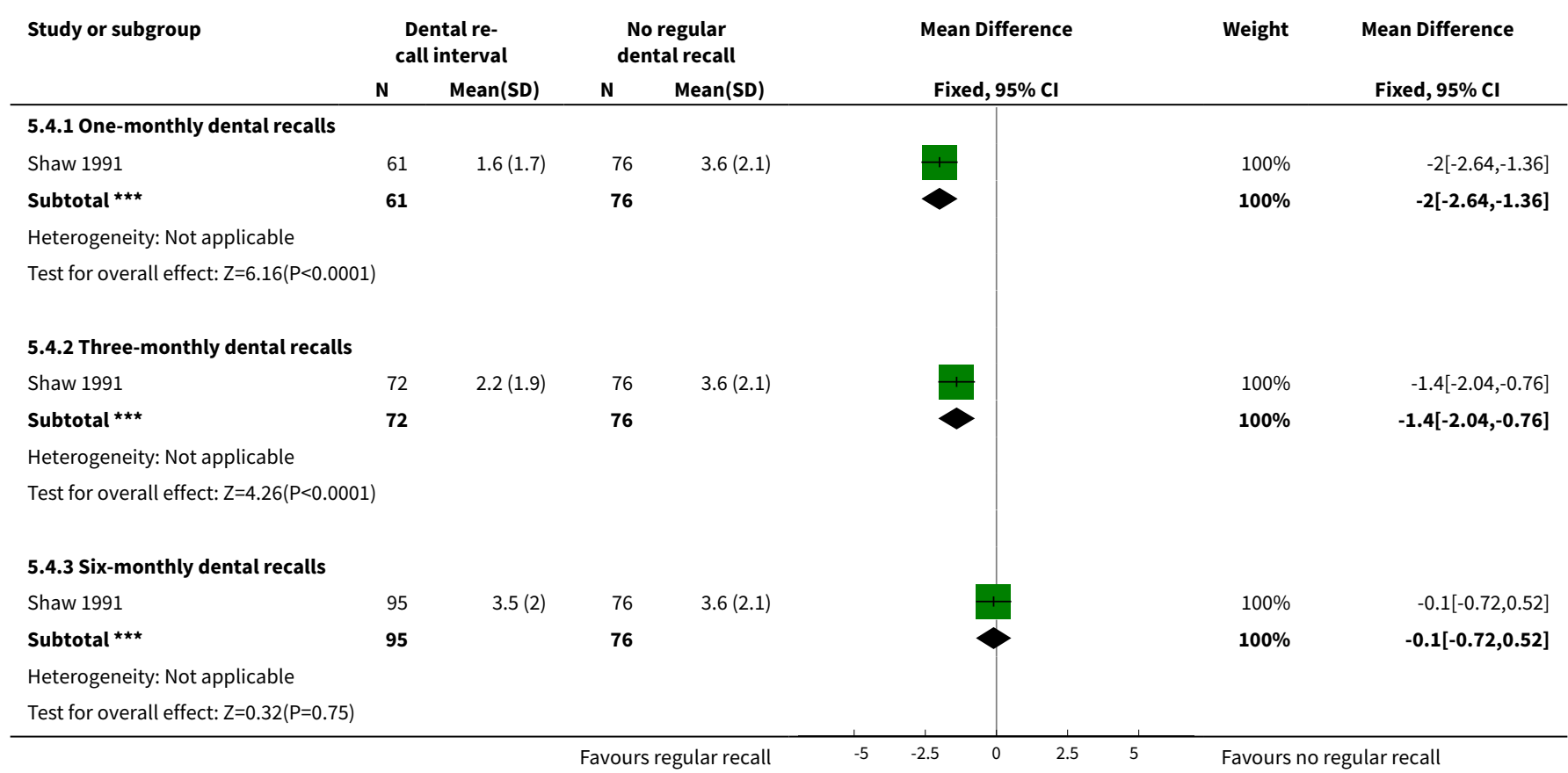


Comparison 6. Clinical photographs as motivators for oral hygiene in people with intellectual disabilities

\begin{tabular}{lllll}
\hline Outcome or subgroup title & No. of studies & $\begin{array}{l}\text { No. of partici- } \\
\text { pants }\end{array}$ & Statistical method & Effect size \\
\hline $\begin{array}{l}1 \text { Plaque medium term (6 } \\
\text { weeks to } 12 \text { months) }\end{array}$ & 1 & & Mean Difference (IV, Fixed, 95\% Cl) & Subtotals only \\
\hline 1.1 Plaque & 1 & 29 & Mean Difference (IV, Fixed, 95\% Cl) & $-0.10[-1.85,1.65]$ \\
\hline 1.2 Extrinsic stain & 1 & 29 & Mean Difference (IV, Fixed, 95\% Cl) & $-2.60[-19.45,14.25]$ \\
\hline
\end{tabular}

Analysis 6.1. Comparison 6 Clinical photographs as motivators for oral hygiene in people with intellectual disabilities, Outcome 1 Plaque medium term (6 weeks to 12 months).

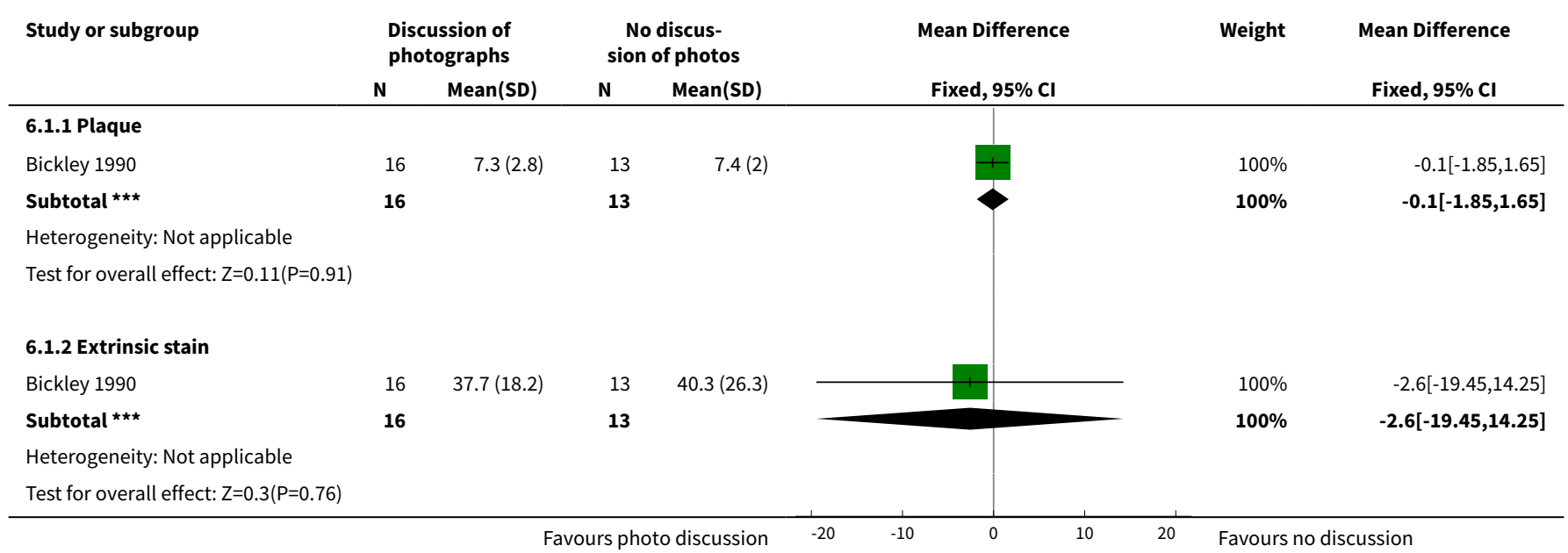


A D DITIONAL TABLES

Table 1. Special manual toothbrush versus conventional manual toothbrush (RCTs): gingival inflammation short term

\begin{tabular}{|c|c|c|c|c|c|c|c|c|c|}
\hline Duration & Study & $\begin{array}{l}\text { Compari- } \\
\text { son }\end{array}$ & Index & $\begin{array}{l}\text { Interven- } \\
\text { tion mean } \\
\text { (SD) }\end{array}$ & $\begin{array}{l}\text { Control } \\
\text { mean (SD) }\end{array}$ & Total & Country & Comments & Summary \\
\hline \multirow[t]{2}{*}{2 weeks } & $\begin{array}{l}\text { Kaschke } \\
2005\end{array}$ & $\begin{array}{l}\text { Super- } \\
\text { brush vs } \\
\text { Oral B } \\
\text { manual TB }\end{array}$ & $\begin{array}{l}\text { PBI (scale } \\
0-4)\end{array}$ & $\begin{array}{l}\text { Mouth } \\
\text { score } 3.55 \\
(0.40) \text { Sur- } \\
\text { face score } \\
7.1(0.8)\end{array}$ & $\begin{array}{l}\text { Mouth } \\
\text { score } 4.13 \\
(\mathbf{0 . 3 5 )} \text { Sur- } \\
\text { face score } \\
8.25(0.7)\end{array}$ & 54 & Germany & $\begin{array}{l}\text { Cross-over trial. Da- } \\
\text { ta presented for the } 2 \\
\text { arms combined. Sur- } \\
\text { face score mean and } \\
\text { SD obtained from au- } \\
\text { thor. Mouth score cal- } \\
\text { culated from this data. } \\
\text { Median presented in } \\
\text { published article. }\end{array}$ & $\begin{array}{l}\text { Mean for the PBI for the Super- } \\
\text { brush was below that of man- } \\
\text { ual TB. However, no strong ev- } \\
\text { idence of difference between } \\
\text { the } 2 \text { TB. }\end{array}$ \\
\hline & $\begin{array}{l}\text { Christen } \\
2007\end{array}$ & $\begin{array}{l}\text { Super- } \\
\text { brush vs } \\
\text { Oral B } \\
\text { manual TB }\end{array}$ & $\begin{array}{l}\text { PBI (scale } \\
0-4)\end{array}$ & $\begin{array}{l}\text { Pooled me- } \\
\text { dian } \mathbf{1 2 . 2} \\
\text { Self } \mathrm{Br} 10.5 \\
\text { Assist } \mathrm{Br} \\
11.5 \text { Other } \\
\mathrm{Br} 14.5\end{array}$ & $\begin{array}{l}\text { Pooled } \\
\text { median } \\
\mathbf{1 2 . 6} \text { Self } \\
\mathrm{Br} 10.0 \mathrm{As}- \\
\text { sist } \mathrm{Br} 12.5 \\
\text { Other } \mathrm{Br} \\
15.5\end{array}$ & 72 & Germany & $\begin{array}{l}\text { Cross-over trial. Da- } \\
\text { ta presented for the } \\
2 \text { arms combined. } \\
\text { Pooled median data } \\
\text { calculated from re- } \\
\text { ported data. }\end{array}$ & $\begin{array}{l}\text { "Patients who brushed their } \\
\text { teeth did not achieve signifi- } \\
\text { cant changes in PBI levels with } \\
\text { any of the three toothbrushes } \\
\text { (Wilcoxon test: } p>0.05 \text { )," p. } 63 \text {. }\end{array}$ \\
\hline 21 days & $\begin{array}{l}\text { Sauvetre } \\
1995\end{array}$ & $\begin{array}{l}\text { Super- } \\
\text { brush vs } \\
\text { standard } \\
\text { manu- } \\
\text { al brush } \\
\text { (Oral B) }\end{array}$ & $\begin{array}{l}\text { S\&MG } \\
\text { (scale 0-3) }\end{array}$ & $1.21(0.85)$ & $1.31(0.87)$ & 25 & Belgium & $\begin{array}{l}\text { Participant numbers } \\
\text { not clearly reported, } \\
\text { assumed to be } 25 \text {. }\end{array}$ & $\begin{array}{l}\text { "No significant difference was } \\
\text { found when the results of } \\
\text { bleeding indices were com- } \\
\text { pared between the two tooth- } \\
\text { brushes," p. } 117 \text {. }\end{array}$ \\
\hline
\end{tabular}

Assist Br: assisted brusher; Other Br: other brusher; PBI: Papillary Bleeding Index; RCT: randomised controlled trial; SD: standard deviation; Self Br: self-brusher; TB: toothbrush Kaschke 2005: three-armed cross-over trial; also compared the Teledyne Waterpik Sonic Speed electric TB, reported separately. Data for buccal and lingual surfaces, maxilla and mandible and Self Br, Assist Br and Other Br also reported. Christen 2007: three-armed cross-over trial; also compared the Dentacare-Sonodont electric TB, reported separately. Median and percentile data only reported. Data presented for Self Br, Assist Br and Other Br; pooled data calculated from these data. Data for buccal and lingual surfaces, maxilla and mandible also reported.

Table 2. Special manual toothbrush versus conventional manual toothbrush: gingival inflammation medium term

\begin{tabular}{lllllll}
\hline Duration Study & $\begin{array}{l}\text { Compari- Index } \\
\text { son }\end{array}$ & $\begin{array}{l}\text { Interven- } \\
\text { tion mean }\end{array}$ & $\begin{array}{l}\text { Control } \\
\text { mean (SD) }\end{array}$ & Total Country Comments & Summary
\end{tabular}

(SD) 


\begin{tabular}{|c|c|c|c|c|c|c|c|c|c|c|}
\hline 4 months & Bildt 2010 & $\begin{array}{l}\text { Super- } \\
\text { brush vs } \\
\text { Oral B } \\
\text { manual TB }\end{array}$ & $\begin{array}{l}\text { GBI (scale } \\
0-1 \text { ) }\end{array}$ & $18.3(16.2)$ & $30.7(9.5)$ & 18 & Germany & \multicolumn{2}{|c|}{$\begin{array}{l}\text { No calibration of assessors, par- } \\
\text { ticipant's own dentist assessed } \\
\text { clinical outcomes. The baseline } \\
\text { scores were considerably lower } \\
\text { for the intervention group ( } 24.7 \\
\text { vs } 32.7 \text { ). }\end{array}$} & $\begin{array}{l}\text { Author reported no } \\
\text { strong evidence of a } \\
\text { difference between the } \\
2 \text { toothbrushes for gin- } \\
\text { gival inflammation. }\end{array}$ \\
\hline \multicolumn{11}{|c|}{ GBI: Gingival Bleeding Index Ainamo \& Bay (0-1), 6 standard teeth, \% of positive sites examined; SD: standard deviation. } \\
\hline Duration & Study & $\begin{array}{l}\text { Compari- } \\
\text { son }\end{array}$ & Index & $\begin{array}{l}\text { Interven- } \\
\text { tion mean } \\
\text { (SD) }\end{array}$ & $\begin{array}{l}\text { Control } \\
\text { mean (SD) }\end{array}$ & Total & Country & Comments & \multicolumn{2}{|c|}{ Summary } \\
\hline 1 week & $\begin{array}{l}\text { Dôgan } \\
2004\end{array}$ & $\begin{array}{l}\text { Super- } \\
\text { brush vs } \\
\text { Oral B } \\
\text { Cross Ac- } \\
\text { tion } 35 / 40 \\
\text { manual TB }\end{array}$ & $\begin{array}{l}\text { MQHPI } \\
\text { (scale 0-5) }\end{array}$ & $1.77(0.62)$ & $2.15(0.58)$ & 15 & Turkey & $\begin{array}{l}\text { Cross-over trial. Data pre- } \\
\text { sented for the final time } \\
\text { point only. }\end{array}$ & \multicolumn{2}{|c|}{$\begin{array}{l}\text { Strong evidence of a differ- } \\
\text { ence in } \mathrm{PI} \text { between the Super- } \\
\text { brush and Oral } B \text { Cross Action } \\
\text { brush }(P<0.01) \text { favouring the } \\
\text { Superbrush. }\end{array}$} \\
\hline \multirow[t]{2}{*}{2 weeks } & $\begin{array}{l}\text { Christen } \\
2007\end{array}$ & $\begin{array}{l}\text { Super- } \\
\text { brush vs } \\
\text { Oral B } \\
\text { manual TB }\end{array}$ & $\begin{array}{l}\text { MQHPI } \\
\text { (scale 0-5) }\end{array}$ & $\begin{array}{l}\text { Pooled } \\
\text { median } \\
\mathbf{2 7 . 7} \\
\text { Self Br } \\
26.5 \mathrm{As}- \\
\text { sist } \mathrm{Br} 26.5 \\
\text { Other } \mathrm{Br} \\
30.0\end{array}$ & $\begin{array}{l}\text { Pooled } \\
\text { median } \\
\mathbf{2 8 . 3} \\
\text { Self Br } \\
26.0 \text { As- } \\
\text { sist } \mathrm{Br} 28.0 \\
\text { Other Br } \\
31.0\end{array}$ & 72 & Germany & $\begin{array}{l}\text { Cross-over trial. Data pre- } \\
\text { sented for the } 2 \text { arms } \\
\text { combined. Median and } \\
\text { percentile only reported. } \\
\text { Pooled median data cal- } \\
\text { culated from reported da- } \\
\text { ta. }\end{array}$ & \multicolumn{2}{|c|}{$\begin{array}{l}\text { No strong evidence of a dif- } \\
\text { ference in } \mathrm{QH} \text { median plaque } \\
\text { score across all participants } \\
\text { between the } 3 \text { different tooth- } \\
\text { brushes in the final examina- } \\
\text { tion (Friedman test: } P>0.05 \text { ), } \\
\text { p. } 55 \text {. }\end{array}$} \\
\hline & $\begin{array}{l}\text { Kaschke } \\
2005\end{array}$ & $\begin{array}{l}\text { Super- } \\
\text { brush vs } \\
\text { Oral B } \\
\text { manual TB }\end{array}$ & $\begin{array}{l}\text { MQHPI } \\
\text { (scale 0-5) }\end{array}$ & $\begin{array}{l}\text { Mouth } \\
\text { score } 2.98 \\
(\mathbf{0 . 0 8 )} \text { Sur- } \\
\text { face score } \\
35.8(0.92)\end{array}$ & $\begin{array}{l}\text { Mouth } \\
\text { score } 3.24 \\
(0.10) \text { Sur- } \\
\text { face score } \\
38.9(1.2)\end{array}$ & 54 & Germany & $\begin{array}{l}\text { Cross-over trial. Data pre- } \\
\text { sented for the } 2 \text { arms } \\
\text { combined. Surface score } \\
\text { mean and SD obtained } \\
\text { from author. Mouth score } \\
\text { calculated from these da- } \\
\text { ta. Median presented in } \\
\text { published article. Data for } \\
\text { subgroups also presented. }\end{array}$ & \multicolumn{2}{|c|}{$\begin{array}{l}\text { Plaque score for the Super- } \\
\text { brush lay below that of the } \\
\text { conventional manual TB. The } \\
\text { strength of the evidence was } \\
\text { high. }\end{array}$} \\
\hline
\end{tabular}


Table 3. Special manual toothbrush versus conventional manual toothbrush (RCTs): plaque short term (Continued)

Sauvetre
1995

Super-

brush (3-

headed)

S\&LP

vs stan-

dard man-

ual brush

(Oral B)
Participant numbers not

clearly reported: assumed

to be 25
"No significant difference was found between the two toothbrushes in the means of day 0,7 or $21, "$ p. 117

Assist Br: assisted brusher; MQHPI: Modified Quigley-Hein Plaque Index, scale of 0-5; Other Br: other brusher; PI: Plaque Index; QH: Quigley-Hein; RCT: randomised controlled trial; S\&LPI: Silness \& Löe Plaque Index, scale 0-3; SD: standard deviation; Self Br: self-brusher; TB: toothbrush.

Dôgan 2004: three-armed cross-over trial; also compared the Braun Plaque Control 3D electric TB, reported separately. Data for age groups, surfaces and approximal plaque also reported. Christen 2007: three-armed cross-over trial; also compared the Dentacare-Sonodont electric TB, reported separately. Median and percentile data only reported. Data presented for Self $\mathrm{Br}$, Assist $\mathrm{Br}$ and Other Br, pooled data were calculated from these data. Data for buccal and lingual surfaces, maxilla and mandible also reported. Kaschke 2005: three-armed cross-over trial; also compared the Teledyne Waterpik Sonic Speed electric TB, reported separately. Data for buccal and lingual surfaces, maxilla and mandible and

Self $\mathrm{Br}$, Assist $\mathrm{Br}$ and Other $\mathrm{Br}$ also reported. Some strong evidence of differences were reported in favour of the Superbrush in some of these subgroups.

Table 4. Special manual toothbrush versus conventional toothbrush (RCTs): plaque medium term

\begin{tabular}{|c|c|c|c|c|c|c|c|c|c|}
\hline Duration & Study & $\begin{array}{l}\text { Compari- } \\
\text { son }\end{array}$ & Index & $\begin{array}{l}\text { Interven- } \\
\text { tion mean } \\
\text { (SD) }\end{array}$ & $\begin{array}{l}\text { Control } \\
\text { mean (SD) }\end{array}$ & Total & Country & Comments & Summary \\
\hline 4 months & Bildt 2010 & $\begin{array}{l}\text { Super- } \\
\text { brush vs } \\
\text { Oral B } \\
\text { manual TB }\end{array}$ & $\begin{array}{l}\text { SG\&VD } \\
\text { (scale 0-3) }\end{array}$ & $\begin{array}{l}\text { Mouth } \\
\text { score } \mathbf{0 . 7 8} \\
\mathbf{( 0 . 7 )} \\
\\
\text { Tooth score } \\
4.7(4.2)\end{array}$ & $\begin{array}{l}\text { Mouth } \\
\text { score } \mathbf{1 . 2 2} \\
\mathbf{( 0 . 3 6 )} \\
\text { Tooth score } \\
7.3(2.2)\end{array}$ & 18 & Germany & $\begin{array}{l}\text { Whole mouth score calculated } \\
\text { from tooth scores. No calibra- } \\
\text { tion of assessors, participant's } \\
\text { own dentist assessed clinical } \\
\text { outcomes. The baseline score } \\
\text { was lower for the intervention } \\
\text { group ( } 8.3 \text { vs } 9.2 \text { ) }\end{array}$ & $\begin{array}{l}\text { Author reported no } \\
\text { strong evidence of a } \\
\text { difference between } \\
\text { the } 2 \text { toothbrushes } \\
\text { for plaque }(\mathrm{F}(2,15)= \\
1.20), \mathrm{P}=0.33) . \text { The- } \\
\text { sis p. } 5 \text {. }\end{array}$ \\
\hline
\end{tabular}

RCT: randomised controlled trial; Indices: SG\&VDI: Simplified Green \& Vermillion Debris Index, scale 0-18, total of 0-3 for 6 standard teeth

Table 5. Special manual toothbrush versus conventional manual toothbrush (NRS): plaque medium term

\begin{tabular}{|c|c|c|c|c|c|c|c|c|c|}
\hline Duration & Study & Comparison & Index & $\begin{array}{l}\text { Interven- } \\
\text { tion mean }\end{array}$ & Control & Total & Country & Comments & Summary \\
\hline 3 months & $\begin{array}{l}\text { Williams } \\
1988\end{array}$ & $\begin{array}{l}\text { Curved-bristle } \\
\text { manual toothbrush } \\
\text { (Collis Curve) vs } \\
\text { conventional man- } \\
\text { ual toothbrush }\end{array}$ & $\begin{array}{l}\text { SG\&VOHI } \\
\text { (scale 0-3) }\end{array}$ & 1.25 & 1.03 & 24 & USA & $\begin{array}{l}\text { SD not reported. Con- } \\
\text { tact with author - da- } \\
\text { ta no longer available. } \\
\text { Brushing carried out }\end{array}$ & $\begin{array}{l}\text { No strong evidence of a } \\
\text { difference between the } \\
2 \text { toothbrushes for the } \\
\text { oral hygiene index ( } t=- \\
0.31, P<0.76 \text { ). }\end{array}$ \\
\hline
\end{tabular}




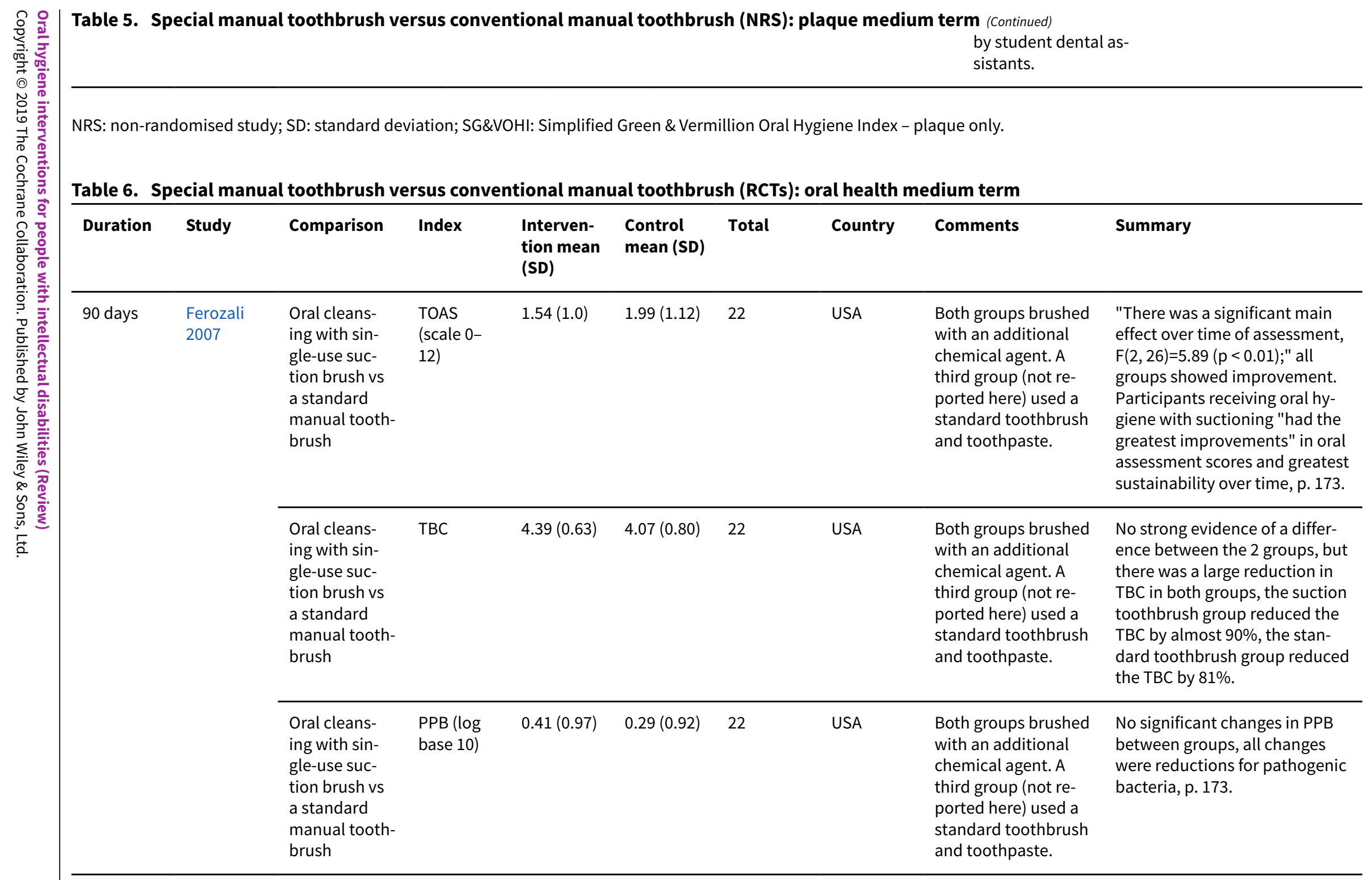

PPB: potentially pathogenic bacteria; RCT: randomised controlled trial; SD: standard deviation; TBC: Total Bacteria Count; TOAS: Total Oral Assessment Score. 
Table 7. Special manual toothbrush versus conventional manual toothbrush (RCT): behaviour, attitude and self-efficacy medium term

\begin{tabular}{|c|c|c|c|c|c|c|c|c|c|}
\hline Duration & Study & $\begin{array}{l}\text { Compari- } \\
\text { son }\end{array}$ & Index & $\begin{array}{l}\text { Interven- } \\
\text { tion mean } \\
\text { (SD) }\end{array}$ & $\begin{array}{l}\text { Control } \\
\text { mean (SD) }\end{array}$ & Total & Country & Comments & Summary \\
\hline \multirow[t]{4}{*}{4 months } & \multirow[t]{4}{*}{ Bildt 2010} & \multirow{4}{*}{$\begin{array}{l}\text { Super- } \\
\text { brush vs } \\
\text { Oral B } \\
\text { manual TB }\end{array}$} & $\begin{array}{l}\text { Frequency of } \\
\text { brushing by car- } \\
\text { er }\end{array}$ & $2.0(0.7)$ & $2.2(0.4)$ & 18 & Germany & $\begin{array}{l}\text { Questionnaire } \\
\text { not validated. }\end{array}$ & $\begin{array}{l}\text { No strong evidence of a difference } \\
\text { between TBs. }\end{array}$ \\
\hline & & & $\begin{array}{l}\text { Level of resis- } \\
\text { tance by people } \\
\text { with ID }\end{array}$ & $4.3(3.2)$ & $6.0(3.4)$ & 18 & Germany & $\begin{array}{l}\text { Questionnaire } \\
\text { not validated. }\end{array}$ & $\begin{array}{l}\text { No strong evidence of a difference } \\
\text { between TBs. }\end{array}$ \\
\hline & & & $\begin{array}{l}\text { Experience of } \\
\text { carer when } \\
\text { brushing }\end{array}$ & $2.0(1.0)$ & $1.3(0.5)$ & 18 & Germany & $\begin{array}{l}\text { Questionnaire } \\
\text { not validated. }\end{array}$ & $\begin{array}{l}\text { No strong evidence of a difference } \\
\text { between toothbrushes "although the } \\
\text { experiences appeared to be more } \\
\text { positive" for the carers using the spe- } \\
\text { cial manual TB, p. } 16 \text {. }\end{array}$ \\
\hline & & & $\begin{array}{l}\text { Handling of TB } \\
\text { by carer }\end{array}$ & $2.3(0.8)$ & $1.8(1.0)$ & 18 & Germany & $\begin{array}{l}\text { Questionnaire } \\
\text { not validated. }\end{array}$ & $\begin{array}{l}\text { No strong evidence of a difference } \\
\text { between TB. }\end{array}$ \\
\hline
\end{tabular}

ID: intellectual disability; RCT: randomised controlled trial; SD: standard deviation; TB: toothbrush.

Table 8. Electric versus manual toothbrushes (RCTs): gingival inflammation short term

\begin{tabular}{|c|c|c|c|c|c|c|c|c|c|}
\hline Duration & Study & $\begin{array}{l}\text { Compari- } \\
\text { son }\end{array}$ & Index & $\begin{array}{l}\text { Interven- } \\
\text { tion mean } \\
\text { (SD) }\end{array}$ & $\begin{array}{l}\text { Control } \\
\text { mean (SD) }\end{array}$ & Total & & Comments & Summary \\
\hline \multirow[t]{2}{*}{2 weeks } & $\begin{array}{l}\text { Kaschke } \\
2005\end{array}$ & $\begin{array}{l}\text { Teledyne } \\
\text { Waterpik } \\
\text { Sonic Speed } \\
\text { electric TB } \\
\text { vs Oral B } \\
\text { manual TB }\end{array}$ & $\begin{array}{l}\mathrm{PBI} \text { (scale } \\
0-4)\end{array}$ & $\begin{array}{l}\text { Whole } \\
\text { mouth } 3.9 \\
(0.35) \text { Sur- } \\
\text { face score } \\
7.8(0.7)\end{array}$ & $\begin{array}{l}\text { Whole } \\
\text { mouth } 4.3 \\
(0.35) \text { Sur- } \\
\text { face score } \\
8.25(0.7)\end{array}$ & 54 & Germany & $\begin{array}{l}\text { Cross-over trial. Data } \\
\text { presented for the } 2 \\
\text { arms combined. Sur- } \\
\text { face score Mean and } \\
\text { SD obtained from au- } \\
\text { thor. Data calculated } \\
\text { from obtained data. } \\
\text { Median data present- } \\
\text { ed in published arti- } \\
\text { cle. }\end{array}$ & $\begin{array}{l}\text { Although there was a great } \\
\text { reduction in inflammation } \\
\text { for the intervention "No sig- } \\
\text { nificance differences were } \\
\text { found" for PBI between the } 2 \\
\text { groups, p. } 68 \text {. }\end{array}$ \\
\hline & $\begin{array}{l}\text { Christen } \\
2007\end{array}$ & $\begin{array}{l}\text { Den- } \\
\text { tacare-Son- } \\
\text { odont elec- }\end{array}$ & $\begin{array}{l}\text { PBI (scale } \\
0-4)\end{array}$ & $\begin{array}{l}\text { Pooled me- } \\
\text { dian } 12.3\end{array}$ & $\begin{array}{l}\text { Pooled me- } \\
\text { dian } 12.6\end{array}$ & 72 & Germany & $\begin{array}{l}\text { Cross-over trial. Da- } \\
\text { ta presented for the } \\
2 \text { arms combined. }\end{array}$ & $\begin{array}{l}\text { No strong evidence of a differ- } \\
\text { ence in the PBI median at the } \\
\text { final examination and at the }\end{array}$ \\
\hline
\end{tabular}


Table 8. Electric versus manual toothbrushes (RCTs): gingival inflammation short term (Continued)

$\begin{array}{lll}\text { tric TB vs } & \text { Self } \mathrm{Br} 9.5 & \text { Self } \mathrm{Br} 10.0 \\ \text { Oral B man- } & \text { Assist } \mathrm{Br} & \text { Assist } \mathrm{Br} \\ \text { ual TB } & 13.5 \text { Other } & 12.5 \text { Other } \\ & \mathrm{Br} 14.0 & \mathrm{Br} 15.5\end{array}$

Pooled median data calculated from reported data. initial examination (Friedman test: $P>0.05)$, p. 62

$\mathrm{Br} 14.0$

Pooled mean calcu- Both brushes seemed equally

lated from reported

data, no SD provid ed.

effective "None of the other

factors examined (except fre-

quency) produced an $\mathrm{F}$ values

approaching the $5 \%$ level of

al 2.08; fe- al 2.34; fe-

significance," p. 377.

live manual $\quad$ cal $0.14, \quad$ cal 2.63

TB

labial $0.15 \quad$ labial 2.65

\begin{tabular}{|c|c|c|c|c|c|c|c|c|c|}
\hline 4 weeks & Shaw 1983 & $\begin{array}{l}\text { Braun elec- } \\
\text { tric TB vs } \\
\text { Oral B } 30 \\
\text { manual TB }\end{array}$ & $\begin{array}{l}\text { WHOGI } \\
\text { (Scale } 0 \text { - } \\
\text { 3) }\end{array}$ & $1.7(1.9)$ & $1.6(1.7)$ & 106 & UK & $\begin{array}{l}\text { Cross-over trial. Data } \\
\text { presented for the } 2 \\
\text { arms combined. }\end{array}$ & $\begin{array}{l}\text { No strong evidence of a dif- } \\
\text { ference in oral hygiene per- } \\
\text { formance when comparing } \\
\text { the use of electric and man- } \\
\text { ual TBs. "There were no dif- } \\
\text { ferences between the two } \\
\text { groups. (t=0.0702, d.f. =/-52, p } \\
>0.05 \text { )," p. } 5 \text {. }\end{array}$ \\
\hline
\end{tabular}

Assist Br: assisted brusher; B\&LL\&SGI: Buccal and Labial surfaces Löe \& Silness: scale 0-3; Other Br: other brusher; PBI: Papillary Bleeding Index: scale 0-4; RCT: randomised controlled trial; SD: standard deviation; Self Br: self-brusher; TB: toothbrush; WHOGI: World Health Organization Technical Report No 621 Gingival Index: scale 0-3.

Christen 2007: three-armed cross-over trial; also compared the Superbrush, reported separately. Median and percentile data only reported. Data presented for Self Br, Assist Br and Other Br; pooled data were calculated from these data. Data for buccal and lingual surfaces, maxilla and mandible, also reported. Kaschke 2005: 3-armed cross-over trial; also compared the Superbrush, reported separately. Data for buccal and lingual surfaces, maxilla and mandible and Self Br, Assist Br and Other Br also reported. Swallow 1969: means presented separately for labial and buccal surfaces for males and females with no SD; the pooled means were calculated from these data. Female data at 21 days appeared skewed. Study also compared brushing once a week and twice a week, reported separately.

Table 9. Electric versus manual toothbrushes (RCTs): gingival inflammation medium term

\begin{tabular}{|c|c|c|c|c|c|c|c|c|c|}
\hline Duration & Study & Comparison & Index & $\begin{array}{l}\text { Interven- } \\
\text { tion mean } \\
\text { (SD) }\end{array}$ & $\begin{array}{l}\text { Control } \\
\text { mean (SD) }\end{array}$ & Total & Country & Comments & Summary \\
\hline 3 months & Carr 1997 & $\begin{array}{l}\text { Interplak elec- } \\
\text { tric TB vs Oral B } \\
40 \text { manual TB }\end{array}$ & $\begin{array}{l}\text { L\&SGI } \\
\text { (scale 0-3) }\end{array}$ & $\begin{array}{l}\text { Pooled } \\
\mathbf{1 . 8 0}(\mathbf{0 . 3 2}) \\
\text { Self } \mathrm{Br} 1.81 \\
(0.31) \mathrm{As}- \\
\text { sist Br } 1.78 \\
(0.33)\end{array}$ & $\begin{array}{l}\text { Pooled } \\
\mathbf{1 . 8 4}(\mathbf{0 . 2 5}) \\
\text { Self } \mathrm{Br} 1.81 \\
(0.28) \mathrm{As}- \\
\text { sist } \mathrm{Br} 1.90 \\
(0.21)\end{array}$ & 56 & USA & $\begin{array}{l}\text { Pooled mean and } \\
\text { SD calculated } \\
\text { from the reported } \\
\text { data. Cluster RCT. }\end{array}$ & $\begin{array}{l}\text { No strong evidence of a dif- } \\
\text { ference in gingival indices } \\
\text { between toothbrushes at } 3 \\
\text { months. }\end{array}$ \\
\hline
\end{tabular}


Table 9. Electric versus manual toothbrushes (RCTs): gingival inflammation medium term (Continued)

\begin{tabular}{|c|c|c|c|c|c|c|c|c|c|}
\hline & $\begin{array}{l}\text { Gar- } \\
\text { cia-Carril- } \\
\text { lo } 2016\end{array}$ & $\begin{array}{l}\text { Sonicare Easy- } \\
\text { Clean, Philips } \\
\text { electric TB vs } \\
\text { Vitis Access, } \\
\text { Dentaid manual } \\
\text { TB }\end{array}$ & $\begin{array}{l}\text { BDL\&SGI } \\
\text { (scale 0-3) }\end{array}$ & $1.31(0.32)$ & $1.24(0.32)$ & 64 & Spain & $\begin{array}{l}\text { Description of ID } \\
\text { in this study ap- } \\
\text { peared to include } \\
\text { some people with } \\
\text { an IQ > 70. }\end{array}$ & $\begin{array}{l}\text { No strong evidence of a dif- } \\
\text { ference in gingival indices } \\
\text { between toothbrushes at } 3 \\
\text { months. }\end{array}$ \\
\hline 4 months & $\begin{array}{l}\text { Kelner } \\
1963\end{array}$ & $\begin{array}{l}\text { Automated } \\
\text { electric TB } \\
\text { (Broxodent) vs } \\
\text { conventional } \\
\text { TB }\end{array}$ & SGR & $\begin{array}{l}\text { \% Improve- } \\
\text { ment 53\% }\end{array}$ & $\begin{array}{l}\text { \% Improve- } \\
\text { ment } 31 \%\end{array}$ & 100 & USA & $\begin{array}{l}\text { \% Improvement } \\
\text { calculated from } \\
\text { the reported da- } \\
\text { ta. }\end{array}$ & $\begin{array}{l}\text { "The automatic electric } \\
\text { toothbrush appeared signifi- } \\
\text { cantly superior to the conven- } \\
\text { tional toothbrush in improv- } \\
\text { ing gingival health," p. } 108 \text {. }\end{array}$ \\
\hline \multirow[t]{2}{*}{6 months } & Carr 1997 & $\begin{array}{l}\text { Interplak elec- } \\
\text { tric TB vs Oral B } \\
40 \text { manual TB }\end{array}$ & $\begin{array}{l}\text { L\&SGI } \\
\text { (scale 0-3) }\end{array}$ & $\begin{array}{l}\text { Pooled } \\
\mathbf{1 . 9 0}(0.20) \\
\text { Self } \mathrm{Br} 1.92 \\
(0.16) \text { As- } \\
\text { sist } \mathrm{Br} 1.86 \\
(0.23)\end{array}$ & $\begin{array}{l}\text { Pooled } \\
\mathbf{1 . 9 0}(\mathbf{0 . 1 6}) \\
\text { Self Br } 1.88 \\
(0.20) \text { As- } \\
\text { sist Br } 1.96 \\
(0.12)\end{array}$ & 56 & USA & $\begin{array}{l}\text { Pooled mean and } \\
\text { SD calculated } \\
\text { from the reported } \\
\text { data. Cluster RCT. }\end{array}$ & $\begin{array}{l}\text { No strong evidence of a dif- } \\
\text { ference in gingival indices } \\
\text { between toothbrushes at } 6 \\
\text { months. }\end{array}$ \\
\hline & $\begin{array}{l}\text { Gar- } \\
\text { cia-Carril- } \\
\text { lo } 2016\end{array}$ & $\begin{array}{l}\text { Sonicare Easy- } \\
\text { Clean, Philips } \\
\text { electric TB vs } \\
\text { Vitis Access, } \\
\text { Dentaid manual } \\
\text { TB }\end{array}$ & $\begin{array}{l}\text { BDL\&SGI } \\
\text { (scale 0-3) }\end{array}$ & $1.24(0.28)$ & $1.19(0.28)$ & 64 & Spain & $\begin{array}{l}\text { Description of ID } \\
\text { in this study ap- } \\
\text { peared to include } \\
\text { some people with } \\
\text { an IQ }>70 \text {. }\end{array}$ & $\begin{array}{l}\text { No strong evidence of a dif- } \\
\text { ference in gingival indices } \\
\text { between toothbrushes at } 6 \\
\text { months }(P=0.37) \text {, p. } 5 \text {. }\end{array}$ \\
\hline 12 months & Carr 1997 & $\begin{array}{l}\text { Interplak elec- } \\
\text { tric TB vs Oral B } \\
40 \text { manual TB }\end{array}$ & $\begin{array}{l}\text { L\&SGI } \\
\text { (scale 0-3) }\end{array}$ & $\begin{array}{l}\text { Pooled } \\
\mathbf{1 . 2 5}(\mathbf{0 . 4 7 )} \\
\text { Self } \mathrm{Br} 1.72 \\
(0.30) \text { As- } \\
\text { sist Br } 1.72 \\
(0.42)\end{array}$ & $\begin{array}{l}\text { Pooled } \\
\mathbf{1 . 7 3}(\mathbf{0 . 6 1}) \\
\text { Self Br } 1.87 \\
(0.21) \text { Assist } \\
\mathrm{Br} 2.0(0.0)\end{array}$ & 56 & USA & $\begin{array}{l}\text { Pooled mean and } \\
\text { SD calculated } \\
\text { from the reported } \\
\text { data. Cluster RCT. }\end{array}$ & $\begin{array}{l}\text { RM analysis of variance } \\
\text { showed the Gingival Index at } \\
12 \text { months was significant- } \\
\text { ly associated with the tooth- } \\
\text { brush used }(P=0.017) \text { posi- } \\
\text { tive for the Interplak electric } \\
\text { TB. }\end{array}$ \\
\hline
\end{tabular}

Assist Br: assisted brusher; BDL\&SGI: Bentley \& Disney (four sites per tooth in two randomly (by coin toss) selected quadrants (one in the upper jaw, one in the lower jaw, contralateral); IQ: intelligence quotient; L\&SGI: Löe \& Silness Gingival Index, Scale 0-3; Other Br: other brusher; RCT: randomised controlled trial; RM: repeat measure; SD: standard deviation; Self Br: self-brusher; SGR: Subjective Gingival Rating Scale, - worse, same, better; TB: toothbrush.

Kelner 1963: subjective data presented. Data also presented for frequency of brushing. Carr 1997: means and SDs presented separately for Self Br and Assist Br; the pooled means and SDs were calculated from these data for the different time points. 
Table 10. Electric versus manual toothbrushes (NRS): gingival inflammation short term

\begin{tabular}{|c|c|c|c|c|c|c|c|c|c|}
\hline Duration & Study & Comparison & Index & $\begin{array}{l}\text { Intervention mean } \\
\text { (SD) }\end{array}$ & $\begin{array}{l}\text { Control mean } \\
\text { (SD) }\end{array}$ & Total & Country & Comments & Summary \\
\hline 4 weeks & $\begin{array}{l}\text { Bratel } \\
1991\end{array}$ & $\begin{array}{l}\text { Braun 3D Elec- } \\
\text { tric toothbrush vs } \\
\text { Butler } 411 \text { manu- } \\
\text { al toothbrush. }\end{array}$ & $\begin{array}{l}\text { L\&SGI } \\
\text { (scale 0-3) }\end{array}$ & $\begin{array}{l}\text { Pooled } 1.55(\mathbf{0 . 1 6 )} \\
\text { Unaided } 1.5(0.2), \\
\text { aided } 1.6(0.1)\end{array}$ & $\begin{array}{l}\text { Pooled } \\
\mathbf{1 . 6 0}(\mathbf{0 . 1 6}) \text { Unaid- } \\
\text { ed } 1.6(0.1) \text {, aided } \\
1.6(0.2)\end{array}$ & 23 & Sweden & $\begin{array}{l}\text { Pooled mean } \\
\text { and SD calcu- } \\
\text { lated from the } \\
\text { reported data. }\end{array}$ & $\begin{array}{l}\text { No overall dif- } \\
\text { ference in Gin- } \\
\text { gival Index be- } \\
\text { tween tooth- } \\
\text { brushes. }\end{array}$ \\
\hline
\end{tabular}

L\&SGI: Löe \& Silness Gingival Index, Scale 0-3; NRS: non-randomised study; SD: standard deviation.

Bratel 1991: mean and SD presented for aided and unaided brushing, pooled mean and SDs were calculated from these data.

\section{Table 11. Electric versus manual toothbrushes (NRS): gingival inflammation medium term}

\begin{tabular}{|c|c|c|c|c|c|c|c|c|c|}
\hline Duration & Study & Comparison & Index & $\begin{array}{l}\text { Interven- } \\
\text { tion mean } \\
\text { (SD) }\end{array}$ & $\begin{array}{l}\text { Control } \\
\text { mean (SD) }\end{array}$ & Total & Country & Comments & Summary \\
\hline \multirow[t]{2}{*}{8 weeks } & $\begin{array}{l}\text { Gertenrich } \\
1967 c\end{array}$ & $\begin{array}{l}\text { Oral B Automatic } \\
\text { Toothbrush - Arcu- } \\
\text { ate actions vs man- } \\
\text { ual brush (Py-co- } \\
\text { pay, Hard, Lactona } \\
\text { Jr.) }\end{array}$ & $\begin{array}{l}\text { SGR (scale } \\
1-3)\end{array}$ & $\begin{array}{l}\text { Pooled } \\
\mathbf{3 4 \%} \\
\text { Group } \\
\text { 1: } 10 \% ; \\
\text { Group 2: } \\
58 \%\end{array}$ & $\begin{array}{l}\text { Pooled } \\
\text { 43\% } \\
\text { Group } \\
\text { 1: } 50 \% \text {; } \\
\text { Group 2: } \\
\text { 36\% }\end{array}$ & 37 & USA & $\begin{array}{l}\text { Data presented as } \\
\text { mean level of "obvi- } \\
\text { ous gingivitis" by } \% \\
\text { in a graph, pooled } \% \\
\text { for the reported data } \\
\text { were calculated. }\end{array}$ & $\begin{array}{l}\text { General reduction in gin- } \\
\text { givitis in both groups and } \\
\text { with both brushes. The } \\
\text { greatest reduction was in } \\
\text { Group } 1 \text { using the electric } \\
\text { brush. }\end{array}$ \\
\hline & $\begin{array}{l}\text { Gertenrich } \\
1967 a\end{array}$ & $\begin{array}{l}\text { Oral B Automatic } \\
\text { Toothbrush - Arcu- } \\
\text { ate actions vs man- } \\
\text { ual brush (Py-co- } \\
\text { pay, Hard, Lactona } \\
\text { Jr.) }\end{array}$ & $\begin{array}{l}\text { SGR (scale } \\
1-3)\end{array}$ & $\begin{array}{l}\text { Pooled } \\
\mathbf{8 3 \%} \\
\text { Group } \\
\text { 1: 74\%; } \\
\text { Group } \\
\text { 2a: 85\%; } \\
\text { Group 2b: } \\
\text { 100\% }\end{array}$ & $\begin{array}{l}\text { Pooled } \\
\mathbf{5 4 \%} \\
\text { Group } \\
\text { 1: 50\%; } \\
\text { Group } \\
\text { 2c: } 50 \% ; \\
\text { Group 2d:; } \\
65 \%\end{array}$ & 38 & USA & $\begin{array}{l}\text { Data presented as } \\
\text { mean level of "obvi- } \\
\text { ous gingivitis" by } \% \\
\text { in a graph, Pooled \% } \\
\text { for the reported data } \\
\text { were calculated. }\end{array}$ & $\begin{array}{l}\text { There was a general re- } \\
\text { duction in both groups } \\
\text { and both brushes, with a } \\
\text { tendency for greater re- } \\
\text { duction in those using a } \\
\text { standard brush. }\end{array}$ \\
\hline $\begin{array}{l}8 / 10 \\
\text { weeks }\end{array}$ & $\begin{array}{l}\text { Gertenrich } \\
1967 b\end{array}$ & $\begin{array}{l}\text { Oral B Automatic } \\
\text { Toothbrush - Arcu- }\end{array}$ & $\begin{array}{l}\text { SGR (scale } \\
1-3)\end{array}$ & $\begin{array}{l}\text { Pooled } \\
57.5 \%\end{array}$ & $\begin{array}{l}\text { Pooled } \\
77 \%\end{array}$ & 70 & USA & $\begin{array}{l}\text { Data presented as } \\
\text { mean level of "obvi- }\end{array}$ & $\begin{array}{l}\text { General reduction in gin- } \\
\text { givitis with both brush- }\end{array}$ \\
\hline
\end{tabular}


Table 11. Electric versus manual toothbrushes (NRS): gingival inflammation medium term (Continued)

\begin{tabular}{|c|c|c|c|c|c|c|c|}
\hline & & $\begin{array}{l}\text { ate actions vs man- } \\
\text { ual brush (Py-co- } \\
\text { pay, Hard, Lactona } \\
\text { Jr.) }\end{array}$ & & $\begin{array}{l}\text { Group } \\
\text { 1: 45\%; } \\
\text { Group 2: } \\
70 \%\end{array}$ & $\begin{array}{l}\text { Group } \\
\text { 1: 54\%; } \\
\text { Group 2: } \\
100 \%\end{array}$ & & \\
\hline 12 weeks & $\begin{array}{l}\text { Bratel } \\
1991\end{array}$ & $\begin{array}{l}\text { Braun 3D Electric } \\
\text { toothbrush vs But- } \\
\text { ler } 411 \text { manual } \\
\text { toothbrush }\end{array}$ & $\begin{array}{l}\text { L\&SGI } \\
\text { (scale 0-3) }\end{array}$ & $\begin{array}{l}\text { Pooled } \\
\mathbf{1 . 6 5} \\
\mathbf{( 0 . 1 0 )} \\
\text { Group 1: } \\
1.6(0.1) ; \\
\text { Group 2: } \\
1.7(0.1)\end{array}$ & $\begin{array}{l}\text { Pooled } \\
\mathbf{1 . 6 0} \\
\mathbf{( 0 . 1 6 )} \\
\text { Group 1: } \\
1.6(0.2) ; \\
\text { Group 2: } \\
1.6(0.1)\end{array}$ & 23 & Sweden \\
\hline
\end{tabular}

ous gingivitis" by $\%$ in a graph, pooled $\%$ for the reported data were calculated.

Pooled mean and SD

calculated from the reported data. es. A greater reduction in Group 1 (participants with Down's syndrome) for both brushes.

No strong evidence of a difference in Gingival Index between the 2 brushes. Group 1 (unaided) using the electric TB brush was the only subgroup with strong evidence of improvement from baseline at 12 weeks $(P<0.01)$, p. 25.

\begin{tabular}{|c|c|c|c|c|c|c|c|c|c|}
\hline 20 weeks & $\begin{array}{l}\text { Gertenrich } \\
1967 a\end{array}$ & $\begin{array}{l}\text { Oral B Automatic } \\
\text { Toothbrush - Arcu- } \\
\text { ate actions vs man- } \\
\text { ual brush (Py-co- } \\
\text { pay, Hard, Lactona } \\
\text { Jr.) }\end{array}$ & $\begin{array}{l}\text { SGR (scale } \\
1-3)\end{array}$ & $\begin{array}{l}\text { Pooled } \\
\mathbf{1 0 0 \%} \\
\text { Group } \\
\text { 1: } 100 \% ; \\
\text { Group } \\
\text { 2a: } 100 \% ; \\
\text { Group 2b: } \\
100 \%\end{array}$ & $\begin{array}{l}\text { Pooled } \\
\mathbf{7 5 \%} \\
\text { Group } \\
\text { 1: 60\%; } \\
\text { Group } \\
\text { 2a: 85\%; } \\
\text { Group 2b: } \\
\text { 85\% }\end{array}$ & 36 & USA & $\begin{array}{l}\text { Data presented as } \\
\text { mean level of "obvi- } \\
\text { ous gingivitis" by } \% \\
\text { in a graph, Pooled } \% \\
\text { for the reported data } \\
\text { were calculated. }\end{array}$ & $\begin{array}{l}\text { Levels of observable gin- } \\
\text { givitis regressed towards } \\
\text { baseline levels, with } \\
\text { slightly less regression in } \\
\text { the those using the stan- } \\
\text { dard toothbrush. }\end{array}$ \\
\hline $\begin{array}{l}21 / 28 \\
\text { weeks }\end{array}$ & $\begin{array}{l}\text { Gertenrich } \\
\text { 1967b }\end{array}$ & $\begin{array}{l}\text { Oral B Automatic } \\
\text { Toothbrush - Arcu- } \\
\text { ate actions vs man- } \\
\text { ual brush (Py-co- } \\
\text { pay, Hard, Lactona } \\
\text { Jr.) }\end{array}$ & $\begin{array}{l}\text { SGR (scale } \\
1-3)\end{array}$ & $\begin{array}{l}\text { Pooled } \\
\mathbf{5 3 . 5 \%} \\
\text { Group } \\
\text { 1:37\%; } \\
\text { Group 2: } \\
\text { 70\% }\end{array}$ & $\begin{array}{l}\text { Pooled } \\
\mathbf{8 5 \%} \\
\text { Group } \\
\text { 1: } 70 \% \text {; } \\
\text { Group 2: } \\
\text { 100\% }\end{array}$ & 72 & USA & $\begin{array}{l}\text { Data presented as } \\
\text { mean level of "obvi- } \\
\text { ous gingivitis" by } \% \\
\text { in a graph, Pooled } \% \\
\text { for the reported data } \\
\text { were calculated. }\end{array}$ & $\begin{array}{l}\text { The reduction in obvi- } \\
\text { ous gingivitis seen at } 11 \\
\text { weeks was maintained for } \\
\text { both Group } 1 \text { (Down's syn- } \\
\text { drome) and Group } 2 \text { (IQ < } \\
\text { 20) using the electric TB } \\
\text { at this time point. Both } \\
\text { groups using the manual } \\
\text { TB regressed to baseline } \\
\text { levels. }\end{array}$ \\
\hline 34 weeks & $\begin{array}{l}\text { Gertenrich } \\
1967 c\end{array}$ & $\begin{array}{l}\text { Oral B Automatic } \\
\text { Toothbrush - Arcu- }\end{array}$ & $\begin{array}{l}\text { SGR (scale } \\
1-3)\end{array}$ & $\begin{array}{l}\text { Pooled } \\
42.5 \%\end{array}$ & $\begin{array}{l}\text { Pooled } \\
40 \%\end{array}$ & 36 & USA & $\begin{array}{l}\text { Data presented as } \\
\text { mean level of "obvi- }\end{array}$ & $\begin{array}{l}\text { Some reduction in gingivi- } \\
\text { tis was maintained from }\end{array}$ \\
\hline
\end{tabular}


Table 11. Electric versus manual toothbrushes (NRS): gingival inflammation medium term (Continued)

$\begin{array}{lll}\text { ate actions vs man- } & \text { Group } & \text { Group } \\ \text { ual brush (Py-co- } & 1: 40 \% ; & 1: 30 \% ; \\ \text { pay, Hard, Lactona } & \text { Group 2: } & \text { Group 2: } \\ \text { Jr.) } & 45 \% & 50 \%\end{array}$

L\&SGI: Löe \& Silness Gingival Index, Scale 0-3; NRS: non-randomised study; SD: standard deviation; SGR: Subjective Gingival Rating, measure of "periodontal involvement;" "obvious signs of gingivitis;" "decrease in severity" and "absence of clinical signs of gingivitis." "Obvious signs" and "decrease in severity" were combined on the graph. IQ: intelligence quotient; TB: toothbrush.

Group 1: unaided; Group 2: aided.

Gertenrich 1967b: data presented as \% difference separately for two distinct ID groups (Group 1: Down's syndrome; Group 2: intelligence quotient (IQ): $\leq 20)$ the pooled \% differences were calculated from these data. All teeth were brushed by attendants. Gertenrich 1967c: data presented as \% difference separately for two distinct groups with ID

(Group 1: hydrocephaly and seizure unit; Group 2: paediatric unit); the pooled \% differences were calculated from these data. All teeth were brushed by attendants. Gertenrich 1967a: in Group 1 (people with cerebral palsy with IQ 30-95), all teeth were brushed by attendants. In Group 2 ("trainable patients" with IQ 30-50), teeth of half the participants were brushed by attendants ( $2 \mathrm{a}$ and $2 \mathrm{c}$ ), the other half self-brushed ( $2 \mathrm{~b}$ and $2 \mathrm{~d})$. Gertenrich $1967 \mathrm{~d}$ : all participants were self-brushers with supervision and assistance from the attendants. Bratel 1991: mean and SD presented for unaided brushing (Group 1), aided brushing (Group 2), and pooled mean and SDs were calculated from these data. Wear and tear on toothbrushes was reported as having no difference, no data provided. Participants in the intervention group preferred the electric toothbrush, no data provided for the control group.

\section{Table 12. Electric versus manual toothbrushes (NRS): gingival inflammation long term}

\begin{tabular}{|c|c|c|c|c|c|c|c|c|c|}
\hline Duration & Study & $\begin{array}{l}\text { Compari- } \\
\text { son }\end{array}$ & Index & $\begin{array}{l}\text { Interven- } \\
\text { tion mean } \\
\text { (SD) }\end{array}$ & $\begin{array}{l}\text { Control } \\
\text { mean (SD) }\end{array}$ & Total & Country & Comments & Summary \\
\hline 16 months & $\begin{array}{l}\text { Bratel } \\
1991\end{array}$ & $\begin{array}{l}\text { Braun 3D } \\
\text { Electric } \\
\text { toothbrush } \\
\text { vs Butler } \\
411 \text { manual } \\
\text { toothbrush }\end{array}$ & $\begin{array}{l}\text { L\&SGI } \\
\text { (scale 0-3) }\end{array}$ & $\begin{array}{l}\text { Pooled } \mathbf{1 . 7 5} \\
\mathbf{( 0 . 1 6 )} \\
\text { Group 1: } 1.7 \\
(0.1) ; \text { Group } \\
\text { 2: } 1.8(0.2)\end{array}$ & $\begin{array}{l}\text { Pooled } 1.70 \\
(\mathbf{0 . 2 0 )} \\
\text { Group 1: } 1.7 \\
(0.2) ; \text { Group } \\
2: 1.7(0.2)\end{array}$ & 23 & Sweden & $\begin{array}{l}\text { Data presented } \\
\text { for aided and un- } \\
\text { aided with SD, } \\
\text { overall pooled } \\
\text { mean and SD cal- } \\
\text { culated from the } \\
\text { reported data. }\end{array}$ & $\begin{array}{l}\text { No strong evidence of a differ- } \\
\text { ence in gingivitis between the } \\
\text { brushes. The unaided electric } \\
\text { TB subgroup showed greater im- } \\
\text { provement at } 16 \text { months }(P< \\
0.05) \text {. The aided manual TB sub- } \\
\text { group showed greater deteriora- } \\
\text { tion at } 16 \text { months }(P<0.01) \text {. }\end{array}$ \\
\hline
\end{tabular}

Group 1: unaided; Group 2: aided; L\&SGI: Löe \& Silness Gingival Index, Scale 0-3; NRS: non-randomised study; SD: standard deviation; TB: toothbrush Bratel 1991: mean and SD presented for unaided (Group 1) and aided (Group 2) brushing; pooled mean and SDs were calculated from these data.

\section{Table 13. Electric versus manual toothbrushes (RCTs): plaque short term}

Duration

Study

Compari-

Index

Interven-

Control

Total

Country

Comments

Summary

(SD)

ڤ్ڤ 
Table 13. Electric versus manual toothbrushes (RCTs): plaque short term (Continued)

\begin{tabular}{|c|c|c|c|c|c|c|c|c|c|}
\hline 1 week & $\begin{array}{l}\text { Dôgan } \\
2004\end{array}$ & $\begin{array}{l}\text { Braun } \\
\text { Plaque Con- } \\
\text { trol 3D elec- } \\
\text { tric TB vs } \\
\text { Oral B Cross } \\
\text { Action 35/40 } \\
\text { manual TB }\end{array}$ & $\begin{array}{l}\text { MQHPI } \\
\text { (scale 0-5) }\end{array}$ & $1.54(0.71)$ & $2.15(0.58)$ & 15 & Turkey & $\begin{array}{l}\text { Cross-over trial. Da- } \\
\text { ta presented for the } \\
2 \text { arms separately at } \\
\text { the final examination. } \\
\text { Participant number at } \\
\text { baseline unclear: as- } \\
\text { sumed to be the same } \\
\text { as at final time point. }\end{array}$ & $\begin{array}{l}\text { Strong evidence of a differ- } \\
\text { ence in the Plaque Index be- } \\
\text { tween the Braun 3D electric TB } \\
\text { and Oral B Cross Action TB }(P< \\
0.001) \text { in favour of the electric } \\
\text { TB. }\end{array}$ \\
\hline \multirow[t]{2}{*}{2 weeks } & $\begin{array}{l}\text { Christen } \\
2007\end{array}$ & $\begin{array}{l}\text { Den- } \\
\text { tacare-Son- } \\
\text { odont elec- } \\
\text { tric TB vs } \\
\text { Oral B man- } \\
\text { ual TB }\end{array}$ & $\begin{array}{l}\text { MQHPI } \\
\text { (scale 0-5) }\end{array}$ & $\begin{array}{l}\text { Pooled } \\
\text { median } \\
\mathbf{2 5 . 8} \\
\text { Self } \mathrm{Br} \\
23.0 \mathrm{As}- \\
\text { sist } \mathrm{Br} 27.5 \\
\text { Other } \mathrm{Br} \\
27.0\end{array}$ & $\begin{array}{l}\text { Pooled } \\
\text { median } \\
\mathbf{2 8 . 3} \\
\text { Self Br } \\
26.0 \mathrm{As}- \\
\text { sist } \mathrm{Br} 28.0 \\
\text { Other } \mathrm{Br} \\
31.0\end{array}$ & 72 & Germany & $\begin{array}{l}\text { Cross-over trial. Da- } \\
\text { ta presented for the } \\
2 \text { arms combined. } \\
\text { Pooled median data } \\
\text { calculated from re- } \\
\text { ported data. }\end{array}$ & $\begin{array}{l}\text { No strong evidence of a differ- } \\
\text { ence in the QH median across } \\
\text { all participants between the } 3 \\
\text { different toothbrushes in the } \\
\text { final examination (Friedman } \\
\text { test: } P>0.05 \text { ), p. } 55 \text {. }\end{array}$ \\
\hline & $\begin{array}{l}\text { Kaschke } \\
2005\end{array}$ & $\begin{array}{l}\text { Teledyne } \\
\text { Waterpik } \\
\text { Sonic Speed } \\
\text { electric TB } \\
\text { vs Oral B } \\
\text { manual TB }\end{array}$ & $\begin{array}{l}\text { MQHPI } \\
\text { (scale 0-5) }\end{array}$ & $\begin{array}{l}\text { Whole } \\
\text { mouth } 3.1 \\
(0.09) \text { Sur- } \\
\text { face score } \\
36.1(1.2)\end{array}$ & $\begin{array}{l}\text { Whole } \\
\text { mouth } \\
\mathbf{3 . 2 5} \\
(0.10) \text { Sur- } \\
\text { face score } \\
38.9(1.9)\end{array}$ & 54 & Germany & $\begin{array}{l}\text { Cross-over trial. Da- } \\
\text { ta presented for the } 2 \\
\text { arms combined. Sur- } \\
\text { face score Mean and } \\
\text { SD obtained from au- } \\
\text { thor. Data calculat- } \\
\text { ed from tooth surface } \\
\text { scores. Median pre- } \\
\text { sented in published ar- } \\
\text { ticle. }\end{array}$ & $\begin{array}{l}\text { Strong evidence that the mean } \\
\text { Plaque Index for the Teledyne } \\
\text { Waterpik was lower than the } \\
\text { Oral B manual toothbrush }(P< \\
0.05), \text { p. } 68 \text {. }\end{array}$ \\
\hline 4 weeks & Shaw 1983 & $\begin{array}{l}\text { Braun elec- } \\
\text { tric TB vs } \\
\text { Oral B } 30 \\
\text { manual TB }\end{array}$ & $\begin{array}{l}\text { S\&LPI } \\
\text { (scale 0-3) }\end{array}$ & $\begin{array}{l}\text { Total } \\
\text { mouth } \\
\mathbf{0 . 5 9} \\
(0.26) \text { Sur- } \\
\text { face score } \\
14.1(6.2)\end{array}$ & $\begin{array}{l}\text { Total } \\
\text { mouth } \\
\mathbf{0 . 5 7}(\mathbf{0 . 2 )} \\
\text { Surface } \\
\text { score } 13.6 \\
(4.7)\end{array}$ & 106 & UK & $\begin{array}{l}\text { Cross-over trial. Da- } \\
\text { ta presented for the } 2 \\
\text { arms combined. Data } \\
\text { calculated from tooth } \\
\text { surface scores. }\end{array}$ & $\begin{array}{l}\text { No strong evidence of a differ- } \\
\text { ence in oral hygiene perfor- } \\
\text { mance when comparing the } \\
\text { use of electric and manual TBs. } \\
\text { "There was no sig. diff between } \\
\text { the groups ( } t=0.833 \text {, d.f.52, } p> \\
0.05 \text { )," p. } 5 \text {. }\end{array}$ \\
\hline
\end{tabular}

Assist Br: assisted brusher; MQHPI: Modified Quigley-Hein Plaque Index, scale 0-5; Other Br: other brusher; QH: Quigley-Hein; RCT: randomised controlled trial; S\&LPI: Silness \& Löe Plaque Index, scale 0-3; SD: standard deviation; Self Br: self-brusher; TB: toothbrush.

Dôgan 2004: three-armed cross-over trial; also compared the Superbrush, reported separately. Data presented were for the final examination, number of participants were adjusted accordingly. Data for age groups and surfaces also reported. Christen 2007: three-armed cross-over trial; also compared the Superbrush, reported separately. Median and percentile data only reported. Data presented for Self $\mathrm{Br}$, Assist $\mathrm{Br}$ and Other $\mathrm{Br}$, pooled data were calculated from these data. Data for buccal and lingual surfaces, maxilla and mandible also reported. Kaschke 2005: three-armed cross-over trial; also compared the Superbrush, reported separately. Data for buccal and lingual surfaces, maxilla and 


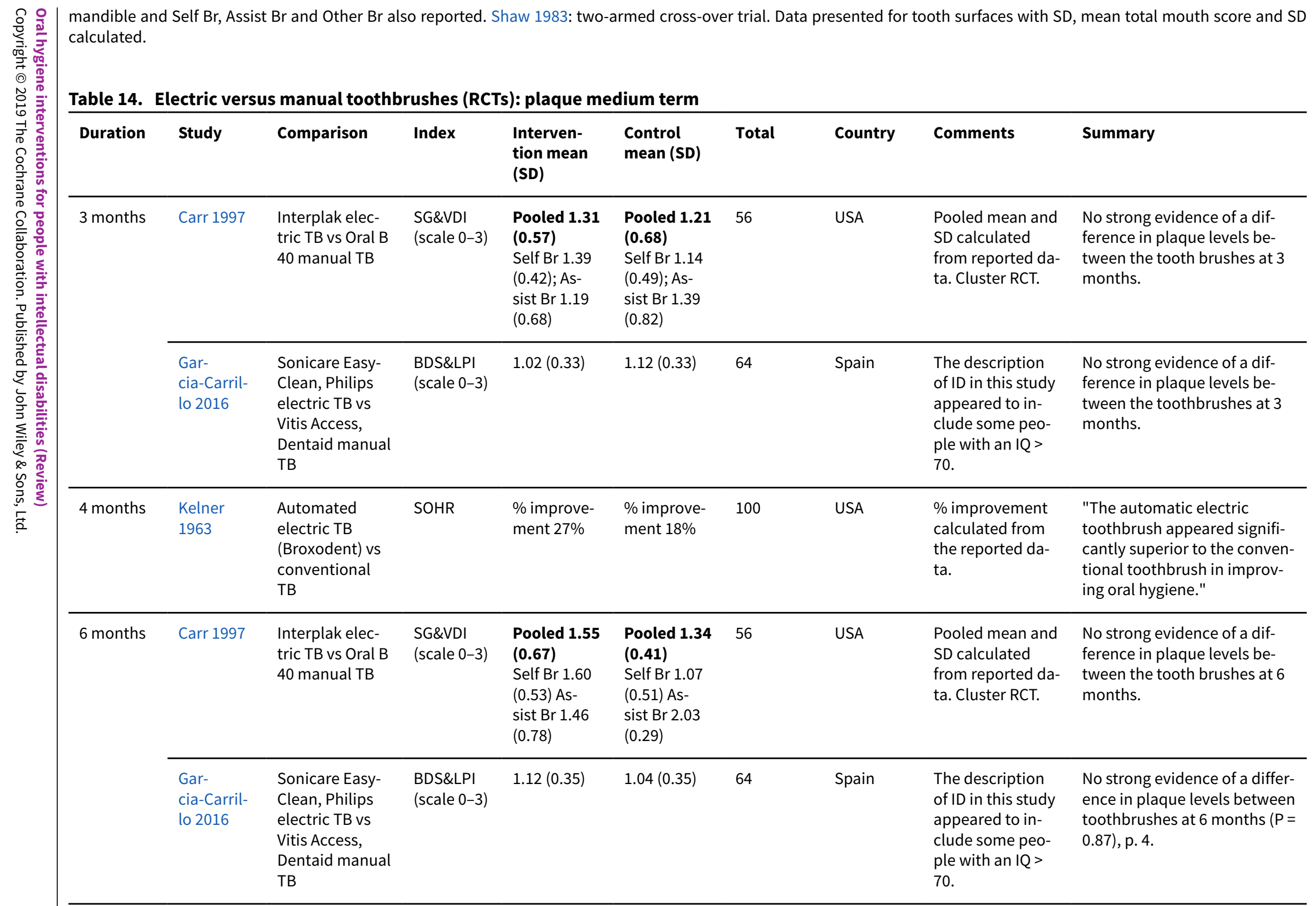


Table 14. Electric versus manual toothbrushes (RCTs): plaque medium term (Continued)

12 months Carr 1997

$\begin{array}{ll}\text { Interplak elec- } & \text { SG\&VDI } \\ \text { tric TB vs Oral B } & \text { (scale 0-3) } \\ 40 \text { manual TB } & \end{array}$

Pooled 1.35 Pooled 1.4956

(0.57)

Self $\mathrm{Br} 1.25$

(0.51); As-

sist $\mathrm{Br} 1.15$

(0.63)
(0.51)

Self $\mathrm{Br} 1.25$

(0.42); As-

sist $\mathrm{Br} 2.10$

Assist Br: assisted brusher; BDS\&LPI: Bentley \& Disney Modified Silness \& Löe Plaque Index, scale 0-3; ID: intellectual disability; IQ: intelligence quotient; RCT: randomised controlled trial; SD: standard deviation; Self Br: self-brusher; SG\&VDI: Simplified Green \& Vermillion Debris Index, scale 0-3; SOHR: Subjective Oral Hygiene Rating, scale poor, fair, good; TB: toothbrush.

Kelner 1963: subjective data presented, \% improvement has been calculated from the differences between the combined fair and good ratings pre and post. Data also presented by age groups (aged 4-14 years, 15-32 years). Carr 1997: means and SDs presented separately for Self Br and Assist Br, the pooled means and SDs were calculated from these data for the different time points. Some differences noted between Self $\mathrm{Br}$ and Assist $\mathrm{Br}$. "There was a marginally significant difference in the pattern of debris index across time for the two brushing assistance status groups $(p=0.054), "$ p. 135. Garcia-Carrillo 2016 reported the mean IQ as 60.6 , ranging between 44 and 87.

\section{Table 15. Electric versus manual toothbrushes (NRS): plaque short term}

\begin{tabular}{|c|c|c|c|c|c|c|c|c|c|}
\hline Duration & Study & Comparison & Index & $\begin{array}{l}\text { Intervention mean } \\
\text { (SD) }\end{array}$ & $\begin{array}{l}\text { Control mean } \\
\text { (SD) }\end{array}$ & Total & Country & Comments & Summary \\
\hline 4 weeks & $\begin{array}{l}\text { Bratel } \\
1991\end{array}$ & $\begin{array}{l}\text { Braun 3D Elec- } \\
\text { tric toothbrush vs } \\
\text { Butler } 411 \text { manu- } \\
\text { al toothbrush }\end{array}$ & $\begin{array}{l}\text { S\&LPI } \\
\text { (scale 0-3) }\end{array}$ & $\begin{array}{l}\text { Pooled } 1.5(0.3) \text { Un- } \\
\text { aided } 0.8(0.3) ; \text { aided } \\
1.3(0.5)\end{array}$ & $\begin{array}{l}\text { Pooled } 1.5(\mathbf{0 . 3 5}) \\
\text { Unaided } 1.1(0.4) \\
\text { aided } 1.0(0.3)\end{array}$ & 23 & Sweden & $\begin{array}{l}\text { Pooled mean } \\
\text { and SD calcu- } \\
\text { lated from the } \\
\text { reported data. }\end{array}$ & $\begin{array}{l}\text { No overall } \\
\text { difference in } \\
\text { Plaque Index } \\
\text { between tooth- } \\
\text { brushes. }\end{array}$ \\
\hline
\end{tabular}

NRS: non-randomised study; S\&LPI: Silness \& Löe Plaque Index, Scale 0-3; SD: standard deviation.

Bratel 1991: mean and SD presented for aided and unaided brushing, pooled mean and SDs were calculated from these data.

\section{Table 16. Electric versus manual toothbrushes (NRS): plaque medium term}

\begin{tabular}{|c|c|c|c|c|c|c|c|c|c|}
\hline Duration & Study & Comparison & Index & $\begin{array}{l}\text { Interven- } \\
\text { tion mean } \\
\text { (SD) }\end{array}$ & $\begin{array}{l}\text { Control } \\
\text { mean (SD) }\end{array}$ & Total & Country & Comments & Summary \\
\hline $\begin{array}{l}0-8 / 10 \\
\text { weeks }\end{array}$ & $\begin{array}{l}\text { Gertenrich } \\
1967 b\end{array}$ & $\begin{array}{l}\text { Oral B Automat- } \\
\text { ic Toothbrush - } \\
\text { Arcuate actions } \\
\text { vs manual brush } \\
\text { (Py-co-pay, Hard, }\end{array}$ & $\begin{array}{l}\text { SOHR } \\
\text { (scale 1-4) }\end{array}$ & $\begin{array}{l}\text { Pooled } \\
\text { MD 0.75 } \\
\text { (0.64) } \\
\text { Group 1: } \\
0.63(0.71) ;\end{array}$ & $\begin{array}{l}\text { Pooled } \\
\text { MD 0.59 } \\
(\mathbf{0 . 9 1 )} \\
\text { Group 1: } \\
0.32(1.08)\end{array}$ & 55 & USA & $\begin{array}{l}\text { Pooled mean dif- } \\
\text { ference and SD } \\
\text { calculated from } \\
\text { the reported da- } \\
\text { ta. }\end{array}$ & $\begin{array}{l}\text { The results showed that both } \\
\text { groups reduced plaque levels } \\
\text { during the supervised brushing } \\
\text { period, with the electric TB be- } \\
\text { ing "somewhat better." }\end{array}$ \\
\hline
\end{tabular}

$\begin{array}{ll}\text { Pooled mean and } & \text { No reported strong evidence } \\ \text { SD calculated } & \text { of a difference in plaque lev }\end{array}$

from reported da- els between tooth brushes at

ta. Cluster RCT. 12 months. ing "somewhat better." 
Table 16. Electric versus manual toothbrushes (NRS): plaque medium term (Continued)

Group 2: Group 2:

$1.18(0.56) \quad 1.12(0.70)$

\begin{tabular}{|c|c|c|c|c|c|c|c|c|c|}
\hline $0-8$ weeks & $\begin{array}{l}\text { Gertenrich } \\
1967 c\end{array}$ & $\begin{array}{l}\text { Oral B Automat- } \\
\text { ic Toothbrush - } \\
\text { Arcuate actions } \\
\text { vs manual brush } \\
\text { (Py-co-pay, Hard, } \\
\text { Lactona Jr.) }\end{array}$ & $\begin{array}{l}\text { SOHR } \\
\text { (scale 1-4) }\end{array}$ & $\begin{array}{l}\text { Pooled } \\
\text { MD 1.26 } \\
(\mathbf{0 . 5 0 )} \\
\text { Group 1: } \\
1.70(0.47) ; \\
\text { Group 2: } \\
0.66(0.53)\end{array}$ & $\begin{array}{l}\text { Pooled } \\
\text { MD 1.45 } \\
(\mathbf{0 . 7 3 )} \\
\text { Group 1: } \\
1.57(0.68) ; \\
\text { Group 2: } \\
1.25(0.77)\end{array}$ & 37 & USA & $\begin{array}{l}\text { Pooled MD and } \\
\text { SD calculated } \\
\text { from the reported } \\
\text { data. }\end{array}$ & $\begin{array}{l}\text { An improvement in oral hy- } \\
\text { giene in both ID groups and } \\
\text { with both brushes (P } 0.05- \\
0.001 \text { ). No strong evidence of } \\
\text { a difference in oral hygiene be- } \\
\text { tween the } 2 \text { brushes. }\end{array}$ \\
\hline $0-8$ weeks & $\begin{array}{l}\text { Gertenrich } \\
1967 a\end{array}$ & $\begin{array}{l}\text { Oral B Automat- } \\
\text { ic Toothbrush - } \\
\text { Arcuate actions } \\
\text { vs manual brush } \\
\text { (Py-co-pay, Hard, } \\
\text { Lactona Jr.) }\end{array}$ & $\begin{array}{l}\text { SOHR } \\
\text { (scale 1-4) }\end{array}$ & $\begin{array}{l}\text { Group } 1 \\
\text { MD 2.21 } \\
(0.60)\end{array}$ & $\begin{array}{l}\text { Group } 1 \\
\text { MD } 1.72 \\
(0.55)\end{array}$ & $\begin{array}{l}\text { Group 1: } \\
15\end{array}$ & USA & $\begin{array}{l}\text { MD and SD re- } \\
\text { ported for Group } \\
1 . \text { Mean square } \\
\text { difference was re- } \\
\text { ported for Group } \\
\text { 2. Type of brush- } \\
\text { mean Sq (F) } 2.67 \\
\text { (10.35). Author } \\
\text { reports raw data } \\
\text { no longer avail- } \\
\text { able. }\end{array}$ & $\begin{array}{l}\text { A reduction in plaque levels in } \\
\text { both ID groups and with both } \\
\text { brushes (P } 0.05-0.001 \text { ). No } \\
\text { strong evidence of a difference } \\
\text { between the } 2 \text { brushes. }\end{array}$ \\
\hline 12 weeks & $\begin{array}{l}\text { Bratel } \\
1991\end{array}$ & $\begin{array}{l}\text { Braun 3D Elec- } \\
\text { tric toothbrush vs } \\
\text { Butler } 411 \text { manu- } \\
\text { al toothbrush. }\end{array}$ & $\begin{array}{l}\text { S\&LPI } \\
\text { (scale 0-3) }\end{array}$ & $\begin{array}{l}\text { Pooled } \\
\mathbf{1 . 1 0} \\
(\mathbf{0 . 1 4}) \\
\text { Unaided } \\
0.9(0.3) \\
\text { aided } 1.3 \\
(0.2)\end{array}$ & $\begin{array}{l}\text { Pooled } \\
\mathbf{1 . 1 1} \\
\mathbf{( 0 . 3 0 )} \\
\text { Unaided } \\
1.2(0.3) \\
\text { aided } 1.0 \\
(0.3)\end{array}$ & 23 & Sweden & $\begin{array}{l}\text { Pooled mean and } \\
\text { SD calculated } \\
\text { from the reported } \\
\text { data. }\end{array}$ & $\begin{array}{l}\text { Some strong evidence of a dif- } \\
\text { ference in subgroups (manu- } \\
\text { al aided }(P<0.01) \text { and electric } \\
\text { unaided }(P<0.05) \text {; however, } \\
\text { no strong overall difference in } \\
\text { Plaque Index between tooth- } \\
\text { brushes. }\end{array}$ \\
\hline $\begin{array}{l}8-20 \\
\text { weeks }\end{array}$ & $\begin{array}{l}\text { Gertenrich } \\
1967 a\end{array}$ & $\begin{array}{l}\text { Oral B Automat- } \\
\text { ic Toothbrush - } \\
\text { Arcuate actions } \\
\text { vs manual brush } \\
\text { (Py-co-pay, Hard, } \\
\text { Lactona Jr.) }\end{array}$ & $\begin{array}{l}\text { SOHR } \\
\text { (scale 1-4) }\end{array}$ & $\begin{array}{l}\text { Group 1:- } \\
0.71(0.59)\end{array}$ & $\begin{array}{l}\text { Group 1:- } \\
0.79(0.65)\end{array}$ & 14 & USA & $\begin{array}{l}\text { MD and SD re- } \\
\text { ported for Group } \\
\text { 1. Mean square } \\
\text { difference was re- } \\
\text { ported for Group } \\
\text { 2. Type of brush- } \\
\text { mean Sq (F) } 1.05\end{array}$ & $\begin{array}{l}\text { No strong evidence of a differ- } \\
\text { ence in oral hygiene between } \\
\text { the } 2 \text { toothbrushes at this time } \\
\text { point. "There was a significant } \\
\text { regression in oral hygiene in } \\
\text { both groups" at this time point. } \\
\text { p. } 158 \text {. }\end{array}$ \\
\hline
\end{tabular}


Table 16. Electric versus manual toothbrushes (NRS): plaque medium term (Continued)

(1.54), "not a significant change".

Author report-

ed raw data no

longer available.

\begin{tabular}{|c|c|c|c|c|c|c|c|c|c|}
\hline $\begin{array}{l}8-20 \\
\text { weeks }\end{array}$ & $\begin{array}{l}\text { Gertenrich } \\
\text { 1967d }\end{array}$ & $\begin{array}{l}\text { Oral B Automat- } \\
\text { ic Toothbrush - } \\
\text { Arcuate actions } \\
\text { vs manual brush } \\
\text { (Py-co-pay, Hard, } \\
\text { Lactona Jr.) }\end{array}$ & $\begin{array}{l}\text { SOHR } \\
\text { (scale 1-4) }\end{array}$ & $\begin{array}{l}-0.57 \\
(0.54)\end{array}$ & $\begin{array}{l}-0.88 \\
(0.65)\end{array}$ & 42 & USA & $\begin{array}{l}\text { MD presented in } \\
\text { this study. }\end{array}$ & $\begin{array}{l}\text { A slight regression in the im- } \\
\text { provements made in oral hy- } \\
\text { giene with both the electric } \\
\text { and manual toothbrushes ( } \mathrm{P}= \\
0.001) \text {, no strong evidence of a } \\
\text { difference in oral hygiene be- } \\
\text { tween the } 2 \text { brushes. }\end{array}$ \\
\hline $\begin{array}{l}10-28 \\
\text { weeks }\end{array}$ & $\begin{array}{l}\text { Gertenrich } \\
\text { 1967b }\end{array}$ & $\begin{array}{l}\text { Oral B Automat- } \\
\text { ic Toothbrush - } \\
\text { Arcuate actions } \\
\text { vs manual brush } \\
\text { (Py-co-pay, Hard, } \\
\text { Lactona Jr.) }\end{array}$ & $\begin{array}{l}\text { SOHR } \\
\text { (scale 1-4) }\end{array}$ & $\begin{array}{l}\text { Group 1: } \\
0.24(0.55)\end{array}$ & $\begin{array}{l}\text { Group 1: } \\
0.03(1.37)\end{array}$ & 52 & USA & $\begin{array}{l}\text { MD presented } \\
\text { in this study for } \\
\text { Group } 1 \text { only. } \\
\text { Group } 2 \text { control } \\
\text { joined Group } 2 \text { in- } \\
\text { tervention after } \\
\text { week } 8 .\end{array}$ & $\begin{array}{l}\text { No strong evidence of a differ- } \\
\text { ence in oral hygiene between } \\
\text { the } 2 \text { brushes at this time point. }\end{array}$ \\
\hline $\begin{array}{l}20-34 \\
\text { weeks }\end{array}$ & $\begin{array}{l}\text { Gertenrich } \\
1967 c\end{array}$ & $\begin{array}{l}\text { Oral B Automat- } \\
\text { ic Toothbrush - } \\
\text { Arcuate actions } \\
\text { vs manual brush } \\
\text { (Py-co-pay, Hard, } \\
\text { Lactona Jr.) }\end{array}$ & $\begin{array}{l}\text { SOHR } \\
\text { (scale 1-4) }\end{array}$ & $\begin{array}{l}\text { Pooled } \\
\mathbf{0 . 3 6} \\
(\mathbf{0 . 4 9 )} \\
\text { Group 1:- } \\
0.10(0.62) ; \\
\text { Group 2: } \\
0.94(0.30)\end{array}$ & $\begin{array}{l}\text { Pooled } \\
\mathbf{0 . 6 9} \\
(\mathbf{0 . 4 7}) \\
\text { Group 1: } \\
0.32(0.61) \\
\text { Group 2: } \\
1.28(0.25)\end{array}$ & 36 & USA & $\begin{array}{l}\text { Pooled MD and } \\
\text { SD calculated } \\
\text { from the reported } \\
\text { data. }\end{array}$ & $\begin{array}{l}\text { Strong evidence of an improve- } \\
\text { ment in oral hygiene in Group } \\
2 \text { in both the electric and man- } \\
\text { ual toothbrushes }(P=0.05) \text {. No } \\
\text { strong evidence of an improve- } \\
\text { ment in either brush in Group } 1 .\end{array}$ \\
\hline
\end{tabular}

MD: mean difference; NRS: non-randomised study; S\&LPI: Silness \& Löe Plaque Index, scale 0-3; SD: standard deviation; SOHR: Subjective Oral Hygiene Rating (good, fair, poor and very poor), scale $1-4$

Gertenrich 1967b: means and SDs presented separately for two distinct groups with ID (Group 1: Down's syndrome; Group 2: intelligence quotient (IQ); $\leq 20)$, the pooled mean and SD were calculated from these data. All participants' teeth were brushed by attendants. "Although the results obtained using the automatic units in a controlled regime were more stable and generally some what better (Figure 1) compared to the results with the hand brushing on a controlled regime, it is clear that these added benefits can vanish soon after the controlled regime is discontinued," p. 148. Gertenrich 1967c: means and SDs presented separately for two distinct ID groups (Group 1: hydrocephaly and seizure unit; Group 2: paediatric unit) the pooled mean and SD were calculated from these data. All participants' teeth were brushed by attendants. Gertenrich 1967a: two distinct groups with ID (Group 1: participants with cerebral palsy with IQ 30-95; Group 2 "trainable patients" with IQ 30-50). In Group 1, all were brushed by attendants. In Group 2, half the participants were brushed by attendants the other half self-brushed. In Group 2, "The most significant improvement occurred in the group that was brushed by attendants using the automated toothbrush," p. 157. Gertenrich 1967d: all participants were self-brushers with supervision and assistance from the attendants. Bratel 1991: mean and SD presented for aided and unaided brushing, pooled mean and SDs were calculated from these data. 


\begin{tabular}{|c|c|c|c|c|c|c|c|c|c|}
\hline Duration & Study & Comparison & Index & $\begin{array}{l}\text { Intervention } \\
\text { mean/SD }\end{array}$ & $\begin{array}{l}\text { Control mean/ } \\
\text { SD }\end{array}$ & Total & Country & Comments & Summary \\
\hline 16 months & $\begin{array}{l}\text { Bratel } \\
1991\end{array}$ & $\begin{array}{l}\text { Braun 3D Elec- } \\
\text { tric toothbrush } \\
\text { vs Butler } 411 \\
\text { manual tooth- } \\
\text { brush }\end{array}$ & $\begin{array}{l}\text { S\&LPI } \\
\text { (scale 0-3) }\end{array}$ & $\begin{array}{l}\text { Pooled } 1.30 \\
(\mathbf{0 . 3 5}) \\
\text { Unaided } 1.2 \\
(0.3) ; \text { aided } 1.4 \\
(0.4)\end{array}$ & $\begin{array}{l}\text { Pooled } 1.40 \\
\text { (0.30) } \\
\text { Unaided } 1.4 \\
\text { (0.3); aided } 1.4 \\
(0.3)\end{array}$ & 23 & Sweden & $\begin{array}{l}\text { Pooled mean } \\
\text { and SD calcu- } \\
\text { lated from the } \\
\text { reported da- } \\
\text { ta. }\end{array}$ & $\begin{array}{l}\text { "No significant changes } \\
\text { concerning plaque index } \\
\text { were found in or between } \\
\text { groups after } 16 \text { months," } \\
\text { p. } 6 \text {. }\end{array}$ \\
\hline
\end{tabular}

NRS: non-randomised study; S\&LPI: Silness \& Löe Plaque Index, scale 0-3; SD: standard deviation.

Bratel 1991: mean and SD presented for aided and unaided brushing; pooled mean and SDs were calculated from these data.

\section{Table 18. Electric versus manual toothbrushes (RCTs): calculus medium term}

\begin{tabular}{|c|c|c|c|c|c|c|c|c|c|}
\hline Duration & Study & Comparison & Index & $\begin{array}{l}\text { Interven- } \\
\text { tion Mean } \\
\text { (SD) }\end{array}$ & $\begin{array}{l}\text { Control } \\
\text { Mean (SD) }\end{array}$ & Total No. & Country & Comments & Summary \\
\hline 4 months & $\begin{array}{l}\text { Kelner } \\
1963\end{array}$ & $\begin{array}{l}\text { Automated } \\
\text { electric TB } \\
\text { (Broxodent) vs } \\
\text { conventional } \\
\text { TB }\end{array}$ & $\mathrm{SCR}$ & $\begin{array}{l}\% \text { Improve- } \\
\text { ment } 13 \%\end{array}$ & $\begin{array}{l}\% \text { Improve- } \\
\text { ment 10\% }\end{array}$ & 100 & USA & $\begin{array}{l}\% \text { Improvement } \\
\text { calculated from } \\
\text { the reported da- } \\
\text { ta. }\end{array}$ & $\begin{array}{l}\text { "The automatic electric } \\
\text { toothbrush appeared signifi- } \\
\text { cantly superior to the conven- } \\
\text { tional toothbrush in improv- } \\
\text { ing deposits," p. } 108 \text {. }\end{array}$ \\
\hline 6 months & $\begin{array}{l}\text { Gar- } \\
\text { cia-Carril- } \\
\text { lo } 2016\end{array}$ & $\begin{array}{l}\text { Sonicare Easy- } \\
\text { Clean, Philips } \\
\text { electric TB vs } \\
\text { Vitis Access, } \\
\text { Dentaid manual } \\
\text { TB }\end{array}$ & $\begin{array}{l}\mathrm{BDCl} \\
\text { (scale 0-1) }\end{array}$ & $0.18(0.12)$ & $0.16(0.12)$ & 64 & Spain & $\begin{array}{l}\text { The description } \\
\text { of ID in this study } \\
\text { appeared to in- } \\
\text { clude some peo- } \\
\text { ple with an IQ > } \\
70 .\end{array}$ & $\begin{array}{l}\text { No strong evidence of a dif- } \\
\text { ference in the effect of tooth- } \\
\text { brush group on the Calculus } \\
\text { ilndex }(P=0.40) \text {. }\end{array}$ \\
\hline 6 months & Carr 1997 & $\begin{array}{l}\text { Interplak elec- } \\
\text { tric TB vs Oral B } \\
40 \text { manual TB. }\end{array}$ & $\begin{array}{l}\text { SG\&VCI } \\
\text { (scale 0-3) }\end{array}$ & $\begin{array}{l}\text { Pooled } \mathbf{0 . 7 8} \\
(\mathbf{0 . 3 9 )} \\
\text { Self } \mathrm{Br} 0.70 \\
(0.41) ; \mathrm{As}- \\
\text { sist Br } 0.91 \\
(0.37)\end{array}$ & $\begin{array}{l}\text { Pooled } 0.90 \\
\text { (0.47) } \\
\text { Self } \mathrm{Br} 0.77 \\
(0.55) ; \mathrm{As}- \\
\text { sist } \mathrm{Br} 1.23 \\
(0.37)\end{array}$ & 56 & USA & $\begin{array}{l}\text { Pooled mean and } \\
\text { SD calculated } \\
\text { from the reported } \\
\text { data. Cluster RCT }\end{array}$ & $\begin{array}{l}\text { No evidence of a difference } \\
\text { between groups for the Calcu- } \\
\text { lus Index. }\end{array}$ \\
\hline 12 months & & $\begin{array}{l}\text { Interplak elec- } \\
\text { tric TB vs Oral B } \\
40 \text { manual TB. }\end{array}$ & $\begin{array}{l}\text { SG\&VCI } \\
\text { (scale 0-3) }\end{array}$ & $\begin{array}{l}\text { Pooled } \mathbf{0 . 7 6} \\
(\mathbf{0 . 5 0 )} \\
\text { Self Br } 1.81 \\
(0.31) ; \text { As- }\end{array}$ & $\begin{array}{l}\text { Pooled } \mathbf{0 . 9 3} \\
(\mathbf{0 . 4 0 )} \\
\text { Self } \mathrm{Br} 1.81 \\
(0.31) ; \text { As- }\end{array}$ & 56 & USA & $\begin{array}{l}\text { Pooled mean and } \\
\text { SD calculated } \\
\text { from the reported } \\
\text { data. Cluster RCT. }\end{array}$ & $\begin{array}{l}\text { No evidence of a difference } \\
\text { between groups could be } \\
\text { found for the Calculus Index. }\end{array}$ \\
\hline
\end{tabular}


Table 18. Electric versus manual toothbrushes (RCTs): calculus medium term (Continued)

sist $\mathrm{Br} 1.78 \quad$ sist $\mathrm{Br} 1.78$

(0.33)

$(0.33)$

Assist Br: assisted brusher; BDCI: Bentley \& Disney Calculus Index, present or absent; ID: intellectual disability; IQ: intelligence quotient; RCT: randomised controlled trial; SD: standard deviation; SCR: Subjective Calculus Rating - none, moderate, extreme; Self Br: self-brusher; SG\&VCI: Simplified Green \& Vermillion Calculus Index, scale 0-3.

Kelner 1963: subjective data presented; the term 'deposits' was described as "the presence and amount of stain, soft and hard deposits," p. 102. \% improvement calculated from the differences between the combined 'extreme' ratings pre and post. Data also presented by age groups (aged 4-14 years, 15-32 years). Carr 1997: means and SDs presented separately for Self $\mathrm{Br}$ and Assist $\mathrm{Br}$; the pooled means and SDs were calculated from these data for the different time points.

\section{Table 19. Electric versus manual toothbrush: self-efficacy long term}

\begin{tabular}{|c|c|c|c|c|c|c|c|c|c|}
\hline Duration & Study & Comparison & Index & $\begin{array}{l}\text { Intervention } \\
\text { (yes/no) }\end{array}$ & $\begin{array}{l}\text { Control (yes/ } \\
\text { no) }\end{array}$ & $\begin{array}{l}\text { Number } \\
\text { of partici- } \\
\text { pants }\end{array}$ & Country & Comments & Summary \\
\hline 16 months & $\begin{array}{l}\text { Bratel } \\
1991\end{array}$ & $\begin{array}{l}\text { Braun 3D Electric } \\
\text { toothbrush vs But- } \\
\text { ler } 411 \text { manual } \\
\text { toothbrush }\end{array}$ & $\begin{array}{l}\text { SE (yes/ } \\
\text { no) }\end{array}$ & $\begin{array}{l}\text { Pooled } 4 / 8 \\
\text { Aided } 1 / 5 ; \text { un- } \\
\text { aided } 3 / 3\end{array}$ & $\begin{array}{l}\text { Pooled } 3 / 7 \\
\text { Aided } 2 / 3 ; \text { un- } \\
\text { aided } 1 / 4\end{array}$ & 23 & Sweden & $\begin{array}{l}\text { Data calculated for } \\
\text { pooled aided and un- } \\
\text { aided participants for } \\
\text { each group. }\end{array}$ & $\begin{array}{l}\text { No evidence } \\
\text { of a difference } \\
\text { between the } \\
\text { groups. }\end{array}$ \\
\hline
\end{tabular}

SE: self-efficacy: "Do you find it difficult to brush your patient's teeth?"

Table 20. Training of carers versus no training of carers (RCTs): gingival inflammation medium term

\begin{tabular}{|c|c|c|c|c|c|c|c|c|c|}
\hline Duration & Study & Comparison & Index & $\begin{array}{l}\text { Interven- } \\
\text { tion mean } \\
\text { (SD) }\end{array}$ & $\begin{array}{l}\text { Control } \\
\text { mean (SD) }\end{array}$ & Total & Country & $\begin{array}{l}\text { Com- } \\
\text { ments }\end{array}$ & Summary \\
\hline 8 weeks & $\begin{array}{l}\text { Phlypo } \\
2016\end{array}$ & $\begin{array}{l}\text { Carers pro- } \\
\text { vided with } \\
\text { an Informa- } \\
\text { tion booklet } \\
\text { and informa- } \\
\text { tion session } \\
\text { with practical } \\
\text { skills. }\end{array}$ & $\begin{array}{l}\text { MGI (scale } \\
0-3)\end{array}$ & $1.2(0.36)$ & $1.5(0.24)$ & 36 & Belgium & $\begin{array}{l}\text { MD data } \\
\text { were re- } \\
\text { ported; in- } \\
\text { tervention } \\
0.1 \text { (SD } \\
0.61) \text {; con- } \\
\text { trol } 0.003 \\
\text { (SD } 0.32 \text { ) }\end{array}$ & $\begin{array}{l}\text { No strong evidence of a difference was } \\
\text { found in Gingival Index between the inter- } \\
\text { vention and control group pre- and post- } \\
\text { test. }(P=0.14) \text {, However, when the indi- } \\
\text { vidual post-test data were considered, } \\
\text { there was strong evidence of a difference } \\
(P=0.02) \text { in Gingival Index between the in- } \\
\text { tervention and control group, p. } 15 \text {. }\end{array}$ \\
\hline $\begin{array}{l}6-9 \\
\text { months }\end{array}$ & $\begin{array}{l}\text { Mac Giolla } \\
\text { Phadraig } \\
2015\end{array}$ & $\begin{array}{l}\text { Staff received } \\
\text { training via } \\
\text { a pyramidal } \\
\text { training pro- }\end{array}$ & $\begin{array}{l}\text { LMGI } \\
\text { (scale 0-4) }\end{array}$ & $2.47(1.21)$ & $2.20(1.18)$ & 63 & Ireland & - & $\begin{array}{l}\text { "The difference in mean MGI and PI be- } \\
\text { tween groups was not statistically signifi- } \\
\text { cant ( } p>0.05 \text {, ANCOVA)," p. } 95 \text {. "In this in- } \\
\text { stance distal outcomes such as residents } \\
\text { oral health and hygiene did not improve in }\end{array}$ \\
\hline
\end{tabular}


Table 20. Training of carers versus no training of carers (RCTs): gingival inflammation medium term (Continued) gramme vs no

training.

the presence of proximal improvements in oral health-related knowledge, attitudes and self-efficacy, and reported behaviours of carers," p. 96.

ANCOVA: analysis of covariance; LMGI: Lobene Modified Gingival Index (non-invasive); MD: mean difference; MGI: Modified Silness \& Löe on buccal and lingual surfaces of 6 standard teeth; PI: Plaque Index; RCT: randomised controlled trial; SD: standard deviation.

Table 21. Training of carers versus no training of carers (RCTs): plaque medium term

\begin{tabular}{|c|c|c|c|c|c|c|c|c|c|}
\hline Duration & Study & Comparison & Index & $\begin{array}{l}\text { Interven- } \\
\text { tion mean } \\
\text { (SD) }\end{array}$ & $\begin{array}{l}\text { Control } \\
\text { mean (SD) }\end{array}$ & Total & Country & Comments & Summary \\
\hline 8 weeks & $\begin{array}{l}\text { Phlypo } \\
2016\end{array}$ & $\begin{array}{l}\text { Carers provided with an } \\
\text { Information booklet and } \\
\text { information session with } \\
\text { practical skills }\end{array}$ & $\begin{array}{l}\text { MPI (scale } \\
0-3)\end{array}$ & $1.4(0.38)$ & $1.5(0.24)$ & 36 & Belgium & $\begin{array}{l}\text { MD also report- } \\
\text { ed }(P=0.50)\end{array}$ & $\begin{array}{l}\text { No strong evidence of } \\
\text { a difference in MPI (P = } \\
0.35), \text { p. } 15 \text {. }\end{array}$ \\
\hline $\begin{array}{l}6-9 \\
\text { months }\end{array}$ & $\begin{array}{l}\text { Mac Giolla } \\
\text { Phadraig } \\
2015\end{array}$ & $\begin{array}{l}\text { Staff received training via } \\
\text { a pyramidal training pro- } \\
\text { gramme on oral health } \\
\text { and oral hygiene vs no } \\
\text { training }\end{array}$ & $\begin{array}{l}\text { MPI (scale } \\
0-3 \text { ) }\end{array}$ & $1.89(1.05)$ & $1.78(0.97)$ & 63 & Ireland & $\begin{array}{l}\text { Brushing was } \\
\text { carried out } \\
\text { equally by the } \\
\text { people with ID } \\
\text { and the staff. }\end{array}$ & $\begin{array}{l}\text { "The difference in mean } \\
\text { MGI and PI between } \\
\text { groups was not statis- } \\
\text { tically significant ( } p> \\
0.05, \text { ANCOVA)," p. } 95 \text {. }\end{array}$ \\
\hline
\end{tabular}

ANCOVA: analysis of covariance; ID: intellectual disability; MD: mean difference; MPI: Modified Silness \& Löe Plaque Index (6 standard teeth, no probe used, surfaces visible to the researcher); RCT: randomised controlled trial; SD: standard deviation.

Table 22. Training of carers versus no training of carers (NRS): plaque short term

\begin{tabular}{|c|c|c|c|c|c|c|c|c|c|}
\hline Duration & Study & Comparison & Index & $\begin{array}{l}\text { Interven- } \\
\text { tion mean } \\
\text { (SD) }\end{array}$ & $\begin{array}{l}\text { Control } \\
\text { mean (SD) }\end{array}$ & Total & Country & Comments & Summary \\
\hline 21 days & $\begin{array}{l}\text { Lange } \\
2000\end{array}$ & $\begin{array}{l}\text { Training of carers } \\
\text { with and without ac- } \\
\text { countability (Group } \\
1+\text { Group 2) vs no } \\
\text { training (Group 3) }\end{array}$ & $\begin{array}{l}\text { MRPDI } \\
\text { (scale 0-3) }\end{array}$ & $\begin{array}{l}\text { Pooled data } \\
\mathbf{1 . 0 9}(\mathbf{0 . 2 6 )} \\
\text { Group 1: } \\
0.23(0.02) \\
\text { Group 2: } \\
2.12(0.37)\end{array}$ & $1.78(0.45)$ & 34 & USA & $\begin{array}{l}\text { Pooled mean } \\
\text { and SD calcu- } \\
\text { lated from re- } \\
\text { ported data for } \\
\text { Group } 1 \text { and } \\
\text { Group } 2 \text {. }\end{array}$ & $\begin{array}{l}\text { The Plaque Index for Group } \\
1 \text { (with accountability) was } \\
\text { lower than that of Group } \\
2 \text { (without accountability) } \\
(P=0.004) \text { and the control } \\
\text { group }(P=0.0001) \text {. }\end{array}$ \\
\hline
\end{tabular}


Table 22. Training of carers versus no training of carers (NRS): plaque short term (Continued)

\begin{tabular}{|c|c|c|c|c|c|c|c|}
\hline $\begin{array}{l}\text { Training of carers } \\
\text { with accountability } \\
\text { (Group 1) vs training } \\
\text { without (Group 2) ac- } \\
\text { countability }\end{array}$ & $\begin{array}{l}\text { MRPDI } \\
\text { (scale 0-3) }\end{array}$ & $0.23(0.02)$ & $2.12(0.37)$ & 24 & USA & $\begin{array}{l}\text { SD calculated } \\
\text { from reported } \\
\text { data for Group } \\
1 \text { and Group } 2 .\end{array}$ & $\begin{array}{l}\text { "Fisher's LSD indicted sig- } \\
\text { nificantly lower plaque } \\
\text { indexes for experimen- } \\
\text { tal Group I vs Group } 2 \\
\text { (p=.00001)," p. } 208 .\end{array}$ \\
\hline
\end{tabular}

LSD: Least Significant Difference; MRPDI: Modified Ramfjord Periodontal Disease index - plaque only; SD: standard deviation.

Lange 2000: mean and standard error presented separately for "with accountability" (Group 1) and "without accountability" (Group 2) of trainers, and for control (Group 3); SDs calculated from these data, mean and SD pooled for Group 1 and Group 2 in Comparison 1 and compared in Comparison 2.

\section{Table 23. Training of carers versus no training of carers (NRS): plaque medium term}

\begin{tabular}{|c|c|c|c|c|c|c|c|c|c|}
\hline Duration & Study & Comparison & Index & $\begin{array}{l}\text { Interven- } \\
\text { tion mean }\end{array}$ & $\begin{array}{l}\text { Control } \\
\text { mean }\end{array}$ & Total & Country & Comments & Summary \\
\hline $\begin{array}{l}4-5 \\
\text { months }\end{array}$ & $\begin{array}{l}\text { Glassman } \\
2006\end{array}$ & $\begin{array}{l}\text { Effect of knowledge } \\
\text { acquisition on carers } \\
\text { behaviour and their } \\
\text { clients oral hygiene be- } \\
\text { haviour }\end{array}$ & $\begin{array}{l}\text { SOHI } \\
\text { (scale 0-3) }\end{array}$ & $\begin{array}{l}\text { Range 1.6- } \\
0.3\end{array}$ & $\mathrm{~N} / \mathrm{A}$ & 11 & USA & $\begin{array}{l}\text { Data in published ar- } \\
\text { ticle unclear. Multi- } \\
\text { ple attempts to con- } \\
\text { tact authors failed. }\end{array}$ & $\begin{array}{l}\text { "Improvement from } \\
\text { baseline through } \\
\text { the coaching phase } \\
\text { of from } 18.89 \% \text { to } \\
72.5 \%, " \text { p. } 43 \text {. }\end{array}$ \\
\hline
\end{tabular}

N/A: not available; NRS: non-randomised study; SOHI: Simplified Green \& Vermillion Oral Hygiene Index, debris only, scale 0-3.

Table 24. Training of carers versus no training of carers (RCTs): knowledge short and medium term

\begin{tabular}{|c|c|c|c|c|c|c|c|c|c|}
\hline Duration & Study & Comparison & Index & $\begin{array}{l}\text { Interven- } \\
\text { tion mean } \\
\text { (SD) }\end{array}$ & $\begin{array}{l}\text { Control } \\
\text { mean (SD) }\end{array}$ & Total & Country & $\begin{array}{l}\text { Com- } \\
\text { ments }\end{array}$ & Summary \\
\hline $\begin{array}{l}90 \mathrm{~min}- \\
\text { utes }\end{array}$ & $\begin{array}{l}\text { Gonzalez } \\
2013\end{array}$ & $\begin{array}{l}\text { Oral health lecture } \\
\text { with hands on train- } \\
\text { ing ( } 90 \text { minutes) vs oral } \\
\text { health discussion ( } 30 \\
\text { minutes) }\end{array}$ & $\begin{array}{l}\text { Knowledge } \\
\text { and com- } \\
\text { prehension } \\
\text { (scale } 0-1 \\
\text { for } 20 \text { ques- } \\
\text { tions) }\end{array}$ & $\begin{array}{l}\mathbf{1 8 . 2 9} \\
\mathbf{( 1 . 9 8 )} \\
\text { MD 0.061 } \\
(0.086)\end{array}$ & $\begin{array}{l}\mathbf{1 7 . 7} \\
\mathbf{( 1 . 4 9 )} \\
\text { MD 0.035 } \\
(0.094)\end{array}$ & 24 & USA & $\begin{array}{l}\text { MD report- } \\
\text { ed. As- } \\
\text { sessment } \\
\text { was im- } \\
\text { mediately } \\
\text { pre- and } \\
\text { postinter- } \\
\text { vention. }\end{array}$ & $\begin{array}{l}\text { Both groups improved their } \\
\text { knowledge from baseline; how- } \\
\text { ever, there was no significant } \\
\text { difference between the groups. }\end{array}$ \\
\hline
\end{tabular}

\begin{tabular}{|c|c|c|c|c|c|c|c|c|c|}
\hline 8 weeks & $\begin{array}{l}\text { Phlypo } \\
2016\end{array}$ & $\begin{array}{l}\text { Carers provided with } \\
\text { an information book- } \\
\text { let and information }\end{array}$ & $\begin{array}{l}\text { Knowledge } \\
\text { (scale } 0-1 \\
\text { for } 10 \text { ques- } \\
\text { tions) }\end{array}$ & $6.8(1.43)$ & $5.9(1.33)$ & 34 & Belgium & $\begin{array}{l}\text { Subgroup } \\
\text { analyses } \\
\text { reported. }\end{array}$ & $\begin{array}{l}\text { Post-test, overall no strong ev- } \\
\text { idence of differences were re- } \\
\text { ported in K-index scores be- } \\
\text { tween and within groups, p. } 6 \text {. }\end{array}$ \\
\hline
\end{tabular}


Table 24. Training of carers versus no training of carers (RCTs): knowledge short and medium term (Continued) session with practical

skills

\begin{tabular}{|c|c|c|c|c|c|c|c|c|c|}
\hline $\begin{array}{l}6.5-11 \\
\text { months }\end{array}$ & $\begin{array}{l}\text { Mac Giolla } \\
\text { Phadraig } \\
2015\end{array}$ & $\begin{array}{l}\text { Staff received training } \\
\text { via a pyramidal train- } \\
\text { ing programme vs no } \\
\text { training }\end{array}$ & $\begin{array}{l}\text { Knowledge } \\
\text { (scale } 0-1 \\
\text { for } 10 \text { ques- } \\
\text { tions) }\end{array}$ & $7.86(1.27)$ & $7.21(1.32)$ & 155 & Ireland & $\begin{array}{l}\text { Cluster } \\
\text { RCT }\end{array}$ & $\begin{array}{l}\text { Strong evidence of a difference } \\
\text { in favour of the intervention } \\
\text { was reported }(P=0.002) \text {. }\end{array}$ \\
\hline
\end{tabular}

MD: mean difference; RCT: randomised controlled group; SD: standard deviation.

Gonzalez 2013: "Both groups show an increase in scores, however, the experimental group shows almost twice the increase as the control group (0.061 vs 0.035 ). The standard deviation of both of these groups is fairly large (around 0.09)," p. 296. Mean and SD calculated from individual data provided (Table 2, p. 296). Phlypo 2016: subgroup analysis of those in the intervention group who read the information booklet compared to those in the control group who did not showed strong evidence of an improvement in knowledge $(P=0.05)$. Mac Giolla Phadraig 2015: "Using independent-samples t-test, a significant difference between control and intervention is noted for $\mathrm{K}$ Index" $(\mathrm{P}=0.002)$, $\mathrm{p} .188$ and Table 6, p. 189. The level of significance was adjusted to $P=0.026$, using the approximate false discovery rate.

Indices: multiple-choice questionnaires designed specifically for the studies for Gonzalez 2013 and Mac Giolla Phadraig 2015. Phlypo 2016 used the questionnaire used in Mac Giolla Phadraig 2015.

\section{Table 25. Training of carers versus no training of carers (RCTs): behaviour, attitude and self-efficacy medium term}

\begin{tabular}{|c|c|c|c|c|c|c|c|c|c|}
\hline Duration & Study & Comparison & Index & $\begin{array}{l}\text { Interven- } \\
\text { tion mean } \\
\text { (SD) }\end{array}$ & $\begin{array}{l}\text { Control } \\
\text { mean (SD) }\end{array}$ & Total & Country & Comments & Summary \\
\hline 8 weeks & $\begin{array}{l}\text { Phlypo } \\
2016\end{array}$ & $\begin{array}{l}\text { Carers provided } \\
\text { with an Informa- } \\
\text { tion booklet and } \\
\text { information ses- } \\
\text { sion with practical } \\
\text { skills }\end{array}$ & $\begin{array}{l}\text { BAS (14 } \\
\text { questions) }\end{array}$ & $4.7(1.72)$ & $5.2(1.49)$ & 34 & Belgium & $\begin{array}{l}\text { Subgroup analyses } \\
\text { was reported on } \\
\text { those who read the } \\
\text { information book- } \\
\text { let. }\end{array}$ & $\begin{array}{l}\text { Post-test, overall no strong } \\
\text { evidence of differences } \\
\text { were found in BAS scores } \\
\text { between and within groups } \\
(P=0.38), \text { p. } 6 .\end{array}$ \\
\hline $\begin{array}{l}8-11 \\
\text { months }\end{array}$ & $\begin{array}{l}\text { Mac Giolla } \\
\text { Phadraig } \\
2015\end{array}$ & $\begin{array}{l}\text { Staff received train- } \\
\text { ing via a pyrami- } \\
\text { dal training pro- } \\
\text { gramme vs no } \\
\text { training }\end{array}$ & $\begin{array}{l}\text { BAS (14 } \\
\text { questions) }\end{array}$ & $5.42(1.51)$ & $4.9(1.55)$ & 155 & Ireland & $\begin{array}{l}\text { Level of signifi- } \\
\text { cance adjusted to } \\
\mathrm{P}=0.028 \text {, using the } \\
\text { approximate false } \\
\text { discovery rate. }\end{array}$ & $\begin{array}{l}\text { "There was no difference in } \\
\text { BAS scale scores }(p=0.040) \\
\text { between control and inter- } \\
\text { vention at post-test," p. } 188\end{array}$ \\
\hline
\end{tabular}

BAS: behaviour (rating of -2 or +2 ) 4 questions, attitude (rating of $-2,-1,1,2) 5$ questions, self-efficacy (rating of $-2,-1,1,2$ ) 5 questions; RCT: randomised controlled trial; SD: standard deviation. 
Table 26. Training of carers versus no training of carers (RCTs): behaviour short term

\begin{tabular}{|c|c|c|c|c|c|c|c|c|c|}
\hline Duration & Study & $\begin{array}{l}\text { Compari- } \\
\text { son }\end{array}$ & Index & $\begin{array}{l}\text { Intervention } \\
\%\end{array}$ & Control \% & Total & Country & Comments & Summary \\
\hline 4 weeks & $\begin{array}{l}\text { Olmos } \\
2016\end{array}$ & $\begin{array}{l}\text { Training of } \\
\text { carers vs no } \\
\text { training of } \\
\text { carers }\end{array}$ & $\begin{array}{l}\text { Pre and } \\
\text { post sur- } \\
\text { vey of car- } \\
\text { ers }\end{array}$ & $\begin{array}{l}1.61 .10 \% \\
\text { 2. } 70.40 \% \\
\text { 3. } 54.10 \% \\
4.78 .60 \% \\
\text { 5. } 98.90 \%\end{array}$ & $\begin{array}{l}1.25 .50 \% \\
2.17 .90 \% 3 . \\
2.10 \% \\
4.29 .50 \% \\
5.64 .20 \%\end{array}$ & 193 & Germany & $\begin{array}{l}\text { 3-headed } \\
\text { toothbrush } \\
\text { (Superbrush) } \\
\text { used in this } \\
\text { study. }\end{array}$ & $\begin{array}{l}\text { Strong evidence of a difference } \\
\text { in self-reported behaviour from } \\
\text { baseline in the intervention } \\
\text { group }(P<0.01) .\end{array}$ \\
\hline
\end{tabular}

$\mathrm{RCT}$ : randomised controlled trial.

Olmos 2016: changes in behaviour assessed: 1. use of a special toothbrush, 2. use of fluoride toothpaste, 3. application of fluoride weekly, 4. toothbrushing after breakfast, and

5. duration of toothbrushing.

Table 27. Training of carers versus no training of carers (NRS): behaviour and self-efficacy medium term

\begin{tabular}{|c|c|c|c|c|c|c|c|c|c|}
\hline Duration & Study & Comparison & Index & $\begin{array}{l}\text { Interven- } \\
\text { tion mean } \\
\text { (SD) }\end{array}$ & $\begin{array}{l}\text { Control } \\
\text { mean (SD) }\end{array}$ & Total & Country & Comments & Summary \\
\hline \multirow[t]{2}{*}{100 days } & $\begin{array}{l}\text { Kissel } \\
1983\end{array}$ & $\begin{array}{l}\text { Training of car- } \\
\text { ers to assist peo- } \\
\text { ple with ID to } \\
\text { brush their teeth } \\
\text { using verbal in- } \\
\text { struction, physi- } \\
\text { cal guidance, re- } \\
\text { wards and self- } \\
\text { management } \\
\text { procedures }\end{array}$ & $\begin{array}{l}\text { Carers' } \\
\text { use of } \\
\text { training }\end{array}$ & $\begin{array}{l}\text { MD } 44 \% \\
\text { on aver- } \\
\text { age (In- } \\
\text { crease) }\end{array}$ & $\mathrm{N} / \mathrm{A}$ & 4 & USA & $\begin{array}{l}\% \text { MDs reported } \\
\text { narratively. } 2 \text { other } \\
\text { life skills were also } \\
\text { taught to other resi- } \\
\text { dents by the staff in } \\
\text { this study. }\end{array}$ & $\begin{array}{l}\text { General increases in cor- } \\
\text { rect use of all assistance } \\
\text { techniques during training; } \\
\text { physical assistance, verbal } \\
\text { instruction and rewards. All } \\
\text { staff used all types of assis- } \\
\text { tance correctly on average } \\
>94 \% \text { during the mainte- } \\
\text { nance phase, p. } 404 \text {. }\end{array}$ \\
\hline & & $\begin{array}{l}\text { Training of car- } \\
\text { ers to assist peo- } \\
\text { ple with ID to } \\
\text { brush their teeth } \\
\text { using verbal in- } \\
\text { struction, physi- } \\
\text { cal guidance, re- } \\
\text { wards and self- }\end{array}$ & $\begin{array}{l}\text { Level of } \\
\text { assis- } \\
\text { tance with } \\
\text { tooth- } \\
\text { brushing } \\
\text { needed } \\
\text { by person } \\
\text { with ID }\end{array}$ & $\begin{array}{l}\text { MD phys- } \\
\text { ical as- } \\
\text { sistance } \\
15.5 \% \text { (re- } \\
\text { duction) } \\
\text { self-ini- } \\
\text { tiation } \\
\text { "upward } \\
\text { trend" }\end{array}$ & $\mathrm{N} / \mathrm{A}$ & 4 & USA & $\begin{array}{l}2 \text { other life skills } \\
\text { were also taught to } \\
\text { other residents by } \\
\text { the staff in this study. }\end{array}$ & $\begin{array}{l}\text { Need for physical assis- } \\
\text { tance - Baseline } 47 \%-60 \% \text {, } \\
\text { reduced to } 38 \% \text { at end of } \\
\text { training. Verbal Instruction } \\
\text { was inconsistent at end of } \\
\text { training. 'Upward trends' } \\
\text { in self-initiated behaviour } \\
\text { (varied by resident), p. } 404 \text {. }\end{array}$ \\
\hline
\end{tabular}




\begin{tabular}{|c|c|c|c|c|c|c|c|c|c|}
\hline & & $\begin{array}{l}\text { management } \\
\text { procedures }\end{array}$ & & & & & & & \\
\hline \multirow[t]{3}{*}{$\begin{array}{l}4-5 \\
\text { months }\end{array}$} & \multirow[t]{3}{*}{$\begin{array}{l}\text { Glassman } \\
2006\end{array}$} & $\begin{array}{l}\text { Effect of knowl- } \\
\text { edge acquisition } \\
\text { on carers' behav- } \\
\text { iour }\end{array}$ & $\begin{array}{l}\text { Care- } \\
\text { givers' } \\
\text { presence } \\
\text { (yes/no) } \\
\text { during the } \\
\text { brushing } \\
\text { session }\end{array}$ & $\begin{array}{l}\text { MD 37- } \\
89 \% \text { (in- } \\
\text { crease) }\end{array}$ & $\mathrm{N} / \mathrm{A}$ & 11 & USA & $\begin{array}{l}\text { Data in Figure } 1 \text { pre- } \\
\text { sented unclearly in } \\
\text { published article. } \\
\text { Multiple attempts } \\
\text { to contact authors } \\
\text { failed. Data present- } \\
\text { ed as a mean for } \\
\text { each phase of the } \\
\text { training (classroom } \\
\text { sessions and coach- } \\
\text { ing sessions) in the } \\
\text { text. }\end{array}$ & $\begin{array}{l}\text { "The phase mean for care- } \\
\text { giver presence during oral } \\
\text { hygiene sessions was } 100 \% \\
\text { for all clients after the } \\
\text { coaching phase," p. } 42 \text {. }\end{array}$ \\
\hline & & $\begin{array}{l}\text { Duration of } \\
\text { brushing }\end{array}$ & $\begin{array}{l}\text { Seconds } \\
\text { spent } \\
\text { brushing }\end{array}$ & $\begin{array}{l}\text { MD 484\% } \\
\text { (increase) }\end{array}$ & $\mathrm{N} / \mathrm{A}$ & 11 & USA & $\begin{array}{l}\text { Data presented in } \\
\text { Figure } 1 \text { unclear in } \\
\text { published article. } \\
\text { Multiple attempts } \\
\text { to contact authors } \\
\text { failed. }\end{array}$ & $\begin{array}{l}\text { Duration of brushing in- } \\
\text { creased from a phase mean } \\
\text { of } 20.5-73.8 \text { seconds at } \\
\text { baseline to } 57.9-215.4 \text { sec- } \\
\text { onds after coaching, p. } 42 \text {. }\end{array}$ \\
\hline & & $\begin{array}{l}\text { Carers perception } \\
\text { of client's apti- } \\
\text { tudes }\end{array}$ & $\begin{array}{l}\text { Social } \\
\text { Valida- } \\
\text { tion Ques- } \\
\text { tionnaire } \\
\text { (scale 0-6) }\end{array}$ & $\begin{array}{l}\text { MD 0.0- } \\
0.75\end{array}$ & $\mathrm{~N} / \mathrm{A}$ & 11 & USA & - & $\begin{array}{l}\text { "The average scores showed } \\
\text { only a slight increase," p. } \\
43 .\end{array}$ \\
\hline
\end{tabular}

ID: intellectual disability; N/A: not available; NRS: non-randomised study.

Glassman 2006: carers received training in the classroom (training phase) and were also provided with ongoing coaching and feedback (coaching phase) during their oral hygiene care sessions with the people with ID for whom they cared.

Table 28. Training of people with ID versus no training of people with ID (RCTs): gingival inflammation short term

\begin{tabular}{|c|c|c|c|c|c|c|c|c|}
\hline Duration & Study & Comparison & Index & $\begin{array}{l}\text { Interven- } \\
\text { tion mean }\end{array}$ & $\begin{array}{l}\text { Control } \\
\text { mean (SD) }\end{array}$ & Total & Country & $\begin{array}{l}\text { Com- } \\
\text { ments }\end{array}$ \\
\hline
\end{tabular}

(SD)

\begin{tabular}{|c|c|c|c|c|c|c|c|c|c|}
\hline $6 / 7$ weeks & $\begin{array}{l}\text { Lange } \\
1985\end{array}$ & $\begin{array}{l}\text { Self-modelling train- } \\
\text { ing in oral hygiene } \\
\text { skills with daily use } \\
\text { of plaque disclosing } \\
\text { agents vs no train- }\end{array}$ & $\begin{array}{l}\text { S\&LGI } \\
\text { (scale 0-3) }\end{array}$ & $\begin{array}{l}\text { Pooled } \\
\text { data } 0.98 \\
(0.54)\end{array}$ & $\begin{array}{l}\text { Pooled } \\
\text { data } 1.26 \\
(0.46)\end{array}$ & 10 & USA & $\begin{array}{l}\text { Mean and } \\
\text { SD calcu- } \\
\text { lated from } \\
\text { individual } \\
\text { means }\end{array}$ & $\begin{array}{l}\text { "The gingival indices [for those in } \\
\text { treatment] improved from } 12 \% \text { to } \\
50 \%, " \text { p. } 260 \text {. The results of the Gin- } \\
\text { gival Index for the control group } \\
\text { was variable, with slight improve- }\end{array}$ \\
\hline
\end{tabular}


Table 28. Training of people with ID versus no training of people with ID (RCTs): gingival inflammation short term (Continued)

ing but daily plaque

ment, which was attributed to daiscoring ly brushing.

ID: intellectual disability; RCT: randomised controlled trial; S\&LGI: Silness \& Löe Gingival Index; SD: standard deviation.

Lange 1985: data presented by individuals, pooled mean and SD calculated from these data.

Table 29. Training of people with ID versus no training of people with ID (RCTs): plaque short term

\begin{tabular}{|c|c|c|c|c|c|c|c|c|c|}
\hline Duration & Study & Comparison & Index & $\begin{array}{l}\text { Interven- } \\
\text { tion mean } \\
\text { (SD) }\end{array}$ & $\begin{array}{l}\text { Control } \\
\text { mean (SD) }\end{array}$ & Total & Country & Comments & Summary \\
\hline 5 weeks & $\begin{array}{l}\text { Albino } \\
1979\end{array}$ & $\begin{array}{l}\text { Desensitisation ses- } \\
\text { sion and } 11 \text { practical } \\
\text { training sessions in } \\
\text { oral hygiene vs a de- } \\
\text { sensitisation session } \\
\text { only }\end{array}$ & $\begin{array}{l}\text { K\&API } \\
\text { (scale 0-5) }\end{array}$ & 2.31 & 3.04 & 26 & USA & $\begin{array}{l}\text { Data present- } \\
\text { ed is the Ad- } \\
\text { justed Post } \\
\text { Test scores. } \\
\text { Some confu- } \\
\text { sion re data } \\
\text { between the } 2 \\
\text { reports. }\end{array}$ & $\begin{array}{l}\text { "Indicating a statistically sig- } \\
\text { nificant change for the experi- } \\
\text { mental group compared to the } \\
\text { control group," Schwartz, p. } 20 \text {. } \\
\text { Analysis of covariance: } F=6.38 \text {; } \\
d f=1 / 23 ; P<0.025 \text {. Albino, p. } \\
27 \text {. }\end{array}$ \\
\hline 6/7 weeks & $\begin{array}{l}\text { Lange } \\
1985\end{array}$ & $\begin{array}{l}\text { Self-modelling train- } \\
\text { ing in oral hygiene } \\
\text { skills with daily use } \\
\text { of plaque disclosing } \\
\text { agents vs no train- } \\
\text { ing but daily plaque } \\
\text { scoring }\end{array}$ & $\begin{array}{l}\text { G\&VPI } \\
\text { (scale 0-3) }\end{array}$ & $\begin{array}{l}\text { Pooled } \\
\text { data } 0.35 \\
(0.46)\end{array}$ & $\begin{array}{l}\text { Pooled } \\
\text { data } 0.82 \\
(0.24)\end{array}$ & 10 & USA & $\begin{array}{l}\text { Mean and SD } \\
\text { calculated } \\
\text { from individ- } \\
\text { ual means. }\end{array}$ & $\begin{array}{l}\text { The plaque level reduced for } \\
\text { the intervention group from } \\
\text { baseline to follow-up by } 30 \% \text {, } \\
\text { p. } 260 \text {. There was a general in- } \\
\text { crease in plaque levels from } \\
\text { baseline in the control group. }\end{array}$ \\
\hline
\end{tabular}

df: degrees of freedom; G\&VPI: Green \& Vermillion Plaque Index; ID: intellectual disability; K\&API: Kobayashi \& Ash Plaque Index (six teeth scored, facial and lingual surfaces only); RCT: randomised controlled trial; SD: standard deviation.

Lange 1985: data presented by individuals, pooled mean and SD calculated from these data.

Table 30. Training of people with ID versus no training of people with ID (NRS): self-efficacy medium and long term

\begin{tabular}{|c|c|c|c|c|c|c|c|c|c|}
\hline Duration & Study & Comparison & Index & $\begin{array}{l}\text { Interven- } \\
\text { tion mean } \\
\text { (SD) }\end{array}$ & $\begin{array}{l}\text { Control } \\
\text { mean (SD) }\end{array}$ & Total & Country & Comments & Summary \\
\hline Unclear & $\begin{array}{l}\text { Abramson } \\
1972\end{array}$ & $\begin{array}{l}\text { Oral hygiene training } \\
\text { for people with ID us- } \\
\text { ing discrimination, }\end{array}$ & $\begin{array}{l}\text { Steps in } \\
\text { a tooth- } \\
\text { brushing }\end{array}$ & $\begin{array}{l}\text { Pooled } \\
\text { data MD }\end{array}$ & $\mathrm{N} / \mathrm{A}$ & 8 & USA & $\begin{array}{l}\text { Group mean } \\
\text { and SD calcu- }\end{array}$ & $\begin{array}{l}\text { 1-tailed t-tests for correlated } \\
\text { data showed strong evidence } \\
\text { of positive MDs in toothbrush- }\end{array}$ \\
\hline
\end{tabular}


Table 30. Training of people with ID versus no training of people with ID (NRS): self-efficacy medium and long term (Continued)

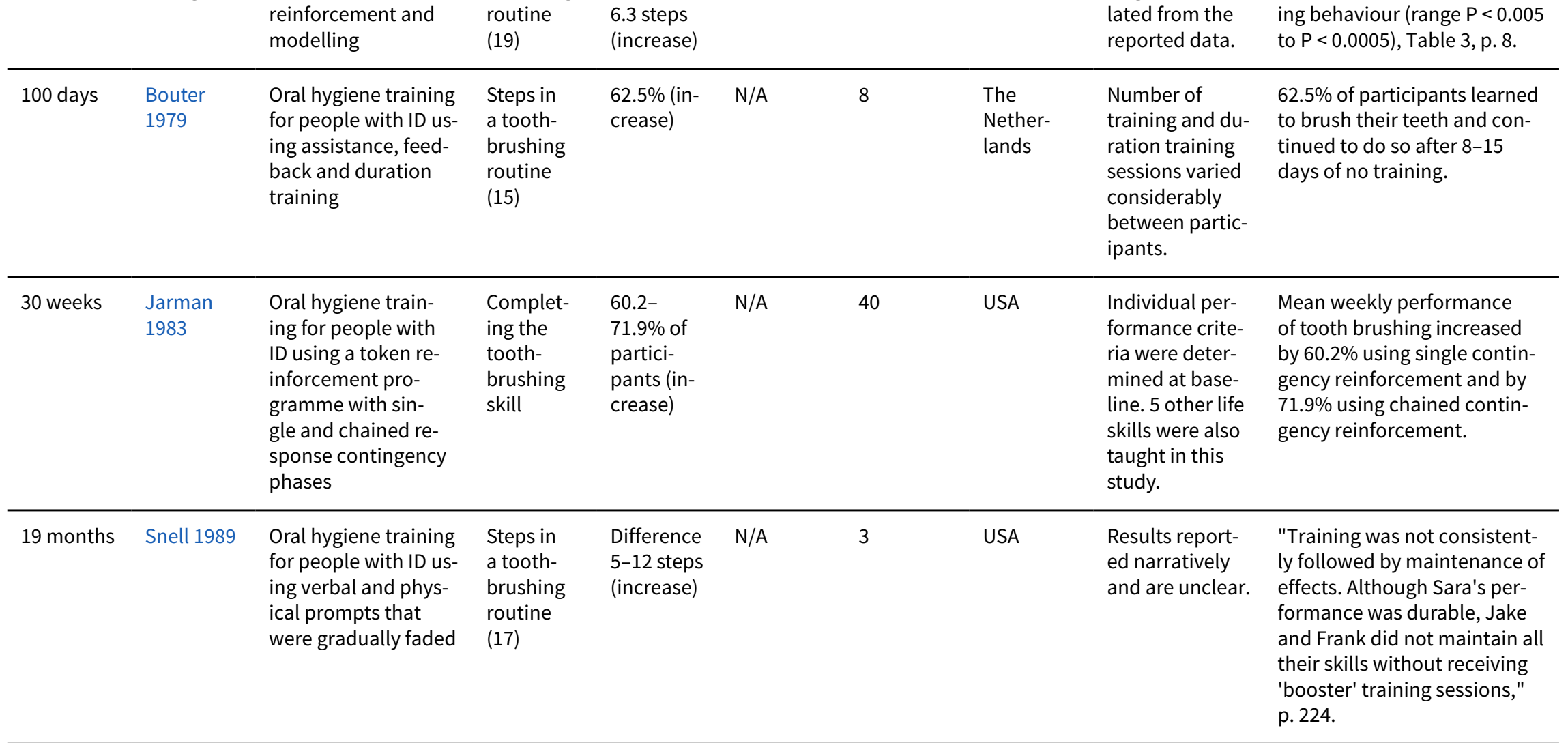

ID: intellectual disability; MD: mean difference; N/A: not available; NRS: non-randomised study; SD: standard deviation.

Abramson 1972: mean individual data reported (no SD reported), group mean and SD calculated from these data.

Table 31. One-, three- or six-monthly dental recall versus no regular dental recall (RCTs): gingival inflammation long term

\begin{tabular}{|c|c|c|c|c|c|c|c|c|c|}
\hline Duration & Study & Comparison & Index & $\begin{array}{l}\text { Intervention } \\
\text { mean (SD) }\end{array}$ & $\begin{array}{l}\text { Control } \\
\text { mean (SD) }\end{array}$ & Total & Country & Comments & Summary \\
\hline 24 months & Shaw 1991 & $\begin{array}{l}\text { 1-, 3- and 6- } \\
\text { monthly dental } \\
\text { recall vs no regu- } \\
\text { lar dental recall }\end{array}$ & $\begin{array}{l}\text { WHOGPI } \\
\text { (scale 0-2) }\end{array}$ & $\begin{array}{l}\text { R1: } 0.3(0.8) ; \mathrm{R} 3: \\
0.4(0.7) ; \mathrm{R} 6: 0.5 \\
(0.9)\end{array}$ & $0.9(1.4)$ & 304 & UK & $\begin{array}{l}\text { All participants } \\
\text { also had su- } \\
\text { pervised daily } \\
\text { toothbrushing. }\end{array}$ & $\begin{array}{l}\text { Strong evidence of differ- } \\
\text { ences between the groups } \\
\text { for Code } 1 \mathrm{GPI} \text { at } 3,6,12 \text { and } \\
24 \text { months }(\mathrm{P}<0.01-0.05) \text {. }\end{array}$ \\
\hline
\end{tabular}


GPI: Gingival Pocket Index; R1: one-month recall; R3: three-month recall; R6: six-month recall; RCT: randomised controlled trial; SD: standard deviation; WHOGPI: World Health Organization Gingival Pocket Index, scale 0-2 (0: 0-3.5 mm; : > 3.5-5.5 mm; 2: > $5.5 \mathrm{~mm})$.

Shaw 1991: data also reported for 3, 6, 12 and 18 months, not reported here as participant numbers were not provided. Data for "mean number of sextants scored as Code 1." No strong evidence of a difference for Code 2 , but the actual number of effected sextants was very low.

\section{Table 32. One-, three- or six-monthly dental recall versus no regular dental recall (RCTs): gingival bleeding long term}

\begin{tabular}{|c|c|c|c|c|c|c|c|c|c|}
\hline Duration & Study & Comparison & Index & $\begin{array}{l}\text { Intervention } \\
\text { mean (SD) }\end{array}$ & $\begin{array}{l}\text { Control } \\
\text { mean (SD) }\end{array}$ & Total & Country & Comments & Summary \\
\hline 24 months & Shaw 1991 & $\begin{array}{l}\text { 1-, 3- and 6- } \\
\text { monthly dental } \\
\text { recall vs no reg- } \\
\text { ular dental re- } \\
\text { call }\end{array}$ & $\begin{array}{l}\text { WHOBI } \\
\text { (scale 0-1) }\end{array}$ & $\begin{array}{l}\text { R1: } 2.2(1.9) ; \\
\text { R3: } 2.3(1.9) ; \\
\text { R6: } 2.6(1.8)\end{array}$ & $2.4(2.0)$ & 304 & UK & $\begin{array}{l}\text { All partici- } \\
\text { pants also } \\
\text { had super- } \\
\text { vised daily } \\
\text { toothbrush- } \\
\text { ing. }\end{array}$ & $\begin{array}{l}\text { Strong evidence of differences } \\
\text { in } \mathrm{BI} \text { at the } 3-, 6 \text { - and } 12 \text {-month } \\
\text { time points }(\mathrm{P}<0.01) \text { were not } \\
\text { observed at } 18 \text { or } 24 \text { months }\end{array}$ \\
\hline
\end{tabular}

BI: Bleeding Index; R1: one-month recall; R3: three-month recall; R6: six-month recall; RCT: randomised controlled trial; SD: standard deviation; WHOBI: World Health Organization Bleeding Index, scale $0-1$; presence or absence of bleeding after 20 seconds.

Shaw 1991: data also reported for 3, 6,12 and 18 months, not reported here as participant numbers not provided.

Table 33. One-, three- or six-monthly dental recall versus no regular dental recall (RCTs): plaque long term

\begin{tabular}{|c|c|c|c|c|c|c|c|c|c|}
\hline Duration & Study & Comparison & Index & $\begin{array}{l}\text { Intervention } \\
\text { mean (SD) }\end{array}$ & $\begin{array}{l}\text { Control } \\
\text { mean (SD) }\end{array}$ & Total & Country & Comments & Summary \\
\hline 24 months & Shaw 1991 & $\begin{array}{l}1-, 3 \text { - and 6- } \\
\text { monthly dental } \\
\text { recall vs no reg- } \\
\text { ular dental re- } \\
\text { call }\end{array}$ & $\begin{array}{l}\text { WHOPI } \\
\text { (scale 0-3) }\end{array}$ & $\begin{array}{l}\text { R1: } 1.2(1.7) ; \\
\text { R3: } 1.3(1.7) ; \\
\text { R6: } 1.3(1.5)\end{array}$ & $2.0(1.9)$ & 304 & UK & $\begin{array}{l}\text { All partici- } \\
\text { pants also } \\
\text { had super- } \\
\text { vised daily } \\
\text { toothbrush- } \\
\text { ing. }\end{array}$ & $\begin{array}{l}\text { Strong evidence of differences for } \\
\text { Plaque Index at } 12(P<0.01) \text { and } \\
24 \text { months }(P<0.05) \text {; the great- } \\
\text { est differences were seen in the } \\
1 \text { - and 3-monthly intervals. }\end{array}$ \\
\hline
\end{tabular}

R1: one-month recall; R3: three-month recall; R6: six-month recall; SD: standard deviation; WHOPI: World Health Organization Plaque Index; data were for "mean number of sextants scored as Code 2."

Shaw 1991: data also reported for 3, 6, 12 and 18 months, not reported here as participant numbers not provided. "The difference between the one-monthly and three monthly hygienist input (Recall visit) were not clinically very apparent after two years," p. 144.

Table 34. One-, three- or six-monthly dental recall versus no regular dental recall (RCTs): calculus long term

Duration Study Comparison Index Intervention control Total Country Comments Summary


Table 34. One-, three- or six-monthly dental recall versus no regular dental recall (RCTs): calculus long term (Continued)

24 months Shaw 1991

1-, 3- and 6-
monthly dental
recall vs no regu-
lar dental recall

WHOCl

$3.6(2.1)$

304

UK

All participants

also had su-

pervised daily

Strong evidence of differences in confidence in

lar dental recall

R1: one-month recall; R3: three-month recall; R6: six-month recall; RCT: randomised controlled trial; WHOCI: World Health Organization Calculus Index, scale 0-1 (presence or absence).

Shaw 1991: data also reported at 3, 6, 12 and 18 months, not reported here as participant numbers not provided.

Table 35. Individual clinical photographs used as motivators compared to no motivators for people with ID (RCTs): plaque medium term

\begin{tabular}{|c|c|c|c|c|c|c|c|c|c|}
\hline Duration & Study & Comparison & Index & $\begin{array}{l}\text { Interven- } \\
\text { tion mean } \\
\text { (SD) }\end{array}$ & $\begin{array}{l}\text { Control } \\
\text { mean (SD) }\end{array}$ & Total & Country & Comments & Summary \\
\hline \multirow[t]{2}{*}{6 months } & \multirow[t]{2}{*}{$\begin{array}{l}\text { Bickley } \\
1990\end{array}$} & \multirow{2}{*}{$\begin{array}{l}\text { Discussion of } \\
\text { clinical pho- } \\
\text { tographic } \\
\text { records vs no } \\
\text { discussion }\end{array}$} & $\begin{array}{l}\text { WHOPI } \\
\text { (scale } 0- \\
12 \text { ) }\end{array}$ & $7.3(2.8)$ & $7.4(2.0)$ & 29 & UK & $\begin{array}{l}\text { Disclosing so- } \\
\text { lution was also } \\
\text { used. }\end{array}$ & $\begin{array}{l}\text { "There was no significant changes af- } \\
\text { ter } 6 \text { months in either of the groups," } \\
\text { p. } 4 .\end{array}$ \\
\hline & & & ESI (\%) & $\begin{array}{l}37.7 \% \\
(18.2)\end{array}$ & $\begin{array}{l}40.3 \% \\
(26.4)\end{array}$ & 29 & UK & $\begin{array}{l}\text { Disclosing so- } \\
\text { lution was also } \\
\text { used. }\end{array}$ & $\begin{array}{l}\text { "The test group was considerably } \\
\text { lower but just failed to achieve sta- } \\
\text { tistical significance ( } t=1.721, \mathrm{df} \\
=15), " \text { p. } 4 \text {. }\end{array}$ \\
\hline
\end{tabular}

df: degrees of freedom; ESI: Extrinsic Stain Index Shaw \& Murray (\% plaque on anterior teeth); SD: standard deviation; WHOPI: World Health Organization Plaque Index (4 surfaces per tooth, scored $0-3$ resulting in a scale of $0-12$ ).

Table 36. Frequency of brushing (RCTs): gingival inflammation short term

\begin{tabular}{|c|c|c|c|c|c|c|c|c|c|}
\hline Duration & Study & $\begin{array}{l}\text { Compari- } \\
\text { son }\end{array}$ & Index & $\begin{array}{l}\text { Intervention } \\
\text { mean }\end{array}$ & $\begin{array}{l}\text { Control } \\
\text { mean }\end{array}$ & Total & Country & Comments & Summary \\
\hline \multirow[t]{2}{*}{21 days } & $\begin{array}{l}\text { Swallow } \\
1969\end{array}$ & $\begin{array}{l}\text { Daily vs } \\
\text { twice week- } \\
\text { ly brushing }\end{array}$ & $\begin{array}{l}\text { B\&LL\&SGI } \\
\text { (scale 0-6) }\end{array}$ & $\begin{array}{l}\text { Pooled } 1.84 \\
\text { Electric } 1.24 \\
\text { manual } 2.43\end{array}$ & $\begin{array}{l}\text { Pooled } 2.05 \\
\text { Electric } \\
1.59 ; \text { manu- } \\
\text { al } 2.51\end{array}$ & 40 & UK & $\begin{array}{l}\text { Pooled mean } \\
\text { calculated from } \\
\text { reported data }\end{array}$ & $\begin{array}{l}\text { Brushing twice a week reduced } \\
\text { gingival disease by approxi- } \\
\text { mately } 46 \% \text { and brushing daily } \\
\text { by } 51 \%, \text { p. } 378 \text {. }\end{array}$ \\
\hline & & $\begin{array}{l}\text { Daily vs } \\
\text { once weekly } \\
\text { brushing }\end{array}$ & $\begin{array}{l}\text { B\&LL\&SGI } \\
\text { (scale 0-6) }\end{array}$ & $\begin{array}{l}\text { Pooled } 1.84 \\
\text { Electric } 1.24 \\
\text { manual } 2.43\end{array}$ & $\begin{array}{l}\text { Pooled } \\
3.42\end{array}$ & 40 & UK & $\begin{array}{l}\text { Pooled mean } \\
\text { calculated from } \\
\text { reported data }\end{array}$ & $\begin{array}{l}\text { Brushing once a week reduced } \\
\text { gingival disease by approxi- } \\
\text { mately } 26 \% \text { and brushing daily } \\
\text { by } 51 \%, \text { p. } 378 \text {. }\end{array}$ \\
\hline
\end{tabular}


Table 36. Frequency of brushing (RCTs): gingival inflammation short term (Continued)

Electric

3.26; manu-

al 3.57

\begin{tabular}{|c|c|c|c|c|c|c|c|}
\hline $\begin{array}{l}\text { Daily brush- } \\
\text { ing vs usual } \\
\text { care }\end{array}$ & $\begin{array}{l}\text { B\&LL\&SGI } \\
\text { (scale 0-6) }\end{array}$ & $\begin{array}{l}\text { Pooled } 1.84 \\
\text { Electric } 1.24 ; \\
\text { manual } 2.43\end{array}$ & 4.27 & 60 & UK & $\begin{array}{l}\text { Pooled mean } \\
\text { calculated from } \\
\text { reported data }\end{array}$ & $\begin{array}{l}\text { Brushing daily differed strong- } \\
\text { ly from usual care - decreasing } \\
\text { levels of gingivitis by 51\%, p. } \\
378 \text {. }\end{array}$ \\
\hline
\end{tabular}

B\&LL\&SGI: buccal and labial surfaces Löe \& Silness, scale 0-6; RCT: randomised controlled trial.

Swallow 1969: data presented separately for electric and manual toothbrushes, labial and buccal surfaces, and males and females; no SD reported. The data for the females using the electric toothbrush daily and twice weekly appeared unrealistically low; author could not be contacted to clarify. Pooled means were calculated from these data. Usual care group "rarely received any regular form of oral hygiene."

Table 37. Disclosing agent versus no disclosing agent (NRS): gingival inflammation short term

\begin{tabular}{|c|c|c|c|c|c|c|c|c|c|}
\hline Duration & Study & Comparison & Index & $\begin{array}{l}\text { Interven- } \\
\text { tion mean }\end{array}$ & $\begin{array}{l}\text { Control } \\
\text { mean }\end{array}$ & Total & Country & Comments & Summary \\
\hline 10 days & $\begin{array}{l}\text { Teitel- } \\
\text { baum } \\
2009\end{array}$ & $\begin{array}{l}\text { Toothpaste with } \\
\text { a disclosing } \\
\text { agent vs a regular } \\
\text { toothpaste }\end{array}$ & $\begin{array}{l}\text { GBI (scale } \\
0-1 \text { ) }\end{array}$ & $\begin{array}{l}\% \text { mean } \\
\text { difference } \\
18\end{array}$ & $\begin{array}{l}\% \text { mean } \\
\text { difference } \\
8\end{array}$ & 40 & Brazil & $\begin{array}{l}\text { Cross-over trial. } \\
\text { Data presented } \\
\text { for } 2 \text { arms com- } \\
\text { bined. }\end{array}$ & $\begin{array}{l}\text { Strong evidence of a difference in } \\
\text { Bleeding Index in favour of the in- } \\
\text { tervention }(P<0.001), \text { p. } 466 \text {. }\end{array}$ \\
\hline
\end{tabular}

GBI: Gingival Bleeding Index Ainamo \& Bay (present or absent); NRS: non-randomised study.

Teitelbaum 2009: included two other experimental groups using chlorhexidine, which are not relevant to this review. The toothpaste containing a plaque-disclosing agent produced a reduction in gingival inflammation similar to the dentifrice with chlorhexidine (18\%) (not reported here). Exact data in Figure 2 unclear; requested from authors, but no response.

Table 38. Disclosing agent versus no disclosing agent (NRS): plaque short term

\begin{tabular}{|c|c|c|c|c|c|c|c|c|c|}
\hline Duration & Study & Comparison & Index & $\begin{array}{l}\text { Interven- } \\
\text { tion mean }\end{array}$ & $\begin{array}{l}\text { Control } \\
\text { mean }\end{array}$ & Total & Country & Comments & Summary \\
\hline 10 days & $\begin{array}{l}\text { Teitel- } \\
\text { baum } \\
2009\end{array}$ & $\begin{array}{l}\text { Toothpaste with } \\
\text { a disclosing agent } \\
\text { vs a regular tooth- } \\
\text { paste }\end{array}$ & $\begin{array}{l}\text { SG\&VOHI } \\
\text { (scale 0-3) }\end{array}$ & $\begin{array}{l}\% \text { mean dif- } \\
\text { ference } 65\end{array}$ & $\begin{array}{l}\% \text { mean } \\
\text { difference } \\
15\end{array}$ & 40 & Brazil & $\begin{array}{l}\text { Cross-over trial. } \\
\text { Data presented } \\
\text { for } 2 \text { arms com- } \\
\text { bined. }\end{array}$ & $\begin{array}{l}\text { Strong evidence of a dif- } \\
\text { ference in plaque levels in } \\
\text { favour of the intervention ( } P \\
<0.0001) \text {, p. } 466 \text {. }\end{array}$ \\
\hline
\end{tabular}

NRS: non-randomised study; SG\&VOHI: Simplified Green \& Vermillion Oral Hygiene Index - plaque only. Scoring of disclosed plaque on buccal surfaces of 4 teeth and lingual 
Teitelbaum 2009: included two other experimental groups not relevant to this review. Exact data in Figure 2 unclear. Requested from authors, but no response.

Table 39. Individualised care plan versus usual care (NRS): plaque medium term

\begin{tabular}{|c|c|c|c|c|c|c|c|c|c|}
\hline Duration & Study & $\begin{array}{l}\text { Compari- } \\
\text { son }\end{array}$ & Index & $\begin{array}{l}\text { Interven- } \\
\text { tion mean }\end{array}$ & $\begin{array}{l}\text { Control } \\
\text { mean }\end{array}$ & Total & Country & Comments & Summary \\
\hline \multirow[t]{2}{*}{12 months } & \multirow[t]{2}{*}{$\begin{array}{l}\text { Altabet } \\
2003\end{array}$} & \multirow{2}{*}{$\begin{array}{l}\text { Individu- } \\
\text { alised oral } \\
\text { care plan } \\
\text { vs usual } \\
\text { care (gen- } \\
\text { eral oral } \\
\text { care poli- } \\
\text { cy) }\end{array}$} & $\begin{array}{l}\text { SOHR } \\
\text { (scale 1-5) }\end{array}$ & 2.97 & 3.10 & 79 & USA & $\begin{array}{l}\text { The intervention group was } \\
\text { monitored, monthly - quar- } \\
\text { terly, with staff feedback } \\
\text { and review as required. No } \\
\text { monitoring of the control } \\
\text { group. }\end{array}$ & $\begin{array}{l}\text { "People in the treatment } \\
\text { group showed statistically } \\
\text { significant improvement, } \mathrm{t} \\
(39)=3.82, \mathrm{p}<.001), " \text { p. } 442 \text {. }\end{array}$ \\
\hline & & & $\begin{array}{l}\text { Improve- } \\
\text { ment dif- } \\
\text { ferential }\end{array}$ & $38 \%$ & $5 \%$ & 79 & USA & $\begin{array}{l}\text { The intervention group was } \\
\text { monitored, monthly - quar- } \\
\text { terly, with staff feedback } \\
\text { and review as required. No } \\
\text { monitoring of the control } \\
\text { group. }\end{array}$ & $\begin{array}{l}\text { "The results indicate that } \\
\text { practices can be imple- } \\
\text { mented to improve oral hy- } \\
\text { giene in individuals with } \\
\text { mental retardation living at } \\
\text { a state residential facility," } \\
\text { p. } 442 \text {. }\end{array}$ \\
\hline
\end{tabular}

NRS: non-randomised study; SOHR: Subjective Oral Hygiene Rating, scale 1-5 (1 excellent: no plaque on teeth, 2 good; plaque present on less than $15 \%$ of total tooth surface (tts), 3 fair; plaque present on $15-50 \%$ of tts, 4 poor; plaque present on $50-90 \%$ of $\mathrm{tts}, 5$ plaque present on greater than $90 \%$ of $\mathrm{tts}$ ), p. 441 . Numerical ratings were averaged over the two designated time periods. Improvement differential: difference between percentage of individuals with improvement rating minus the $\%$ individuals with poorer rating over same time period. 


\section{AP PE N D I C E S}

\section{Appendix 1. Search strategies for identification of studies}

\section{Cochrane Oral Health's Trials Register search strategy}

Cochrane Oral Health's Trials Register is available via the Cochrane Register of Studies. For information on how the register is compiled, see oralhealth.cochrane.org/trials

1. MESH DESCRIPTOR Intellectual disability EXPLODE ALL AND INREGISTER

2. MESH DESCRIPTOR Developmental disability AND INREGISTER

3. ((intellectual or mental) and "developmental disorder") AND INREGISTER

4. ((intellectual* or mental* or learning) NEAR5 (disabl* or disabilit* or deficien* or impair* or handicap*)) AND INREGISTER

5. ((deficien* or low*) NEAR3 (cognition or "cognitive function*" or reason* or intelligence))

6. ("special needs" or (special NEAR3 child\$) or retard* or "slow learner*") AND INREGISTER

7. ("Down* syndrome" or mongol* or "De Lange syndrome" or "Prader Willi syndrome" or "Labhart Willi syndrome" or "Royer syndrome" or "Rubinstein-Taybe syndrome" or "Rubinstein syndrome" or "WAGR syndrome" or "Williams syndrome" or "Broad Thumb Hallux syndrome") AND INREGISTER

8. \#1 or \#2 or \#3 or \#4 or \#5 or \#6 or \#7

\section{Cochrane Central Register of Controlled Clinical Trials (CENTRAL) search strategy}

1. MESH DESCRIPTOR Dental care for disabled AND CENTRAL:TARGET

2. MESH DESCRIPTOR Oral hygiene EXPLODE ALL AND CENTRAL:TARGET

3. MESH DESCRIPTOR Oral health AND CENTRAL:TARGET

4. MESH DESCRIPTOR Periodontal diseases EXPLODE ALL AND

5. CENTRAL:TARGET

6. MESH DESCRIPTOR Periodontics EXPLODE ALL AND CENTRAL:TARGET

7. (periodont ${ }^{\star}$ or gingiv ${ }^{\star}$ ) AND CENTRAL:TARGET

8. MESH DESCRIPTOR Dental Health Surveys EXPLODE ALL AND CENTRAL:TARGET

9. (toothbrush* or tooth-brush ${ }^{\star}$ or floss* or "chewing stick*" or "wood stick*" or toothpick ${ }^{\star}$ ) AND CENTRAL:TARGET

10.(caries or carious) AND CENTRAL:TARGET

11.MESH DESCRIPTOR Dental caries EXPLODE ALL AND CENTRAL:TARGET

12.((dental or oral or mouth or interdental or interproximal or tooth or teeth or orthodontic or denture* or brace* or bracket $\left.{ }^{\star}\right)$ NEAR3 (irrigat* or clean $^{\star}$ or brush* or clens $^{\star}$ or aid*)) AND CENTRAL:TARGET

13.((oral or dental) NEAR2 (hygiene or care)) AND CENTRAL:TARGET

14. ((mouth or teeth) NEAR3 care) AND CENTRAL:TARGET(plaque* NEAR5 (remov* or control*)) AND CENTRAL:TARGET

15.("dental plaque index" or "dental plaque indices" or "DMF* index" or "DMF indices" or "dmf* index" or "dmf* indices" or "periodontal index" or "periodontal indices" or "oral hygiene index" or "oral hygiene indices" or "gingival index") AND CENTRAL:TARGET

16.MESH DESCRIPTOR Dental plaque AND CENTRAL:TARGET

17.MESH DESCRIPTOR Health Education, Dental AND CENTRAL:TARGET

18.((health* NEAR3 promot*) and (dental or teeth or mouth or periodont* or gingival* or "oral health")) AND CENTRAL:TARGET

19 . $\# 1$ or \#2 or \#3 or \#4 or \#5 or \#6 or \#7 or \#8 or \#9 or \#10 or \#11 or \#12 or \#13 or \#14 or \#15 or \#16 or \#17 or \#18

20.MESH DESCRIPTOR Intellectual disability EXPLODE ALL AND CENTRAL:TARGET

21.MESH DESCRIPTOR Developmental disability AND CENTRAL:TARGET

22.((intellectual or mental) and "developmental disorder") AND CENTRAL:TARGET

23. ((intellectual* ${ }^{\star}$ or mental ${ }^{\star}$ or learning) NEAR5 (disabl ${ }^{\star}$ or disabilit* or deficien ${ }^{\star}$ or impair $^{\star}$ or handicap $\left.{ }^{\star}\right)$ AND CENTRAL:TARGET

24. ((deficien* or low $\left.{ }^{\star}\right)$ NEAR3 (cognition or "cognitive function*" or reason* or intelligence)) AND CENTRAL:TARGET

25.("special needs" or (special NEAR3 child\$) or retard* or "slow learner*") AND CENTRAL:TARGET

26.("Down* syndrome" or mongol* or "De Lange syndrome" or "Prader Willi syndrome" or "Labhart Willi syndrome" or "Royer syndrome" or "Rubinstein-Taybe syndrome" or "Rubinstein syndrome" or "WAGR syndrome" or "Williams syndrome" or "Broad Thumb Hallux syndrome") AND CENTRAL:TARGET

$27 . \# 20$ or \#21 or \#22 or \#23 or \#24 or \#25 or \#26

28.\#19 and \#27 


\section{MEDLINE Ovid search strategy}

1. Dental care for disabled/

2. exp Oral hygiene/

3. Oral health/

4. exp Periodontal diseases/

5. exp Periodontics/

6. (periodont\$ or gingiv\$).ti,ab.

7. exp Dental health surveys/

8. (toothbrush\$ or tooth-brush\$ or floss\$ or "chewing stick\$" or "wood stick\$" or toothpick\$).ti,ab.

9. (caries or carious).ti,ab.

10. exp dental caries/

11. ((dental or oral or mouth or interdental or interproximal or tooth or teeth or orthodontic or denture\$ or brace $\$$ or bracket\$) adj3 (irrigat $\$$ or clean\$ or brush\$ or clens\$ or aid\$)).ti,ab.

12. ((oral or dental) adj2 (hygiene or care)).ti,ab.

13. ((mouth or teeth) adj3 care).ti,ab.

14. (plaque\$ adj5 (remov\$ or control\$)).ti,ab.

15. ("dental plaque index" or "dental plaque indices" or "DMF\$ index" or "DMF indices" or "dmf\$ index" or "dmf\$ indices" or "periodontal index" or "periodontal indices" or "oral hygiene index" or "oral hygiene indices" or "gingival index").ti,ab.

16. Dental plaque/

17. Health education, dental/

18. ((health\$ adj3 promot\$) and (dental or teeth or mouth or periodont\$ or gingival\$ or "oral health")).ti,ab.

19. or/1-18

20. exp Intellectual disability/

21. Developmental disabilities/

22. ((intellectual or mental) and "developmental disorder").ti,ab.

23. ((intellectual\$ or mental\$ or learning) adj5 (disabl\$ or disabilit\$ or deficien\$ or impair\$ or handicap\$)).ti,ab.

24. ((deficien\$ or low\$) adj3 (cognition or "cognitive function\$" or reason\$ or intelligence)).ti,ab.

25. ("special needs" or (special adj3 child\$) or retard\$ or "slow learner\$").ti,ab.

26. ("Down\$ syndrome" or mongol\$ or "De Lange syndrome" or "Prader Willi syndrome" or "Labhart Willi syndrome" or "Royer syndrome" or "Rubinstein-Taybe syndrome" or "Rubinstein syndrome" or "WAGR syndrome" or "Williams syndrome" or "Broad Thumb Hallux syndrome").ti,ab.

27. or/20-26

28. 19 and 27

\section{Embase Ovid search strategy}

1. Mouth hygiene/

2. exp Periodontal disease/

3. exp Periodontics/

4. (periodont\$ or gingiv\$).ti,ab.

5. (toothbrush\$ or tooth-brush\$ or floss\$ or "chewing stick\$" or "wood stick\$" or toothpick\$).ti,ab. 
6. (caries or carious).ti,ab.

7. exp dental caries/

8. ((dental or oral or mouth or interdental or interproximal or tooth or teeth or orthodontic or denture\$ or brace\$ or bracket\$) adj3 (irrigat

\$ or clean\$ or brush\$ or clens\$)).ti,ab.

9. (dentifrice\$ or mouthwash\$ or mouthrins\$ or mouth-wash\$ or mouth- rins\$).ti,ab.

10. ((oral or dental) adj2 (hygiene or care)).ti,ab.

11. ((mouth or teeth) adj3 care).ti,ab.

12. (plaque\$ adj5 (remov\$ or control\$)).ti,ab.

13. ("dental plaque index" or "dental plaque indices" or "DMF\$ index" or "DMF indices" or "dmf\$ index" or "dmf\$ indices" or "periodontal index" or "periodontal indices" or "oral hygiene index" or "oral hygiene indices" or "gingival index").ti,ab.

14. Tooth plaque/

15. Dental health education/

16. ((health\$ adj3 promot\$) and (dental or teeth or mouth or periodont\$ or gingival\$ or "oral health")).ti,ab.

17. or/1-16

18. exp Intellectual impairment/

19. Developmental disorder/

20. ((intellectual or mental) and "developmental disorder").ti,ab.

21. ((intellectual\$ or mental\$) adj5 (disabl\$ or disabilit\$ or deficien\$ or impair\$ or handicap\$)).ti,ab.

22. ((deficien\$ or low\$) adj3 (cognition or "cognitive function\$" or reason\$ or intelligence)).ti,ab.

23. ("special needs" or (special adj3 child\$) or retard\$).ti,ab.

24. ("Down\$ syndrome" or mongol\$ or "De Lange syndrome" or "Prader Willi syndrome" or "Labhart Willi syndrome" or "Royer syndrome" or "Rubinstein- Taybe syndrome" or "Rubinstein syndrome" or "WAGR syndrome" or "Williams syndrome" or "Broad Thumb Hallux syndrome").ti,ab.

25. or/18-24

26. 17 and 25

\section{PsycINFO Ovid search strategy}

1. Oral health/

2. (periodont\$ or gingiv\$).ti,ab.

3. (toothbrush\$ or tooth-brush\$ or floss\$ or "chewing stick\$" or "wood stick\$" or toothpick\$).ti,ab.

4. (caries or carious).ti,ab.

5. ((dental or oral or mouth or interdental or interproximal or tooth or teeth or orthodontic or denture\$ or brace\$ or bracket\$) adj3 (irrigat

\$ or clean\$ or brush\$ or clens\$)).ti,ab.

6. (dentifrice\$ or mouthwash\$ or mouthrins\$ or mouth-wash\$ or mouth- rins\$).ti,ab.

7. ((oral or dental) adj2 (hygiene or care)).ti,ab.

8. ((mouth or teeth) adj3 care).ti,ab.

9. (plaque\$ adj5 (remov\$ or control\$)).ti,ab

10. ("dental plaque index" or "dental plaque indices" or "DMF\$ index" or "DMF indices" or "dmf\$ index" or "dmf\$ indices" or "periodontal index" or "periodontal indices" or "oral hygiene index" or "oral hygiene indices" or "gingival index").ti,ab.

11. ((health\$ adj3 promot\$) and (dental or teeth or mouth or periodont\$ or gingival\$ or "oral health")).ti,ab.

12. or/1-11

13. exp Intellectual development disorder/

14. exp Developmental disabilities/

15. exp Learning disorders/

16. ((intellectual or mental) and "developmental disorder").ti,ab.

17. ((intellectual\$ or mental\$) adj5 (disabl\$ or disabilit\$ or deficien\$ or impair\$ or handicap\$)).ti,ab.

18. ((deficien\$ or low\$) adj3 (cognition or "cognitive function\$" or reason\$ or intelligence)).ti,ab.

19. ("special needs" or (special adj3 child\$) or retard\$).ti,ab.

20. ("Down\$ syndrome" or mongol\$ or "De Lange syndrome" or "Prader Willi syndrome" or "Labhart Willi syndrome" or "Royer syndrome" or "Rubinstein- Taybe syndrome" or "Rubinstein syndrome" or "WAGR syndrome" or "Williams syndrome" or "Broad Thumb Hallux syndrome").ti,ab.

21. or $/ 13-20$

22. 12 and 21

\section{US National Institutes of Health Ongoing Trials Register (ClinicalTrials.gov) search strategy}

intellectual and "oral health"

intellectual and dental

"developmental disorder" and "oral health"

"developmental disorder" and dental 


\section{World Health Organization International Clinical Trials Registry Platform search strategy}

intellectual and "oral health"

intellectual and dental

"developmental disorder" and "oral health"

"developmental disorder" and dental

"special needs" and "oral health"

"special needs" and dental

\section{Appendix 2. Characteristics of studies template}

\begin{tabular}{ll}
\hline Methods & Study design \\
Date of study \\
Study duration \\
Setting \\
Ethical approval \\
Consent
\end{tabular}

Participants
Conversion to ICD description
Age range (mean)
Gender
Comorbidity reported/details
Number of participants at baseline
Number of participants at final evaluation
Selection of the participants for the intervention
Country

Intervention

\section{Comparison}

Group 1

Group 2 etc

Outcomes Outcomes measured

1.

2.

Timing of outcome assessments

\section{COM-B system characteristics Potential sources of behaviour change}

Potential intervention functions

\section{Stakeholder involvement Formal or non-formal carer}

With or without dental professional involvement 


\begin{tabular}{lll}
\hline Date & Event & Description \\
\hline 3 June 2019 & Amended & Search date edit in Plain Language Summary \\
\hline
\end{tabular}

\section{CONTRIBUTIONS OFAUTHORS}

Catherine Waldron: conceived and co-ordinated the review, assisted in developing the search strategies, organised the retrieval of the papers and unpublished data, contacted authors of the papers for further information and entered the data in Review Manager 2014. The protocol and the review were written by her with the input of the expert knowledge of the other team members and the Cochrane Oral Health editorial team. The screening and extraction of data was carried out by her and four other team members. The data analysis was carried out by her with the input of the team statisticians. The interpretation of the data was agreed by the whole team. She approved the final version of the review.

June Nunn: provided expert knowledge on oral health and intellectual disability during the writing of the protocol and review. She was involved in the screening and extraction of the data and commented on the data analysis, commented on and agreed the interpretation of the data, and approved the final version of the review.

Caoimhin Mac Giolla Phadraig: provided expert knowledge on oral health and intellectual disability during the writing of the protocol and review. He was involved in the screening and extraction of the data and commented on the data analysis, commented on and agreed the interpretation of the data and approved the final version of the review.

Catherine Comiskey: provided expert knowledge on research methodology and statistical analysis during the writing of the protocol and review. She was involved in the screening and extraction of the data and commented on the data analysis, agreed the interpretation of the data and approved the final version of the review.

Suzanne Guerin: provided expert knowledge on psychological interventions in research and clinical settings and on disability in general. She was involved in the screening and extraction of the data and commented on the data analysis, agreed the interpretation of the data and approved the final version of the review.

Maria Theresa van Harten: provided detailed expert knowledge on the statistical analysis during the writing of the review and the interpretation of the data, agreed the interpretation of the data and approved the final version of the review.

Erica Donnelly-Swift: provided expert knowledge on the statistical analysis planned for the review during the writing of the protocol and the review and approved the final version of the review.

Mike J Clarke: provided expert knowledge on Cochrane Review methodology, commented on the drafts of the protocol and the review, commented on the data analysis, agreed the interpretation of the data and approved the final version of the review.

\section{DECLARATIONS OF INTEREST}

Catherine Waldron: I have been sponsored by Wrigley's Ireland (current) and the Sunstar Foundation (complete) to design and present oral health promotional activities. The content of these presentations and activities has not been in any way influenced by the sponsors. I received a Health Research Board Cochrane Training Fellowship, which supported the development of this review.

June Nunn: I was a member of a consensus working group in 2015, sponsored by Johnson and Johnson, to develop a position statement on the management of periodontal disease in the community, for submission to the Department of Health's Oral Health Strategy Review Group 
in Ireland. I withdrew as a signatory to the final document as I was not prepared to endorse the use of designated commercial mouthwashes in the situation where routine oral hygiene measures were not possible. I receive small sums as royalties on textbooks and chapters I have published on dental topics. I was co-author on an included RCT, but other review authors dealt with its inclusion in this review.

Caoimhin Mac Giolla Phadraig: I have no conflicting interests that would influence my actions on this Cochrane Review. For completeness, I report that I was recipient of a joint Irish Dental Association and Wrigley Foundation award for oral health promotion in 2018 and 2019 , which supported the development of toothbrushing resources online. I was lead author on an included RCT, but other review authors dealt with its selection for this review.

Catherine Comiskey: I have no conflicts that would have any influence on my impartiality within the current review. For completeness, I report that I have undertaken programme evaluations through my university (Blossom Ireland programmes, Childhood Development Intiative Healthy School Program, AAL Call 7 Breath Project); I am chairperson of the NACDA (National Advisory Committee on Drugs and Alcohol); and I am a consultant for SANTRUST, which delivers PhD education in South Africa.

Suzanne Guerin: I have no conflicting interests that would affect my participation in this review, but for completeness, I report that I am an unpaid Board Member for the Tallaght West Childhood Development Initiative; I held a grant from Atlantic Philantropies for a study of respite care in intellectual disabilities; and I currently hold an H2020 grant on a topic unrelated to this review. I was co-author on an included RCT, but other review authors dealt with its inclusion in this review. I was co-author on an included RCT, but other review authors dealt with its inclusion in this review.

Maria Theresa van Harten: none.

Erica Donnelly-Swift: none.

Mike J Clarke: none. I have a variety of roles within Cochrane including serving as Co-ordinating Editor of the Methodology Review Group.

\section{SOURCES OF SUPPORT}

\section{Internal sources}

- Dublin Dental University Hospital, Trinity College, Dublin 2, Ireland.

\section{External sources}

- National Institute for Health Research (NIHR), UK.

This project was supported by the NIHR, via Cochrane Infrastructure funding to Cochrane Oral Health. The views and opinions expressed therein are those of the authors and do not necessarily reflect those of the Systematic Reviews Programme, NIHR, National Health Service (NHS) or the Department of Health.

- Cochrane Oral Health Global Alliance, Other.

The production of Cochrane Oral Health reviews has been supported financially by our Global Alliance since 2011 (ohg.cochrane.org/ partnerships-alliances). Contributors over the past year have been the American Association of Public Health Dentistry, USA; ASAkademie, Germany; British Association for the Study of Community Dentistry, UK; the British Society of Paediatric Dentistry, UK; the Canadian Dental Hygienists Association, Canada; the Centre for Dental Education and Research at All India Institute of Medical Sciences, India; the National Center for Dental Hygiene Research \& Practice, USA; New York University College of Dentistry, USA; and Swiss Society for Endodontology, Switzerland

\section{DIFFERENCES BETWEEN PROTOCOLANDREVIEW}

Following a pilot of a small number of studies of the full-text reading process by the review team, we made some amendments and clarifications.

1. In relation to the definition of oral hygiene, we made the following clarification.

a. Interventions focusing on the chemical removal of plaque alone were excluded. The use of a chemical agent, which is used on a toothbrush and arguably removes plaque both mechanically and chemically, is complex, as the measurement of the resulting reduction of plaque cannot be assigned to either solely the mechanical or chemical action. These studies were excluded, except for those studies using a conventional non-prescription toothpaste normally used by the participants or using a non-conventional chemical agent for both the intervention and control groups in studies comparing the mechanical removal of plaque. For example, a study comparing a manual toothbrush with an electric toothbrush where both were used with a non-conventional toothpaste such as chlorohexidine or extra-strength fluoride toothpaste. 
2. In relation to the study designs we made the following clarifications:

a. The study designs to be included in this review used the EPOC terminology and definitions except for quasi-randomised trials, which used the Cochrane glossary definition (Cochrane 2018; EPOC 2017).

b. The study designs included quasi-randomised trials.

c. Cluster-randomised trials, non-random controlled trials and controlled before-after studies were included even if there was only one intervention site and one control site, as long as the underlying differences between the sites would not overwhelm the true difference between the intervention and the control. For example, if an intervention was used for people living in a residential care home setting while the control was with people who were living in their own homes, the populations might be too different to begin with. These types of study designs with only one intervention and one control site are commonly used in interventions for populations with ID where randomised controlled trials can be more difficult to implement. Therefore, to make this review relevant, it was important to include these studies so that the effectiveness of these types of interventions could be analysed.

d. Quasi-randomised trials were defined as; methods of allocating people to a trial that were not random but were intended to produce similar groups when used to allocate participants. Quasi-random methods included: allocation by the person's date of birth, by the day of the week or month of the year, by a person's medical record number or just allocating every alternate person. In practice, these methods of allocation are relatively easy to manipulate, introducing selection bias.

e. Interrupted time series, where multiple data points were collected before and after the intervention and the intervention effect was measured against the preintervention trend and repeated measure studies, where these measures were taken on the same people at each time point, were only included if they had a clearly defined point in time when the intervention occurred and at least three data points before and three after the intervention.

3. The descriptions of the some of the secondary outcomes were amended to more clearly describe what was being measured: skills and adherence to routines were combined and renamed as 'behaviour, attitude and self-efficacy.'

4. Of the two tools identified for possible use in relation to the assessment of risk of bias in the non-controlled trials, we opted to use the more practical Effective Practice and Organisation of Care (EPOC) criteria for assessing risk of bias (EPOC 2016; Sterne 2016), rather than the more cumbersome Risk Of Bias In Non-randomised Studies - of Interventions (ROBINS-I) assessment tool.

\section{INDEX TERMS}

\section{Medical Subject Headings (MeSH)}

${ }^{\star}$ Intellectual Disability; ${ }^{\star}$ Oral Health; ${ }^{*}$ Oral Hygiene; Dental Plaque; Periodontal Diseases [ ${ }^{\star}$ prevention \& control]; Toothbrushing [methods]

\section{MeSH check words}

Humans 